\title{
Second Spectrum of Tungsten (W II)
}

\author{
Donald D. Laun ${ }^{1}$
}

(October 30, 1963)

\begin{abstract}
A preliminary report on the second spectrum of tungsten, published in 1938, presented 27 even energy levels and 50 odd energy levels that were derived from $500 \mathrm{~W}$ II lines ranging in wavelength from $1961.43 \AA$ to $4348.13 \AA$. The present paper submits data on 62 even levels, 132 odd levels, and 2,173 classified lines of W II, ranging in wavelength from $1756.6 \AA$ to $6219.77 \AA$. The ground state of the $\mathrm{W}^{+}$ion is represented by the ${ }^{6} \mathrm{D}_{01 / 2}$ level of a sextet $\mathrm{D}$ term arising from the $5 d^{4} 6 s^{1}$ electron configuration, but the level intervals and magnetic splitting factors indicate considerable departure from $L S$-coupling, suggesting that coupling intermediate between $L S$ and $J j$ may be more appropriate for the spectrum W II.
\end{abstract}

\section{Introduction}

The search for regularities among spectral lines emitted by tungsten arcs and sparks was initiated at the National Bureau of Standards in 1925 when O. Laporte and I were both new (temporary) members of the Spectroscopy Section. By exploiting existing data on wavelengths, intensities, and Zeeman patterns, Laporte $[1]^{2}$ identified the first spectral terms and classified lines of $W_{I}$. Zeeman data confirmed the designation of the six lowest energy levels as ${ }^{5} \mathrm{D}$ from $5 d^{4} 6 s^{2}$ and ${ }^{7} \mathrm{~S}$ from $5 d^{5} 6 s^{1}$. This term analysis and quantum interpretation of $W_{\text {I }}$ was later greatly extended by Laporte and Mack [2] until it included 300 energy levels and 2,378 classified lines ranging in wavelength from $2008.64 \AA$ to $11477.97 \AA$.

Shortly after Laporte found the low-energy terms in W I, I began a search for the theoretical low ${ }^{6} \mathrm{D}$ (from $5 d^{4} 6 s^{1}$ ) and ${ }^{6} \mathrm{~S}$ (from $5 d^{5}$ ) terms in $\mathrm{W}_{\mathrm{II}}$, assuming that ionization removed one $s$ electron. This initial search failed because of insufficient information about the spectrum W II. Then I undertook to reobserve the ultraviolet arc and spark spectra of tungsten, to distinguish W II from W I, to refine the wavelengths, and to observe Zeeman patterns of $\mathrm{W}$ II lines. From these observations, 27 even levels (including ${ }^{6} \mathrm{D}$ and ${ }^{6} \mathrm{~S}$ ) and 50 odd levels were derived from $500 \mathrm{~W}$ ir lines ranging in wavelength from $1961.43 \AA$ to $4348.13 \AA$; these results were published [3] in 1938 as a preliminary report on lines and levels of the spectrum $\mathrm{W}$ iI. From time to time during the following quarter century, further improvements in observations and extensions of analyses have been made; the purpose of the present paper is to present the final data on 62 even levels, 132 odd levels, and 2,173 classified lines of $\mathrm{W}_{\mathrm{II}}$, ranging in wavelength from $1756.6 \AA$ to $6219.77 \AA$.

${ }^{1}$ Former member of Spectroscopy Section, NBS; present address: Kiel, Wis. ${ }_{2}$ Figures in brackets indicate the literature references at the end of this paper.

\section{Experimental Procedure}

Small rods of very pure tungsten were used as electrodes in the arcs and sparks that served as light sources. The arcs were operated with applied potential of $220 \mathrm{v}$ and 6 amp de, the sparks with $30,000 \mathrm{v}$, about $30 \mathrm{ma}$, ac, and $0.006 \mu$ f capacitance.

The first spectrograms were made in 1925 with a Hilger E 1 (Littrow) quartz spectrograph which could be effectively employed in the ultraviolet down to wavelength 1900 A. Later, these spectrograms were supplemented by a new series obtained by C. C. Kiess with the first Hilger E 185 (Littrow) quartz spectrograph that was delivered to the Bureau of Standards in 1932. This larger spectrograph has a focal length of $3 \mathrm{~m}$, and reciprocal dispersion, or plate factor, of $0.3 \AA / \mathrm{mm}$ at $2100 \AA$ and $1 \AA / \mathrm{mm}$ at 3000 A. These observations of tungsten lines on photographic plates exposed in quartz spectrographs were presented by me as a Master's 'Thesis at the University of Chicago [4]. Additional spectrograms were made in 1933 by Kiess with a concave grating of $22-\mathrm{ft}$ radius, and 30,000 lines per inch. In a Wadsworth mounting, this grating produced spectra down to $1900 \AA$ with a plate factor of $2.44 \AA / \mathrm{mm}$ in the first order, and with a plate factor of $0.88 \AA / \mathrm{mm}$ in the second order from 3200 to $4400 \AA$. This grating spectrograph was employed also to photograph tungsten spectra throughout the visible range.

Since all the above-mentioned spectrographs were stigmatic, movable diaphragms before the slits permitted placing strips of juxtaposed spectra on photographic plates. The usual procedure was to photograph the iron-are spectrum containing standard wavelengths, then the tungsten ares and sparks in adjacent strips and another iron spectrum beside the tungsten spark. The positions of tungsten spectral lines relative to iron lines were measured on Gaertner comparators, in 1925-26 at the Bureau of Standards; and in 1933-34 at Marquette University by courtesy of the late Fr. Joseph Carroll, S. J. Linear scale readings allowed the wavelengths of tungsten lines to be interpolated between iron 
standards in the case of prismatic spectra with the aid of Hartmann's dispersion formula, and from grating spectrograms by assuming linear dispersion. In both cases, small corrections were made when the interpolated values of iron wavelengths departed from their standard values. For purposes of analysis, the final wavelengths of tungsten lines were converted to vacuum wavenumbers with the aid of the Table of Wavenumbers published by Coleman, Bozman, and Meggers [5].

At the same time that the positions or wavelengths of tungsten lines were being measured, their relative intensities were estimated in both sources. Although this intensity scale, ranging from 1 for a barely discernible line to 1000 for the strongest, is more or less arbitrary, it is primarily useful in separating W II from W $\mathrm{I}$, and also qualitatively supports the subsequent analysis in which intensities are related to quantum numbers. In some cases W II lines appear to coincide with $\mathrm{W}_{\mathrm{I}}$, and if a $\mathrm{W}_{\mathrm{II}}$ line sometimes appears stronger in the arc than in the spark, it may be an example of pole effect. Unfortunately, no deliberate attempt to exclude or exploit pole effect in the tungsten arc was made.

Finally, some information about tungsten spectra in the vacuum ultraviolet was obtained by measuring two spectrograms made by J. C. Boyce at the Massachusetts Institute of Technology in 1940 and kindly presented to the Bureau of Standards. These spectrograms were obtained with an evacuated grating spectrograph having a plate factor of $4.26 \AA / \mathrm{mm}$. The spectra were measured down to $1200 \AA$. Although the excitation conditions were altered, the differences between spectra were so slight that only above $1756 \AA$ was it possible positively to identify $\mathrm{W}$ II lines. The wavelengths from this point to $1950 \AA$ were finally corrected to the scale of values calculated from atomic energy levels.

My preliminary paper [3] of 1938 reported Zeeman-effect measurements of spectrograms made with a Weiss magnet and quartz spectrographs at the Bureau of Standards. These data have been superseded by those obtained with the more powerful Bitter magnet and grating spectrographs at the Massachusetts Institute of Technology. These results will be mentioned in the next section.

\section{Results}

The main result of the experiments mentioned above is that the wavelengths of about 13,000 spectral lines characteristic of ares and sparks between tungsten electrodes have been measured. Among these, about 4,000 have been attributed to $\mathrm{W}$ II and 2,173 of these lines, including 90 percent of the total intensity, have been shown to arise from transitions between 194 derived energy levels, 62 even and 132 odd. The even levels are displayed in table 1 and the odd levels in table 2 . In these tables, the electron configuration is given in col- umn 1, the spectral term designation in column 2, total angular momentum, $J$, in column 3 , relative values of energy levels in column 4, intervals between levels of complex terms in column 5, and observed magnetic splitting factor, $g$, in column 6 . Most of these data were supplied in 1956 for the third volume of Atomic Energy Levels [6] which contains $J$ and level values for 45 even and 90 odd levels. In that volume, $g$-factors were reported for 38 even levels and for 58 odd. These were derived from MIT Zeeman spectrograms measured by J. E. Mack and Mrs. Taschek at the University of Wisconsin. Since 1956, the number of even levels has increased from 45 to 62 and odd levels from 90 to 132 . Likewise, $g$-factors are now available for 46 even and 71 odd levels as compared with 38 and 58 respectively, in 1956.

In Atomic Energy Levels [6], it is stated that because of departure from $L S$-coupling it is difficult to assign term designations except for the lowest terms. Consequently, only six even terms, comprising 21 levels between 0 and $17437 \mathrm{~K}$, were completely grouped and designated, and 24 higher even levels, between 18000 and $26929 \mathrm{~K}$, were tentatively designated, guided by analogy with Mo II, $g$-factors, and theoretical calculations of the late R. E. Trees. Likewise, two odd terms, comprising six levels, were tentatively designated ${ }^{6} \mathrm{~F}$ and ${ }^{2} \mathrm{~S}$, but the remaining 84 levels were given as miscellaneous. In the present paper, additional levels and $g$-factors are reported but no revision or extension of designations has been made.

The spectral lines of tungsten from which all the information in tables 1 and 2 was derived, are listed in table 3 in order of increasing wavelength. Each line is described by its measured wavelength in angstroms, by its estimated intensity in a tungsten are and/or spark, by its appropriate vacuum wavenumber in kaysers $\left(1 \mathrm{~K}=1 \mathrm{~cm}^{-1}\right)$, by the difference $(O-C)$ between the observed wavenumber and that calculated from the combination of energy levels shown in the next-to-last column. Abbreviated notes about the observed Zeeman patterns for 307 lines appear in the final column.

Table 3 contains 2,173 classified lines of $\mathrm{W}$ II, including 42 doubly classified. The only other table of classified W II lines was published [3] in 1938; it contained 500 lines, including 6 doubly classified. In addition to 2,173 classified lines, table 3 also contains wavelengths, intensities, and wavenumbers of 25 of the strongest W II lines still unclassified; these are included with the hope that the blank spaces under "combinations" may eventually be filled in.

The "Intensity" column of table 3 shows that the great majority of these $W_{\text {II }}$ lines were observed in arc as well as spark sources but with generally higher intensity in the latter when spectrogram exposures were chosen to make $W_{I}$ lines appear in both sources with nearly equal intensities. In a few cases, $W_{\text {II }}$ lines appear only, or stronger, in arc spectra; most if not all such deviations may be explained by coincident $W_{\text {I lines, pole effects, or air lines that occa- }}$ 
sionally mask metal lines in sparks operated at atmospheric pressure. Where the letter $A$ follows an arc intensity, a line with nearly identical wavelength has actually been classified as a combination of W I energy levels. The letter $d$ in table 3 indicates a double line.

The observed minus calculated wavenumbers $(O-C)$ have an average value of $0.1 \mathrm{~K}$ for wavelengths measured in air, that is, above $2000 \AA$. This means that the average error in these wavelength measurements is of the order of $0.01 \AA$, and there is high confidence that the great majority of the combinations are real, rather than accidental. However, in the vacuum ultraviolet, below $2000 \AA$, the average $O-C$ is considerably greater, in a few cases as large as $5 \mathrm{~K}$, which entails an error in wavelength of the order of $0.2 \AA$. In such cases, confidence that the combinations are genuine is greatly reduced but they have been tentatively retained for the following reasons. Since the scale of wavenumbers is greatly compressed in the vacuum region, and the tungsten spectra below $2000 \AA$ were photographed with relatively small dispersion and measured without adequate standard wavelengths, it was inevitable that these measured wavelengths of $\mathrm{W}_{\text {II }}$ lines would be more or less uncertain. Furthermore, it appears that most of these $W_{I I}$ short waves involve the lowest and adjacent metastable energy levels, which is precisely what should be expected of strong lines with high energy. Therefore, these combinations with relatively large $O-C$ may be regarded as real pending wavelength measurements of higher accuracy.

In the introduction, I mentioned that the first spectral terms, both in $W_{I}$ and in $W_{I I}$, were found from Zeeman-effect observations. In both spectra, such observations have greatly facilitated their analyses, and constitute the supreme test of validity. In the last column of table 3 , are found some notes about the observed Zeeman patterns of 307 W lines extending from $2216 \AA$ to $4358 \AA$. The Zeeman patterns of $W_{\text {I }}$ and $W_{\text {II }}$ on MIT spectrograms were measured at the University of Wisconsin by J. E. Mack and Mrs. Taschek who supplied most of the magnetic splitting factors ( $g$-factors) for tungsten levels that appear in Atomic Energy Levels [6]. Recently, those spectrograms were kindly loaned to me for examination. Without giving any details, I have merely written "res" to indicate resolved Zeeman patterns that agree with the combinations of $W_{\text {II }}$ lines in table 3 . Unresolved Zeeman patterns are numbered $4,5,6$, and 7 , according to the notation of Back and Landé [7]. Incidentally, Zeeman types 4,5 , and 6 uniquely characterize, and fix $J$-values of, energy levels belonging to even multiplicities such as those responsible for $W_{\text {II }}$ lines. All the Zeemaneffect spectrograms are thickly covered by patterns

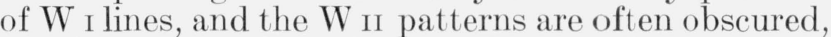
or vice versa, but despite these difficulties, the combinations are generally verified.

Finally, reference is made to the Tables of SpectralLine Intensities by Meggers, Corliss, and Scribner [8] which list 1,300 tungsten lines, $1168 \mathrm{~W}$ I and $132 \mathrm{~W}$ II, observed in an are between copper electrodes containing 0.1 atomic percent of tungsten. It is noteworthy that no $\mathrm{W}_{\text {II }}$ lines appear among the first 28 lines of highest intensity; they are all $\mathrm{W}_{\mathrm{I}}$. Tungsten is similar to rhenium in this respect, but unlike thorium or uranium in which spark lines predominate in this type of arc source. Ionization potentials may be responsible for this difference, since the I.P. of copper is less than that of tungsten or rhenium but greater than that of thorium or uranium. In the list of all observed lines of tungsten [8], all of the $W_{\text {II }}$ lines are now classified, and will be given in this paper.

In conclusion, let me say that since my association with the Bureau of Standards in 1925-26, when this investigation began, and again in 1943-44, when I collaborated on the uranium project [9], this work on $W_{\text {II }}$ has been pursued as a part-time occasional avocation with the goal of finding order in a complex spectrum.

TABLE 1. Even levels of W II

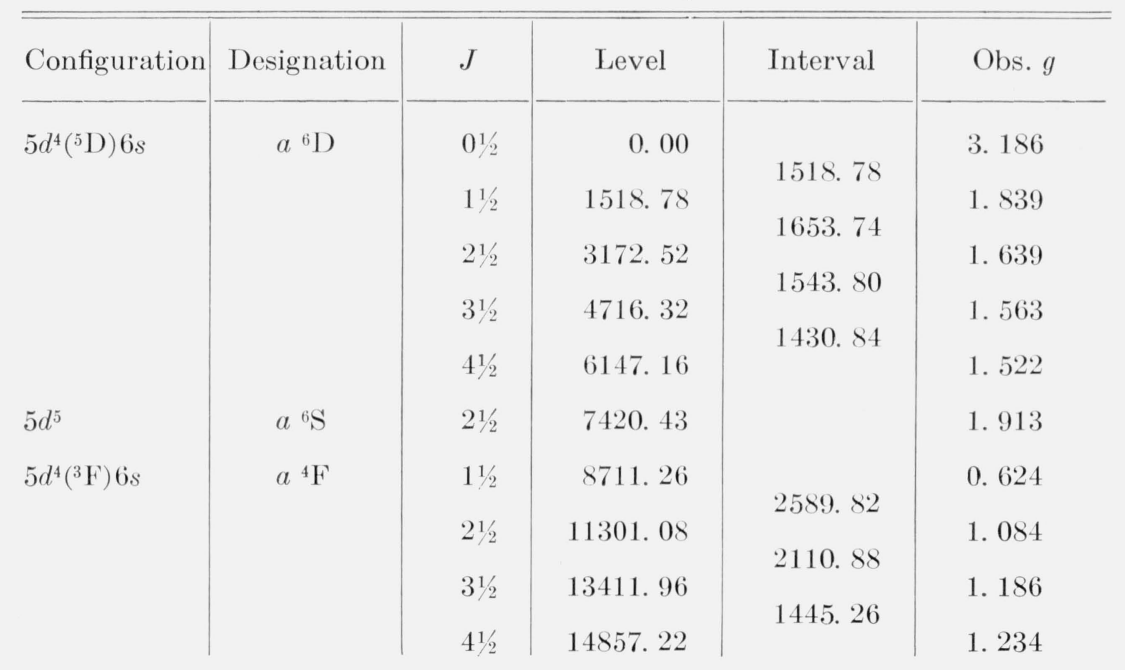


TABLE 1. Even levels of $W \mathrm{II}$-Continued

\begin{tabular}{|c|c|c|c|c|c|}
\hline Configuration & Designation & $J$ & Level & Interval & Obs. $g$ \\
\hline $5 d^{4}\left({ }^{3} \mathrm{P}\right) 6 s$ & $a{ }^{4} \mathrm{P}$ & $\begin{array}{l}01 / 2 \\
11 / 2 \\
21 / 2\end{array}$ & $\begin{array}{r}8832.66 \\
10592.52 \\
13434.10\end{array}$ & $\begin{array}{l}1759.86 \\
2841.58\end{array}$ & $\begin{array}{l}\text { 2. } 383 \\
\text { 1. } 471 \\
\text { 1. } 526\end{array}$ \\
\hline $5 d^{4}\left({ }^{5} \mathrm{D}\right) 6 s$ & $a{ }^{4} \mathrm{D}$ & $\begin{array}{l}01 / 2 \\
11 / 2 \\
21 / 2 \\
31 / 2\end{array}$ & $\begin{array}{l}13173.38 \\
14634.36 \\
14967.82 \\
15147.02\end{array}$ & $\begin{array}{r}1460.98 \\
333.46 \\
179.20\end{array}$ & $\begin{array}{l}0.455 \\
\text { 1. } 183 \\
\text { 1. } 013 \\
0.872\end{array}$ \\
\hline $5 d^{4}\left({ }^{3} \mathrm{G}\right) 6 s$ & $a^{4} \mathrm{G}$ & $\begin{array}{l}21 / 2 \\
31 / 2 \\
4^{1 / 2} \\
5^{1 / 2}\end{array}$ & $\begin{array}{l}16234.84 \\
16589.67 \\
16553.14 \\
17437.02\end{array}$ & $\begin{array}{r}354.83 \\
-36.53 \\
883.88\end{array}$ & $\begin{array}{l}0.995 \\
\text { 1. } 153 \\
\text { 1. } 137 \\
\text { 1. } 181\end{array}$ \\
\hline $\begin{array}{l}5 d^{4}\left({ }^{3} \mathrm{H}\right) 6 s \\
5 d^{5} \\
5 d^{5} \\
5 d^{5} \\
5 d^{4}\left({ }^{3} \mathrm{P}\right) 6 s \\
5 d^{4}\left({ }^{3} \mathrm{H}\right) 6 s \\
5 d^{5} \\
5 d^{5} \\
5 d^{5} \\
5 d^{5} \\
5 d^{4}\left({ }^{3} \mathrm{H}\right) 6 s \\
5 d^{5} \\
5 d^{5} \\
5 d^{4}\left({ }^{3} \mathrm{P}\right) 6 s \\
5 d^{4}\left({ }^{3} \mathrm{D}\right) 6 s \\
5 d^{5} \\
5 d^{5} \\
5 d^{5} \\
5 d^{5} \\
5 d^{5} \\
5 d^{5} \\
5 d^{4}\left({ }^{3} \mathrm{H}\right) 6 s \\
5 d^{5} \\
5 d^{5}\left({ }^{3} \mathrm{H}\right) 6 s\end{array}$ & $\begin{array}{l}{ }^{4} \mathrm{H} \\
{ }^{2} \mathrm{D} \\
{ }^{4} \mathrm{G} \\
{ }^{4} \mathrm{G} \\
{ }^{2} \mathrm{P} \\
{ }^{4} \mathrm{H} \\
{ }^{2} \mathrm{D} \\
{ }^{4} \mathrm{G} \\
{ }^{4} \mathrm{D} \\
{ }^{4} \mathrm{G} \\
{ }^{4} \mathrm{H} \\
{ }^{4} \mathrm{~F} \\
{ }^{4} \mathrm{~F} \\
{ }^{2} \mathrm{P} \\
\\
{ }^{4} \mathrm{D} \\
{ }^{4} \mathrm{~F} \\
{ }^{4} \mathrm{D} \\
{ }^{4} \mathrm{D} \\
{ }^{2} \mathrm{I} \\
\\
{ }^{4} \mathrm{~F} \\
\\
{ }^{4} \mathrm{P} \\
\\
{ }^{2} \mathrm{H} \\
{ }^{4} \mathrm{P} \\
{ }^{2} \mathrm{H}\end{array}$ & 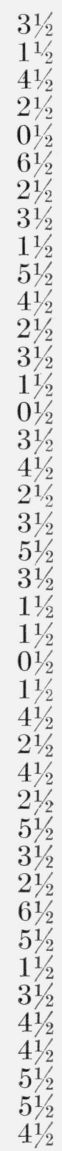 & $\begin{array}{l}18000.70 \\
18990.96 \\
19070.68 \\
19276.52 \\
19404.08 \\
19442.54 \\
19637.38 \\
20039.74 \\
20455.93 \\
20534.35 \\
20780.38 \\
22139.97 \\
22194.08 \\
22503.06 \\
22535.80 \\
23046.80 \\
23234.87 \\
23450.50 \\
23803.84 \\
23955.40 \\
24804.67 \\
24918.10 \\
24991.56 \\
25045.20 \\
25169.87 \\
25209.28 \\
25672.16 \\
26158.74 \\
26227.00 \\
26929.34 \\
27273.86 \\
28118.90 \\
28187.60 \\
28377.80 \\
28491.00 \\
28631.86 \\
29341.56 \\
30633.02 \\
31100.46 \\
33910.56 \\
34091.02\end{array}$ & & $\begin{array}{l}1.098 \\
0.90 \\
1.102 \\
0.997 \\
0.64 \\
\text { 1. } 102 \\
\text { 1. } 107 \\
0.51 \\
\text { 1. } 197 \\
1.065 \\
1.06 \\
1.119 \\
1.22 \\
2.2 \\
0.86 \\
1.249 \\
1.297 \\
\text { 1. } 10 \\
1.10 \\
0.9 \\
0.32 \\
1.64 \\
0.9 \\
\text { 1. } 04\end{array}$ \\
\hline
\end{tabular}


TABLE 2. Odd levels of $\mathrm{W}$ II

\begin{tabular}{|c|c|c|c|c|c|}
\hline Configuration & Designation & $J$ & Level & Interval & Obs. g \\
\hline $5 d^{4}\left({ }^{5} \mathrm{D}\right) 6 p$ & $z^{6} \mathrm{~F}^{\circ}$ & $\begin{array}{l}01 / 2 \\
11 / 2 \\
21 / 2 \\
31 / 2 \\
41 / 2 \\
51 / 2\end{array}$ & $\begin{array}{l}36165.35 \\
39129.41 \\
42049.45 \\
4487 \% .18 \\
46493.43 \\
51495.00\end{array}$ & $\begin{array}{l}2964.06 \\
2920.04 \\
2827.73 \\
1616.25 \\
5001.57\end{array}$ & $\begin{array}{l}0.678 \\
1.147 \\
1.292 \\
\text { 1. } 277 \\
\text { 1. } 311 \\
\text { 1. } 054\end{array}$ \\
\hline $5 d^{4}\left({ }^{3} \mathrm{P}\right) 6 p$ & $z^{2} \mathrm{~S}^{\circ}$ & $\begin{array}{l}01 / 2 \\
21 / 2 \\
11 / 2 \\
31 / 2 \\
21 / 2 \\
01 / 2 \\
41 / 2 \\
11 / 2 \\
01 / 2 \\
11 / 2 \\
31 / 2 \\
21 / 2 \\
01 / 2 \\
11 / 2 \\
21 / 2 \\
11 / 2 \\
21 / 2 \\
51 / 2 \\
312 \\
11 / 2 \\
31 / 2 \\
01 / 2 \\
41 / 2 \\
21 / 2 \\
21 / 2 \\
11 / 2 \\
41 / 2 \\
31 / 2 \\
11 / 2 \\
21 / 2 \\
31 / 2 \\
21 / 2 \\
31 / 2 \\
01 / 2 \\
41 / 2 \\
01 / 2 \\
11 / 2 \\
31 / 2 \\
21 / 2 \\
11 / 2 \\
31 / 2 \\
41 / 2 \\
11 / 2 \\
01 / 2 \\
21 / 2 \\
41 / 2 \\
11 / 2 \\
51 / 2 \\
21 / 2 \\
01 / 2 \\
31 / 2 \\
21 / 2 \\
0112 \\
51 / 2 \\
31 / 2 \\
21 / 2 \\
41 / 2 \\
11 / 2 \\
11 / 2 \\
51 / 2 \\
41 / 2 \\
61 / 2 \\
21 / 2 \\
1 / 2\end{array}$ & $\begin{array}{l}38576.32 \\
39936.81 \\
42298.20 \\
42390.27 \\
44354.82 \\
44455.18 \\
44758.10 \\
44911.63 \\
4545 \% .02 \\
45553.70 \\
46175.42 \\
46355.40 \\
46625.27 \\
47179.94 \\
47413.33 \\
47588.64 \\
48284.48 \\
48332.73 \\
48830.70 \\
48982.86 \\
49124.52 \\
49154.50 \\
49181.04 \\
49242.10 \\
50292.33 \\
50430.95 \\
50863.05 \\
51045.25 \\
51254.40 \\
51438.03 \\
51863.03 \\
52087.02 \\
52275.28 \\
52355.11 \\
52567.15 \\
52593.72 \\
52803.00 \\
52901.74 \\
53113.52 \\
53329.71 \\
53338.07 \\
53369.97 \\
53422.98 \\
53440.17 \\
54026.24 \\
54056.54 \\
54137.20 \\
54229.06 \\
54375.82 \\
54485.60 \\
54498.57 \\
54704.61 \\
54760.06 \\
54958.58 \\
55022.86 \\
55162.30 \\
55392.37 \\
55488.01 \\
56084.30 \\
56376.45 \\
56413.64 \\
56439.60 \\
56544.40\end{array}$ & & 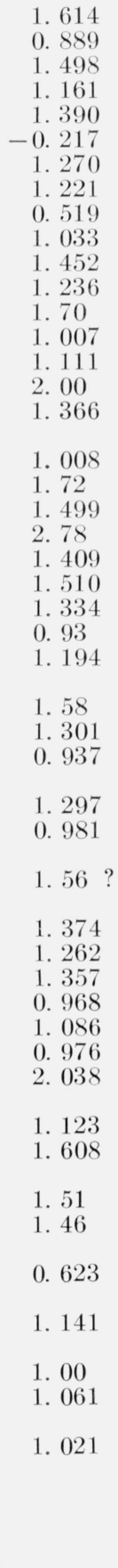 \\
\hline
\end{tabular}


TABLE 2. Odd levels of $\mathrm{W} \mathrm{II}$-Continued

\begin{tabular}{|c|c|c|c|c|c|}
\hline Configuration & Designation & $J$ & Level & Interval & Obs. g \\
\hline $5 d^{4}\left({ }^{3} \mathrm{P}\right) 6 p$ & $z^{2} \mathbf{S}^{0}$ & 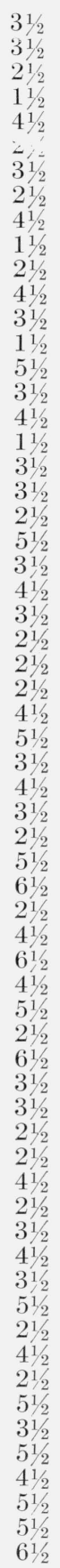 & $\begin{array}{l}56612.74 \\
56768.61 \\
56874.99 \\
56932.27 \\
57089.46 \\
57252.00 \\
57729.92 \\
57856.70 \\
57986.92 \\
58007.60 \\
58336.98 \\
58687.88 \\
58709.56 \\
58747.94 \\
58891.74 \\
59276.81 \\
59399.34 \\
59816.30 \\
59869.14 \\
59933.66 \\
59992.20 \\
60218.84 \\
60256.45 \\
60278.71 \\
60424.14 \\
6047.64 \\
60656.51 \\
60900.97 \\
61055.80 \\
61240.81 \\
61326.29 \\
61360.54 \\
61550.60 \\
61566.70 \\
61589.46 \\
61602.18 \\
62383.20 \\
62437.04 \\
62714.56 \\
62715.98 \\
62966.50 \\
62989.60 \\
63087.90 \\
63266.30 \\
63788.20 \\
63880.10 \\
64030.34 \\
64207.50 \\
64310.00 \\
64356.70 \\
64516.37 \\
64896.22 \\
64969.10 \\
64990.32 \\
65003.20 \\
65141.56 \\
65326.40 \\
65644.00 \\
65684.80 \\
66270.95 \\
66703.50 \\
68012.50 \\
68078.98\end{array}$ & & $\begin{array}{l}\text { 1. } 22 \\
\text { 1. } 147 \\
0.815 \\
\text { 1. } 06 \\
\text { 1. } 184 \\
\text { 1. } 36 \\
\text { 1. } 20 \\
\\
\text { 0. } 78 \\
\text { 1. } 144 \\
\text { 1. } 102 \\
\text { 1. } 179 \\
\text { 1. } 125 \\
\text { 1. } 130 \\
\\
\text { 1. } 07 \\
\text { 1. } 149\end{array}$ \\
\hline
\end{tabular}


Table 3. Classified lines of W II

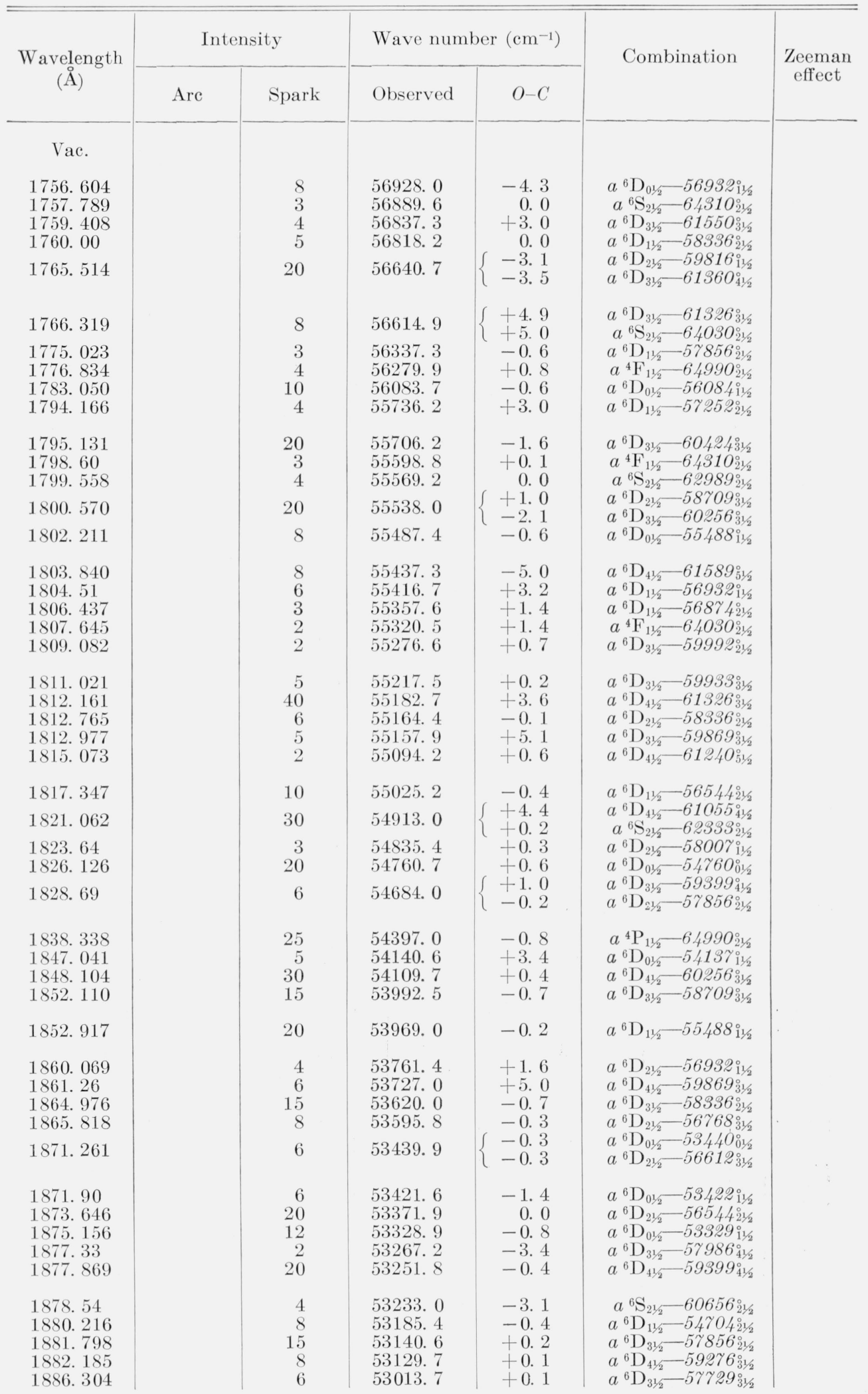


TABLE 3. Classified lines of $\mathrm{W}$ II-Continued

\begin{tabular}{|c|c|c|c|c|c|c|}
\hline \multirow{2}{*}{$\begin{array}{c}\text { Wavelength } \\
\text { (§) }\end{array}$} & \multicolumn{2}{|c|}{ Intensity } & \multicolumn{2}{|c|}{ Wave number $\left(\mathrm{cm}^{-1}\right)$} & \multirow{2}{*}{ Combination } & \multirow{2}{*}{$\begin{array}{c}\text { Zeeman } \\
\text { effect }\end{array}$} \\
\hline & Arc & Spark & Observed & $O-C$ & & \\
\hline \multicolumn{7}{|l|}{ Vac. } \\
\hline 1891.908 & & 6 & 52856.7 & $\left\{\begin{array}{l}-0.3 \\
+1.3\end{array}\right.$ & $\begin{array}{c}a^{6} \mathrm{D}_{11 / 2}-54375_{21 / 2}^{\circ} \\
a^{4} \mathrm{~F}_{11 / 2}-61566_{21 / 2}^{\circ}\end{array}$ & \\
\hline $\begin{array}{l}1892.442 \\
1893.80\end{array}$ & & $\begin{array}{r}15 \\
4\end{array}$ & $\begin{array}{l}52841.8 \\
52803.9\end{array}$ & $\begin{array}{r}+5.8 \\
+0.9\end{array}$ & $a{ }^{6} \mathrm{~S}_{21 / 2}-60256^{\circ}{ }^{\circ} 1 / 2$ & \\
\hline $\begin{array}{l}\text { 1893. } 80 \\
1895.943\end{array}$ & & 30 & $\begin{array}{l}52803.9 \\
52744.2\end{array}$ & $\begin{array}{l}+0.9 \\
-0.4\end{array}$ & $\begin{array}{l}a^{6} \mathrm{D}_{01 / 2}-52803_{11 / 2}^{\circ} \\
a^{6} \mathrm{D}_{41 / 2}-58891^{\circ 112}\end{array}$ & \\
\hline 1900.503 & & 12 & 52617.6 & -0.8 & $a^{6} \mathrm{D}_{11 / 2}-54137_{11 / 2}$ & \\
\hline 1901. 245 & & 30 & 52597.1 & +3.4 & $a^{6} \mathrm{D}_{01 / 2}-52593_{01 / 2}^{\circ}$ & \\
\hline 1902. 51 & & 20 & 52562.1 & -0.3 & $a^{6} \mathrm{D}_{41 / 2}-58709_{31 / 2}^{\circ}$ & \\
\hline $\begin{array}{l}1906.677 \\
1908.750\end{array}$ & & $\begin{array}{l}12 \\
10\end{array}$ & 52447.3 & -1.4 & $a^{6} \mathrm{~S}_{21 / 2}-59869_{31 / 2}^{\circ}$ & \\
\hline $\begin{array}{l}\text { 1908. } 750 \\
1909.16\end{array}$ & & 6 & $\begin{array}{l}52390.3 \\
52379.0\end{array}$ & $\begin{array}{l}-5.6 \\
+5.9\end{array}$ & $\begin{array}{c}a^{6} \mathrm{~S}_{21 / 2}-59816_{11 / 2}^{\circ} \\
a^{6} \mathrm{D}_{31 / 2}-57089^{\circ}{ }_{41 / 2}^{\circ}\end{array}$ & \\
\hline 1909. 80 & & 4 & 52361.4 & +6.3 & $a^{6} \mathrm{D}_{01 / 2}-52355_{01 / 2}^{\circ}$ & \\
\hline 1911.50 & & 25 & 52314.9 & -0.6 & $a^{6} \mathrm{D}_{21 / 2}-55488_{11 / 2}^{\circ}$ & \\
\hline 1915. 38 & & 8 & 52209.0 & -0.9 & $a^{4} \mathrm{P}_{21 / 2}-656443^{\circ} 1 / 2$ & \\
\hline $\begin{array}{l}1925.99 \\
1928.61\end{array}$ & & $\begin{array}{l}12 \\
20\end{array}$ & $\begin{array}{l}51921.3 \\
51850.8\end{array}$ & $\begin{array}{l}-0.1 \\
+0.5\end{array}$ & $a^{6} \mathrm{D}_{11 / 2}-53440_{01 / 2}^{\circ}$ & \\
\hline $\begin{array}{l}1928.61 \\
1929.47\end{array}$ & & 10 & $\begin{array}{l}51800.8 \\
51827.7\end{array}$ & $\begin{array}{l}+0.5 \\
-0.4\end{array}$ & $\begin{array}{l}a^{0} \mathrm{D}_{21 / 2}-550023_{31 / 2}^{1 / 2} \\
a^{6} \mathrm{D}_{31 / 2}-56544^{111 / 2}\end{array}$ & \\
\hline 1930. 10 & & 6 & 51810.8 & -0.1 & $a^{6} \mathrm{D}_{11 / 2}-53329_{11 / 2}^{\circ}$ & \\
\hline 1931.87 & & 10 & 51763.3 & -0.1 & $a^{4} \mathrm{~F}_{11 / 2}-6047421 \frac{1}{2}$ & \\
\hline 1934. 345 & & $\begin{array}{r}10 \\
3\end{array}$ & 51697.1 & -0.2 & $a^{6} \mathrm{D}_{31 / 2}-56413_{41 / 2}^{\circ}$ & \\
\hline $\begin{array}{l}1934.62 \\
1938.21\end{array}$ & & $\begin{array}{r}3 \\
25\end{array}$ & $\begin{array}{l}51689.7 \\
51594.0\end{array}$ & $\begin{array}{l}+1.2 \\
-0.7\end{array}$ & 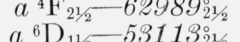 & \\
\hline & & & & & a $\mathrm{D}_{11 / 2}-00110_{21 / 2}$ & \\
\hline 1938.74 & & 3 & 51579.9 & $\begin{array}{l}-2.9 \\
+1.5\end{array}$ & $\begin{array}{l}a^{6} \mathrm{D}_{41 / 2}-57429^{\circ}{ }^{\circ} 12 \\
a^{4} \mathrm{~F}_{31}-64990^{\circ 11}\end{array}$ & \\
\hline 1940.54 & & 8 & 51532.0 & -0.1 & $a^{6} \mathrm{D}_{21 / 2}-547044_{21 / 2}^{10}$ & \\
\hline 1943. 27 & & 4 & 51459.6 & +0.2 & $a^{4} \mathrm{G}_{41 / 2}-68012_{51 / 2}^{\circ}$ & \\
\hline 1945. 02 & & $\begin{array}{l}10 \\
30\end{array}$ & 51413.5 & $\begin{array}{l}-0.2 \\
-0.5\end{array}$ & $a^{4} \mathrm{~F}_{41 / 2}-66270_{41 / 2}^{\circ}$ & \\
\hline 1948.35 & & 30 & 51325.5 & -0.5 & $a^{6} \mathrm{D}_{21 / 2}-54498_{31 / 2}^{\circ}$ & \\
\hline 1949.55 & & 40 & 51293.9 & +4.8 & $a^{6} \mathrm{~S}_{21 / 2}-58709_{31 / 2}^{\circ}$ & \\
\hline 1949. 93 & & $\begin{array}{r}4 \\
40\end{array}$ & 51283.9 & $\begin{array}{l}-0.3 \\
-0.2\end{array}$ & $a^{6} \mathrm{D}_{11 / 2}-52803_{11 / 2}^{\circ}$ & \\
\hline $\begin{array}{l}1951.06 \\
1953.00\end{array}$ & & $\begin{array}{r}40 \\
3\end{array}$ & $\begin{array}{l}51254.2 \\
51203.3\end{array}$ & $\begin{array}{r}-0.2 \\
0.0\end{array}$ & $\begin{array}{l}a^{6} \mathrm{D}_{01 / 2}-51254^{\circ 1} 12 \\
a^{6} \mathrm{D}_{21 / 2}-54375^{\circ 11}\end{array}$ & \\
\hline $\begin{array}{l}1953.00 \\
1957.90\end{array}$ & & 2 & $\begin{array}{l}51203.3 \\
51075.1\end{array}$ & +0.2 & $\begin{array}{l}a^{6} \mathrm{D}_{21 / 2}-54375_{21 / 2}^{1} \\
a^{6} \mathrm{D}_{11 / 2}-52593_{01 / 2}^{\circ}\end{array}$ & \\
\hline 1959.54 & & 5 & 51032.3 & +0.2 & $a^{4} \mathrm{~F}_{21 / 2}-62333_{21 / 2}^{\circ}$ & \\
\hline 1961. 42 & & 6 & 50983.5 & -0.1 & $a^{4} \mathrm{P}_{01 / 2}-59816_{11 / 2}^{\circ}$ & \\
\hline 1962.14 & & 40 & 50964.7 & 0.0 & $a^{6} \mathrm{D}_{21 / 2}-54137_{11 / 2}^{\circ}$ & \\
\hline 1962.92 & & 20 & 50944.4 & -0.3 & $a^{4} \mathrm{~F}_{31 / 2}-64356_{31 / 2}^{\circ}$ & \\
\hline 1963. 02 & & 2 & 50941.9 & -0.4 & $a^{6} \mathrm{D}_{41 / 2}-57089_{41 / 2}^{\circ}$ & \\
\hline 1967. 09 & & 2 & 50836.5 & +0.2 & $a^{6} \mathrm{D}_{11 / 2}-52355_{01 / 2}^{\circ}$ & \\
\hline 1967. 41 & & 10 & 50828.2 & +0.6 & $a^{4} \mathrm{~F}_{41 / 2}-65684^{\circ}{ }^{\circ} 1 / 2$ & \\
\hline 1968.67 & & 8 & 50795.6 & +0.1 & $a^{4} \mathrm{~F}_{31 / 2}-64207_{41 / 2}^{\circ}$ & \\
\hline $\begin{array}{l}1973.32 \\
1974.66\end{array}$ & & $\begin{array}{r}20 \\
4\end{array}$ & 50676.0 & $\begin{array}{r}0.0 \\
-0.3\end{array}$ & $a^{6} \mathrm{D}_{31 / 2}-55392_{41 / 2}^{\circ}$ & \\
\hline 1974.66 & & 4 & 50641.6 & -0.3 & $a^{4} \mathrm{G}_{51 / 2}-68078_{61 / 2}^{\circ}$ & \\
\hline 1975. 48 & & 15 & 50620.7 & -0.7 & $a^{6} \mathrm{D}_{41 / 2}-56768_{31 / 2}^{\circ}$ & \\
\hline 1976. 70 & & 25 & 50589.4 & +2.2 & $a^{6} \mathrm{~S}_{21 / 2}-58007_{11 / 2}^{\circ}$ & \\
\hline $\begin{array}{l}1977.24 \\
1977.53\end{array}$ & & $\begin{array}{l}12 \\
15\end{array}$ & $\begin{array}{l}50575.6 \\
50568.1\end{array}$ & $\begin{array}{l}+0.1 \\
-0.1\end{array}$ & $\begin{array}{l}a{ }^{4} \mathrm{G}_{51 / 2}-68012_{51 / 2}^{\circ} \\
a^{6} \mathrm{D}_{11 / 2}-52087^{\circ 11}\end{array}$ & \\
\hline $\begin{array}{l}1977.53 \\
1981.42\end{array}$ & & 20 & $\begin{array}{l}50008.1 \\
50468.8\end{array}$ & +3.2 & $a^{6} \mathrm{D}_{41 / 2}-56612^{211 / 2}$ & \\
\hline 1982.35 & & 15 & 50445. 1 & -0.9 & $a^{6} \mathrm{D}_{31 / 2}-55162_{21 / 2}^{\circ}$ & \\
\hline 1982.73 & & 1 & 50435.5 & -0.8 & $a^{6} \mathrm{~S}_{21 / 2}-57856_{21 / 2}^{0}$ & \\
\hline 1982. 92 & & 20 & 50430.7 & -0.3 & $a^{6} \mathrm{D}_{01 / 2}-50430_{11 / 2}^{1}$ & \\
\hline 1987. 80 & & 25 & 50301.9 & -4.6 & $a^{6} \mathrm{D}_{31 / 2}-55022_{31 / 2}^{\circ}$ & \\
\hline 1989. 41 & & 30 & 50266.1 & -0.4 & $a^{6} \mathrm{D}_{41 / 2}-56413_{41 / 2}^{\circ}$ & \\
\hline
\end{tabular}


TABLE 3. Classified lines of $\mathrm{W} \mathrm{II}$-Continued

\begin{tabular}{|c|c|c|c|c|c|c|}
\hline \multirow{2}{*}{$\begin{array}{l}\text { Wavelength } \\
(\AA)\end{array}$} & \multicolumn{2}{|c|}{ Intensity } & \multicolumn{2}{|c|}{ Wave number $\left(\mathrm{cm}^{-1}\right)$} & \multirow{2}{*}{ Combination } & \multirow{2}{*}{$\begin{array}{l}\text { Zeeman } \\
\text { effect }\end{array}$} \\
\hline & Arc & Spark & Observed & $O-C$ & & \\
\hline \multicolumn{7}{|l|}{ Vac. } \\
\hline 1990. 07 & & 15 & 50249.4 & $\begin{array}{l}-0.1 \\
-1.1\end{array}$ & $\begin{array}{c}a{ }^{4} \mathrm{~F}_{21 / 2}-61550_{31 / 2}^{\circ} \\
a^{6} \mathrm{D}_{21 / 2}-53422_{11 / 2}^{\circ}\end{array}$ & \\
\hline 1990. 86 & & 12 & 50229.6 & +0.3 & $a^{6} \mathrm{D}_{41 / 2}^{27 / 2}-56376_{51 / 2}^{\circ}$ & \\
\hline $\begin{array}{l}1993.15 \\
1993.53\end{array}$ & & $\begin{array}{l}6 \\
1\end{array}$ & $\begin{array}{l}50171.8 \\
50162.3\end{array}$ & $\begin{array}{l}-1.9 \\
-3.3\end{array}$ & $\begin{array}{l}a^{4} \mathrm{D}_{21 / 2}-65141_{21 / 2} \\
a^{6} \mathrm{D}_{21-53338}\end{array}$ & \\
\hline $\begin{array}{l}1993.53 \\
1993.74\end{array}$ & & 3 & $\begin{array}{l}50102.3 \\
50156.9\end{array}$ & $\begin{array}{l}-3.3 \\
-0.3\end{array}$ & $\begin{array}{l}a^{0} \mathrm{D}_{21 / 2}-53338^{11 / 2} \\
a^{6} \mathrm{D}_{21 / 2}-53329^{11 / 2}\end{array}$ & \\
\hline 1994. 21 & & 15 & 50145.1 & -0.9 & $a^{4} \mathrm{~F}_{41 / 2}-65003_{41 / 2}^{\circ}$ & \\
\hline 1995. 545 & & 25 & 50111. 6 & -0.3 & $a^{4} \mathrm{~F}_{41 / 2}-64969^{\circ}{ }^{\circ}$ & \\
\hline $\begin{array}{l}1997.46 \\
1998.54\end{array}$ & & $\begin{array}{l}6 \\
3\end{array}$ & 50063.7 & -0.3 & $a^{4} \mathrm{P}_{11 / 2}-60656_{21 / 2}^{\circ}$ & \\
\hline $\begin{array}{l}1998.54 \\
1998.99\end{array}$ & & $\begin{array}{l}5 \\
3\end{array}$ & $\begin{array}{l}50050.0 \\
50025.2\end{array}$ & $\begin{array}{r}-0.1 \\
0.0\end{array}$ & $\begin{array}{l}a^{4} \mathrm{~F}_{11 / 2}-58747^{11 / 2} \\
a^{4} \mathrm{~F}_{21 / 2}-61326_{31 / 2}^{\circ}\end{array}$ & \\
\hline \multicolumn{7}{|l|}{ Air } \\
\hline 1999. 83 & & 12 & 49988. 1 & -0.2 & $a^{6} \mathrm{D}_{31 / 2}-547042^{1} \frac{1}{2}$ & \\
\hline 2001. 70 & 3 & $\begin{array}{r}30 \\
2\end{array}$ & 49941. 4 & +0.4 & $a^{6} \mathrm{D}_{21 / 2}-53113_{21 / 2}^{\circ}$ & \\
\hline $\begin{array}{l}2002.59 \\
2002.73\end{array}$ & 1 & $\begin{array}{l}2 \\
3\end{array}$ & $\begin{array}{l}\text { 49919. } 2 \\
49915.7\end{array}$ & $\begin{array}{r}0.0 \\
+0.4\end{array}$ & $\begin{array}{c}a^{6} \mathrm{D}_{11 / 2}-514388_{21 / 2}^{\circ} \\
a^{4} \mathrm{P}_{01 / 2}-58747^{1}{ }^{11 / 2}\end{array}$ & \\
\hline 2005. 60 & & 1 & 49844.3 & +1.0 & $a^{4} \mathrm{D}_{31 / 2}-64990_{21 / 2}^{1}$ & \\
\hline 2006.07 & & 3 & 49832. 6 & +0.4 & $a^{4} \mathrm{P}_{21 / 2}-63266_{31 / 2}^{\circ}$ & \\
\hline 2008. 08 & 4 & 40 & 49782.7 & +0.5 & $a^{6} \mathrm{D}_{31 / 2}-54498_{31 / 2}^{\circ}$ & \\
\hline $\begin{array}{l}2009.37 \\
2009.98\end{array}$ & 1 & $\begin{array}{r}2 \\
12\end{array}$ & $\begin{array}{l}49750.8 \\
49735.7\end{array}$ & $\begin{array}{r}+1.6 \\
+01\end{array}$ & $a^{4} \mathrm{D}_{31 / 2}-64896_{31 / 2}^{\circ}$ & \\
\hline $\begin{array}{l}2009.98 \\
2010.24\end{array}$ & $\begin{array}{l}1 \\
3\end{array}$ & 15 & $\begin{array}{l}49735.7 \\
49729.3\end{array}$ & $\begin{array}{l}+0.1 \\
+0.1\end{array}$ & $\begin{array}{ll}a^{6} \mathrm{D}_{11 / 2} & -51254_{11 / 2}^{11} \\
a^{6} \mathrm{D}_{21 / 2} & 52901_{31 / 2}^{1}\end{array}$ & \\
\hline 2010.66 & 2 & 1 & 49718. 9 & +1.1 & $a^{4} \mathrm{G}_{41 / 2}-66270_{41 / 2}^{\circ}$ & \\
\hline 2012.18 & & 5 & 49681.3 & 0.0 & $a^{4} \mathrm{G}_{31 / 2}-66270_{41 / 2}^{\circ}$ & \\
\hline 2013. 06 & 4 & $\underline{4}$ & 49659. 6 & +0.1 & $a^{6} \mathrm{D}_{31 / 2}-54375_{21 / 2}^{\circ}$ & \\
\hline 2014. 23 & $\begin{array}{l}3 \\
3\end{array}$ & $\begin{array}{l}15 \\
10\end{array}$ & 49630.7 & +0.2 & $a^{6} \mathrm{D}_{21 / 2}-52803_{11 / 2}^{\circ}$ & \\
\hline 2014.43 & 3 & 10 & 49625.8 & +0.1 & & \\
\hline 2015. 44 & 2 & 8 & 49601. 0 & +1.1 & $a^{4} \mathrm{~F}_{21 / 2}-60900_{21 / 2}^{\circ}$ & \\
\hline $\begin{array}{l}2017.26 \\
2019.08\end{array}$ & 二 & 2 & 49556. 2 & +0.7 & $a^{4} \mathrm{P}_{21 / 2}-62989_{21 / 2}^{\circ}$ & \\
\hline $\begin{array}{l}2019.08 \\
2019.55\end{array}$ & $\overline{2}$ & $\begin{array}{l}1 \\
5\end{array}$ & $\begin{array}{l}49511.6 \\
49500.0\end{array}$ & $\begin{array}{l}-0.2 \\
+0.5\end{array}$ & $\begin{array}{l}a^{6}{ }^{6} \mathrm{~S}_{21 / 2}-56932^{11 / 2} \\
a^{4} \mathrm{~F}^{11-64556}\end{array}$ & \\
\hline $\begin{array}{l}2019.55 \\
2021.43\end{array}$ & & $\begin{array}{l}3 \\
3\end{array}$ & $\begin{array}{l}49500.0 \\
49454.0\end{array}$ & -0.6 & $\begin{array}{l}a^{4} \mathrm{~F}^{41 / 2}-64356^{01 / 1} \\
a^{6} \mathrm{~S}_{21 / 2}-568747_{421 / 2}^{\circ}\end{array}$ & \\
\hline 2023. 25 & & 1 & 49409.5 & +0.1 & $a^{4} \mathrm{G}_{21 / 2}-656443^{1 / 2}$ & \\
\hline 2023. 63 & & 3 & 49400. 3 & +0.6 & $a^{4} \mathrm{P}_{11 / 2}-59992_{21 / 2}^{\circ}$ & \\
\hline 2023. 79 & & 3 & 49396. 4 & +0.4 & $a^{4} \mathrm{D}_{11 / 2}-64030_{21 / 2}^{\circ}$ & \\
\hline 2025.45 & 1 & 4 & 49355. 9 & +0.5 & $a^{4} \mathrm{~F}_{21 / 2}-60656_{21 / 2}^{\circ}$ & \\
\hline 2025. 62 & & 2 & 49351. 7 & +1.4 & $a^{4} \mathrm{~F}_{41 / 2}-64207_{41 / 2}^{\circ}$ & \\
\hline 2026.07 & 8 & 30 & 49340.8 & +0.6 & $a^{6} \mathrm{D}_{31 / 2}-54056_{41 / 2}^{\circ}$ & \\
\hline 2027. 30 & 1 & 12 & 49310.8 & +0.9 & $a^{6} \mathrm{D}_{31 / 2}-54026_{21 / 2}$ & \\
\hline 2027.55 & 2 & 3 & 49304. 8 & +0.8 & $a^{4} \mathrm{~F}_{31 / 2}-62715_{41 / 2}^{\circ}$ & \\
\hline $\begin{array}{l}2027.87 \\
2029.12\end{array}$ & $\begin{array}{l}2 \\
1\end{array}$ & $\begin{array}{r}5 \\
15\end{array}$ & $\begin{array}{l}49296.9 \\
40266.6\end{array}$ & $\begin{array}{r}+0.6 \\
+01\end{array}$ & $a{ }^{4} \mathrm{~F}_{11 / 2}-58007_{11 / 2}$ & \\
\hline $\begin{array}{l}2029.12 \\
2029.99\end{array}$ & 5 & $\begin{array}{l}10 \\
50\end{array}$ & $\begin{array}{l}49266.6 \\
49245.5\end{array}$ & $\begin{array}{r}+0.1 \\
+0.3\end{array}$ & $\begin{array}{l}a^{4} \mathrm{G}_{51 / 2}-66703_{51 / 2}^{\circ} \\
a^{6} \mathrm{D}_{41 / 2}-55392_{41 / 2}^{\circ}\end{array}$ & \\
\hline 2030.87 & & 3 & 49224. 2 & +0.4 & $a^{4} \mathrm{P}_{11 / 2}-59816_{11 / 2}^{\circ}$ & \\
\hline 2031. 44 & & 12 & 49210. 4 & +0.7 & $a^{4} \mathrm{D}_{31 / 2}-64356^{\circ} 1 \frac{1}{2}$ & \\
\hline 2032. 16 & & 3 & 49192. 9 & +0.6 & $a^{6} \mathrm{~S}_{21 / 2}-56612_{31 / 2}^{\circ}$ & \\
\hline 2032. 92 & & 12 & 49174.5 & -0.4 & $a{ }^{4} \mathrm{P}_{01 / 2}-58007_{11 / 2}^{\circ}$ & \\
\hline 2033. 38 & & 10 & 49163. 4 & +0.4 & $a^{4} \mathrm{D}_{31 / 2}-64310_{21 / 2}^{\circ}$ & \\
\hline 2033. 73 & & 8 & 49154. 9 & +0.4 & $a^{6} \mathrm{D}_{01 / 2}-491544^{\circ 1 / 2}$ & \\
\hline 2034. 19 & & 3 & 49143.8 & -1.6 & $a^{4} \mathrm{~F}_{11 / 2}-57856_{21 / 2}^{\circ}$ & \\
\hline 2035. 02 & 5 & 25 & 49123. 8 & -0.2 & $a^{6} \mathrm{~S}_{21 / 2}-5654421 / 2$ & \\
\hline 2035.87 & 5 & 40 & 49103. 3 & +0.5 & $a^{6} \mathrm{D}_{21 / 2}-52275_{31 / 2}^{\circ}$ & \\
\hline 2036. 40 & & 2 & 49090. 5 & -0.4 & $a^{4} \mathrm{G}_{41 / 2}-6564431 / 2$ & \\
\hline 2037.58 & 3 & 40 & 49062.1 & -0.4 & $a^{4} \mathrm{D}_{21 / 2}-64030_{21 / 2}^{\circ}$ & \\
\hline
\end{tabular}


TABle 3. Classified lines of W II-Continued

\begin{tabular}{|c|c|c|c|c|c|c|}
\hline \multirow{2}{*}{$\begin{array}{c}\text { Wavelength } \\
\text { (§) }\end{array}$} & \multicolumn{2}{|c|}{ Intensity } & \multicolumn{2}{|c|}{ Wave number $\left(\mathrm{cm}^{-1}\right)$} & \multirow{2}{*}{ Combination } & \multirow{2}{*}{$\begin{array}{c}\text { Zeeman } \\
\text { effect }\end{array}$} \\
\hline & Arc & Spark & Observed & $O-C$ & & \\
\hline \multicolumn{7}{|l|}{ Air } \\
\hline $\begin{array}{l}2037.89 \\
2039.10 \\
2040.86 \\
2042.00\end{array}$ & $\frac{2}{4}$ & $\begin{array}{r}15 \\
4 \\
20 \\
6\end{array}$ & $\begin{array}{l}49054.6 \\
49025.5 \\
48983.3 \\
48956.0\end{array}$ & $\begin{array}{l}+0.3 \\
+0.4 \\
+0.4 \\
+0.6\end{array}$ & $\begin{array}{c}a^{4} \mathrm{G}_{31 / 2}-65644^{\circ}{ }^{\circ 1 / 2} \\
a^{4} \mathrm{~F}_{31 / 2}-62437^{\circ}{ }^{11 / 2} \\
a^{6} \mathrm{D}_{01 / 2}-48982^{\circ} 1 / 2 \\
a^{4} \mathrm{~F}_{21 / 2}-60256_{31 / 2}^{\circ}\end{array}$ & \\
\hline $\begin{array}{l}2042.55 \\
2043.02\end{array}$ & 2 & $\begin{array}{l}5 \\
8\end{array}$ & $\begin{array}{l}48942.7 \\
48931.3\end{array}$ & $\begin{array}{l}+0.9 \\
+0.3\end{array}$ & $\begin{array}{r}19070_{41 / 2}-68012_{51 / 2}^{\circ} \\
a^{4} \mathrm{~F}_{41 / 2}-63788_{31 / 2}^{\circ}\end{array}$ & \\
\hline $\begin{array}{l}\text { 2043. } 52 \\
2043.72 \\
2043.82 \\
2044.33 \\
\text { 2045. } 05\end{array}$ & $\begin{array}{l}2 \\
3\end{array}$ & $\begin{array}{r}30 \\
15 \\
3 \\
10 \\
3\end{array}$ & $\begin{array}{l}\text { 48919. } 6 \\
48914.7 \\
48912.3 \\
48900.1 \\
48883.0\end{array}$ & $\begin{array}{l}-1.6 \\
+0.2 \\
+0.1 \\
+1.0 \\
-0.3\end{array}$ & $\begin{array}{c}a^{4} \mathrm{H}_{31 / 2}-62333_{21 / 2}^{\circ}-52087^{\circ}{ }_{21 / 2} \\
a^{6} \mathrm{D}_{21 / 2}-50430_{11 / 2}^{\circ} \\
a^{6} \mathrm{D}_{11 / 2}-502333_{21 / 2}^{\circ} \\
a^{4} \mathrm{P}_{21 / 2}-624030_{21 / 2}^{\circ} \\
a^{4} \mathrm{D}_{31 / 2}-64\end{array}$ & \\
\hline $\begin{array}{l}2045.32 \\
2047.07 \\
2047.76 \\
\text { 2048. } 03 \\
2049.62\end{array}$ & $\begin{array}{l}2 \\
2 \\
\\
5 \\
8\end{array}$ & $\begin{array}{r}15 \\
20 \\
2 \\
30 \\
25\end{array}$ & $\begin{array}{l}48876.4 \\
48834.8 \\
48818.2 \\
48811.8 \\
48773.9\end{array}$ & $\begin{array}{l}+0.7 \\
+0.9 \\
-2.2 \\
+0.4 \\
+0.3\end{array}$ & 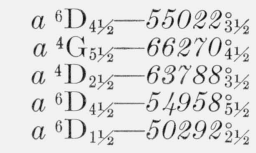 & \\
\hline $\begin{array}{l}\text { 2050. } 36 \\
2051.32\end{array}$ & $1 ?$ & $\begin{array}{r}12 \\
1\end{array}$ & $\begin{array}{l}48756.3 \\
48733.5\end{array}$ & $\begin{array}{l}+0.8 \\
+0.4\end{array}$ & $\begin{array}{l}a^{4} \mathrm{G}_{21 / 2}-64990_{21 / 2}^{\circ} \\
a^{4} \mathrm{D}_{31 / 2}-63880_{21 / 2}^{\circ}\end{array}$ & \\
\hline 2053. 10 & 5 & 15 & 48691. 3 & $\begin{array}{l}+0.2 \\
+0.8\end{array}$ & $\begin{array}{c}a^{4} \mathrm{~F}_{21 / 2}-59992_{21 / 2}^{\circ} \\
a^{6} \mathrm{D}_{21 / 2}-51863^{\circ}\end{array}$ & \\
\hline $\begin{array}{l}2053.35 \\
2054.67\end{array}$ & 10 & $\begin{array}{r}3 \\
50\end{array}$ & $\begin{array}{l}\text { 48661. } 6 \\
48654.0\end{array}$ & $\begin{array}{r}0.2 \\
+0.4\end{array}$ & $\begin{array}{ll}a^{4} \mathrm{G}_{21 / 2}-64896^{31 / 2} & -42 \\
a^{6} \mathrm{D}_{31 / 2}-53369_{41 / 2}^{3}\end{array}$ & \\
\hline $\begin{array}{l}\text { 2055. } 19 \\
\text { 2055. } 54 \\
2055.99 \\
2058.30 \\
2059.03\end{array}$ & $\begin{array}{c}2 \\
1 ? \\
2 \\
12 \\
1\end{array}$ & $\begin{array}{r}3 \\
3 \\
10 \\
15 \\
8\end{array}$ & $\begin{array}{l}48641.8 \\
48633.5 \\
48622.8 \\
48568.3 \\
48550.9\end{array}$ & $\begin{array}{l}+0.6 \\
+0.9 \\
+1.0 \\
+0.2 \\
-1.0\end{array}$ & $\begin{array}{c}a^{4} \mathrm{D}_{31 / 2}-63788_{31 / 2}^{\circ} \\
a^{4} \mathrm{~F}_{21 / 2}-59933_{31 / 2}^{\circ} \\
a^{6} \mathrm{D}_{31 / 2}-53398_{31 / 2}^{\circ}-5989_{31 / 2}^{\circ} \\
a^{4} \mathrm{~F}_{21 / 2}-595141_{21 / 2}^{\circ} \\
a^{4} \mathrm{G}_{31 / 2}-6512\end{array}$ & \\
\hline $\begin{array}{l}\text { 2059. } 45 \\
\text { 2060. } 53 \\
\text { 2063. } 34 \\
\text { 2064. } 76 \\
\text { 2065. } 06\end{array}$ & $\begin{array}{l}4 \\
3 \\
2 \\
6 A\end{array}$ & $\begin{array}{l}8 \\
2 \\
1 \\
6 \\
3\end{array}$ & $\begin{array}{l}48541.1 \\
48515.7 \\
48449.7 \\
48416.3 \\
48409.4\end{array}$ & $\begin{array}{l}+0.4 \\
+0.5 \\
-0.4 \\
+0.3 \\
+0.3\end{array}$ & 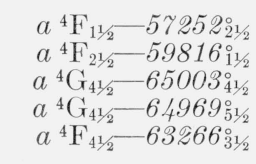 & \\
\hline $\begin{array}{l}2065.57 \\
2067.52 \\
2067.87 \\
2069.36 \\
2069.77\end{array}$ & $\begin{array}{l}3 \\
-? \\
-?\end{array}$ & $\begin{array}{r}30 \\
30 \\
10 \\
10 \\
3\end{array}$ & $\begin{array}{l}\text { 48397. } 3 \\
48351.7 \\
48343.5 \\
48308.8 \\
48299.1\end{array}$ & $\begin{array}{l}+0.1 \\
+0.3 \\
+0.4 \\
+2.2 \\
+0.6\end{array}$ & 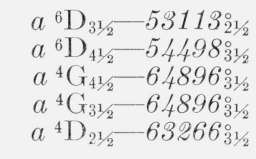 & \\
\hline $\begin{array}{l}2071.19 \\
2071.95 \\
2074.63 \\
2075.59 \\
2075.93\end{array}$ & 2 & $\begin{array}{r}40 \\
3 \\
15 \\
30 \\
10\end{array}$ & $\begin{array}{l}48265.9 \\
48248.3 \\
48185.9 \\
48163.8 \\
48155.9\end{array}$ & $\begin{array}{l}+0.4 \\
+0.5 \\
+0.5 \\
+0.1 \\
+0.5\end{array}$ & 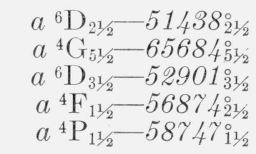 & \\
\hline $\begin{array}{l}2076.92 \\
2077.36 \\
2077.61\end{array}$ & & $\begin{array}{l}2 \\
5 \\
6\end{array}$ & $\begin{array}{l}48132.9 \\
48122.6 \\
48116.9\end{array}$ & $\begin{array}{l}+0.3 \\
+0.7 \\
+0.4\end{array}$ & $\begin{array}{l}a^{4} \mathrm{P}_{21 / 2}-61566_{21 / 2}^{\circ} \\
a^{4} \mathrm{G}_{21 / 2}-64356_{31 / 2}^{\circ}-61550_{31 / 2}^{\circ} \\
a^{4} \mathrm{P}_{21 / 2}-6150\end{array}$ & \\
\hline $\begin{array}{l}\text { 2077. } 92 \\
2078.32\end{array}$ & $\begin{array}{l}1 \\
1\end{array}$ & $\begin{array}{l}10 \\
40\end{array}$ & $\begin{array}{l}\text { 48109. } 7 \\
48100.3\end{array}$ & $\begin{array}{l}+0.4 \\
+0.7\end{array}$ & $\begin{array}{l}a^{4} \mathrm{~F}_{41 / 2}-62966_{51 / 2}^{\circ} \\
a^{4} \mathrm{P}_{01 / 2}-56932^{\circ}{ }_{11 / 2}\end{array}$ & \\
\hline 2079. 11 & 20 & 80 & 48082. 2 & $\left\{\begin{array}{l}+0.3 \\
+0.3\end{array}\right.$ & $\begin{array}{l}a^{6} \mathrm{D}_{21 / 2}-512541^{1 / 2} \\
a^{6} \mathrm{D}_{41}-54229^{\circ}{ }^{\circ} / 2\end{array}$ & \\
\hline $\begin{array}{l}\text { 2081. } 75 \\
2083.70 \\
2084.23 \\
2084.88\end{array}$ & 1 & $\begin{array}{r}3 \\
10 \\
12 \\
5\end{array}$ & $\begin{array}{l}\text { 48021. } 2 \\
47976.4 \\
47964.1 \\
47949.1\end{array}$ & $\begin{array}{r}-0.6 \\
+0.7 \\
+0.9 \\
+0.5\end{array}$ & $\begin{array}{c}a^{4} \mathrm{D}_{21 / 2}-62989^{21 / 2} \\
a^{4} \mathrm{~F}_{21 / 2}-59276_{31 / 2}^{\circ} \\
a^{4} \mathrm{G}_{41 / 2}-64516_{41 / 2}^{\circ} \\
a^{4} \mathrm{~F}_{31 / 2}-61360_{41 / 2}^{\circ}\end{array}$ & \\
\hline
\end{tabular}


Table 3. Classified lines of $\mathrm{W} \mathrm{I}$-Continued

\begin{tabular}{|c|c|c|c|c|c|c|}
\hline \multirow{2}{*}{$\begin{array}{c}\text { Wavelength } \\
(\AA)\end{array}$} & \multicolumn{2}{|c|}{ Intensity } & \multicolumn{2}{|c|}{ Wave number $\left(\mathrm{cm}^{-1}\right)$} & \multirow{2}{*}{ Combination } & \multirow{2}{*}{$\begin{array}{l}\text { Zeeman } \\
\text { effect }\end{array}$} \\
\hline & Arc & Spark & Observed & $O-C$ & & \\
\hline \multicolumn{7}{|l|}{ Air } \\
\hline $\begin{array}{l}2086.37 \\
2086.58 \\
2087.34 \\
2087.46 \\
2088.18\end{array}$ & $\begin{array}{r}2 \\
5 \\
2 \\
5 \\
60\end{array}$ & $\begin{array}{r}3 \\
4 \\
2 \\
4 \\
30\end{array}$ & $\begin{array}{l}47914.9 \\
47910.0 \\
47892.6 \\
47889.9 \\
47873.4\end{array}$ & $\begin{array}{l}+0.6 \\
+0.6 \\
+0.4 \\
+0.5 \\
+0.7\end{array}$ & $\begin{array}{l}a^{4} \mathrm{~F}_{31 / 2}-61326_{31 / 2}^{\circ} \\
a^{6} \mathrm{D}_{41 / 2}-54056_{41 / 2}^{\circ} \\
a^{4} \mathrm{P}_{21 / 2}-61326_{31 / 2}^{\circ} \\
a^{4} \mathrm{G}_{51 / 2}-65326_{51 / 2}^{\circ} \\
a^{6} \mathrm{D}_{21 / 2}-51045^{51 / 2}\end{array}$ & \\
\hline $\begin{array}{l}\text { 2089. } 13 \\
\text { 2089. } 49 \\
\text { 2091. } 23 \\
\text { 2093. } 79 \\
\text { 2094. } 72\end{array}$ & $\begin{array}{r}40 \\
2 \\
20 \\
50\end{array}$ & $\begin{array}{r}20 \\
4 \\
4 \\
20 \\
25\end{array}$ & $\begin{array}{l}47851.6 \\
47843.4 \\
47803.5 \\
47745.1 \\
47723.9\end{array}$ & $\begin{array}{l}+0.8 \\
+0.8 \\
-0.1 \\
+0.6 \\
+0.6\end{array}$ & $\begin{array}{l}a{ }^{6} \mathrm{D}_{31 / 2}-52567_{41 / 2}^{\circ} \\
a^{4} \mathrm{D}_{31 / 2}-62989_{21 / 2}^{\circ} \\
a^{4} \mathrm{G}_{41 / 2}-64356_{31 / 2}^{\circ} \\
a^{4} \mathrm{P}_{11 / 2}-58336_{21 / 2}^{\circ} \\
a^{6} \mathrm{D}_{11 / 2}^{\circ}-49242_{21 / 2}^{\circ}\end{array}$ & \\
\hline $\begin{array}{l}\text { 2095. } 78 \\
\text { 2097. } 76 \\
\text { 2098. } 22 \\
\text { 2098. } 58 \\
2098.69\end{array}$ & $\begin{array}{l}2 \\
60 A \\
70 \\
3\end{array}$ & $\begin{array}{r}1 \\
3 \\
30 \\
40 \\
\end{array}$ & $\begin{array}{l}47699.8 \\
47654.6 \\
47644.2 \\
47636.2 \\
47633.7\end{array}$ & $\begin{array}{r}+1.0 \\
+0.2 \\
+0.4 \\
+0.5 \\
+0.9\end{array}$ & $\begin{array}{r}a^{4} \mathrm{D}_{11 / 2}-62333_{21 / 2}^{\circ} \\
a^{4} \mathrm{G}_{41 / 2}-64207^{\circ 1 / 2} \\
a^{4} \mathrm{~F}_{31 / 2}-61055_{41 / 2}^{\circ} \\
a^{6} \mathrm{D}_{11 / 2}-49154^{11 / 2} \\
19070_{41 / 2}-66703^{\circ 1 / 2}\end{array}$ & \\
\hline $\begin{array}{l}\text { 2099. } 37 \\
2100.06 \\
2100.66 \\
2101.05 \\
2101.66\end{array}$ & $\frac{2}{50}+?$ & $\begin{array}{r}1 \\
1 \\
20 \\
3 \\
4\end{array}$ & $\begin{array}{l}47618.3 \\
47602.6 \\
47589.0 \\
47580.1 \\
47566.4\end{array}$ & $\begin{array}{r}+0.5 \\
+0.2 \\
+0.4 \\
+0.3 \\
+0.2\end{array}$ & $\begin{array}{c}a^{4} \mathrm{G}_{31 / 2}-64207^{\circ}{ }^{\circ} / 2 \\
a^{6} \mathrm{~S}_{21 / 2}-55022_{31 / 2}^{\circ} \\
a^{6} \mathrm{D}_{01 / 2}-47588_{11 / 2}^{\circ} \\
a^{4} \mathrm{~F}_{41 / 2}-62437^{\circ}{ }_{41 / 2} \\
a^{4} \mathrm{G}_{51 / 2}-65003_{41 / 2}^{\circ}\end{array}$ & \\
\hline $\begin{array}{l}\text { 2101. } 96 \\
2102.21 \\
2103.16 \\
2105.07 \\
2106.04\end{array}$ & $\begin{array}{r}10 \\
3 \\
30 \\
\\
2\end{array}$ & $\begin{array}{r}2 \\
1 \\
15 \\
3\end{array}$ & $\begin{array}{l}47559.6 \\
47553.9 \\
47532.5 \\
47489.3 \\
47467.4\end{array}$ & $\begin{array}{r}+0.6 \\
+0.5 \\
+0.4 \\
+0.3 \\
+0.5\end{array}$ & $\begin{array}{l}a^{6} \mathrm{D}_{31 / 2}-52275_{31 / 2}^{\circ} \\
a^{4} \mathrm{G}_{21 / 2}-63788_{31 / 2}^{\circ} \\
a^{4} \mathrm{G}_{51 / 2}-64969_{51 / 2}^{\circ} \\
a^{4} \mathrm{~F}_{31 / 2}-60900_{21 / 2}^{\circ} \\
a^{4} \mathrm{P}_{21 / 2}^{\circ} \quad 60900_{21 / 2}^{\circ}\end{array}$ & \\
\hline $\begin{array}{l}2106.17 \\
2107.26 \\
2108.38 \\
2108.65 \\
2110.33\end{array}$ & $\begin{array}{l}50 \\
\frac{5}{50} ?\end{array}$ & $\begin{array}{r}30 \\
3 \\
25 \\
15 \\
30\end{array}$ & $\begin{array}{l}47464.5 \\
47440.0 \\
47414.8 \\
47408.6 \\
47371.0\end{array}$ & $\begin{array}{l}+0.4 \\
-0.7 \\
-0.3 \\
+0.1 \\
+0.3\end{array}$ & $\begin{array}{l}a^{6} \mathrm{D}_{11 / 2}-48982^{\circ}{ }_{1 / 2} \\
a^{4} \mathrm{G}_{31 / 2}-64030_{21 / 2}^{\circ} \\
a^{4} \mathrm{P}_{11 / 2}-58007_{11 / 2}^{\circ} \\
a^{4} \mathrm{~F}_{21 / 2}-58709_{31 / 2}^{\circ} \\
a^{6} \mathrm{D}_{31 / 2}-52087_{21 / 2}^{\circ}\end{array}$ & \\
\hline 2113. 92 & 20 & 2 & 47290.5 & $\left\{\begin{array}{l}+0.1 \\
+0.5\end{array}\right.$ & $\begin{array}{l}a^{4} \mathrm{G}_{31 / 2}-63880_{21 / 2}^{\circ} \\
a^{4} \mathrm{D}_{31 / 2}-62437^{\circ 1 / 2}\end{array}$ & \\
\hline $\begin{array}{l}2114.16 \\
2115.08 \\
2115.35 \\
2115.65\end{array}$ & $\begin{array}{r}10 \\
20 \\
2\end{array}$ & $\begin{array}{l}1 \\
2\end{array}$ & $\begin{array}{l}47285.1 \\
47264.5 \\
47258.6 \\
47251.8\end{array}$ & $\begin{array}{r}+0.9 \\
+0.3 \\
+0.2 \\
+0.2\end{array}$ & $\begin{array}{c}a^{6} \mathrm{~S}_{21 / 2}-5470421 / 2 \\
a^{4} \mathrm{P}_{11 / 2}-57856_{21 / 2}^{\circ} \\
a^{6} \mathrm{D}_{21 / 2}-50430_{11 / 2}^{\circ} \\
a^{4} \mathrm{P}_{01 / 2}-56084_{11 / 2}^{\circ}\end{array}$ & \\
\hline $\begin{array}{l}2115.91 \\
2116.94 \\
2118.03 \\
2118.34 \\
2118.87\end{array}$ & $\begin{array}{l}25 \\
15 \\
40\end{array}$ & $\begin{array}{r}2 \\
2 \\
5 \\
2 \\
20\end{array}$ & $\begin{array}{l}47246.1 \\
47223.1 \\
47198.8 \\
47191.8 \\
47180.1\end{array}$ & $\begin{array}{l}+1.5 \\
+0.3 \\
+0.3 \\
+0.9 \\
+0.2\end{array}$ & $\begin{array}{l}a^{4} \mathrm{~F}_{31 / 2}-60656_{21 / 2}^{\circ} \\
a^{6} \mathrm{D}_{41 / 2}-53369_{41 / 2}^{\circ} \\
a^{4} \mathrm{G}_{31 / 2}-63788_{31 / 2}^{\circ} \\
a^{6} \mathrm{D}_{41 / 2}-53338_{31 / 2}^{\circ} \\
a^{6} \mathrm{D}_{01 / 2}-47179_{11 / 2}^{\circ}\end{array}$ & \\
\hline $\begin{array}{l}2120.38 \\
2121.60 \\
2123.42 \\
2124.14 \\
2125.58\end{array}$ & $\begin{array}{r}1 \\
100 \\
15 \\
4\end{array}$ & $\begin{array}{r}1 \\
25 \\
20 \\
1 \\
10\end{array}$ & $\begin{array}{l}47146.4 \\
47119.3 \\
47079.0 \\
47063.0 \\
47031.1\end{array}$ & $\begin{array}{l}-0.3 \\
-0.5 \\
-0.4 \\
+0.3 \\
-0.4\end{array}$ & $\begin{array}{l}a^{6} \mathrm{D}_{31 / 2}-51863_{31 / 2}^{\circ} \\
a^{6} \mathrm{D}_{21 / 2}-50292^{\circ}{ }_{21 / 2} \\
a^{4} \mathrm{G}_{51 / 2}-64516_{41 / 2}^{\circ} \\
a^{4} \mathrm{~F}_{31 / 2}-60474^{\circ}{ }^{\circ 1 / 2} \\
a^{4} \mathrm{G}_{21 / 2}-63266_{31 / 2}^{\circ}\end{array}$ & \\
\hline 2126.86 & 2 & 4 & 47002.8 & +0.3 & $18000_{31 / 2}-65003_{41 / 2}^{\circ}$ & \\
\hline 2127.44 & 12 & 20 & 46990. 0 & $\begin{array}{r}+0.4 \\
0.0\end{array}$ & $\begin{array}{r}18000_{31 / 2}-64990^{\circ} 1 / 2 \\
a^{4} \mathrm{P}_{21 / 2}-60424^{\circ}{ }^{\circ} 1 / 2\end{array}$ & \\
\hline $\begin{array}{l}2129.00 \\
2130.05 \\
2131.73\end{array}$ & $\begin{array}{r}10 \\
20 \\
4\end{array}$ & $\begin{array}{l}2 \\
2 \\
3\end{array}$ & $\begin{array}{l}46955.6 \\
46932.4 \\
46895.5\end{array}$ & $\begin{array}{r}0.0 \\
+0.2 \\
+0.1 \\
0.0\end{array}$ & $\begin{array}{r}a^{6} \mathrm{~S}_{21 / 2}-54375_{21 / 2}^{\circ} \\
a^{4} \mathrm{D}_{11 / 2}-61566_{21 / 2}^{\circ} \\
18000_{31 / 2}-64896_{31 / 2}^{\circ}\end{array}$ & \\
\hline
\end{tabular}


TABLE 3. Classified lines of $\mathrm{W} 1 \mathrm{I}-$ Continued

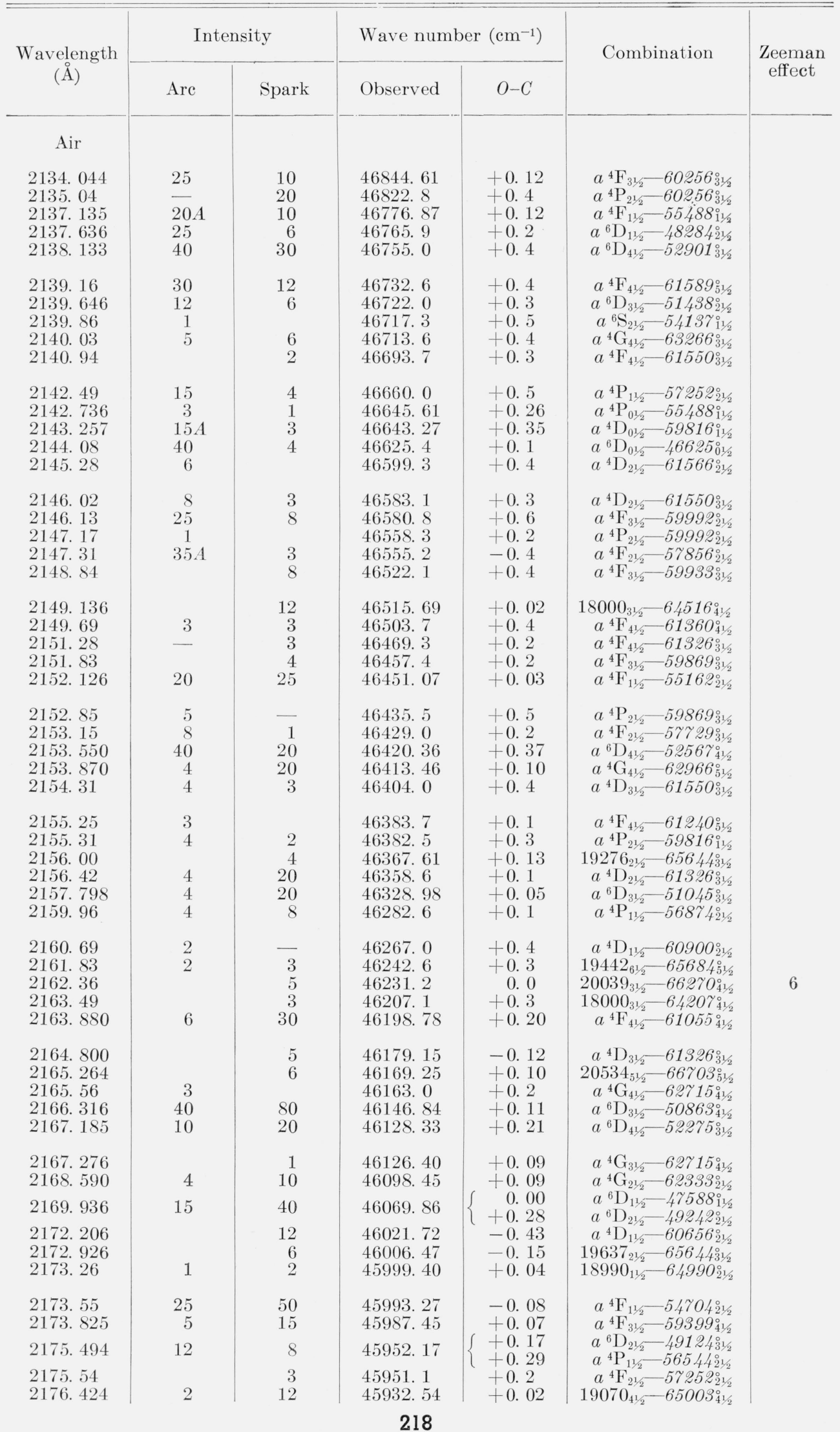


Table 3. Classified lines of $\mathrm{W}$ II--Continued

\begin{tabular}{|c|c|c|c|c|c|c|}
\hline \multirow{2}{*}{$\begin{array}{l}\text { Wavelength } \\
(\AA)\end{array}$} & \multicolumn{2}{|c|}{ Intensity } & \multicolumn{2}{|c|}{ Wave number $\left(\mathrm{cm}^{-1}\right)$} & \multirow{2}{*}{ Combination } & \multirow{2}{*}{$\begin{array}{c}\text { Zeeman } \\
\text { effect }\end{array}$} \\
\hline & Are & Spark & Observed & $O-C$ & & \\
\hline Air & & & & & & \\
\hline $\begin{array}{l}2176.88 \\
2177.13 \\
2177.52 \\
2177.546 \\
2178.04 \\
2178.72\end{array}$ & $\begin{array}{r}3 \\
3 \\
4 \\
\\
2 \\
10\end{array}$ & $\left.\begin{array}{r}15 \\
4 \\
30 \\
50\end{array}\right\}$ & $\begin{array}{l}45922.9 \\
45917.65 \\
45909.42 \\
45908.88 \\
45898.46 \\
45884.14\end{array}$ & $\begin{array}{l}-0.2 \\
+0.01 \\
+0.14 \\
+0.10 \\
+0.04 \\
+0.24\end{array}$ & 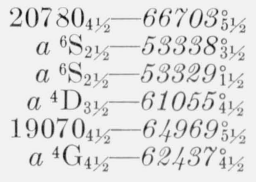 & \\
\hline 2178.92 & 10 & 30 & 45879. 9 & +0.5 & $18000_{31 / 2}-63880_{21 / 2}^{\circ}$ & \\
\hline 2179.634 & & 8 & 45864. 9 & $\begin{array}{l}+0.1 \\
-0.1\end{array}$ & 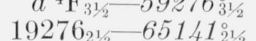 & \\
\hline $\begin{array}{l}2180.46 \\
2180.68\end{array}$ & 20 & $\begin{array}{r}40 \\
8\end{array}$ & $\begin{array}{l}45847.53 \\
45842.9\end{array}$ & $\begin{array}{l}+0.16 \\
+0.2\end{array}$ & $\begin{array}{c}a{ }^{4} \mathrm{G}_{31 / 2}-62437^{\circ}-1 / 2 \\
a^{4} \mathrm{P}_{21 / 2}-59276_{31 / 2}^{\circ}\end{array}$ & \\
\hline $\begin{array}{l}\text { 2181. } 49 \\
2182.225 \\
2183.32 \\
2183.94 \\
2185.42\end{array}$ & $\begin{array}{l}15 A \\
2 \\
-\end{array}$ & $\begin{array}{r}3 \\
40 \\
40 \\
\\
50\end{array}$ & $\begin{array}{ll}45825.9 \\
45810.45 \\
45787.48 \\
45774.48 \\
45743.49\end{array}$ & $\begin{array}{l}+0.4 \\
+0.11 \\
-0.02 \\
+0.14 \\
-0.04\end{array}$ & 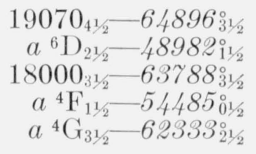 & \\
\hline $\begin{array}{l}2185.75 \\
2186.738 \\
2186.835 \\
2187.82 \\
2188.06\end{array}$ & $\begin{array}{r}5 \\
30 \\
4 \\
3\end{array}$ & $\begin{array}{r}40 \\
40 \\
10 \\
1 \\
2\end{array}$ & $\begin{array}{l}\text { 45736. } 58 \\
45715.92 \\
45713.89 \\
45693.3 \\
45688.3\end{array}$ & $\begin{array}{l}-0.02 \\
+0.05 \\
+0.09 \\
+0.2 \\
-0.4\end{array}$ & $\begin{array}{r}20534_{51 / 2}-66270_{41 / 2}^{\circ} \\
a^{6} \mathrm{D}_{41 / 2}-51863_{31 / 2}^{\circ} \\
19276_{21 / 2}-64990_{21 / 2}^{\circ} \\
a^{6} \mathrm{~S}_{21 / 2}-53113_{21 / 2}^{\circ} \\
a^{4} \mathrm{D}_{21 / 2}-60656_{21 / 2}^{\circ}\end{array}$ & \\
\hline $\begin{array}{l}\text { 2189. } 19 \\
2189.364 \\
2189.494 \\
2189.740 \\
2189.850\end{array}$ & $\begin{array}{l}30 A \\
40 \\
50 \\
6 \\
40\end{array}$ & $\begin{array}{l}30 \\
40 \\
50 \\
10 \\
80\end{array}$ & $\begin{array}{l}\text { 45664. } 7 \\
\text { 45661. } 09 \\
\text { 45658. } 38 \\
\text { 45653. } 25 \\
\text { 45650. } 96\end{array}$ & $\begin{array}{l}+0.1 \\
-0.07 \\
+0.20 \\
+0.31 \\
+0.08\end{array}$ & $\begin{array}{l}a^{4} \mathrm{~F}_{11 / 2}-54375_{21 / 2}^{\circ} \\
a^{6} \mathrm{D}_{11 / 2}-47179_{11 / 2}^{\circ} \\
a^{6} \mathrm{D}_{211 / 2}-48830_{31 / 2}^{\circ} \\
a^{4} \mathrm{P}_{01 / 2}-54485_{01 / 2}^{\circ} \\
a^{4} \mathrm{G}_{51 / 2}-63087_{61 / 2}^{\circ}\end{array}$ & \\
\hline $\begin{array}{l}\text { 2190. } 80 \\
2191.34 \\
2192.094 \\
2193.440 \\
2193.542 \\
2193.88\end{array}$ & $\begin{array}{r}4 \\
4 \\
30 \\
25\end{array}$ & $\begin{array}{r}6 \\
12 \\
6 \\
40 \\
40 \\
1\end{array}$ & $\begin{array}{l}\text { 45631. } 2 \\
\text { 45619. } 9 \\
45604.23 \\
45576.25 \\
45574.13 \\
45567.1\end{array}$ & $\begin{array}{l}0.0 \\
+0.2 \\
-0.03 \\
+0.24 \\
+0.22 \\
+0.2\end{array}$ & 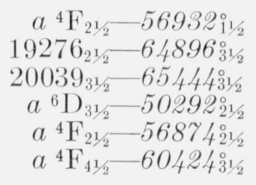 & \\
\hline $\begin{array}{l}\text { 2194. } 515 \\
2195.680 \\
2195.816 \\
2196.654 \\
2196.780\end{array}$ & 70 & $\begin{array}{r}50 \\
2 \\
25 \\
1 \\
10\end{array}$ & $\begin{array}{l}\text { 45593. } 92 \\
45529.76 \\
45526.94 \\
45509.57 \\
45506.96\end{array}$ & $\begin{array}{r}+0.22 \\
+0.28 \\
+0.38 \\
+0.08 \\
+0.11\end{array}$ & $\begin{array}{r}a^{6} \mathrm{D}_{01 / 2}-45553_{11 / 2}^{\circ} \\
a^{4} \mathrm{G}_{51 / 2}-62966_{51 / 2}^{\circ} \\
19442_{61 / 2}-64969^{\circ 1 / 2} \\
a^{4} \mathrm{D}_{31 / 2}-60656^{\circ 11 / 2} \\
a^{4} \mathrm{D}_{21 / 2}^{\circ}-6044_{421 / 2}^{\circ}\end{array}$ & \\
\hline $\begin{array}{l}2196.914 \\
2197.504 \\
2197.585 \\
2198.008 \\
2198.676\end{array}$ & $\begin{array}{l}8 \\
1\end{array}$ & $\begin{array}{r}3 \\
10 \\
\\
6 \\
60\end{array}$ & $\begin{array}{l}\text { 45504. } 18 \\
45491.97 \\
45490.3 \\
45481.54 \\
45467.72\end{array}$ & $\begin{array}{l}0.00 \\
+0.19 \\
-0.3 \\
+0.23 \\
+0.19\end{array}$ & $\begin{array}{r}19637_{21 / 2}-65141_{21 / 2}^{\circ} \\
a^{4} \mathrm{P}_{11 / 2}-56084^{\circ} 1 / 2 \\
20780_{41 / 2}-66270_{41 / 2}^{\circ} \\
a^{6} \mathrm{~S}_{21 / 2}-52901_{31 / 2}^{\circ} \\
a^{4} \mathrm{~F}_{21 / 2}-56768^{\circ}{ }^{\circ 1 / 2}\end{array}$ & \\
\hline $\begin{array}{l}\text { 2199. } 166 \\
2199.742 \\
2200.696 \\
2200.907 \\
2201.984\end{array}$ & $\begin{array}{r}40 \\
2 \\
2\end{array}$ & $\begin{array}{r}20 \\
8 \\
12 \\
12\end{array}$ & $\begin{array}{l}45457.59 \\
45445.69 \\
45426.0 \\
45421.64 \\
45399.42\end{array}$ & $\begin{array}{l}+0.57 \\
0.00 \\
+0.1 \\
+0.15 \\
+0.19\end{array}$ & $\begin{array}{r}a^{6} \mathrm{D}_{01 / 2}-45457^{\circ}{ }^{1 / 2} \\
19070_{41 / 2}-64516_{41 / 2}^{\circ} \\
a^{4} \mathrm{~F}_{11 / 2}-54137_{11 / 2}^{\circ} \\
a^{4} \mathrm{~F}_{41 / 2}-60278_{41 / 2}^{\circ} \\
a^{4} \mathrm{~F}_{41 / 2}-60256^{\circ}{ }^{\circ 1 / 2}\end{array}$ & \\
\hline $\begin{array}{l}\text { 2203. } 798 \\
2203.986 \\
2204.482 \\
2205.460 \\
2205.886\end{array}$ & $\begin{array}{r}10 \\
20 \\
100 \\
6 \\
1\end{array}$ & $\begin{array}{r}25 \\
70 \\
150 \\
8 \\
3\end{array}$ & $\begin{array}{l}45362.06 \\
45358.19 \\
45347.98 \\
45327.88 \\
45319.12\end{array}$ & $\begin{array}{r}+0.44 \\
+0.35 \\
+0.14 \\
+0.23 \\
+0.08\end{array}$ & $\begin{array}{c}a^{4} \mathrm{~F}_{41 / 2}-60218^{\circ}{ }^{1 / 2} \\
a^{4} \mathrm{D}_{11 / 2}-59992^{\circ 1 / 2} \\
a^{6} \mathrm{D}_{41 / 2}-z^{6} \mathrm{~F}_{51 / 2}^{\circ} \\
a^{4} \mathrm{D}_{31 / 2}-6047_{421 / 2} \\
18990_{11 / 2}-64310_{21 / 2}^{\circ}\end{array}$ & \\
\hline $\begin{array}{l}2206.060 \\
2206.134 \\
2206.237 \\
2206.588 \\
2206.922\end{array}$ & $\begin{array}{r}15 \\
8 \\
2 \\
40 \\
20\end{array}$ & $\begin{array}{l}15 \\
12 \\
80 \\
30\end{array}$ & $\begin{array}{l}\text { 45315. } 55 \\
\text { 45314. } 03 \\
\text { 45311. } 91 \\
\text { 45304. } 71 \\
45297.85\end{array}$ & $\begin{array}{l}-0.21 \\
+0.19 \\
+0.25 \\
+0.17 \\
+0.25\end{array}$ & $\begin{array}{l}a^{4} \mathrm{G}_{21 / 2}-61550_{31 / 2}^{\circ} \\
a^{4} \mathrm{P}_{21 / 2}-58747^{\circ}{ }_{11 / 2} \\
a^{4} \mathrm{~F}_{21 / 2}-56612_{31 / 2}^{\circ} \\
a^{4} \mathrm{P}_{01 / 2}-54137_{11 / 2}^{\circ} \\
a^{4} \mathrm{~F}_{31 / 2}-58709_{31 / 2}^{\circ}\end{array}$ & \\
\hline
\end{tabular}


TABle 3. Classified lines of $\mathrm{W} \mathrm{II}$-Continued

\begin{tabular}{|c|c|c|c|c|c|c|}
\hline \multirow{2}{*}{$\begin{array}{l}\text { Wavelength } \\
(\AA)\end{array}$} & \multicolumn{2}{|c|}{ Intensity } & \multicolumn{2}{|c|}{ Wave number $\left(\mathrm{cm}^{-1}\right)$} & \multirow{2}{*}{ Combination } & \multirow{2}{*}{$\begin{array}{c}\text { Zeeman } \\
\text { effect }\end{array}$} \\
\hline & Arc & Spark & Observed & $O-C$ & & \\
\hline \multicolumn{7}{|l|}{ Air } \\
\hline $\begin{array}{l}2207.435 \\
2207.914 \\
2207.986 \\
2208.813 \\
2209.564\end{array}$ & $\begin{array}{l}\overline{15} \\
30 \\
12\end{array}$ & $\begin{array}{r}5 \\
20 \\
40 \\
40 \\
6\end{array}$ & $\begin{array}{l}45287.33 \\
45277.50 \\
45276.02 \\
45259.07 \\
45243.69\end{array}$ & $\begin{array}{l}+1.31 \\
-0.04 \\
+0.10 \\
+0.23 \\
+0.37\end{array}$ & $\begin{array}{r}19070_{41 / 2}-64356_{31 / 2}^{\circ} \\
a^{4} \mathrm{G}_{51 / 2}^{\circ}-62714^{\circ}{ }^{\circ 1 / 2} \\
a^{4} \mathrm{~F}_{31 / 2}-58687_{41 / 2}^{\circ} \\
19637_{21 / 2}-64896_{31 / 2}^{\circ} \\
a^{4} \mathrm{~F}_{21 / 2}-56544_{21 / 2}^{\circ}\end{array}$ & \\
\hline $\begin{array}{l}2216.023 \\
2216.296 \\
2217.582 \\
2217.765\end{array}$ & $\begin{array}{r}60 \\
8 \\
4 \\
12\end{array}$ & $\begin{array}{r}40 \\
5 \\
3 \\
15\end{array}$ & $\begin{array}{l}45111.84 \\
45106.28 \\
45080.13 \\
45076.41\end{array}$ & $\begin{array}{l}-0.12 \\
-0.21 \\
-0.05 \\
-0.03\end{array}$ & $\begin{array}{r}a^{6} \mathrm{D}_{21 / 2}-48284^{\circ}{ }_{21 / 2} \\
a^{6} \mathrm{D}_{11 / 2}-46625_{01 / 2}^{\circ} \\
19276_{21 / 2}-64356_{31 / 2}^{\circ} \\
a^{4} \mathrm{~F}_{41 / 2}-59933_{31 / 2}^{\circ}\end{array}$ & res \\
\hline $\begin{array}{l}2219.588 \\
2219.740 \\
2219.882 \\
2220.938 \\
2221.525\end{array}$ & $\begin{array}{r}6 \\
10 \\
2 \\
30 \\
10\end{array}$ & $\begin{array}{r}12 \\
15 \\
2 \\
60 \\
15\end{array}$ & $\begin{array}{l}45039.39 \\
45036.31 \\
45033.42 \\
45012.01 \\
45000.12\end{array}$ & $\begin{array}{l}+0.01 \\
-0.01 \\
-0.06 \\
+0.09 \\
+0.10\end{array}$ & $\begin{array}{r}18990_{11 / 2}-64030_{21 / 2}^{\circ} \\
a^{4} \mathrm{G}_{41 / 2}-61589_{51 / 2}^{\circ} \\
19276_{21 / 2}-64310_{21 / 2}^{\circ} \\
a^{4} \mathrm{~F}_{41 / 2}-59869_{31 / 2}^{\circ} \\
a^{4} \mathrm{G}_{51 / 2}-62437_{41 / 2}^{\circ}\end{array}$ & \\
\hline $\begin{array}{l}\text { 2221. } 652 \\
2222.070 \\
2223.206 \\
2223.326 \\
2223.450\end{array}$ & $\begin{array}{r}5 \\
10 \\
2 \\
4 \\
12\end{array}$ & $\begin{array}{r}5 \\
30 \\
2 \\
6 \\
12\end{array}$ & $\begin{array}{l}44997.55 \\
44989.08 \\
44966.10 \\
44963.67 \\
44961.17\end{array}$ & $\begin{array}{l}+0.09 \\
+0.18 \\
+0.26 \\
+0.21 \\
+0.24\end{array}$ & $\begin{array}{r}a^{4} \mathrm{G}_{41 / 2}-61550_{31 / 2}^{\circ} \\
18000_{31 / 2}-62989_{21 / 2}^{\circ} \\
a^{4} \mathrm{D}_{21 / 2}-59933_{31 / 2}^{\circ} \\
20039_{31 / 2}-65003_{41 / 2}^{\circ} \\
a^{4} \mathrm{G}_{31 / 2}-61550_{31 / 2}^{\circ}\end{array}$ & \\
\hline $\begin{array}{l}2225.230 \\
2225.882 \\
2226.320 \\
2226.40 \\
2226.56\end{array}$ & $\begin{array}{l}15 A \\
60 \\
12 \\
8 \\
50\end{array}$ & $\begin{array}{r}5 \\
80 \\
15 \\
15 \\
60\end{array}$ & $\begin{array}{l}\text { 44925. } 20 \\
\text { 44912. } 05 \\
\text { 44903. } 21 \\
44901.6 \\
44898.4\end{array}$ & $\begin{array}{l}+0.18 \\
+0.42 \\
+0.33 \\
+0.3 \\
+0.3\end{array}$ & $\begin{array}{c}a^{4} \mathrm{~F}_{31 / 2}-58336_{21 / 2}^{\circ} \\
a^{6} \mathrm{D}_{01 / 2}-44911_{11 / 2}^{\circ} \\
a^{4} \mathrm{P}_{21 / 2}-58336_{21 / 2}^{\circ} \\
a^{4} \mathrm{D}_{21 / 2}-5989^{\circ} 9^{\circ 1 / 2} \\
a^{6} \mathrm{D}_{41 / 2}-51045_{31 / 2}^{\circ}\end{array}$ & res \\
\hline $\begin{array}{l}2226.68 \\
2226.77 \\
2228.29 \\
2228.70 \\
2228.88 \\
2229.026\end{array}$ & $\begin{array}{r}20 \\
20 \\
1 \\
5 \\
12 \\
10\end{array}$ & $\begin{array}{r}25 \\
70 \\
3 \\
2 \\
80 \\
6\end{array}$ & $\begin{array}{l}44895.95 \\
44894.14 \\
44863.5 \\
44855.26 \\
44851.64 \\
44848.70\end{array}$ & $\begin{array}{l}+0.46 \\
-0.1 \\
+0.41 \\
+0.22\end{array}$ & 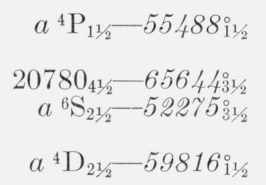 & \\
\hline $\begin{array}{l}\text { 2229. } 620 \\
2229.730 \\
2231.080 \\
2231.84 \\
2232.11\end{array}$ & $\begin{array}{r}75 \\
8 \\
12 \\
15 \\
3\end{array}$ & $\begin{array}{r}100 \\
25 \\
30 \\
5 \\
3\end{array}$ & $\begin{array}{l}44836.76 \\
44834.54 \\
44807.42 \\
44792.16 \\
44786.74\end{array}$ & $\begin{array}{l}+0.14 \\
+0.32 \\
+0.02 \\
+0.11 \\
+0.10\end{array}$ & $\begin{array}{r}a^{6} \mathrm{D}_{11 / 2}-46355_{21 / 2}^{\circ} \\
a^{4} \mathrm{D}_{01 / 2}-58007_{11 / 2}^{\circ} \\
a^{4} \mathrm{G}_{41 / 2}-61360_{41 / 2}^{\circ} \\
20534_{51 / 2}-65326_{51 / 2}^{\circ} \\
a^{4} \mathrm{D}_{31 / 2}-59933_{31 / 2}^{\circ}\end{array}$ & \\
\hline $\begin{array}{l}\text { 2232. } 284 \\
2232.56 \\
2232.80 \\
2234.63 \\
2235.00\end{array}$ & $\begin{array}{l}6 \\
4 \\
3 \\
5 ? \\
4\end{array}$ & $\begin{array}{r}4 \\
8 \\
2 \\
12 \\
2\end{array}$ & $\begin{array}{l}44783.25 \\
44777.72 \\
44772.90 \\
44736.24 \\
44728.84\end{array}$ & $\begin{array}{l}+0.03 \\
+0.09 \\
-0.25 \\
-0.38 \\
-0.07\end{array}$ & $\begin{array}{r}a^{4} \mathrm{~F}_{21 / 2}-56084{ }^{\circ 1 / 2} \\
23234_{41 / 2}-68012^{\circ}{ }^{1 / 2} \\
a^{4} \mathrm{G}_{41 / 2}-61326_{31 / 2}^{\circ} \\
a^{4} \mathrm{G}_{31 / 2}-61326_{31 / 2}^{\circ} \\
a^{4} \mathrm{~F}_{11 / 2}-53440_{01 / 2}^{\circ}\end{array}$ & \\
\hline $\begin{array}{l}2235.48 \\
2235.64 \\
2235.856 \\
2237.06 \\
2237.84\end{array}$ & $\begin{array}{r}2 \\
20 \\
8 \\
15 \\
10\end{array}$ & $\begin{array}{r}30 \\
8 \\
100 \\
4\end{array}$ & $\begin{array}{l}\text { 44719. } 24 \\
44716.03 \\
44711.71 \\
44687.65 \\
44672.08\end{array}$ & $\begin{array}{l}-0.08 \\
+0.14 \\
-0.01 \\
-0.02 \\
-0.54\end{array}$ & $\begin{array}{r}19637_{21 / 2}-64356_{31 / 2}^{\circ} \\
a^{6} \mathrm{D}_{41 / 2}-50863^{11 / 2} \\
a^{4} \mathrm{~F}_{11 / 2}^{\circ}-53422_{11 / 2}^{\circ} \\
a^{4} \mathrm{G}_{41 / 2}-61240_{51 / 2}^{\circ} \\
19637_{21 / 2}-64310_{21 / 2}^{\circ}\end{array}$ & \\
\hline $\begin{array}{l}2238.10 \\
2240.53 \\
2241.080 \\
2241.282 \\
2242.71\end{array}$ & $\begin{array}{l}2 \\
2 ? \\
30 \\
504 \\
7\end{array}$ & $\begin{array}{r}1 \\
50 \\
10 \\
7\end{array}$ & $\begin{array}{l}44666.8 \\
44618.4 \\
44607.5 \\
44603.48 \\
44575.08\end{array}$ & $\begin{array}{l}+0.2 \\
\quad 0.0 \\
0.0 \\
-0.10 \\
+0.12\end{array}$ & $\begin{array}{r}a^{6} \mathrm{~S}_{21 / 2}-52087^{\circ}{ }^{\circ 1 / 2} \\
a^{4} \mathrm{~F}_{11 / 2}-53329^{11 / 2} \\
a^{4} \mathrm{P}_{01 / 2}-53440_{01 / 2}^{\circ} \\
19276_{21 / 2}-63880_{21 / 2}^{\circ} \\
a^{4} \mathrm{~F}_{31 / 2}-57986_{41 / 2}^{\circ}\end{array}$ & 6 \\
\hline $\begin{array}{l}2242.965 \\
2244.15 \\
2244.75 \\
2245.19 \\
2245.90\end{array}$ & $\begin{array}{r}10 \\
10 \\
2 \\
25 \\
5\end{array}$ & $\begin{array}{r}12 \\
25 \\
3 \\
40 \\
3\end{array}$ & $\begin{array}{l}44570.02 \\
44546.48 \\
44534.6 \\
44525.8 \\
44511.8\end{array}$ & $\begin{array}{l}+0.24 \\
+0.46 \\
+0.2 \\
0.0 \\
+0.1\end{array}$ & $\begin{array}{r}a^{4} \mathrm{P}_{11 / 2}-55162_{21 / 2}^{\circ} \\
20780_{41 / 2}-65926^{\circ}{ }_{51 / 2} \\
20455_{11 / 2}-64990_{21 / 2}^{\circ} \\
a^{6} \mathrm{D}_{31 / 2}-49242^{\circ 112} \\
19276_{21 / 2}^{\circ}-63788^{\circ}{ }^{\circ 1 / 2}\end{array}$ & \\
\hline
\end{tabular}


TABLE 3. Classified lines of $\mathrm{W} \mathrm{II}$-Continued

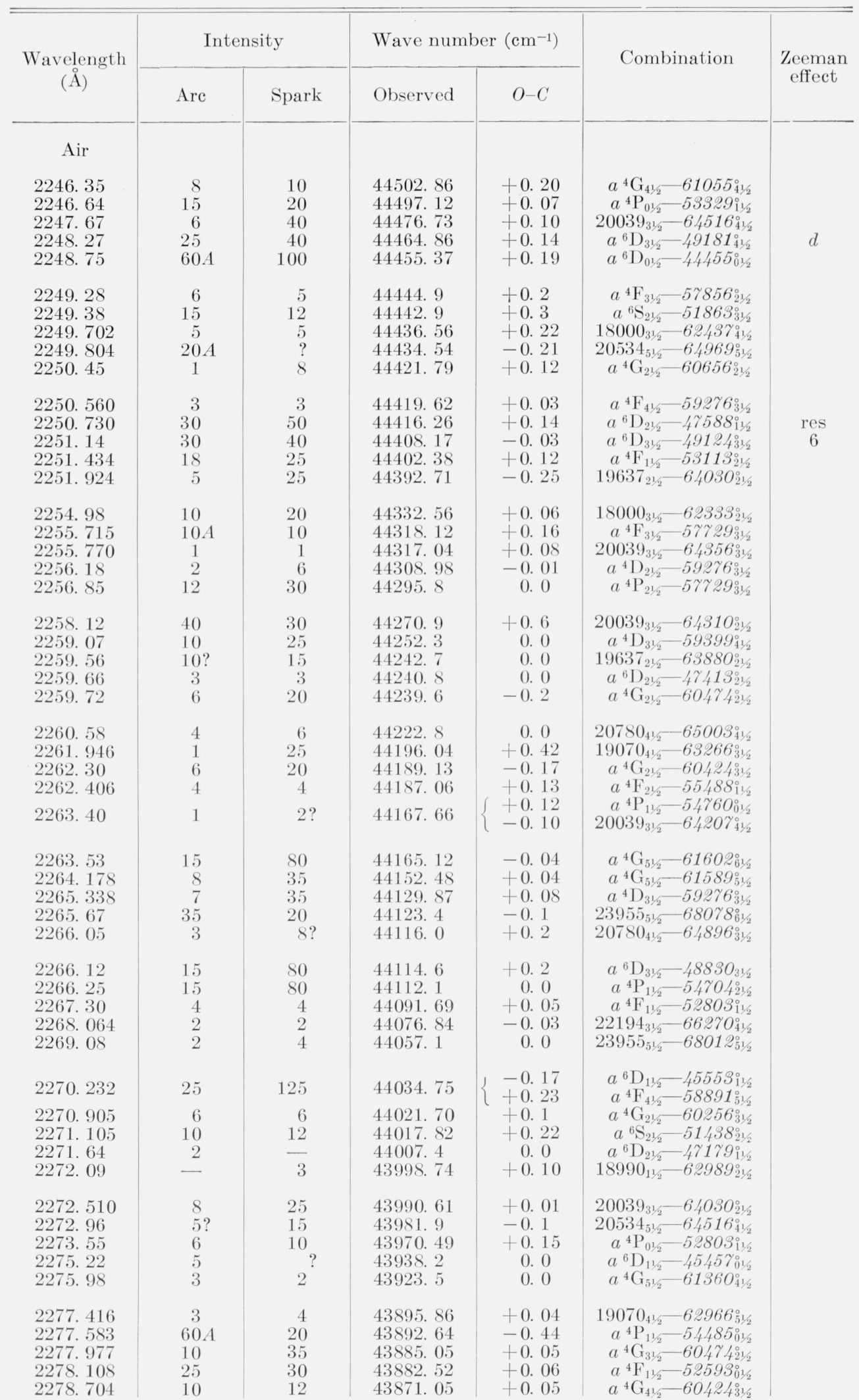


TABLE 3. Classified lines of $\mathrm{W} \mathrm{II}$-Continued

\begin{tabular}{|c|c|c|c|c|c|c|}
\hline \multirow{2}{*}{$\begin{array}{c}\text { Wavelength } \\
\text { (§) }\end{array}$} & \multicolumn{2}{|c|}{ Intensity } & \multicolumn{2}{|c|}{ Wave number $\left(\mathrm{cm}^{-1}\right)$} & \multirow{2}{*}{ Combination } & \multirow{2}{*}{$\begin{array}{c}\text { Zeeman } \\
\text { effect }\end{array}$} \\
\hline & Arc & Spark & Observed & $O-C$ & & \\
\hline Air & & & & & & \\
\hline $\begin{array}{l}2279.213 \\
2279.672 \\
2280.300 \\
2280.621 \\
2280.802\end{array}$ & $\begin{array}{l}10 \\
1- \\
8 \\
30 \\
10 ?\end{array}$ & $\begin{array}{l}3 \\
-? \\
30 \\
12 \\
10\end{array}$ & $\begin{array}{l}43861.25 \\
43852.42 \\
43840.34 \\
43834.18 \\
43830.7\end{array}$ & $\begin{array}{l}+0.03 \\
+0.08 \\
-0.02 \\
+0.21 \\
\quad 0.0\end{array}$ & $\begin{array}{r}a^{4} \mathrm{~F}_{21 / 2}-55162_{21 / 2}^{\circ}{ }^{\circ}-58709^{\circ}{ }^{11 / 2} \\
a^{4} \mathrm{~F}_{41 / 2}-58380_{21 / 2}^{\circ} \\
20039_{31 / 2}-6384_{11 / 2}^{\circ} \\
a^{6} \mathrm{~S}_{21 / 2}-51264_{41 / 2}^{\circ} \\
a^{4} \mathrm{~F}_{41 / 2}-5867^{\circ}\end{array}$ & \\
\hline $\begin{array}{l}\text { 2282. } 202 \\
2283.266 \\
2283.437 \\
2284.436 \\
2284.619\end{array}$ & $\begin{array}{l}25 \\
25 A \\
4 \\
20 \\
10\end{array}$ & $\begin{array}{r}75 \\
5 \\
3 \\
18 \\
40\end{array}$ & $\begin{array}{l}43803.81 \\
43783.40 \\
43780.12 \\
43760.98 \\
43757.47\end{array}$ & $\begin{array}{r}+0.02 \\
+0.10 \\
0.00 \\
-0.08 \\
+0.11\end{array}$ & $\begin{array}{c}a^{4} \mathrm{G}_{51 / 2}-61240_{51 / 2}^{\circ} \\
a^{4} \mathrm{P}_{11 / 2}-54375_{21 / 2}^{\circ} \\
a^{4} \mathrm{D}_{21 / 2}-58747_{11 / 2}^{\circ} \\
a^{4} \mathrm{P}_{01 / 2}-52593_{01 / 2}^{\circ} \\
a^{4} \mathrm{G}_{21 / 2}-59992_{21 / 2}^{\circ}\end{array}$ & $\begin{array}{l}6 \\
6\end{array}$ \\
\hline $\begin{array}{l}2285.09 \\
2285.75 \\
2286.28 \\
2286.476 \\
2286.94\end{array}$ & $\begin{array}{l}-? \\
1 \\
5 ? \\
6 \\
1-\end{array}$ & $\begin{array}{l}1 \\
3 \\
8 \\
4 \\
1\end{array}$ & $\begin{array}{l}43748.45 \\
43735.82 \\
43725.69 \\
43721.94 \\
43713.07\end{array}$ & $\begin{array}{l}-0.01 \\
-0.17 \\
+0.12 \\
+0.16 \\
-0.01\end{array}$ & $\begin{array}{r}20039_{31 / 2}-63788_{31 / 2}^{\circ} \\
20780_{41 / 2}-64516_{41 / 2}^{\circ} \\
a^{4} \mathrm{G}_{41 / 2}-60278_{41 / 2}^{\circ} \\
a^{4} \mathrm{~F}_{21 / 2}-5502_{31 / 2}^{\circ} \\
19276_{21 / 2}-62989_{21 / 2}^{\circ}\end{array}$ & \\
\hline $\begin{array}{l}2287.46 \\
2289.01 \\
2289.41 \\
2290.48 \\
2290.564\end{array}$ & $\begin{array}{l}8 \\
12 A \\
5 \\
2 \\
15\end{array}$ & $\begin{array}{r}5 \\
8 \\
7 \\
2 \\
20\end{array}$ & $\begin{array}{l}43703.13 \\
43673.5 \\
43665.9 \\
43645.5 \\
43643.92\end{array}$ & $\begin{array}{l}-0.18 \\
+0.3 \\
+0.2 \\
+0.1 \\
+0.07\end{array}$ & $\begin{array}{r}a^{4} \mathrm{G}_{41 / 2}-60256_{31 / 2}^{\circ} \\
20534_{51 / 2}-64207_{41 / 2}^{\circ} \\
a^{4} \mathrm{G}_{41 / 2}-60218_{51 / 2}^{\circ} \\
19442_{61 / 2}-63087_{61 / 2}^{\circ} \\
a^{4} \mathrm{~F}_{11 / 2}-52355_{01 / 2}^{\circ}\end{array}$ & \\
\hline $\begin{array}{l}2291.376 \\
2291.56 \\
2294.20 \\
2294.55 \\
2294.84\end{array}$ & $\begin{array}{l}\frac{4}{7} \\
\overline{10} \\
10\end{array}$ & $\begin{array}{r}7 \\
8 \\
3 \\
35 \\
20\end{array}$ & $\begin{array}{l}43628.45 \\
43625.0 \\
43574.7 \\
43568.1 \\
43562.6\end{array}$ & $\begin{array}{l}-0.47 \\
+0.2 \\
+0.3 \\
-0.1 \\
+0.1\end{array}$ & $\begin{array}{r}19637_{21 / 2}-63266_{31 / 2}^{\circ} \\
a^{6} \mathrm{~S}_{21 / 2}-51045^{\circ}{ }^{\circ} 1 / 2 \\
20455_{11 / 2}-64030_{21 / 2}^{\circ} \\
a^{6} \mathrm{D}_{31 / 2}-48284^{\circ}{ }^{11 / 2} \\
a^{4} \mathrm{D}_{31 / 2}-58709^{\circ}{ }^{\circ} 1 / 2\end{array}$ & res \\
\hline $\begin{array}{l}\text { 2295. } 52 \\
2295.78 \\
2295.985 \\
2296.873 \\
2296.956\end{array}$ & $\begin{array}{r}3 \\
15 \\
15 \\
4 \\
8\end{array}$ & $\begin{array}{r}3 \\
20 \\
25 \\
5 \\
6\end{array}$ & $\begin{array}{l}43549.7 \\
43544.8 \\
43540.88 \\
43524.05 \\
43522.47\end{array}$ & $\begin{array}{l}-0.2 \\
+0.1 \\
+0.02 \\
+0.09 \\
+0.02\end{array}$ & $\begin{array}{r}18000_{31 / 2}-61550_{31 / 2}^{\circ} \\
a^{4} \mathrm{P}_{11 / 2}-54137_{11 / 2}^{\circ} \\
a^{4} \mathrm{D}_{31 / 2}-58687^{11 / 2} \\
19442_{61 / 2}-62966_{51 / 2}^{\circ} \\
a^{4} \mathrm{P}_{01 / 2}-52355_{01 / 2}^{\circ}\end{array}$ & 6 \\
\hline $\begin{array}{l}\text { 2297. } 930 \\
\text { 2298. } 23 \\
\text { 2299. } 807 \\
\text { 2300. } 078 \\
\text { 2301. } 284 \\
\text { 2301. } 642\end{array}$ & $\begin{array}{r}1 \\
8 \\
6 \\
1 \\
3 \\
20\end{array}$ & $\begin{array}{r}10 \\
20 \\
18 \\
5 \\
5 \\
30\end{array}$ & $\begin{array}{l}43504.03 \\
43498.4 \\
43468.53 \\
43463.40 \\
43440.63 \\
43433.87\end{array}$ & $\begin{array}{l}0.00 \\
+0.2 \\
-0.10 \\
+0.37 \\
-0.26 \\
+0.15\end{array}$ & $\begin{array}{r}22140_{21 / 2}-65644_{431 / 2}^{\circ} \\
a^{4} \mathrm{P}_{21 / 2}-56932_{11 / 2}^{\circ} \\
23234_{41 / 2}-66703_{51 / 2}^{\circ} \\
a^{4} \mathrm{~F}_{31 / 2}-56877^{\circ}{ }^{11 / 2} \\
a^{4} \mathrm{P}_{21 / 2}-5687^{\circ} 2^{1 / 2} \\
a^{4} \mathrm{P}_{11 / 2}-54026_{21 / 2}^{\circ}\end{array}$ & res \\
\hline $\begin{array}{l}\text { 2302. } 139 \\
\text { 2303. } 274 \\
2303.819 \\
\text { 2304. } 474 \\
\text { 2305. } 219\end{array}$ & $\begin{array}{l}\overline{25} \\
25 \\
\overline{4}\end{array}$ & $\begin{array}{r}1 \\
50 \\
75 \\
2 \\
8\end{array}$ & $\begin{array}{l}43424.49 \\
43403.10 \\
43392.83 \\
43380.50 \\
43366.48\end{array}$ & $\begin{array}{l}+0.32 \\
+0.57 \\
-0.02 \\
-0.02 \\
+0.12\end{array}$ & $\begin{array}{r}20455_{11 / 2}-63880_{21 / 2}^{\circ} \\
a^{4} \mathrm{G}_{31 / 2}-59992_{21 / 2}^{\circ} \\
a^{6} \mathrm{D}_{11 / 2}-44911_{11 / 2}^{11} \\
a^{4} \mathrm{G}_{41 / 2}-59933_{31 / 2}^{\circ} \\
19070_{41 / 2}-62437_{41 / 2}^{\circ}\end{array}$ & res \\
\hline $\begin{array}{l}2305.553 \\
2305.705 \\
2305.972 \\
2306.419 \\
2306.511\end{array}$ & $\begin{array}{l}- \\
\overline{1} \\
2 \\
-?\end{array}$ & $\begin{array}{l}1 \\
1 \\
1 \\
3 \\
4\end{array}$ & $\begin{array}{l}43360.20 \\
43357.34 \\
43352.32 \\
43343.92 \\
43342.19\end{array}$ & $\begin{array}{l}+0.36 \\
+0.69 \\
+0.10 \\
-0.07 \\
-0.05\end{array}$ & $\begin{array}{r}18000_{31 / 2}-61360_{41 / 2}^{\circ} \\
a^{4} \mathrm{~F}_{31 / 2}-56768_{31 / 2}^{\circ} \\
19637_{21 / 2}-62989_{21 / 2}^{\circ} \\
a^{4} \mathrm{G}_{31 / 2}-59933_{31 / 2}^{\circ} \\
18990_{11 / 2}-62333_{21 / 2}^{\circ}\end{array}$ & \\
\hline $\begin{array}{l}\text { 2306. } 918 \\
2307.392 \\
2307.930 \\
2309.850 \\
2310.244\end{array}$ & $\begin{array}{r}15 \\
2 \\
5 \\
8 \\
4\end{array}$ & $\begin{array}{r}40 \\
3 \\
10 \\
25 \\
12\end{array}$ & $\begin{array}{l}43334.55 \\
43325.64 \\
43315.54 \\
43279.54 \\
43272.16\end{array}$ & $\begin{array}{l}+0.04 \\
+0.05 \\
-0.46 \\
+0.07 \\
+0.14\end{array}$ & $\begin{array}{r}a^{4} \mathrm{P}_{21 / 2}-56768_{31 / 2}^{\circ} \\
18000_{31 / 2}-61326_{31 / 2}^{\circ} \\
a^{4} \mathrm{G}_{41 / 2}-59869_{31 / 2}^{\circ} \\
a^{4} \mathrm{G}_{31 / 2}-59869_{31 / 2}^{\circ} \\
19442_{61 / 2}-627146^{\circ 112}\end{array}$ & res \\
\hline $\begin{array}{l}\text { 2312. } 800 \\
\text { 2312. } 907 \\
\text { 2314. } 084 \\
\text { 2314. } 637 \\
2315.022\end{array}$ & $\begin{array}{l}\overline{6} \\
\overline{10} \\
20\end{array}$ & $\begin{array}{r}4 \\
20 \\
6 \\
25 \\
50\end{array}$ & $\begin{array}{l}43224.35 \\
43222.35 \\
43200.36 \\
43190.04 \\
43182.87\end{array}$ & $\begin{array}{l}+0.20 \\
+0.01 \\
-0.42 \\
+0.08 \\
-0.01\end{array}$ & $\begin{array}{r}23046_{31 / 2}-66270_{41 / 2}^{\circ} \\
a^{4} \mathrm{D}_{11 / 2}-57856_{21 / 2}^{\circ} \\
a^{4} \mathrm{~F}_{31 / 2}-56612_{31 / 2}^{\circ} \\
a^{4} \mathrm{D}_{31 / 2}^{\circ}-58336_{21 / 2}^{\circ} \\
a^{6} \mathrm{D}_{21 / 2}^{\circ}-46355_{21 / 2}^{\circ}\end{array}$ & \\
\hline
\end{tabular}


TABLE 3. Classified lines of $\mathrm{W} \mathrm{II}$-Continued

\begin{tabular}{|c|c|c|c|c|c|c|}
\hline \multirow{2}{*}{$\begin{array}{l}\text { Wavelength } \\
(\AA)\end{array}$} & \multicolumn{2}{|c|}{ Intensity } & \multicolumn{2}{|c|}{ Wave number $\left(\mathrm{cm}^{-1}\right)$} & \multirow{2}{*}{ Combination } & \multirow{2}{*}{$\begin{array}{c}\text { Zeeman } \\
\text { effect }\end{array}$} \\
\hline & Arc & Spark & Observed & $O-C$ & & \\
\hline \multicolumn{7}{|l|}{ Air } \\
\hline $\begin{array}{l}2315.246 \\
2317.722 \\
2317.88 \\
2318.916 \\
2320.832\end{array}$ & $\begin{array}{l}3 \\
4 \\
6 \\
3 \\
6\end{array}$ & $\begin{array}{r}4 \\
6 \\
10 \\
6 \\
5\end{array}$ & $\begin{array}{l}43178.69 \\
43132.57 \\
43129.62 \\
43110.36 \\
43074.76\end{array}$ & $\begin{array}{l}+0.05 \\
+0.13 \\
-0.08 \\
+0.06 \\
+0.02\end{array}$ & $\begin{array}{l}a^{4} \mathrm{P}_{21 / 2}-56612_{31 / 2}^{\circ} \\
a^{4} \mathrm{~F}_{31 / 2}-5654421 / 2 \\
a^{4} \mathrm{~F}_{41 / 2}-57986_{41 / 2}^{\circ} \\
a^{4} \mathrm{P}_{21 / 2}-5654421 / 2 \\
a^{4} \mathrm{~F}_{21 / 2}-54375_{21 / 2}^{\circ}\end{array}$ & \\
\hline $\begin{array}{l}2321.807 \\
2322.594 \\
2322.720 \\
2323.038 \\
2324.291\end{array}$ & $\begin{array}{r}- \\
2 \\
5 \\
15 \\
7\end{array}$ & $\begin{array}{r}1 \\
3 \\
8 \\
25 \\
5\end{array}$ & $\begin{array}{l}43056.68 \\
43042.10 \\
43039.76 \\
43033.87 \\
43010.67\end{array}$ & $\begin{array}{r}0.00 \\
+0.13 \\
-0.02 \\
-0.01 \\
+0.15\end{array}$ & $\begin{array}{r}19276_{21 / 2}-62333^{\circ}{ }^{\circ 1 / 2} \\
a^{4} \mathrm{G}_{21 / 2}-59276_{31 / 2}^{\circ} \\
a^{4} \mathrm{D}_{21 / 2}-58007^{\circ}{ }_{11 / 2} \\
a^{6} \mathrm{D}_{41 / 2}-49181_{41 / 2}^{\circ} \\
a^{6} \mathrm{~S}_{21 / 2}-50430_{11 / 2}^{\circ}\end{array}$ & 6 \\
\hline 2324. 714 & 1 & 1 & 43002.84 & -0.06 & $a^{6} \mathrm{D}_{21 / 2}-46175_{31 / 2}^{\circ}$ & \\
\hline 2324. 776 & 3 & 10 & 43001.70 & $\begin{array}{l}+0.02 \\
+0.11\end{array}$ & $\begin{array}{r}a^{4} \mathrm{~F}_{31 / 2}-56413_{41 / 2}^{\circ} \\
22139^{\circ}-65141^{\circ}-6\end{array}$ & \\
\hline $\begin{array}{l}\text { 2326. } 091 \\
2327.592 \\
2327.737\end{array}$ & $\begin{array}{l}15 \\
8 A \\
-\end{array}$ & $\begin{array}{r}60 \\
5 \\
1\end{array}$ & $\begin{array}{l}42977.38 \\
42949.67 \\
42947.0\end{array}$ & $\begin{array}{l}+0.02 \\
-0.19 \\
-0.5\end{array}$ & $\begin{array}{c}a^{6} \mathrm{D}_{41 / 2}-49124_{31 / 2}^{\circ} \\
20039_{31 / 2}-62989_{21 / 2}^{\circ} \\
22194_{31 / 2}-65141_{21 / 2}^{\circ}\end{array}$ & \\
\hline $\begin{array}{l}\text { 2328. } 314 \\
2329.691 \\
2330.89 \\
2331.778 \\
2331.816\end{array}$ & $\begin{array}{r}20 \\
6 \\
5 \\
3 \\
1\end{array}$ & $\begin{array}{r}35 \\
15 \\
8 \\
4 \\
1\end{array}$ & $\begin{array}{l}42936.35 \\
42910.98 \\
42888.91 \\
42872.57 \\
42871.87\end{array}$ & $\begin{array}{l}-0.05 \\
+0.06 \\
+0.03 \\
-0.13 \\
-0.03\end{array}$ & $\begin{array}{c}a^{6} \mathrm{D}_{11 / 2}-44455_{01 / 2}^{\circ} \\
a^{4} \mathrm{D}_{01 / 2}-56084_{11 / 2} \\
a^{4} \mathrm{D}_{21 / 2}-57856_{21 / 2}^{\circ} \\
a^{4} \mathrm{~F}_{41 / 2}-57799_{31 / 2}^{\circ} \\
a^{6} \mathrm{~S}_{21 / 2}-50292_{21 / 2}^{\circ}\end{array}$ & res \\
\hline $\begin{array}{l}\text { 2333. } 146 \\
2333.462 \\
2333.57 \\
2333.770 \\
2334.070\end{array}$ & $\begin{array}{r}25 A \\
3 \\
15 \\
5\end{array}$ & $\begin{array}{r}12 \\
1 \\
8 \\
35 \\
5\end{array}$ & $\begin{array}{l}42847.44 \\
42841.64 \\
42839.66 \\
42835.98 \\
42830.48\end{array}$ & $\begin{array}{l}-0.21 \\
-0.05 \\
+0.24 \\
-0.06 \\
+0.02\end{array}$ & $\begin{array}{l}a^{4} \mathrm{P}_{11 / 2}-53440_{01 / 2}^{\circ} \\
a^{4} \mathrm{G}_{51 / 2}-60278_{41 / 2}^{11} \\
a^{4} \mathrm{D}_{31 / 2}-57986_{41 / 2}^{\circ} \\
a^{6} \mathrm{D}_{11 / 2}-44354211 / 2 \\
a^{4} \mathrm{P}_{11 / 2}-53422_{11 / 2}^{\circ}\end{array}$ & res \\
\hline $\begin{array}{l}\text { 2335. } 205 \\
\text { 2335. } 548 \\
2335.933 \\
2336.716 \\
2337.799\end{array}$ & $\begin{array}{l}6 \\
2 \\
6 \\
6\end{array}$ & $\begin{array}{r}25 \\
5 \\
8 \\
15 \\
20\end{array}$ & $\begin{array}{l}42809.66 \\
42803.38 \\
42796.32 \\
42781.98 \\
42762.17\end{array}$ & $\begin{array}{l}-0.01 \\
+0.16 \\
+0.08 \\
+0.16 \\
+0.07\end{array}$ & $\begin{array}{r}a^{4} \mathrm{G}_{31 / 2}-59399_{41 / 2}^{\circ} \\
25209_{41 / 2}-68012_{51 / 2}^{\circ} \\
22194_{31 / 2}-64990_{21 / 2}^{\circ} \\
a^{4} \mathrm{G}_{51 / 2}-60218_{51 / 2}^{\circ} \\
a^{4} \mathrm{D}_{21 / 2}-57729_{31 / 2}^{\circ}\end{array}$ & \\
\hline $\begin{array}{l}2338.125 \\
2338.582 \\
2339.16 \\
2339.732 \\
2339.817\end{array}$ & $\begin{array}{r}-? \\
15 \\
10 \\
1\end{array}$ & $\begin{array}{r}2 \\
1 \\
30 \\
8 \\
?\end{array}$ & $\begin{array}{l}\text { 42756. } 21 \\
42747.86 \\
42737.29 \\
42726.84 \\
42725.29\end{array}$ & $\begin{array}{l}-0.04 \\
-0.24 \\
+0.10 \\
+0.07 \\
+0.13\end{array}$ & $\begin{array}{r}22139_{21 / 2}-64896_{31 / 2}^{\circ} \\
23955_{51 / 2}-66703_{51 / 2}^{\circ} \\
a^{4} \mathrm{P}_{11 / 2}-53329_{11 / 2}^{\circ} \\
a^{4} \mathrm{~F}_{11 / 2}-51438_{21 / 2}^{\circ} \\
a^{4} \mathrm{~F}_{21 / 2}-54026_{21 / 2}^{\circ}\end{array}$ & \\
\hline $\begin{array}{l}2339.904 \\
2340.668 \\
2341.074 \\
2341.368 \\
2341.442\end{array}$ & $\begin{array}{r}6 \\
4 \\
4 \\
25 \\
1-\end{array}$ & $\begin{array}{c}20 \\
6 \\
15 \\
35 \\
2 ?\end{array}$ & $\begin{array}{l}42723.70 \\
42709.76 \\
42702.35 \\
42696.99 \\
42695.64\end{array}$ & $\begin{array}{l}+0.03 \\
+0.08 \\
+0.21 \\
-0.02 \\
-0.18\end{array}$ & $\begin{array}{r}a^{4} \mathrm{G}_{41 / 2}-59276_{31 / 2}^{\circ} \\
a^{4} \mathrm{D}_{31 / 2}-57856_{21 / 2}^{\circ} \\
22194_{31 / 2}-64896_{31 / 2}^{\circ} \\
a^{6} \mathrm{D}_{31 / 2}-47413_{21 / 2}^{\circ} \\
19637_{21 / 2}^{\circ}-62393_{21 / 2}^{\circ}\end{array}$ & res \\
\hline $\begin{array}{l}2341.903 \\
2346.864 \\
2347.644 \\
2348.015 \\
2349.262 \\
2349.839\end{array}$ & $\begin{array}{l}1 \\
1 \\
1 \\
3 \\
6\end{array}$ & $\begin{array}{r}6 \\
5 \\
3 \\
7 \\
20 \\
10\end{array}$ & $\begin{array}{l}\text { 42687. } 24 \\
42597.01 \\
42582.86 \\
42576.13 \\
42553.53 \\
42543.09\end{array}$ & $\begin{array}{l}+0.10 \\
-0.15 \\
-0.04 \\
+0.39 \\
-0.02 \\
-0.05\end{array}$ & $\begin{array}{r}a^{4} \mathrm{G}_{31 / 2}-59276_{31 / 2}^{\circ} \\
23046_{31 / 2}-65644^{\circ}{ }^{\circ 1 / 2} \\
a^{4} \mathrm{D}_{31 / 2}-57729_{31 / 2}^{\circ} \\
18990_{11 / 2}-61566_{21 / 2}^{\circ} \\
20534_{51 / 2}^{\circ}-63087_{61 / 2}^{\circ} \\
a^{4} \mathrm{~F}_{11 / 2}-51254_{11 / 2}^{\circ}\end{array}$ & 6 \\
\hline $\begin{array}{l}2350.362 \\
2351.052 \\
2351.168 \\
2351.496 \\
2352.924\end{array}$ & $\begin{array}{l}2 \\
2 \\
1- \\
4\end{array}$ & $\begin{array}{r}10 \\
6 \\
1 \\
12 \\
4\end{array}$ & $\begin{array}{l}\text { 42533. } 62 \\
42521.14 \\
\text { 42519. } 04 \\
42513.11 \\
42487.31\end{array}$ & $\begin{array}{l}-0.05 \\
+0.14 \\
+0.26 \\
+0.01 \\
+0.07\end{array}$ & $\begin{array}{r}20455_{11 / 2}-62989_{21 / 2}^{\circ} \\
a^{4} \mathrm{P}_{11 / 2}-53113_{21 / 2}^{\circ} \\
19070_{41 / 2}-61589_{51 / 2}^{\circ} \\
a^{4} \mathrm{G}_{21 / 2}-58747_{11 / 2}^{\circ} \\
22503_{11 / 2}-64990_{21 / 2}^{\circ}\end{array}$ & \\
\hline $\begin{array}{l}\text { 2353. } 334 \\
2353.667 \\
2354.018 \\
2355.019 \\
2356.469\end{array}$ & $\begin{array}{l}2 \\
1\end{array}$ & $\begin{array}{l}8 \\
5 \\
2 \\
4 \\
2\end{array}$ & $\begin{array}{l}42479.91 \\
42473.90 \\
42467.57 \\
42449.52 \\
42423.40\end{array}$ & $\begin{array}{l}-0.01 \\
-0.07 \\
+0.46 \\
-0.41 \\
-0.04\end{array}$ & $\begin{array}{l}19070_{41 / 2}-61550_{31 / 2}^{\circ} \\
18000_{31 / 2}-6047421 / 2 \\
23803_{31 / 2}-66270_{41 / 2}^{\circ} \\
23234_{41 / 2}-65684^{\circ}{ }^{\circ 1 / 2} \\
18000_{31 / 2}-60424^{\circ} 1 \frac{1}{2}\end{array}$ & \\
\hline
\end{tabular}


TABLE 3. Classified lines of W II-Continued

\begin{tabular}{|c|c|c|c|c|c|c|}
\hline \multirow{2}{*}{$\begin{array}{l}\text { Wavelength } \\
(\AA)\end{array}$} & \multicolumn{2}{|c|}{ Intensity } & \multicolumn{2}{|c|}{ Wave number $\left(\mathrm{cm}^{-1}\right)$} & \multirow{2}{*}{ Combination } & \multirow{2}{*}{$\begin{array}{c}\text { Zeeman } \\
\text { effect }\end{array}$} \\
\hline & Arc & Spark & Observed & $O-C$ & & \\
\hline \multicolumn{7}{|l|}{ Air } \\
\hline $\begin{array}{l}\text { 2357. } 268 \\
2357.931 \\
2358.816 \\
2361.194 \\
\text { 2362. } 108 \\
\text { 2362. } 484\end{array}$ & $\begin{array}{l}1 \\
12 \\
12 A \\
3 \\
1\end{array}$ & $\begin{array}{r}5 \\
1 \\
18 \\
10 \\
15 \\
10\end{array}$ & $\begin{array}{l}42409.02 \\
42397.10 \\
42381.19 \\
42338.51 \\
42322.13 \\
42315.40\end{array}$ & $\begin{array}{l}-0.11 \\
-0.20 \\
+0.01 \\
-0.09 \\
-0.16 \\
-0.15\end{array}$ & $\begin{array}{r}23234_{41 / 2}-65644_{411 / 2}^{\circ} \\
20039_{31 / 2}-6243^{\circ} 7_{41 / 2}^{\circ} \\
a^{6} \mathrm{D}_{21 / 2}-45553_{11 / 2}^{\circ} \\
a^{4} \mathrm{G}_{41 / 2}-58891_{51 / 2}^{\circ} \\
22194_{31 / 2}-64516_{41 / 2}^{\circ} \\
23955_{51 / 2}^{\circ}-66270_{41 / 2}^{\circ}\end{array}$ & res \\
\hline $\begin{array}{l}\text { 2362. } 528 \\
2363.464 \\
2363.721 \\
2363.910 \\
\text { 2364. } 225\end{array}$ & $\begin{array}{r}1 \\
8 \\
6 \\
10\end{array}$ & $\begin{array}{r}4 ? \\
14 \\
1 \\
30 \\
50\end{array}$ & $\begin{array}{l}42314.61 \\
42297.85 \\
42293.25 \\
42289.87 \\
42284.24\end{array}$ & $\begin{array}{l}-0.02 \\
-0.06 \\
-0.21 \\
+0.01 \\
+0.06\end{array}$ & $\begin{array}{r}a^{4} \mathrm{D}_{01 / 2}-55488_{11 / 2}^{\circ} \\
a^{4} \mathrm{D}_{11 / 2}-56932_{11 / 2}^{\circ} \\
20039_{31 / 2}-62333_{21 / 2}^{\circ} \\
19070_{41 / 2}-61360_{41 / 2}^{\circ} \\
a^{4} \mathrm{D}_{21 / 2}-57252_{21 / 2}^{\circ}\end{array}$ & \\
\hline $\begin{array}{l}\text { 2364. } 577 \\
2365.832 \\
2366.684 \\
2367.132 \\
2368.021\end{array}$ & $\begin{array}{l}3 \\
4 \\
1\end{array}$ & $\begin{array}{l}1 \\
7 \\
6 \\
3 \\
2\end{array}$ & $\begin{array}{l}42277.94 \\
42255.52 \\
42240.31 \\
42232.32 \\
42216.46\end{array}$ & $\begin{array}{l}-0.07 \\
-0.09 \\
-0.32 \\
+0.08 \\
-0.27\end{array}$ & $\begin{array}{r}18000_{31 / 2}-6027_{41 / 2}^{\circ} \\
19070_{41 / 2}-61326_{31 / 2}^{\circ} \\
a^{4} \mathrm{D}_{11 / 2}-5687_{421 / 2}^{\circ} \\
a^{4} \mathrm{~F}_{41 / 2}-57089_{41 / 2}^{\circ} \\
22139_{21 / 2}^{\circ}-64356_{31 / 2}^{\circ}\end{array}$ & \\
\hline $\begin{array}{l}\text { 2368. } 36 \\
2369.755 \\
2370.056 \\
2370.624 \\
2371.058\end{array}$ & $\begin{array}{r}8 \\
6 \\
10 \\
5 \\
1\end{array}$ & $\begin{array}{r}14 \\
8 \\
45 \\
12 \\
7\end{array}$ & $\begin{array}{l}42210.42 \\
42185.57 \\
42180.22 \\
42170.11 \\
42162.40\end{array}$ & $\begin{array}{r}-0.06 \\
0.00 \\
+0.01 \\
-0.02 \\
-0.22\end{array}$ & $\begin{array}{r}a^{4} \mathrm{P}_{11 / 2}-52803_{11 / 2}^{\circ} \\
a^{6} \mathrm{D}_{41 / 2}-48332_{51 / 2}^{\circ} \\
20534_{51 / 2}-627146^{\circ 1 / 2} \\
19070_{41 / 2}^{\circ}-61240_{51 / 2}^{\circ} \\
22194_{31 / 2}^{\circ}-64356_{31 / 2}^{\circ}\end{array}$ & \\
\hline $\begin{array}{l}\text { 2371. } 218 \\
2371.936 \\
2372.610 \\
\text { 2373. } 305 \\
\text { 2373. } 462\end{array}$ & $\begin{array}{l}2 \\
5 \\
5\end{array}$ & $\begin{array}{r}5 \\
20 \\
30 \\
1 \\
4\end{array}$ & $\begin{array}{l}42159.55 \\
42146.79 \\
42134.82 \\
42122.48 \\
42119.69\end{array}$ & $\begin{array}{l}-0.09 \\
-0.13 \\
+0.08 \\
+0.58 \\
-0.20\end{array}$ & $\begin{array}{r}19442_{61 / 2}-61602^{\circ}{ }^{\circ 1 / 2} \\
19442_{61 / 2}-61589_{51 / 2}^{\circ} \\
a^{4} \mathrm{G}_{41 / 2}-58687^{\circ}{ }^{11 / 2} \\
a^{4} \mathrm{~F}_{21 / 2}-53422_{11 / 2}^{\circ} \\
a^{4} \mathrm{G}_{31 / 2}-58709^{\circ}{ }^{11 / 2}\end{array}$ & \\
\hline $\begin{array}{l}\text { 2373. } 679 \\
2374.454 \\
2375.044 \\
2377.172 \\
2377.393\end{array}$ & $\begin{array}{l}1 \\
5 ? \\
4 \\
6 \\
6\end{array}$ & $\begin{array}{r}3 \\
35 \\
20 \\
12 \\
8\end{array}$ & $\begin{array}{l}42115.84 \\
42102.10 \\
42091.64 \\
42053.96 \\
42050.06\end{array}$ & $\begin{array}{l}-0.08 \\
-0.04 \\
+0.11 \\
+0.05 \\
+0.29\end{array}$ & $\begin{array}{r}22194_{31 / 2}-64310_{21 / 2}^{\circ} \\
a^{4} \mathrm{G}_{21 / 2}-58336_{21 / 2}^{\circ} \\
23234_{41 / 2}-65326_{51 / 2}^{\circ} \\
a^{4} \mathrm{P}_{21 / 2}-55488_{11 / 2}^{\circ} \\
19276_{21 / 2}^{\circ}-61326_{31 / 2}^{\circ}\end{array}$ & res \\
\hline $\begin{array}{l}\text { 2378. } 130 \\
2378.603 \\
2379.466 \\
2380.159 \\
2380.708\end{array}$ & $\begin{array}{r}8 \\
10 \\
1 \\
4 \\
3\end{array}$ & $\begin{array}{r}15 \\
15 \\
2 \\
9 \\
5\end{array}$ & $\begin{array}{l}42037.02 \\
42028.67 \\
42013.42 \\
42001.19 \\
41991.51\end{array}$ & $\begin{array}{r}+0.03 \\
+0.04 \\
0.00 \\
-0.01 \\
+0.01\end{array}$ & $\begin{array}{r}a^{4} \mathrm{~F}_{21 / 2}-53338_{31 / 2}^{\circ} \\
a^{4} \mathrm{~F}_{21 / 2}-53329_{11 / 2}^{\circ} \\
22194_{31 / 2}-64207_{41 / 2}^{\circ} \\
a^{4} \mathrm{P}_{11 / 2}-52593_{01 / 2}^{\circ} \\
18000_{31 / 2}-59992_{21 / 2}^{\circ}\end{array}$ & \\
\hline $\begin{array}{l}\text { 2381. } 065 \\
2381.333 \\
2382.243 \\
2382.364 \\
2382.700\end{array}$ & $\begin{array}{l}1 \\
6 \\
2 \\
7\end{array}$ & $\begin{array}{r}2 \\
8 \\
2 \\
20 \\
25\end{array}$ & $\begin{array}{l}\text { 41985. } 22 \\
41980.47 \\
41964.45 \\
41962.32 \\
41956.40\end{array}$ & $\begin{array}{r}+0.10 \\
+0.06 \\
0.00 \\
0.00 \\
0.00\end{array}$ & $\begin{array}{r}19070_{41 / 2}-61055_{41 / 2}^{\circ} \\
a^{4} \mathrm{~F}_{31 / 2}-55392^{\circ}{ }^{\circ 1 / 2} \\
a^{4} \mathrm{D}_{21 / 2}-56932_{11 / 2}^{\circ} \\
a^{4} \mathrm{G}_{51 / 2}-59399_{41 / 2}^{\circ} \\
23046_{31 / 2}-65003_{41 / 2}^{\circ}\end{array}$ & \\
\hline $\begin{array}{l}2383.500 \\
2383.884 \\
2384.033 \\
2385.253 \\
2385.335\end{array}$ & $\begin{array}{l}2 \\
1- \\
3 \\
7 \\
5\end{array}$ & $\begin{array}{r}3 \\
1 \\
6 \\
15 \\
7\end{array}$ & $\begin{array}{l}41942.32 \\
41935.57 \\
41932.95 \\
41911.48 \\
41910.04\end{array}$ & $\begin{array}{r}-0.12 \\
-0.03 \\
-0.01 \\
+0.09 \\
0.00\end{array}$ & $\begin{array}{r}a^{4} \mathrm{D}_{31 / 2}-57089_{41 / 2}^{\circ} \\
20780_{41 / 2}-62715_{41 / 2}^{\circ} \\
18000_{31 / 2}-59933_{31 / 2}^{\circ} \\
a^{4} \mathrm{~F}_{41 / 2}-56768_{31 / 2}^{\circ} \\
a^{4} \mathrm{D}_{11 / 2}-56544^{\circ} 2^{11 / 2}\end{array}$ & \\
\hline $\begin{array}{l}2385.500 \\
2386.447 \\
2387.708 \\
2388.536 \\
2388.798\end{array}$ & $\begin{array}{l}8 \\
2 \\
2 \\
2 \\
2\end{array}$ & $\begin{array}{r}12 \\
9 \\
5 \\
8 \\
8\end{array}$ & $\begin{array}{l}41907.14 \\
41890.51 \\
41868.39 \\
41853.88 \\
41849.28\end{array}$ & $\begin{array}{l}-0.03 \\
+0.14 \\
-0.05 \\
+0.12 \\
-0.14\end{array}$ & $\begin{array}{r}a^{4} \mathrm{D}_{21 / 2}-5687_{421 / 2}{ }^{\circ} \\
22139_{21 / 2}-64030_{21 / 2}^{\circ} \\
18000_{31 / 2}-59869_{31 / 2}^{\circ} \\
26158_{41 / 2}-68012_{51 / 2}^{\circ} \\
23046_{31 / 2}-64896_{31 / 2}^{\circ}\end{array}$ & $\begin{array}{l}6 \\
6\end{array}$ \\
\hline $\begin{array}{l}2389.541 \\
2390.371 \\
2390.890 \\
2391.218 \\
2391.574\end{array}$ & $\begin{array}{r}25 \\
4 \\
1 \\
2\end{array}$ & $\begin{array}{r}1 \\
75 \\
30 \\
3 \\
3\end{array}$ & $\begin{array}{l}41836.28 \\
41821.75 \\
41812.68 \\
41806.94 \\
41800.71\end{array}$ & $\begin{array}{r}+0.02 \\
+0.08 \\
+0.24 \\
0.00 \\
-0.08\end{array}$ & $\begin{array}{r}22194_{31 / 2}-64030_{21 / 2}^{\circ} \\
a^{6} \mathrm{~S}_{21 / 2}-49242_{21 / 2}^{\circ} \\
a^{4} \mathrm{~F}_{21 / 2}-53113_{21 / 2}^{\circ} \\
22503_{11 / 2}-64310_{21 / 2}^{\circ} \\
a^{4} \mathrm{D}_{21 / 2}-56768^{\circ}{ }^{1} 1 / 2\end{array}$ & \\
\hline
\end{tabular}


TABle 3. Classified lines of W II-Continued

\begin{tabular}{|c|c|c|c|c|c|c|}
\hline \multirow{2}{*}{$\begin{array}{c}\text { Wavelength } \\
(\AA)\end{array}$} & \multicolumn{2}{|c|}{ Intensity } & \multicolumn{2}{|c|}{ Wave number $\left(\mathrm{cm}^{-1}\right)$} & \multirow{2}{*}{ Combination } & \multirow{2}{*}{$\begin{array}{c}\text { Zeeman } \\
\text { effect }\end{array}$} \\
\hline & Are & Spark & Observed & $O-C$ & & \\
\hline \multicolumn{7}{|l|}{ Air } \\
\hline $\begin{array}{l}\text { 2391. } 717 \\
\text { 2392. } 932 \\
\text { 2393. } 171 \\
\text { 2393. } 768 \\
\text { 2394. } 162\end{array}$ & $\begin{array}{r}4 \\
20 \\
4 \\
1 \\
7\end{array}$ & $\begin{array}{r}8 \\
60 \\
10 \\
3 \\
25\end{array}$ & $\begin{array}{l}41798.22 \\
41777.00 \\
41772.83 \\
41762.41 \\
41755.54\end{array}$ & $\begin{array}{l}-0.05 \\
-0.11 \\
+0.07 \\
-0.18 \\
+0.02\end{array}$ & $\begin{array}{c}19442_{61 / 2}-61240_{51 / 2}^{\circ} \\
a^{6} \mathrm{D}_{31 / 2}-z^{6} \mathrm{~F}^{\circ}{ }^{\circ} 1 / 2 \\
a^{4} \mathrm{G}_{21 / 2}-58007^{\circ}{ }^{\circ} 1 / 2 \\
a^{4} \mathrm{P}_{11 / 2}-52355_{01 / 2}^{\circ} \\
a^{4} \mathrm{~F}_{41 / 2}-56612_{31 / 2}^{\circ}\end{array}$ & res \\
\hline $\begin{array}{l}\text { 2394. } 444 \\
\text { 2394. } 636 \\
\text { 2395. } 036 \\
\text { 2395. } 104 \\
\text { 2395. } 384\end{array}$ & $\begin{array}{r}12 \\
1 \\
1 \\
9 \\
\end{array}$ & $\begin{array}{r}7 \\
7 \\
5 \\
10 \\
1\end{array}$ & $\begin{array}{l}41750.62 \\
41747.27 \\
41740.30 \\
41739.12 \\
41734.24\end{array}$ & $\begin{array}{l}+0.28 \\
-0.04 \\
+0.17 \\
+0.01 \\
+0.01\end{array}$ & $\begin{array}{r}a^{4} \mathrm{~F}_{31 / 2}-55162_{21 / 2}^{\circ} \\
a^{4} \mathrm{G}_{31 / 2}-58336_{21 / 2}^{\circ}-63880^{\circ}{ }^{\circ 1 / 2} \\
22139_{21 / 2}-631_{11 / 2} \\
a^{6} \mathrm{D}_{21 / 2}-44911_{51 / 2}^{\circ} \\
23234_{41 / 2}-64969^{\circ}\end{array}$ & \\
\hline 2395. 664 & 1 & 3 & 41729. 36 & -0.04 & $23955_{51 / 2}-65684^{51 / 2}$ & \\
\hline 2395. 730 & 5 & 8 & 41728. 21 & $\begin{array}{l}+0.01 \\
+0.24\end{array}$ & $\begin{array}{l}a^{4} \mathrm{P}_{21 / 2}-55162_{21 / 2} \\
a^{4} \mathrm{D}_{31}-568 \%\end{array}$ & \\
\hline $\begin{array}{l}2396.221 \\
2397.097 \\
2397.997\end{array}$ & 7 & $\begin{array}{r}10 \\
200 \\
15\end{array}$ & $\begin{array}{l}\text { 41719. } 66 \\
41704.42 \\
41688.77\end{array}$ & $\begin{array}{l}+0.24 \\
-0.03 \\
-0.24 \\
-0.14\end{array}$ & $\begin{array}{c}a^{4} \mathrm{~F}_{11 / 2}-50430_{11 / 2}^{\circ} \\
a^{6} \mathrm{D}_{21 / 2}-z^{6} \mathrm{~F}_{31 / 2}^{\circ} \\
19637_{21 / 2}-61326^{\circ}{ }^{\circ}{ }^{11 / 2}\end{array}$ & $\begin{array}{l}\text { res } \\
\text { res }\end{array}$ \\
\hline $\begin{array}{l}\text { 2398. } 149 \\
2399.332 \\
2399.574 \\
2400.358 \\
2400.519\end{array}$ & $\begin{array}{l}4 \\
1 \\
1 \\
5\end{array}$ & $\begin{array}{r}7 \\
12 \\
4 \\
6 \\
8\end{array}$ & $\begin{array}{l}41686.12 \\
41665.57 \\
41661.37 \\
41647.76 \\
41644.97\end{array}$ & $\begin{array}{l}+0.10 \\
+0.02 \\
+0.02 \\
-0.47 \\
+0.05\end{array}$ & $\begin{array}{rr}22194_{31 / 2} & 63880_{21 / 2}^{\circ} \\
18990_{11 / 2} & 60656_{21 / 2}^{\circ} \\
23234_{41 / 2} & 64896_{31 / 2}^{\circ} \\
22139_{21 / 2} & 63788_{31 / 2}^{\circ} \\
a^{4} \mathrm{D}_{21 / 2} & 56612_{31 / 2}^{\circ}\end{array}$ & \\
\hline $\begin{array}{l}\text { 2400. } 866 \\
\text { 2401. } 863 \\
2402.480 \\
2403.074 \\
2403.222\end{array}$ & $\begin{array}{r}4 \\
5 \\
2 \\
5 \\
10\end{array}$ & $\begin{array}{r}2 \\
12 \\
7 \\
10 \\
15\end{array}$ & $\begin{array}{l}41638.95 \\
41621.67 \\
41610.98 \\
41600.69 \\
41598.13\end{array}$ & $\begin{array}{l}-0.13 \\
+0.08 \\
+0.08 \\
+0.03 \\
-0.16\end{array}$ & $\begin{array}{l}a^{6} \mathrm{D}_{31 / 2}-46355_{21 / 2}^{\circ} \\
a^{4} \mathrm{D}_{31 / 2}-56768_{31 / 2}^{\circ} \\
a^{4} \mathrm{~F}_{31 / 2}-55022_{31 / 2}^{\circ} \\
a^{4} \mathrm{~F}_{21 / 2}-52901_{31 / 2}^{\circ} \\
a^{4} \mathrm{P}_{01 / 2}^{\circ}-504300_{11 / 2}^{\circ}\end{array}$ & \\
\hline $\begin{array}{l}\text { 2403. } 455 \\
2403.762 \\
2405.280 \\
2405.631 \\
2406.576\end{array}$ & $\begin{array}{c}4 \\
-? \\
1-\end{array}$ & $\begin{array}{r}10 \\
2 \\
8 \\
1 \\
1\end{array}$ & $\begin{array}{l}\text { 41594. } 10 \\
41588.79 \\
41562.54 \\
41556.48 \\
41540.16\end{array}$ & $\begin{array}{l}-0.02 \\
+0.03 \\
+0.11 \\
+0.06 \\
+0.34\end{array}$ & $\begin{array}{r}22194_{31 / 2}-63788^{\circ}{ }^{\circ} 1 / 2 \\
a^{4} \mathrm{P}_{21 / 2}-5502 \mathcal{2}_{31 / 2}^{\circ} \\
a^{6} \mathrm{~S}_{21 / 2}-48982_{11 / 2}^{\circ} \\
a^{4} \mathrm{~F}_{41 / 2}-56418_{41 / 2}^{\circ} \\
23450_{21 / 2}-64990^{\circ}{ }^{\circ} 1 / 2\end{array}$ & \\
\hline $\begin{array}{l}\text { 2407. } 286 \\
2407.787 \\
\text { 2408. } 282 \\
\text { 2409. } 226 \\
2409.474\end{array}$ & $\begin{array}{l}1 \\
5 \\
12 A \\
5 \\
3\end{array}$ & $\begin{array}{r}6 \\
12 \\
15 \\
20 \\
18\end{array}$ & $\begin{array}{l}41527.92 \\
41519.27 \\
41510.73 \\
41494.48 \\
41490.21\end{array}$ & $\begin{array}{l}+0.64 \\
+0.04 \\
-0.13 \\
-0.02\end{array}$ & $\begin{array}{r}22503_{11 / 2}-64030_{21 / 2}^{\circ}-56376_{51 / 2}^{\circ} \\
a^{4} \mathrm{~F}_{41 / 2}-561550_{31 / 2}^{\circ} \\
20039_{31 / 2}-615087_{21 / 2}^{\circ}\end{array}$ & res \\
\hline $\begin{array}{l}2409.827 \\
2410.694 \\
2410.854 \\
2411.287 \\
2411.538\end{array}$ & $\begin{array}{r}- \\
\frac{1}{7} \\
6\end{array}$ & $\begin{array}{r}4 \\
2 \\
1 \\
3 \\
20\end{array}$ & $\begin{array}{l}41484.13 \\
41469.21 \\
41466.46 \\
41459.01 \\
41454.70\end{array}$ & $\begin{array}{l}+0.42 \\
-0.36 \\
+0.18 \\
-0.09 \\
-0.02\end{array}$ & $\begin{array}{r}18990_{11 / 2}-60474_{4112}^{\circ} \\
23046_{31 / 2}-64516_{41 / 2}^{\circ} \\
24804_{31 / 2}-66270_{41 / 2}^{\circ} \\
a^{6} \mathrm{D}_{31 / 2}-46175_{31 / 2}^{\circ}-58991_{51 / 2}^{\circ} \\
a^{4} \mathrm{G}_{51 / 2}^{\circ}-58\end{array}$ & \\
\hline $\begin{array}{l}\text { 2411. } 820 \\
2412.064 \\
2412.76 \\
2414.118 \\
2414.806\end{array}$ & $\begin{array}{r}10 \\
2 \\
5 \\
4 \\
6\end{array}$ & $\begin{array}{r}25 \\
2 \\
8 \\
7 \\
25\end{array}$ & $\begin{array}{l}41449.86 \\
41445.66 \\
41433.72 \\
41410.40 \\
41398.60\end{array}$ & $\begin{array}{l}-0.08 \\
-0.06 \\
-0.06 \\
+0.13 \\
-0.04\end{array}$ & $\begin{array}{r}a^{4} \mathrm{D}_{11 / 2}-56084_{11 / 2}^{\circ} \\
23450_{21 / 2}-64896^{\circ}{ }^{\circ 1 / 2} \\
a^{4} \mathrm{G}_{41 / 2}-57986_{41 / 2}^{\circ} \\
a^{6} \mathrm{~S}_{21 / 2}-48830_{31 / 2}^{\circ}-489_{41 / 2}^{\circ} \\
18000_{31 / 2}-59399^{\circ}\end{array}$ & 4 \\
\hline $\begin{array}{l}2414.888 \\
2416.063 \\
2416.416 \\
2417.430 \\
2419.350\end{array}$ & $\begin{array}{l}3 \\
1 \\
2 \\
8\end{array}$ & $\begin{array}{r}3 \\
3 \\
1 \\
4 \\
35\end{array}$ & $\begin{array}{l}\text { 41397. } 20 \\
41377.06 \\
41371.02 \\
41353.67 \\
41320.85\end{array}$ & $\begin{array}{l}-0.05 \\
+0.02 \\
+0.02 \\
+0.21 \\
+0.05\end{array}$ & $\begin{array}{c}a^{4} \mathrm{G}_{31 / 2}-57986_{41 / 2}^{\circ} \\
22503_{11 / 2}-63880_{21 / 2}^{\circ} \\
23955_{51 / 2}-65326_{51 / 2}^{\circ} \\
19070_{41 / 2}-60424^{\circ} 1_{1 / 2} \\
20039_{31 / 2}-61360_{41 / 2}^{\circ}\end{array}$ & \\
\hline $\begin{array}{l}2419.848 \\
2419.987 \\
2420.990 \\
2421.358 \\
2421.662\end{array}$ & $\begin{array}{r}4 \\
3 \\
12 \\
1 \\
3\end{array}$ & $\begin{array}{r}5 \\
8 \\
35 \\
3 \\
12\end{array}$ & $\begin{array}{l}41312.34 \\
41309.98 \\
41292.86 \\
41286.59 \\
41281.40\end{array}$ & $\begin{array}{l}+0.12 \\
+0.08 \\
+0.21 \\
+0.04 \\
-0.10\end{array}$ & $\begin{array}{r}a^{4} \mathrm{D}_{01 / 2}-54485_{01 / 2}^{\circ} \\
23046_{31 / 2}-64356_{31 / 2}^{\circ} \\
a^{4} \mathrm{~F}_{31 / 2}-547044^{\circ} 1 / 2 \\
20039_{31 / 2}-61326_{31 / 2}^{\circ} \\
23234_{41 / 2}-64516_{41 / 2}^{\circ}\end{array}$ & \\
\hline
\end{tabular}


Table 3. Classified lines of W II-Continued

\begin{tabular}{|c|c|c|c|c|c|c|}
\hline \multirow{2}{*}{$\begin{array}{c}\text { Wavelength } \\
(\AA)\end{array}$} & \multicolumn{2}{|c|}{ Intensity } & \multicolumn{2}{|c|}{ Wave number $\left(\mathrm{cm}^{-1}\right)$} & \multirow{2}{*}{ Combination } & \multirow{2}{*}{$\begin{array}{c}\text { Zeeman } \\
\text { effect }\end{array}$} \\
\hline & Arc & Spark & Observed & $O-C$ & & \\
\hline Air & & & & & & \\
\hline $\begin{array}{l}2421.974 \\
2422.292 \\
2422.529 \\
2422.726 \\
2425.975\end{array}$ & $\begin{array}{l}5 \\
30 A \\
8 \\
1 \\
10 A\end{array}$ & $\begin{array}{r}12 \\
20 \\
25 \\
4 \\
6\end{array}$ & $\begin{array}{l}41276.09 \\
41270.67 \\
41266.63 \\
41263.28 \\
41208.02\end{array}$ & $\begin{array}{l}-0.02 \\
+0.16 \\
+0.01 \\
+0.08 \\
-0.01\end{array}$ & $\begin{array}{r}18000_{31 / 2}-59276_{31 / 2}^{\circ} \\
a^{4} \mathrm{P}_{21 / 2}-54704^{\circ}{ }^{1 / 2} \\
a^{4} \mathrm{D}_{1 / 2}-56413_{41 / 2}^{\circ} \\
23046_{31 / 2}-64310_{21 / 2}^{\circ} \\
19070_{41 / 2}-60278_{41 / 2}^{\circ}\end{array}$ & \\
\hline $\begin{array}{l}2426.482 \\
2426.558 \\
2427.493 \\
2427.807 \\
2428.758\end{array}$ & $\begin{array}{r}1 \\
4 \\
10 \\
4 \\
4\end{array}$ & $\begin{array}{r}4 \\
10 \\
40 \\
6 \\
10\end{array}$ & $\begin{array}{l}41199.42 \\
41198.13 \\
41182.26 \\
41176.93 \\
41160.80\end{array}$ & $\begin{array}{l}+0.06 \\
-0.02 \\
-0.04 \\
+0.15 \\
+0.10\end{array}$ & 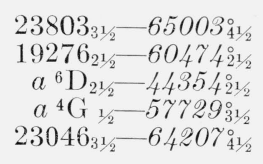 & $\begin{array}{l}6 \\
6 \text { res }\end{array}$ \\
\hline $\begin{array}{l}\text { 2429. } 396 \\
2429.489 \\
2429.528 \\
2429.971 \\
2430.788\end{array}$ & $\begin{array}{l}4 \\
4 \\
3 \\
1 \\
3\end{array}$ & $\begin{array}{r}10 \\
6 \\
8 \\
6 \\
10\end{array}$ & $\begin{array}{l}41149.99 \\
41148.42 \\
41147.76 \\
41140.26 \\
41126.44\end{array}$ & $\begin{array}{l}+0.43 \\
+0.26 \\
+0.14 \\
+0.01 \\
+0.11\end{array}$ & $\begin{array}{rr}26929_{51 / 2}-68078_{61 / 2}^{\circ} \\
19070_{1 / 2}-60218_{51 / 2}^{\circ} \\
19276_{21 / 2}-604243^{\circ 1 / 2} \\
a^{4} \mathrm{G}_{31 / 2}-57729_{31 / 2}^{\circ} \\
22139_{21 / 2}-63266_{31 / 2}^{\circ}\end{array}$ & 6 \\
\hline $\begin{array}{l}\text { 2431. } 373 \\
2431.714 \\
\text { 2432. } 797 \\
2433.139 \\
2433.982\end{array}$ & $\begin{array}{c}5 \\
5 \\
5 \\
25 A\end{array}$ & $\begin{array}{r}18 \\
12 \\
6 \\
8 \\
20\end{array}$ & $\begin{array}{l}41116.54 \\
41110.78 \\
41092.47 \\
41086.70 \\
41072.47\end{array}$ & $\begin{array}{l}+0.06 \\
+0.01 \\
+0.09 \\
+0.09 \\
+0.25\end{array}$ & $\begin{array}{rr}a^{4} \mathrm{D}_{21 / 2} & 56084^{\circ}{ }^{11 / 2} \\
20455_{11 / 2} & 61566_{21 / 2}^{\circ} \\
23803_{1 / 2} & 64896_{31 / 2}^{\circ} \\
a^{4} \mathrm{~F}_{31 / 2} & -54498_{31 / 2}^{\circ} \\
22194_{1 / 2} & 63266^{\circ}{ }^{11 / 2}\end{array}$ & 6 res \\
\hline $\begin{array}{l}2434.254 \\
2434.453 \\
2435.008 \\
2435.445 \\
2437.155\end{array}$ & $\begin{array}{c}10 A \\
5 \\
10 \\
4 \\
1-\end{array}$ & $\begin{array}{r}12 \\
8 \\
50 \\
20 \\
1\end{array}$ & $\begin{array}{l}41067.88 \\
41064.53 \\
41055.17 \\
41047.80 \\
41019.00\end{array}$ & $\begin{array}{r}+0.05 \\
+0.06 \\
+0.06 \\
0.00 \\
-0.13\end{array}$ & $\begin{array}{r}20534_{51 / 2}-61602_{61 / 2}^{\circ} \\
a^{4} \mathrm{P}_{21 / 2}-54498_{31 / 2}^{\circ} \\
20534_{51 / 2}-61589_{51 / 2}^{\circ} \\
23955_{51 / 2}-65003_{41 / 2}^{\circ} \\
196337_{21 / 2}-60656_{21 / 2}^{\circ}\end{array}$ & \\
\hline $\begin{array}{l}2437.332 \\
2437.471 \\
2439.473 \\
2439.808 \\
2439.913\end{array}$ & $\begin{array}{l}3 \\
4 \\
4 \\
2 \\
2\end{array}$ & $\begin{array}{r}6 \\
15 \\
12 \\
7 \\
7\end{array}$ & $\begin{array}{l}41016.02 \\
41013.68 \\
40980.03 \\
40974.40 \\
40972.64\end{array}$ & $\begin{array}{l}-0.04 \\
-0.02 \\
+0.10 \\
+0.20 \\
+0.01\end{array}$ & $\begin{array}{r}20039_{31 / 2}-61055_{41 / 2}^{\circ} \\
23955_{51 / 2}-64969_{51 / 2}^{\circ} \\
19276_{21 / 2}-60256_{31 / 2}^{\circ} \\
a^{4} \mathrm{~F}_{21 / 2}-52275_{31 / 2}^{\circ} \\
23234_{41 / 2}-64207_{41 / 2}^{\circ}\end{array}$ & 6 \\
\hline $\begin{array}{l}\text { 2440. } 432 \\
\text { 2441. } 612 \\
\text { 2441. } 790 \\
2443.858 \\
2446.394\end{array}$ & $\begin{array}{r}10 \\
7\end{array}$ & $\begin{array}{r}20 \\
12 \\
7 \\
2 \\
120\end{array}$ & $\begin{array}{l}40963.93 \\
40944.13 \\
40941.14 \\
40906.51 \\
40864.10\end{array}$ & $\begin{array}{l}+0.11 \\
-0.58 \\
+0.31 \\
+0.05\end{array}$ & $\begin{array}{r}a^{4} \mathrm{D}_{01 / 2}-54137_{11 / 2}^{\circ} \\
a^{4} \mathrm{P}_{21 / 2}-54375_{21 / 2}^{\circ} \\
23450_{21 / 2}^{\circ}-64356_{31 / 2}^{\circ 1} \\
a^{6} \mathrm{~S}_{21 / 2}-48284^{\circ}{ }^{1 / 2}\end{array}$ & 6 \\
\hline $\begin{array}{l}\text { 2446. } 570 \\
2447.255 \\
2447.52 \\
2448.00 \\
2448.237\end{array}$ & $\begin{array}{l}1 \\
4 \\
5 \\
4\end{array}$ & $\begin{array}{r}1 \\
12 \\
1 \\
15 \\
50\end{array}$ & $\begin{array}{l}40861.16 \\
40849.73 \\
40845.3 \\
40837.3 \\
40833.34\end{array}$ & $\begin{array}{l}-0.07 \\
+0.10 \\
-0.2 \\
0.0 \\
+0.04\end{array}$ & $\begin{array}{r}20039_{31 / 2}-60900_{21 / 2}^{\circ} \\
22139_{21 / 2}-62989_{21 / 2}^{\circ} \\
a^{4} \mathrm{P}_{11 / 2}-51438_{21 / 2}^{\circ} \\
19637_{21 / 2}-6044_{421 / 2}^{\circ} \\
23046_{31 / 2}-63880_{21 / 2}^{\circ}\end{array}$ & \\
\hline $\begin{array}{l}\text { 2448. } 662 \\
\text { 2448. } 719 \\
2449.694 \\
2450.324 \\
2451.032\end{array}$ & $\begin{array}{l}4 \\
3 \\
4 \\
2 \\
3\end{array}$ & $\begin{array}{r}8 \\
4 \\
20 \\
7 \\
7\end{array}$ & $\begin{array}{l}40826.26 \\
40825.31 \\
40809.06 \\
40798.57 \\
40786.78\end{array}$ & $\begin{array}{l}+0.07 \\
-0.03 \\
-0.02 \\
+0.11 \\
+0.02\end{array}$ & $\begin{array}{l}20534_{51 / 2}-61360_{41 / 2}^{\circ} \\
18990_{11 / 2}-5.9816_{11 / 2}^{\circ} \\
20780_{41 / 2}-61589_{51 / 2}^{\circ} \\
19070_{41 / 2}-59869_{31 / 2}^{\circ} \\
19637_{21 / 2}-50424^{\circ}{ }^{\circ} 1 / 2\end{array}$ & \\
\hline $\begin{array}{l}\text { 2451. } 468 \\
2451.660 \\
2453.760 \\
2455.506 \\
2455.722\end{array}$ & $\begin{array}{c}3 \\
40 A \\
2\end{array}$ & $\begin{array}{r}50 \\
1 \\
10 \\
10 \\
5\end{array}$ & $\begin{array}{l}40779.53 \\
40776.33 \\
40741.44 \\
40712.48 \\
40708.90\end{array}$ & $\begin{array}{r}+0.11 \\
+0.03 \\
0.04 \\
-0.05 \\
+0.04\end{array}$ & $\begin{array}{c}a^{6} \mathrm{D}_{11 / 2}-42298_{11 / 2}^{\circ} \\
19442_{61 / 2}-60218_{51 / 2}^{\circ} \\
23046_{31 / 2}-63783_{31 / 2}^{\circ} \\
23803_{31 / 2}-64516_{41 / 2}^{\circ} \\
18000_{31 / 2}-58709_{31 / 2}^{\circ}\end{array}$ & \\
\hline $\begin{array}{l}\text { 2455. } 866 \\
2456.072 \\
2457.043 \\
2458.529 \\
2458.564\end{array}$ & $\begin{array}{l}6 \\
4 \\
1 \\
3 \\
5\end{array}$ & $\begin{array}{c}35 \\
8 \\
3 \\
4 ? \\
30\end{array}$ & $\begin{array}{l}40706.51 \\
40703.09 \\
40687.01 \\
40662.42 \\
40661.84\end{array}$ & $\begin{array}{l}+0.05 \\
-0.01 \\
-0.17 \\
+0.09 \\
-0.04\end{array}$ & $\begin{array}{r}20534_{51 / 2}-61240_{51 / 2}^{\circ} \\
a^{4} \mathrm{P}_{21 / 2}-54137_{11 / 2} \\
18000_{31 / 2}-58687^{41 / 2} \\
a^{4} \mathrm{G}_{31 / 2}-57252^{\circ 112} \\
a^{4} \mathrm{P}_{11 / 2}-51254{ }_{11 / 2}^{\circ}\end{array}$ & 6 \\
\hline
\end{tabular}


TABle 3. Classified lines of $\mathrm{W} \mathrm{II}$-Continued

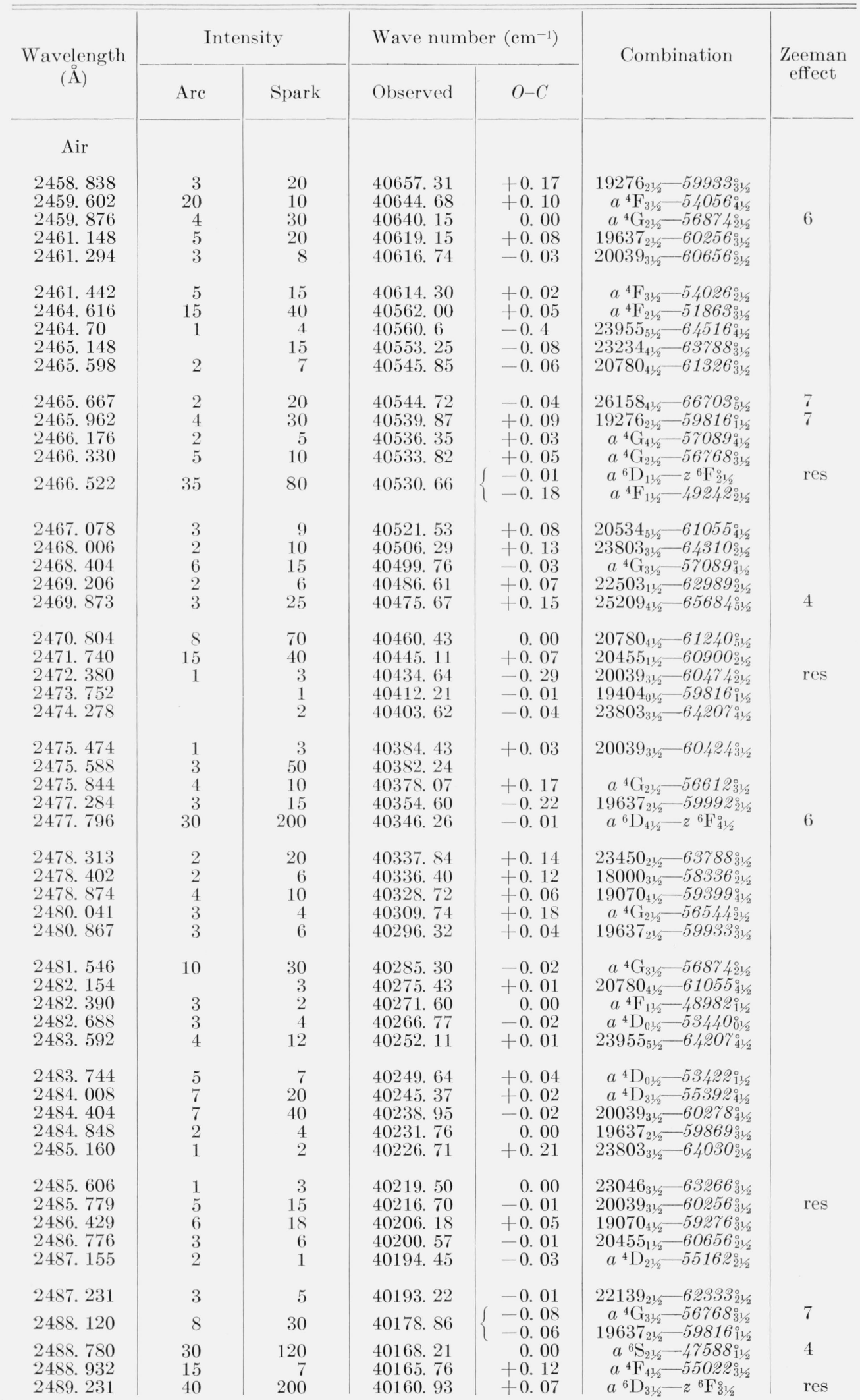


TABLE 3. Classified lines of W II-Continued

\begin{tabular}{|c|c|c|c|c|c|c|}
\hline \multirow{2}{*}{$\begin{array}{l}\text { Wavelength } \\
\text { (A) }\end{array}$} & \multicolumn{2}{|c|}{ Intensity } & \multicolumn{2}{|c|}{ Wave number $\left(\mathrm{cm}^{-1}\right)$} & \multirow{2}{*}{ Combination } & \multirow{2}{*}{$\begin{array}{c}\text { Zeeman } \\
\text { effect }\end{array}$} \\
\hline & Arc & Spark & Observed & $O-C$ & & \\
\hline \multicolumn{7}{|l|}{ Air } \\
\hline $\begin{array}{l}2489.514 \\
2489.900 \\
2490.586 \\
2490.718 \\
2492.252\end{array}$ & $\begin{array}{r}5 \\
5 \\
4 \\
12 \\
4\end{array}$ & $\begin{array}{l}12 \\
10 \\
15 \\
25 \\
14\end{array}$ & $\begin{array}{l}40156.37 \\
40150.14 \\
40139.08 \\
40136.95 \\
40112.25\end{array}$ & $\begin{array}{r}+0.04 \\
-0.06 \\
-0.04 \\
0.00 \\
+0.04\end{array}$ & $\begin{array}{r}a^{4} \mathrm{D}_{01 / 2}-53329_{11 / 2}^{\circ} \\
a^{4} \mathrm{P}_{01 / 2}-48982_{11 / 2}^{\circ} \\
22194_{31 / 2}-62393_{21 / 2}^{\circ} \\
a^{4} \mathrm{~F}_{21 / 2}-51438_{21 / 2}^{\circ} \\
26158_{41 / 2}^{\circ}-66270_{41 / 2}^{\circ}\end{array}$ & \\
\hline $\begin{array}{l}2492.928 \\
2493.54 \\
2494.738 \\
2494.872 \\
2495.522\end{array}$ & $\begin{array}{l}8 \\
3\end{array}$ & $\begin{array}{r}80 \\
7 \\
2 \\
7 \\
10\end{array}$ & $\begin{array}{l}40101.38 \\
40091.54 \\
40072.29 \\
40070.14 \\
40059.70\end{array}$ & $\begin{array}{l}+0.02 \\
-0.01 \\
+0.07 \\
-0.11 \\
+0.10\end{array}$ & $\begin{array}{r}a^{4} \mathrm{~F}_{41 / 2}-54958_{51 / 2}^{\circ} \\
24804_{31 / 2}-64896_{31 / 2}^{\circ} \\
24918_{11 / 2}-64990_{21 / 2}^{\circ} \\
a^{4} \mathrm{D}_{11 / 2}-54704^{\circ}{ }^{\circ} 1 / 2 \\
a^{4} \mathrm{G}_{41 / 2}-56612_{31 / 2}^{\circ}\end{array}$ & \\
\hline $\begin{array}{l}2496.648 \\
2497.480 \\
2498.076 \\
2499.223 \\
2499.330\end{array}$ & $\begin{array}{r}50 \\
35 \\
2 \\
8 \\
2\end{array}$ & $\begin{array}{r}120 \\
75 \\
3 \\
16 \\
4\end{array}$ & $\begin{array}{l}40041.63 \\
40028.29 \\
40018.74 \\
40000.38 \\
39998.67\end{array}$ & $\begin{array}{r}-0.15 \\
+0.03 \\
0.00 \\
+0.09 \\
-0.09\end{array}$ & $\begin{array}{r}a^{6} \mathrm{D}_{31 / 2}-44758_{41 / 2}^{\circ} \\
a^{6} \mathrm{D}_{41 / 2}-46175_{31 / 2}^{\circ} \\
20455_{11 / 2}-60474^{\circ}{ }^{\circ 1 / 2} \\
19276_{21 / 2}-59276_{31 / 2}^{\circ} \\
24991_{11 / 2}-64990_{21 / 2}^{\circ}\end{array}$ & $\begin{array}{l}5 \\
5\end{array}$ \\
\hline $\begin{array}{l}2499.692 \\
2499.934 \\
2500.11 \\
2500.217 \\
2501.020\end{array}$ & $\begin{array}{r}15 \\
3 \\
12 \\
1 \\
1\end{array}$ & $\begin{array}{r}100 \\
4 \\
30 \\
3 \\
10\end{array}$ & $\begin{array}{l}39992.88 \\
39989.00 \\
39986.19 \\
39984.48 \\
39971.64\end{array}$ & $\begin{array}{r}-0.02 \\
+0.12 \\
-0.03 \\
+0.12 \\
-0.05\end{array}$ & $\begin{array}{r}a^{6} \mathrm{~S}_{21 / 2}-47413_{21 / 2}^{\circ} \\
a^{4} \mathrm{P}_{21 / 2}-53422_{11 / 2}^{\circ} \\
18000_{31 / 2}-57986_{41 / 2}^{\circ} \\
23803_{31 / 2}^{\circ}-63788_{31 / 2}^{\circ} \\
25169_{11 / 2}^{\circ}-65141_{21 / 2}^{\circ}\end{array}$ & \multirow[t]{2}{*}{ res } \\
\hline $\begin{array}{l}\text { 2501. } 877 \\
2502.072 \\
2502.162 \\
2502.836 \\
2505.264\end{array}$ & $\begin{array}{l}2 \\
3 \\
1\end{array}$ & $\begin{array}{c}10 \\
10 \\
4 ? \\
3 \\
1\end{array}$ & $\begin{array}{l}39957.95 \\
39954.84 \\
39953.40 \\
39942.64 \\
39903.93\end{array}$ & $\begin{array}{l}-0.06 \\
+0.11 \\
+0.08 \\
-0.16 \\
-0.04\end{array}$ & $\begin{array}{r}a^{4} \mathrm{~F}_{31 / 2}-53369_{41 / 2}^{\circ} \\
a^{4} \mathrm{G}_{31 / 2}-565444^{\circ 1 / 2} \\
a^{4} \mathrm{~F}_{21 / 2}-512544_{11 / 2}^{\circ} \\
23046_{31 / 2}-62989_{21 / 2}^{\circ} \\
a^{4} \mathrm{P}_{21 / 2}-53338_{31 / 2}^{\circ}\end{array}$ & \\
\hline $\begin{array}{l}2505.790 \\
2506.048 \\
2507.994 \\
2508.274 \\
2508.582\end{array}$ & $\begin{array}{r}3 \\
12 \\
2 \\
1\end{array}$ & $\begin{array}{r}5 \\
80 \\
30 \\
3 \\
3\end{array}$ & $\begin{array}{l}39895.56 \\
39891.45 \\
39860.50 \\
39856.05 \\
39851.16\end{array}$ & $\begin{array}{r}-0.05 \\
+0.15 \\
0.00 \\
+0.05 \\
-0.08\end{array}$ & $\begin{array}{r}a{ }^{4} \mathrm{P}_{21 / 2}-53829_{11 / 2}^{\circ} \\
28187_{61 / 2}-68078_{61 / 2}^{\circ} \\
a^{4} \mathrm{G}_{41 / 2}-56413_{41 / 2}^{\circ} \\
18000_{31 / 2}^{\circ}-57856_{21 / 2}^{\circ} \\
a^{4} \mathrm{D}_{11 / 2}-54485_{01 / 2}^{\circ}\end{array}$ & $\begin{array}{l}6 \\
6\end{array}$ \\
\hline $\begin{array}{l}2508.690 \\
2509.386 \\
2509.955 \\
2510.246 \\
2510.348\end{array}$ & $\begin{array}{l}-? \\
2 \\
6 \\
-?\end{array}$ & $\begin{array}{r}4 \\
3 \\
40 \\
3 \\
2\end{array}$ & $\begin{array}{l}39849.44 \\
39838.39 \\
39829.35 \\
39824.74 \\
39823.12\end{array}$ & $\begin{array}{l}-0.02 \\
-0.04 \\
-0.05 \\
-0.16 \\
-0.19\end{array}$ & $\begin{array}{r}a^{4} \mathrm{G}_{21 / 2}-56084{ }^{\circ} 1 / 2 \\
a^{4} \mathrm{P}_{11 / 2}-50430_{11 / 2}^{\circ} \\
20039_{31 / 2}^{\circ}-59869^{\circ}{ }^{\circ} 1 / 2 \\
28187_{61 / 2}-68012_{51 / 2}^{\circ} \\
a^{4} \mathrm{G}_{41 / 2}-56376_{51 / 2}^{\circ}\end{array}$ & 7 \\
\hline $\begin{array}{l}2510.482 \\
2510.799 \\
2512.186 \\
2513.435 \\
2514.358\end{array}$ & $\begin{array}{l}8 \\
2 \\
1 \\
2\end{array}$ & $\begin{array}{r}80 \\
3 \\
4 \\
30 \\
4\end{array}$ & $\begin{array}{l}39821.00 \\
39815.97 \\
39793.99 \\
39774.22 \\
39759.62\end{array}$ & $\begin{array}{l}-0.06 \\
+0.17 \\
+0.07 \\
+0.06 \\
+0.11\end{array}$ & $\begin{array}{r}19070_{41 / 2}-58891_{51 / 2}^{\circ} \\
23450_{21 / 2}-63266_{31 / 2}^{\circ} \\
25209_{41 / 2}-65003_{41 / 2}^{\circ} \\
26929_{51 / 2}^{\circ}-66703_{51 / 2}^{\circ} \\
a^{6} \mathrm{~S}_{21 / 2}-47179_{11 / 2}^{\circ}\end{array}$ & 6 \\
\hline 2514.526 & 3 & 20 & 39756. 96 & -0.02 & $18990_{11 / 2}-58747_{11 / 2}^{\circ}$ & \multirow[t]{3}{*}{ res } \\
\hline 2515. 324 & 3 & 30 & 39744.35 & $\begin{array}{l}+0.18 \\
-0.01\end{array}$ & $\begin{array}{c}a^{4} \mathrm{~F}_{21 / 2}-51045_{31 / 2}^{\circ} \\
20534_{51-6027}^{\circ}-60\end{array}$ & \\
\hline $\begin{array}{l}2515.508 \\
2515.806 \\
2516.138\end{array}$ & $\begin{array}{l}5 \\
2\end{array}$ & $\begin{array}{r}8 \\
10 \\
10\end{array}$ & $\begin{array}{l}39741.44 \\
39736.73 \\
39731.49\end{array}$ & $\begin{array}{l}-0.01 \\
-0.06 \\
-0.14\end{array}$ & $\begin{array}{r}a^{4} \mathrm{D}_{11 / 2}-54375_{21 / 2}^{\circ} \\
a^{4} \mathrm{D}_{21 / 2}-547044^{\circ} 1 \frac{1}{2} \\
23234_{41 / 2}-62966_{51 / 2}^{\circ}\end{array}$ & \\
\hline $\begin{array}{l}2516.284 \\
2517.406 \\
2518.144 \\
2518.973 \\
2519.126\end{array}$ & $\begin{array}{l}1 \\
1 \\
6 \\
4\end{array}$ & $\begin{array}{r}3 \\
25 \\
50 \\
4 \\
7\end{array}$ & $\begin{array}{l}39729.18 \\
39711.48 \\
39699.84 \\
39686.78 \\
39684.37\end{array}$ & $\begin{array}{l}-0.04 \\
-0.22 \\
+0.03 \\
-0.16 \\
-0.12\end{array}$ & $\begin{array}{r}18000_{31 / 2}-57729_{31 / 2}^{\circ} \\
24804_{31 / 2}-64516_{41 / 2}^{\circ} \\
a^{4} \mathrm{P}_{11 / 2}-50292_{21 / 2}^{\circ} \\
25209_{41 / 2}-64896_{31 / 2}^{\circ} \\
20534_{51 / 2}^{\circ}-60218_{51 / 2}^{\circ}\end{array}$ & 5 \\
\hline
\end{tabular}


TABLE 3. Classified lines of $\mathrm{W} \mathrm{II}$-Continued

\begin{tabular}{|c|c|c|c|c|c|c|}
\hline \multirow{2}{*}{$\begin{array}{c}\text { Wavelength } \\
(\AA)\end{array}$} & \multicolumn{2}{|c|}{ Intensity } & \multicolumn{2}{|c|}{ Wave number $\left(\mathrm{cm}^{-1}\right)$} & \multirow{2}{*}{ Combination } & \multirow{2}{*}{$\begin{array}{c}\text { Zeeman } \\
\text { effect }\end{array}$} \\
\hline & Arc & Spark & Observed & $O-C$ & & \\
\hline \multicolumn{7}{|l|}{ Air } \\
\hline $\begin{array}{l}2519.444 \\
2520.10 \\
2521.156 \\
2521.686 \\
2521.853\end{array}$ & $\begin{array}{l}8 \\
1 \\
3 \\
3 A \\
3\end{array}$ & $\begin{array}{r}30 \\
3 \\
10 \\
3 \\
3\end{array}$ & $\begin{array}{l}\text { 39679. } 36 \\
39669.0 \\
39652.42 \\
39644.09 \\
39641.46\end{array}$ & $\begin{array}{l}-0.06 \\
-0.2 \\
-0.02 \\
+0.33 \\
+0.11\end{array}$ & $\begin{array}{r}a^{4} \mathrm{P}_{21 / 2}-53113_{21 / 2}^{\circ} \\
23046_{31 / 2}-62715_{41 / 2}^{\circ} \\
a^{4} \mathrm{G}_{51 / 2}-57089^{\circ 11 / 2} \\
20780_{41 / 2}^{\circ}-604243^{\circ 1 / 2} \\
a^{4} \mathrm{~F}_{41 / 2}-54498^{\circ} 1 \frac{12}{2}\end{array}$ & res \\
\hline $\begin{array}{l}2522.039 \\
2522.270 \\
2526.208 \\
2527.200 \\
2527.552\end{array}$ & $\begin{array}{r}30 \\
3 \\
4 \\
2 \\
2\end{array}$ & $\begin{array}{r}60 \\
8 \\
10 \\
15 \\
10\end{array}$ & $\begin{array}{l}39638.54 \\
39634.91 \\
39573.12 \\
39557.59 \\
39552.08\end{array}$ & $\begin{array}{r}+0.04 \\
+0.21 \\
-0.10 \\
0.00 \\
+0.05\end{array}$ & $\begin{array}{r}a^{6} \mathrm{D}_{31 / 2}-4435421 \frac{1}{2} \\
28377^{11 / 2}-68012^{\circ 1 / 2} \\
a^{4} \mathrm{~F}_{11 / 2}-4828421 / 2 \\
a^{4} \mathrm{D}_{31 / 2}-547042^{11 / 2} \\
24804_{31 / 2}-64356_{31 / 2}^{\circ}\end{array}$ & res \\
\hline $\begin{array}{l}2528.560 \\
2528.913 \\
2529.209 \\
2530.54 \\
2530.70\end{array}$ & $\begin{array}{c}-? \\
5 \\
2 \\
1 \\
10\end{array}$ & $\begin{array}{r}8 \\
20 \\
8 \\
4 \\
8\end{array}$ & $\begin{array}{l}39536.32 \\
39530.80 \\
39526.17 \\
39505.38 \\
39502.88\end{array}$ & $\begin{array}{l}+0.05 \\
+0.05 \\
+0.11 \\
+0.05 \\
+0.04\end{array}$ & $\begin{array}{r}20455_{11 / 2}-59992_{21 / 2}^{\circ} \\
a^{4} \mathrm{D}_{21 / 2}-54498_{31 / 2}^{\circ} \\
26158_{41 / 2}-65684^{\circ} 5_{1 / 2} \\
24804_{31 / 2}-64310_{21 / 2}^{\circ} \\
a^{4} \mathrm{D}_{11 / 2}-54137_{11 / 2}^{\circ}\end{array}$ & res \\
\hline $\begin{array}{l}\text { 2530. } 986 \\
\text { 2532. } 085 \\
2532.414 \\
2532.711 \\
2532.842\end{array}$ & $\begin{array}{l}5 \\
1 \\
4 \\
2 \\
2\end{array}$ & $\begin{array}{r}50 \\
1 \\
7 \\
5 \\
3\end{array}$ & $\begin{array}{l}39498.42 \\
39481.28 \\
39476.15 \\
39471.52 \\
39469.48\end{array}$ & $\begin{array}{l}+0.09 \\
+0.17 \\
+0.08 \\
+0.10 \\
+0.08\end{array}$ & $\begin{array}{l}20780_{41 / 2}-60278_{41 / 2}^{\circ} \\
23234_{41 / 2}-62715_{41 / 2}^{\circ} \\
20780_{41 / 2}-60256_{31 / 2}^{\circ} \\
19276_{21 / 2}-58747_{11 / 2}^{\circ} \\
25672_{21 / 2}-65141_{21 / 2}^{\circ}\end{array}$ & \\
\hline $\begin{array}{l}\text { 2532. } 960 \\
2533.278 \\
2534.140 \\
2534.829 \\
2535.178\end{array}$ & $\begin{array}{l}6 \\
2 \\
5 \\
5\end{array}$ & $\begin{array}{r}15 \\
7 \\
30 \\
50 \\
3\end{array}$ & $\begin{array}{l}\text { 39467. } 64 \\
39462.69 \\
39449.27 \\
39438.55 \\
39433.12\end{array}$ & $\begin{array}{r}0.00 \\
+0.23 \\
+0.07 \\
+0.09 \\
+0.08\end{array}$ & $\begin{array}{r}a^{4} \mathrm{P}_{21 / 2}-52901_{31 / 2}^{\circ} \\
23803_{31 / 2}^{\circ}-63266_{31 / 2}^{\circ} \\
19442_{61 / 2}^{\circ}-58891_{51 / 2}^{\circ} \\
20780_{41 / 2}-60218_{51 / 2}^{\circ} \\
19276_{21 / 2}-58709_{31 / 2}^{\circ}\end{array}$ & $\begin{array}{l}7 \\
7\end{array}$ \\
\hline $\begin{array}{l}2535.573 \\
2536.000 \\
2536.622 \\
2536.789 \\
2537.133\end{array}$ & $\begin{array}{l}1 \\
6 \\
2 \\
1 \\
1\end{array}$ & $\begin{array}{r}10 \\
40 \\
25 \\
1 \\
30\end{array}$ & $\begin{array}{l}39426.98 \\
39420.34 \\
39410.67 \\
39408.07 \\
39402.73\end{array}$ & $\begin{array}{r}+0.25 \\
0.00 \\
+0.04 \\
+0.07 \\
-0.10\end{array}$ & $\begin{array}{r}22139_{21 / 2}-61566_{21 / 2}^{\circ} \\
a^{4} \mathrm{D}_{01 / 2}-52593_{01 / 2}^{\circ} \\
22139_{21 / 2}-61550_{31 / 2}^{\circ} \\
a^{4} \mathrm{D}_{21 / 2}-54375_{21 / 2}^{\circ} \\
24804_{31 / 2}^{\circ}-64207_{41 / 2}^{\circ}\end{array}$ & 4 \\
\hline $\begin{array}{l}\text { 2539. } 313 \\
2539.869 \\
2539.919 \\
2540.101 \\
2540.431\end{array}$ & $\begin{array}{l}7 \\
1 \\
3 \\
1 \\
1\end{array}$ & $\begin{array}{r}35 \\
8 \\
20 \\
4\end{array}$ & $\begin{array}{l}39368.91 \\
39360.29 \\
39359.52 \\
39356.70 \\
39351.58\end{array}$ & $\begin{array}{l}+0.01 \\
-0.08 \\
-0.08 \\
+0.18 \\
+0.03\end{array}$ & $\begin{array}{r}a^{4} \mathrm{P}_{21 / 2}-52803_{11 / 2}^{\circ} \\
20455_{11 / 2}-59816_{11 / 2}^{\circ} \\
20039_{31 / 2}-59399_{41 / 2}^{\circ} \\
22194_{31 / 2}-61550_{31 / 2}^{\circ} \\
a^{4} \mathrm{D}_{31 / 2}-54498_{31 / 2}^{\circ}\end{array}$ & 4 \\
\hline $\begin{array}{l}2540.807 \\
2540.925 \\
2541.063 \\
2542.598 \\
2543.308\end{array}$ & $\begin{array}{c}10 A \\
6 \\
1 \\
2\end{array}$ & $\begin{array}{r}2 \\
4 \\
20 \\
30 \\
25\end{array}$ & $\begin{array}{l}\text { 39345. } 76 \\
39343.93 \\
39341.80 \\
39318.05 \\
39307.07\end{array}$ & $\begin{array}{l}-0.26 \\
+0.07 \\
+0.19 \\
-0.11 \\
-0.02\end{array}$ & $\begin{array}{l}18990_{11 / 2}-58336_{21 / 2}^{\circ} \\
19404_{01 / 2}-58747_{11 / 2} \\
26929_{51 / 2}-66270_{41 / 2}^{\circ} \\
25672_{21 / 2}-64990_{21 / 2}^{\circ} \\
25209_{41 / 2}^{\circ}-64516_{41 / 2}^{\circ}\end{array}$ & $\begin{array}{l}6 \\
6\end{array}$ \\
\hline $\begin{array}{l}2544.658 \\
2546.283 \\
2546.790 \\
2546.912 \\
2547.838\end{array}$ & $\begin{array}{l}3 \\
1 \\
1\end{array}$ & $\begin{array}{r}1 \\
30 \\
15 \\
2 \\
5\end{array}$ & $\begin{array}{l}39286.22 \\
39261.15 \\
39253.33 \\
39251.45 \\
39237.19\end{array}$ & $\begin{array}{l}-0.18 \\
+0.16 \\
+0.15 \\
+0.12\end{array}$ & $\begin{array}{c}23046_{31 / 2}-62333_{21 / 2}^{\circ} \\
a^{4} \mathrm{G}_{21 / 2}-55488_{11 / 2}^{\circ} \\
18000_{31 / 2}^{\circ}-57252_{21 / 2}^{\circ} \\
20039_{31 / 2}^{\circ}-59276_{31 / 2}^{\circ}\end{array}$ & res \\
\hline $\begin{array}{l}2548.378 \\
2548.690 \\
2549.096 \\
2550.10 \\
2550.295\end{array}$ & $\begin{array}{l}6 \\
1 \\
5 \\
5\end{array}$ & $\begin{array}{r}20 \\
6 \\
15 \\
4 \\
8\end{array}$ & $\begin{array}{l}39228.88 \\
39224.08 \\
39217.83 \\
39202.38 \\
39199.39\end{array}$ & $\begin{array}{l}+0.08 \\
+0.02 \\
+0.08 \\
+0.21 \\
+0.07\end{array}$ & $\begin{array}{r}a^{4} \mathrm{D}_{31 / 2}-54375_{21 / 2}^{\circ} \\
25672_{21 / 2}^{\circ}-64896_{31 / 2}^{\circ} \\
a^{6} \mathrm{D}_{21 / 2}-42390_{31 / 2}^{\circ} \\
23235_{41 / 2}^{\circ}-62437_{41 / 2}^{\circ} \\
a^{4} \mathrm{~F}_{41 / 2}-54056_{41 / 2}^{\circ}\end{array}$ & res \\
\hline $\begin{array}{l}2551.157 \\
2551.450 \\
2552.249 \\
2552.362 \\
2553.168\end{array}$ & $\begin{array}{c}1 \\
5 \\
4 \\
70 A\end{array}$ & $\begin{array}{r}10 \\
15 \\
5 \\
40 \\
50\end{array}$ & $\begin{array}{l}39186.15 \\
39181.65 \\
39169.38 \\
39167.65 \\
39155.29\end{array}$ & $\begin{array}{r}-0.17 \\
-0.08 \\
0.00 \\
-0.01 \\
+0.10\end{array}$ & $\begin{array}{r}22139_{21 / 2}-61326_{31 / 2}^{\circ} \\
a^{4} \mathrm{D}_{01 / 2}-52355_{01 / 2}^{\circ} \\
a^{4} \mathrm{D}_{21 / 2}-54137_{11 / 2}^{\circ} \\
26158_{41 / 2}-65326_{51 / 2}^{\circ} \\
a^{4} \mathrm{~F}_{31 / 2}-5256_{41 / 2}^{\circ}\end{array}$ & $\begin{array}{l}\text { res } \\
7 \\
\text { res }\end{array}$ \\
\hline
\end{tabular}


Table 3. Classified lines of W $\mathrm{II}$-Continued

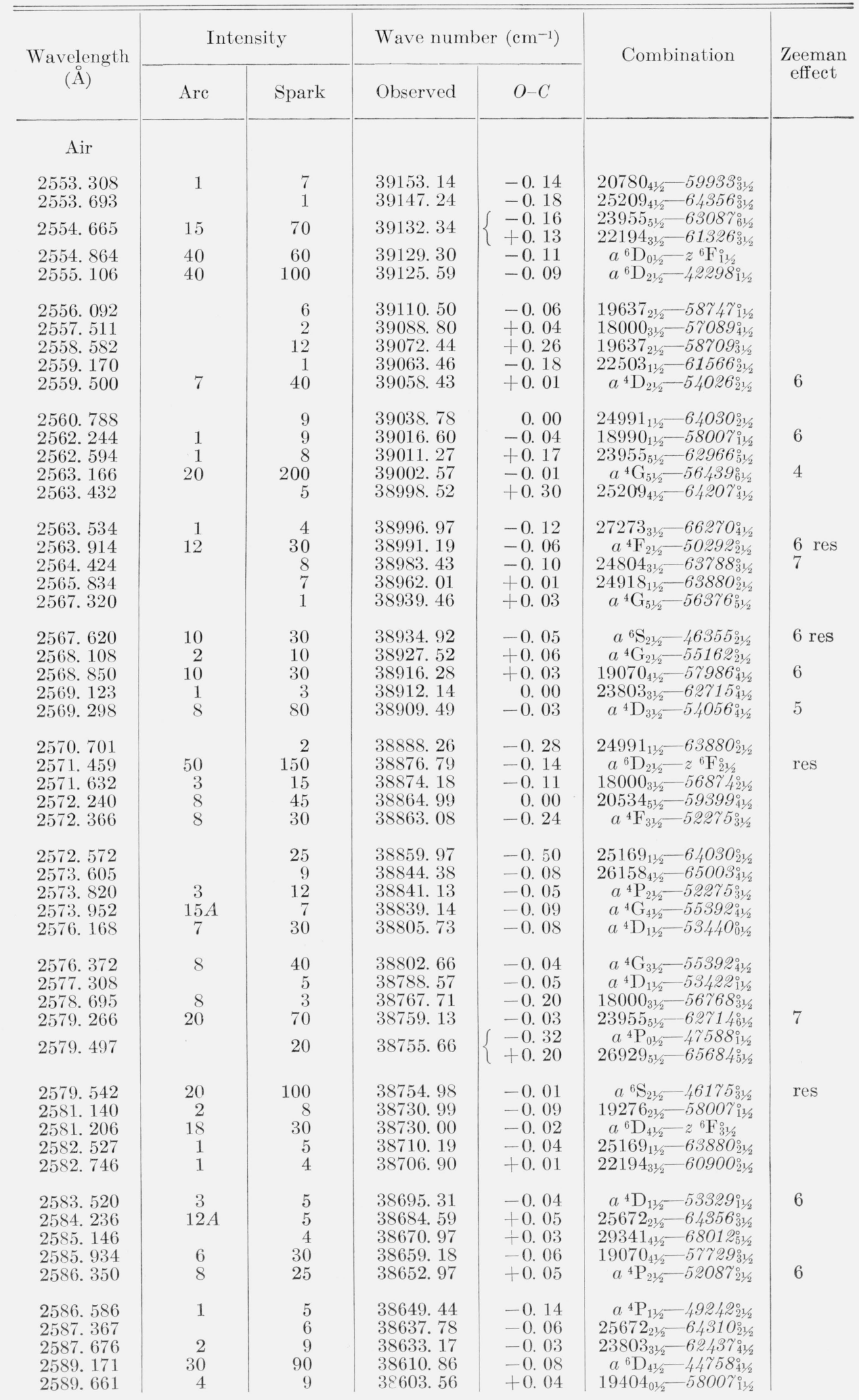


Table 3. Classified lines of $\mathrm{W} \mathrm{II}$-Continued

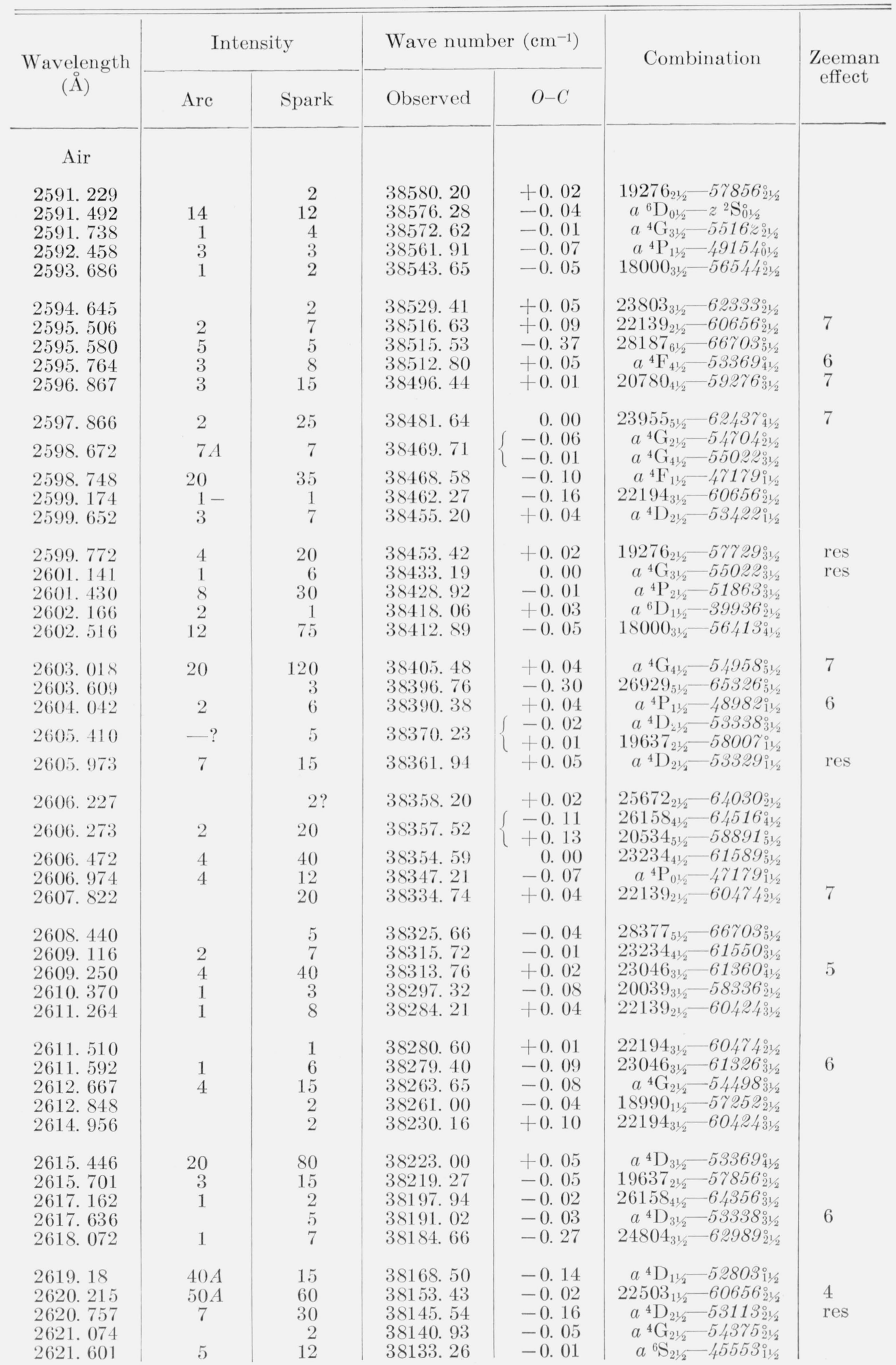


TABle 3. Classified lines of $\mathrm{W} \mathrm{II}$-Continued

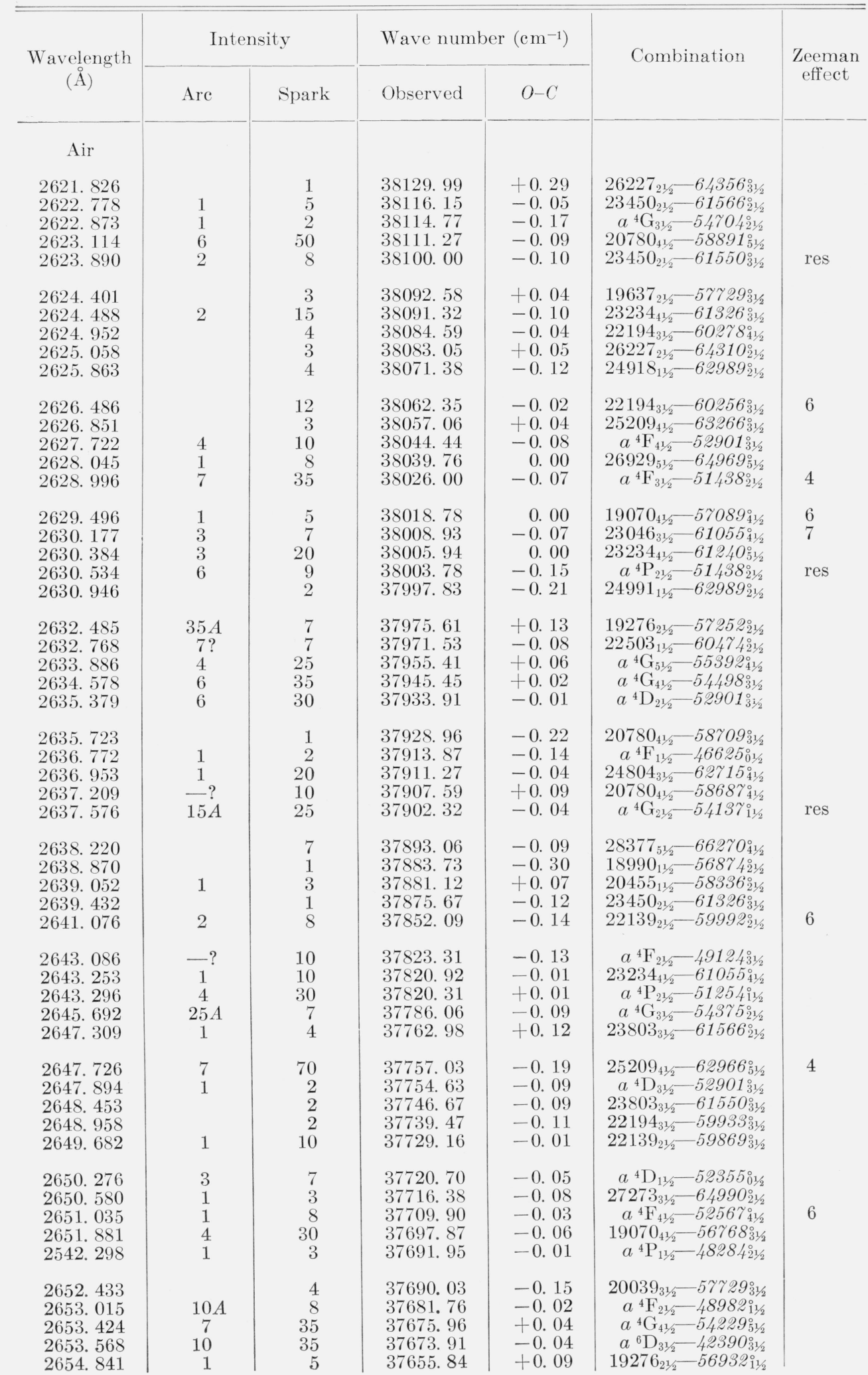


TABle 3. Classified lines of $\mathrm{W} \mathrm{II}$-Continued

\begin{tabular}{|c|c|c|c|c|c|c|}
\hline \multirow{2}{*}{$\begin{array}{c}\text { Wavelength } \\
(\AA)\end{array}$} & \multicolumn{2}{|c|}{ Intensity } & \multicolumn{2}{|c|}{ Wave number $\left(\mathrm{cm}^{-1}\right)$} & \multirow{2}{*}{ Combination } & \multirow{2}{*}{$\begin{array}{c}\text { Zeeman } \\
\text { effect }\end{array}$} \\
\hline & Are & Spark & Observed & $O-C$ & & \\
\hline Air & & & & & & \\
\hline $\begin{array}{l}\text { 2655. } 485 \\
2655.667 \\
2656.027 \\
2656.435 \\
2656.706\end{array}$ & $\begin{array}{r}3 \\
15 \\
-?\end{array}$ & $\begin{array}{r}25 \\
20 \\
10 \\
8 \\
10\end{array}$ & $\begin{array}{l}37646.71 \\
37644.13 \\
27639.03 \\
37633.25 \\
37629.41\end{array}$ & $\begin{array}{l}-0.07 \\
-0.01 \\
-0.06 \\
-0.04 \\
-0.05\end{array}$ & $\begin{array}{r}23955_{51 / 2}-61602_{61 / 2}^{\circ} \\
a{ }^{4} \mathrm{~F}_{11 / 2}-46355_{21 / 2}^{\circ} \\
28631_{31 / 2}-66270_{41 / 2}^{\circ} \\
a{ }^{4} \mathrm{~F}_{31 / 2}-51045_{31 / 2}^{\circ} \\
26158_{41 / 2}-63788_{31 / 2}^{\circ}\end{array}$ & \\
\hline $\begin{array}{l}2657.745 \\
2658.036 \\
2659.171 \\
2659.703 \\
2661.852\end{array}$ & $\begin{array}{c}1 \\
25 \\
1 ? \\
1 \\
1\end{array}$ & $\begin{array}{r}6 \\
100 \\
10 \\
15 \\
20\end{array}$ & $\begin{array}{l}37614.71 \\
37610.59 \\
37594.53 \\
37587.02 \\
37556.67\end{array}$ & $\begin{array}{l}+0.09 \\
-0.04 \\
+0.39 \\
-0.01 \\
-0.03\end{array}$ & $\begin{array}{c}19637_{21 / 2}-57252_{21 / 2}^{\circ} \\
a^{6} \mathrm{D}_{11 / 2}-z^{6} \mathrm{~F}_{11 / 2}^{\circ} \\
25672_{31 / 2}-63266_{31 / 2}^{\circ} \\
26929_{51 / 2}-64516_{41 / 2}^{\circ} \\
23803_{31 / 2}-61360_{41 / 2}^{\circ}\end{array}$ & res \\
\hline $\begin{array}{l}\text { 2662. } 214 \\
\text { 2663. } 874 \\
\text { 2664. } 346 \\
\text { 2665. } 644 \\
\text { 2666. } 086\end{array}$ & $\begin{array}{r}3 \\
2 \\
80 \\
2 \\
1\end{array}$ & $\begin{array}{r}30 \\
15 \\
200 \\
8 \\
40\end{array}$ & $\begin{array}{l}37551.57 \\
37528.17 \\
37521.52 \\
27503.25 \\
37497.03\end{array}$ & $\begin{array}{l}-0.10 \\
-0.02 \\
-0.04 \\
-0.15 \\
-0.17\end{array}$ & $\begin{array}{r}20455_{11 / 2}-58007_{11 / 2}^{\circ} \\
19404_{01 / 2}-56932_{11 / 2}^{\circ} \\
a^{4} \mathrm{G}_{51 / 2}-54958_{51 / 2}^{\circ} \\
a^{4} \mathrm{G}_{41 / 2}-54056_{41 / 2}^{\circ} \\
28187_{61 / 2}-65684^{\circ}{ }^{\circ} 1 / 2\end{array}$ & res \\
\hline $\begin{array}{l}\text { 2666. } 446 \\
\text { 2666. } 493 \\
\text { 2668. } 245 \\
\text { 2668. } 961 \\
\text { 2669. } 250\end{array}$ & $\begin{array}{r}8 \\
20 \\
1 \\
5 \\
10\end{array}$ & $\begin{array}{r}? \\
60 \\
5 \\
5 \\
25\end{array}$ & $\begin{array}{l}37491.97 \\
37491.31 \\
37466.69 \\
37456.64 \\
37452.59\end{array}$ & $\begin{array}{l}-0.12 \\
+0.11 \\
-0.18 \\
-0.11 \\
-0.07\end{array}$ & $\begin{array}{r}19276_{21 / 2}-56768_{31 / 2}^{\circ} \\
a{ }^{6} \mathrm{~S}_{21 / 2}-44911_{11 / 2}^{\circ} \\
a^{4} \mathrm{G}_{31 / 2}-54056_{41 / 2}^{\circ} \\
a^{6} \mathrm{~S}_{21 / 2}-z^{6} \mathrm{~F}_{31 / 2}^{\circ} \\
a^{4} \mathrm{D}_{11 / 2}-52087^{\circ}{ }^{1} 1 / 2\end{array}$ & \\
\hline $\begin{array}{l}2669.371 \\
2670.395 \\
2671.040 \\
2671.580 \\
2672.647\end{array}$ & $\begin{array}{r}5 \\
10 \\
8\end{array}$ & $\begin{array}{r}30 \\
50 \\
2 \\
18 \\
15\end{array}$ & $\begin{array}{l}37450.89 \\
37436.52 \\
37427.49 \\
37419.92 \\
37404.98\end{array}$ & $\begin{array}{l}-0.20 \\
-0.04 \\
-0.38 \\
-0.21 \\
-0.16\end{array}$ & $\begin{array}{r}a^{4} \mathrm{~F}_{31 / 2}-50863^{\circ}{ }^{\circ 1 / 2} \\
a^{4} \mathrm{G}_{31 / 2}-54026_{21 / 2}^{\circ} \\
23046_{31 / 2}-6047_{421 / 2}{ }^{\circ}{ }^{\circ} \\
a^{4} \mathrm{D}_{31 / 2}-52567^{41 / 2} \\
23955_{51 / 2}-61360_{41 / 2}^{\circ}\end{array}$ & \\
\hline $\begin{array}{l}2672.956 \\
2673.608 \\
2674.471 \\
2674.628 \\
2675.734\end{array}$ & $\begin{array}{r}1 \\
20\end{array}$ & $\begin{array}{r}3 \\
60 \\
4 \\
10 \\
20\end{array}$ & $\begin{array}{l}37400.66 \\
37391.54 \\
37379.48 \\
37377.29 \\
37361.84\end{array}$ & $\begin{array}{r}-0.11 \\
-0.13 \\
0.00 \\
-0.05 \\
-0.10\end{array}$ & $\begin{array}{l}20455_{11 / 2}-57856_{21 / 2}^{\circ} \\
18000_{31 / 2}-55392_{41 / 2}^{\circ} \\
30633_{41 / 2}-68012^{\circ} 112 \\
23046_{31 / 2}-60424_{31 / 2}^{\circ} \\
29341_{41 / 2}-66703_{51 / 2}^{\circ}\end{array}$ & res \\
\hline $\begin{array}{l}2677.579 \\
2677.796 \\
2679.638 \\
2679.758 \\
2680.546\end{array}$ & $\begin{array}{r}3 \\
20 \\
20 \\
3\end{array}$ & $\begin{array}{r}6 \\
60 \\
70 \\
18 \\
10\end{array}$ & $\begin{array}{l}\text { 37336. } 10 \\
37333.07 \\
37307.41 \\
37305.74 \\
37294.77\end{array}$ & $\begin{array}{l}-0.12 \\
-0.06 \\
-0.05 \\
-0.03 \\
-0.12\end{array}$ & $\begin{array}{c}19276_{21 / 2}-56612_{31 / 2}^{\circ} \\
a^{6} \mathrm{D}_{31 / 2}-z^{6} \mathrm{~F}_{21 / 2}^{\circ} \\
a^{4} \mathrm{D}_{21 / 2}-5227^{\circ} 5_{31 / 2}^{\circ} \\
19070_{41 / 2}-56376_{51 / 2}^{\circ} \\
19637_{21 / 2}^{\circ}-56932^{\circ 11 / 2}\end{array}$ & \\
\hline $\begin{array}{l}2681.568 \\
2681.730 \\
2683.226 \\
2683.512 \\
2683.632\end{array}$ & $\begin{array}{l}8 \\
1 \\
4\end{array}$ & $\begin{array}{r}2 \\
1 \\
80 \\
35 \\
30\end{array}$ & $\begin{array}{l}37280.55 \\
37278.30 \\
37257.52 \\
37253.56 \\
37251.89\end{array}$ & $\begin{array}{l}+0.05 \\
+0.14 \\
-0.05 \\
-0.07\end{array}$ & $\begin{array}{r}22535_{01 / 2}-59816_{11 / 2}^{\circ} \\
26929_{51 / 2}-64207^{\circ 1 / 2} \\
a^{4} \mathrm{D}_{01 / 2}-50430_{11 / 2}^{\circ} \\
23803_{31 / 2}-61055_{41 / 2}^{\circ}\end{array}$ & \\
\hline $\begin{array}{l}\text { 2684. } 301 \\
2685.068 \\
2685.366 \\
2686.946 \\
2687.000\end{array}$ & $\begin{array}{r}12 \\
12 \\
6 \\
4 \\
5\end{array}$ & $\begin{array}{r}20 \\
25 \\
12 \\
100 \\
70\end{array}$ & $\begin{array}{l}37242.60 \\
37231.96 \\
37227.84 \\
37205.95 \\
37205.20\end{array}$ & $\begin{array}{l}+0.09 \\
+0.05 \\
+0.08 \\
-0.06 \\
-0.06\end{array}$ & $\begin{array}{l}27273_{31 / 2}-64516_{41 / 2}^{\circ} \\
23046_{31 / 2}-60278_{41 / 2}^{\circ} \\
25209_{41 / 2}-62437_{41 / 2}^{\circ} \\
23450_{21 / 2}-60656_{21 / 2}^{\circ} \\
22194_{31 / 2}-59399_{41 / 2}^{\circ}\end{array}$ & res \\
\hline $\begin{array}{l}\text { 2688. } 230 \\
2690.036 \\
2690.153 \\
2690.710 \\
2691.952\end{array}$ & $\begin{array}{c}7 \\
-? \\
2 \\
2\end{array}$ & $\begin{array}{r}35 \\
2 \\
1 \\
35 \\
5\end{array}$ & $\begin{array}{l}37188.17 \\
37163.21 \\
37161.59 \\
37153.90 \\
37136.74\end{array}$ & $\begin{array}{l}+0.03 \\
-0.12 \\
-0.01 \\
-0.10\end{array}$ & $\begin{array}{c}a^{4} \mathrm{G}_{21 / 2}-53422_{11 / 2}^{\circ} \\
25169_{11 / 2}-62333_{21 / 2}^{\circ} \\
18000_{31 / 2}-55162_{21 / 2}^{\circ} \\
22139_{21 / 2}-59276_{31 / 2}^{\circ}\end{array}$ & \\
\hline $\begin{array}{l}\text { 2692. } 358 \\
\text { 2693. } 228 \\
2694.080 \\
\text { 2694. } 382 \\
\text { 2694. } 594\end{array}$ & $\begin{array}{r}20 \\
6\end{array}$ & $\begin{array}{c}18 \\
1 \\
4 ? \\
60 \\
70\end{array}$ & $\begin{array}{l}37131.14 \\
37119.14 \\
37107.40 \\
37103.24 \\
37100.32\end{array}$ & $\begin{array}{l}-0.09 \\
-0.06 \\
-0.16 \\
+0.01 \\
-0.08\end{array}$ & $\begin{array}{r}19637_{21 / 2}-56768_{31 / 2}^{\circ} \\
a^{4} \mathrm{D}_{21 / 2}-52087^{\circ 1 / 2} \\
26158_{41 / 2}-63266_{31 / 2}^{\circ} \\
a^{4} \mathrm{G}_{21 / 2}-53338_{31 / 2}^{\circ} \\
23955_{51 / 2}-61055_{41 / 2}^{\circ}\end{array}$ & \\
\hline
\end{tabular}


TABle 3. Classified lines of $\mathrm{W}$ II-Continued

\begin{tabular}{|c|c|c|c|c|c|c|}
\hline \multirow{2}{*}{$\begin{array}{c}\text { Wavelength } \\
(\AA)\end{array}$} & \multicolumn{2}{|c|}{ Intensity } & \multicolumn{2}{|c|}{ Wave number $\left(\mathrm{cm}^{-1}\right)$} & \multirow{2}{*}{ Combination } & \multirow{2}{*}{$\begin{array}{c}\text { Zeeman } \\
\text { effect }\end{array}$} \\
\hline & Arc & Spark & Observed & $O-C$ & & \\
\hline \multicolumn{7}{|l|}{ Air } \\
\hline $\begin{array}{l}\text { 2694. } 828 \\
2694.994 \\
2695.111 \\
2695.874 \\
2696.914\end{array}$ & $\begin{array}{l}2 \\
6 \\
3 \\
2\end{array}$ & $\begin{array}{r}6 \\
30 \\
1 \\
10 \\
35\end{array}$ & $\begin{array}{l}37097.10 \\
37094.82 \\
37093.21 \\
37082.72 \\
37068.41\end{array}$ & $\begin{array}{l}-0.03 \\
-0.05 \\
-0.13 \\
-0.01\end{array}$ & $\begin{array}{r}23803_{31 / 2}-60900_{21 / 2}^{\circ} \\
a^{4} \mathrm{G}_{21 / 2}-53329_{11 / 2}^{\circ} \\
18990_{11 / 2}-56084_{11 / 2}^{\circ} \\
22194_{31 / 2}-59276_{31 / 2}^{\circ}\end{array}$ & 6 \\
\hline $\begin{array}{l}\text { 2697. } 714 \\
\text { 2698. } 265 \\
\text { 2698. } 706 \\
2699.041 \\
2699.270\end{array}$ & $\begin{array}{r}80 \\
1 \\
1 \\
8 \\
1\end{array}$ & $\begin{array}{c}160 \\
1 ? \\
3 \\
12 \\
10\end{array}$ & $\begin{array}{l}37057.42 \\
37049.86 \\
37043.80 \\
37039.21 \\
37036.06\end{array}$ & $\begin{array}{l}-0.12 \\
+0.14 \\
-0.04 \\
-0.09 \\
-0.08\end{array}$ & $\begin{array}{c}a{ }^{6} \mathrm{D}_{11 / 2}-z^{2} \mathrm{~S}_{01 / 2}^{\circ} \\
20039_{31 / 2}-57089^{\circ 1 / 2} \\
23234_{41 / 2}-60278_{41 / 2}^{\circ} \\
26227_{21 / 2}-63266_{31 / 2}^{\circ} \\
27273_{31 / 2}-64310_{21 / 2}^{\circ}\end{array}$ & res \\
\hline $\begin{array}{l}2700.320 \\
2701.036 \\
2701.485 \\
2702.115 \\
2702.188 \\
2703.066\end{array}$ & $\begin{array}{l}3 \\
8 A \\
20 \\
25 \\
4 \\
8\end{array}$ & $\begin{array}{r}50 \\
10 \\
60 \\
250 \\
? \\
60\end{array}$ & $\begin{array}{l}37021.67 \\
37011.85 \\
37005.70 \\
36997.07 \\
36996.07 \\
36984.05\end{array}$ & $\begin{array}{l}+0.09 \\
-0.29 \\
-0.11 \\
+0.01 \\
-0.05 \\
+0.08\end{array}$ & $\begin{array}{r}23234_{41 / 2}-60256_{31 / 2}^{\circ} \\
28631_{31 / 2}-656444_{11 / 2}^{\circ} \\
a^{4} \mathrm{~F}_{41 / 2}-51863_{31 / 2}^{\circ} \\
19442_{61 / 2}-56439_{61 / 2}^{\circ} \\
a^{4} \mathrm{P}_{11 / 2}-47588_{11 / 2}^{\circ} \\
23234_{41 / 2}-60218^{\circ} 1 \frac{1}{2}\end{array}$ & res \\
\hline $\begin{array}{l}2703.118 \\
2703.467 \\
2703.698 \\
2703.829 \\
2705.599\end{array}$ & $\begin{array}{r}8 \\
12 \\
1 \\
10\end{array}$ & $\begin{array}{r}10 \\
120 \\
5 \\
1 \\
35\end{array}$ & $\begin{array}{l}36983.36 \\
36978.57 \\
36975.41 \\
36973.62 \\
36949.43\end{array}$ & $\begin{array}{l}-0.04 \\
+0.13 \\
+0.05 \\
-0.02 \\
-0.11\end{array}$ & $\begin{array}{r}a^{4} \mathrm{~F}_{21 / 2}-48284^{\circ}{ }^{11 / 2} \\
31100_{51 / 2}-68078_{61 / 2}^{\circ} \\
19637_{21 / 2}-56612_{31 / 2}^{\circ} \\
23450_{21 / 2}-604244_{31 / 2}^{\circ} \\
20780_{41 / 2}-57729_{31 / 2}^{\circ}\end{array}$ & 5 \\
\hline $\begin{array}{l}2705.895 \\
2706.702 \\
2706.733 \\
2707.067 \\
2708.360\end{array}$ & $4 A$ & $\begin{array}{r}12 \\
? \\
50 \\
25 \\
2\end{array}$ & $\begin{array}{l}36945.40 \\
36934.38 \\
36933.95 \\
36929.40 \\
36911.77\end{array}$ & $\begin{array}{r}0.00 \\
-0.01 \\
+0.04 \\
+0.01 \\
-0.27\end{array}$ & $\begin{array}{r}23046_{31 / 2}-59992_{21 / 2}^{\circ} \\
a^{6} \mathrm{~S}_{21 / 2}-443544^{\circ}{ }^{\circ} / 2 \\
19442_{61 / 2}-56376_{51 / 2}^{\circ} \\
29341_{41 / 2}-66270_{41 / 2}^{\circ} \\
31100_{51 / 2}-68012_{51 / 2}^{\circ}\end{array}$ & 6 \\
\hline $\begin{array}{l}2709.582 \\
2710.678 \\
2710.792 \\
2711.336 \\
2712.704\end{array}$ & $\begin{array}{r}20 \\
2 \\
5 \\
5\end{array}$ & $\begin{array}{r}80 \\
1 \\
40 \\
3 \\
50\end{array}$ & $\begin{array}{l}36895.12 \\
36880.20 \\
36878.65 \\
36871.26 \\
36852.66\end{array}$ & $\begin{array}{l}-0.09 \\
-0.17 \\
-0.03 \\
-0.16 \\
-0.01\end{array}$ & $\begin{array}{r}a^{4} \mathrm{D}_{21 / 2}-51863_{31 / 2}^{\circ} \\
a^{4} \mathrm{~F}_{31 / 2}-50292_{21 / 2}^{\circ} \\
a^{4} \mathrm{G}_{21 / 2}-53113_{21 / 2}^{\circ} \\
28118_{21 / 2}^{\circ}-64990_{21 / 2}^{\circ} \\
23803_{31 / 2}-60656_{21 / 2}^{\circ}\end{array}$ & 6 res \\
\hline $\begin{array}{l}2713.478 \\
2714.016 \\
2714.950 \\
2715.346\end{array}$ & $\begin{array}{r}4 \\
1 \\
25\end{array}$ & $\begin{array}{r}2 \\
1 \\
5 \\
80\end{array}$ & $\begin{array}{l}36842.15 \\
36834.85 \\
36822.18 \\
36816.81\end{array}$ & $\begin{array}{l}-0.29 \\
-0.40 \\
-0.16 \\
-0.02\end{array}$ & $\begin{array}{r}a^{4} \mathrm{~F}_{11 / 2}-45553_{11 / 2}^{\circ} \\
20039_{31 / 2}-5684_{421 / 2} \\
23046_{31 / 2}-59869_{31 / 2}^{\circ} \\
a^{4} \mathrm{G}_{41 / 2}-53369_{41 / 2}^{\circ}\end{array}$ & 6 \\
\hline 2716. 020 & 2 & 3 & 36807.67 & $\begin{array}{l}-0.11 \\
-0.09\end{array}$ & $\begin{array}{l}19276_{21 / 2}-56084_{11 / 2} \\
26158_{41 / 2}-62966_{51 / 2}^{\circ}\end{array}$ & \\
\hline $\begin{array}{l}2716.147 \\
2716.322 \\
2716.890 \\
2717.180 \\
2717.702\end{array}$ & $\begin{array}{l}3 \\
30 \\
35 \mathrm{~A} \\
10 \\
3\end{array}$ & $\begin{array}{l}15 \\
80 \\
35 \\
40 \\
18\end{array}$ & $\begin{array}{l}36805.95 \\
36803.58 \\
36795.90 \\
36791.96 \\
36784.90\end{array}$ & $\begin{array}{r}0.00 \\
-0.09 \\
-0.17 \\
-0.08 \\
-0.03\end{array}$ & $\begin{array}{r}23450_{21 / 2}-60256_{31 / 2}^{\circ} \\
a^{4} \mathrm{D}_{11 / 2}-51438_{21 / 2}^{\circ} \\
20455_{11 / 2}-57252_{21 / 2}^{\circ} \\
a^{4} \mathrm{G}_{51 / 2}-54229_{51 / 2}^{\circ} \\
a^{4} \mathrm{G}_{41 / 2}-53338_{31 / 2}^{\circ}\end{array}$ & 6 res \\
\hline $\begin{array}{l}\text { 2718. } 044 \\
2718.257 \\
2719.232 \\
2719.392 \\
2719.798\end{array}$ & $\begin{array}{r}30 \\
8\end{array}$ & $\begin{array}{r}120 \\
2 \\
3 \\
10 \\
1\end{array}$ & $\begin{array}{l}36780.26 \\
36777.38 \\
36764.20 \\
36762.03 \\
36756.54\end{array}$ & $\begin{array}{r}-0.04 \\
+0.06 \\
-0.09 \\
0.00 \\
+0.06\end{array}$ & $\begin{array}{r}a^{4} \mathrm{G}_{31 / 2}-53369_{41 / 2}^{\circ} \\
28118_{21 / 2}-64896_{31 / 2}^{\circ} \\
a^{6} \mathrm{D}_{21 / 2}-39936_{21 / 2}^{\circ} \\
24804_{31 / 2}-61566_{21 / 2}^{\circ} \\
27273_{31 / 2}-64030_{21 / 2}^{\circ}\end{array}$ & 4 \\
\hline $\begin{array}{l}\text { 2720. } 404 \\
2720.594 \\
2721.850 \\
2722.805 \\
2723.704\end{array}$ & $\begin{array}{r}6 \\
4 \\
4 \\
20 \\
3\end{array}$ & $\begin{array}{r}30 \\
40 \\
15 \\
70 \\
5\end{array}$ & $\begin{array}{l}36748.36 \\
36745.80 \\
36728.83 \\
36715.95 \\
36703.84\end{array}$ & $\begin{array}{l}-0.04 \\
+0.04 \\
-0.04 \\
-0.06 \\
-0.07\end{array}$ & $\begin{array}{r}a^{4} \mathrm{G}_{31 / 2}-53338_{31 / 2}^{\circ} \\
a^{4} \mathrm{~F}_{11 / 2}-45457^{\circ}-51 / 2 \\
20039_{31 / 2}-56768_{31 / 2}^{\circ} \\
a^{4} \mathrm{D}_{31 / 2}-51863_{31 / 2}^{\circ} \\
18000_{31 / 2}-547044_{21 / 2}^{\circ}\end{array}$ & 6 \\
\hline $\begin{array}{l}2724.081 \\
2725.457 \\
2726.445 \\
2728.880 \\
2729.532\end{array}$ & $\begin{array}{l}5 \\
\\
3 \\
1 \\
1\end{array}$ & $\begin{array}{r}60 \\
8 \\
4 \\
6 \\
6\end{array}$ & $\begin{array}{l}36698.75 \\
36680.23 \\
36666.94 \\
36634.22 \\
36625.48\end{array}$ & $\begin{array}{l}-0.04 \\
+0.01 \\
+0.04 \\
-0.05 \\
+0.08\end{array}$ & $\begin{array}{r}23234_{41 / 2}-59933_{31 / 2}^{\circ} \\
19404_{01 / 2}-56084_{11 / 2}^{\circ} \\
a^{4} \mathrm{G}_{21 / 2}-52901_{31 / 2}^{\circ} \\
23234_{41 / 2}-59869_{31 / 2}^{\circ} \\
28377_{51 / 2}-65003_{41 / 2}^{\circ}\end{array}$ & \\
\hline
\end{tabular}


TABLE 3. Classified lines of W II-Continued

\begin{tabular}{|c|c|c|c|c|c|c|}
\hline \multirow{2}{*}{$\begin{array}{c}\text { Wavelength } \\
(\AA)\end{array}$} & \multicolumn{2}{|c|}{ Intensity } & \multicolumn{2}{|c|}{ Wave number $\left(\mathrm{cm}^{-1}\right)$} & \multirow{2}{*}{ Combination } & \multirow{2}{*}{$\begin{array}{l}\text { Zeeman } \\
\text { effect }\end{array}$} \\
\hline & Arc & Spark & Observed & $O-C$ & & \\
\hline Air & & & & & & \\
\hline $\begin{array}{l}2729.620 \\
2729.936 \\
2730.846 \\
2732.078 \\
2732.378\end{array}$ & $\begin{array}{r}25 \\
5 \\
3 \\
2 \\
4\end{array}$ & $\begin{array}{r}75 \\
30 \\
8 \\
8 \\
4\end{array}$ & $\begin{array}{l}36624.30 \\
36620.06 \\
36607.86 \\
36591.35 \\
36587.33\end{array}$ & $\begin{array}{l}-0.06 \\
+0.02 \\
-0.11 \\
+0.05 \\
-0.09\end{array}$ & $\begin{array}{r}a^{4} \mathrm{P}_{01 / 2}-45457_{01 / 2} \\
a^{4} \mathrm{D}_{11 / 2}-512544^{11 / 2} \\
22139_{21 / 2}-58747^{11 / 2} \\
28377_{51 / 2}-64969_{51 / 2}^{\circ} \\
a^{4} \mathrm{P}_{11 / 2}-47179_{11 / 2}^{\circ}\end{array}$ & res \\
\hline $\begin{array}{l}2733.448 \\
2733.704 \\
2734.627 \\
2734.737 \\
2735.784\end{array}$ & $\begin{array}{l}5 \\
2\end{array}$ & $\begin{array}{r}35 \\
3 \\
3 \\
40 \\
10\end{array}$ & $\begin{array}{l}36573.01 \\
36569.58 \\
36557.24 \\
36555.77 \\
36541.78\end{array}$ & $\begin{array}{r}+0.01 \\
-0.01 \\
0.00 \\
-0.10 \\
+0.08\end{array}$ & $\begin{array}{l}20039_{31 / 2}-56612_{31 / 2}^{\circ} \\
22139_{21 / 2}-58709_{31 / 2}^{\circ} \\
26158_{11 / 2}-62715_{11 / 2}^{\circ} \\
24804_{31 / 2}^{\circ}-61360_{41 / 2}^{\circ} \\
23450_{21 / 2}-59992_{21 / 2}^{\circ}\end{array}$ & 6 \\
\hline $\begin{array}{l}2737.135 \\
2737.756 \\
2737.840 \\
2738.962 \\
2739.080\end{array}$ & $\begin{array}{l}1 \\
2 \\
1 \\
4\end{array}$ & $\begin{array}{l}3 \\
5 \\
3 \\
5 \\
4\end{array}$ & $\begin{array}{l}36523.74 \\
36515.46 \\
36514.34 \\
36499.38 \\
36497.81\end{array}$ & $\begin{array}{r}-0.11 \\
-0.02 \\
0.00 \\
+0.06 \\
-0.06\end{array}$ & 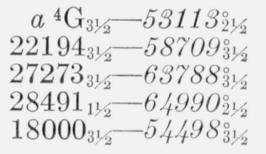 & 7 \\
\hline $\begin{array}{l}2739.140 \\
2739.384 \\
2740.187 \\
2740.801 \\
2742.471\end{array}$ & $\begin{array}{r}4 \\
6 \\
6 \\
20 \\
15\end{array}$ & $\begin{array}{r}15 \\
30 \\
20 \\
200 \\
30\end{array}$ & $\begin{array}{l}36497.01 \\
36493.76 \\
36483.06 \\
36474.90 \\
36452.68\end{array}$ & $\begin{array}{l}-0.04 \\
-0.04 \\
-0.10 \\
+0.03 \\
+0.07\end{array}$ & $\begin{array}{l}18990_{11 / 2}-55488_{11 / 2}^{\circ} \\
22194_{31 / 2}-58687_{41 / 2}^{\circ} \\
23450_{21 / 2}-59933_{31 / 2}^{\circ} \\
23803_{31 / 2}-60278_{41 / 2}^{\circ} \\
23803_{31 / 2}-60256_{31 / 2}^{\circ}\end{array}$ & $\begin{array}{l}4 \\
6 \text { res }\end{array}$ \\
\hline $\begin{array}{l}2742.903 \\
2745.036 \\
2748.312 \\
2748.418 \\
2750.023\end{array}$ & $\begin{array}{r}8 \\
2 \\
20 \\
2 \\
1\end{array}$ & $\begin{array}{r}40 \\
30 \\
15 \\
12 \\
9\end{array}$ & $\begin{array}{l}36446.94 \\
36418.62 \\
36375.21 \\
36373.82 \\
36352.61\end{array}$ & $\begin{array}{l}+0.02 \\
-0.02 \\
+0.09 \\
-0.08 \\
+0.07\end{array}$ & $\begin{array}{l}19637_{21 / 2}-56084_{11 / 2}^{\circ} \\
23450_{21 / 2}-59869_{31 / 2}^{\circ} \\
18000_{31 / 2}-54375_{21 / 2}^{\circ} \\
20039_{31 / 2}-56413_{11 / 2}^{\circ} \\
23046_{31 / 2}-59399_{41 / 2}^{\circ}\end{array}$ & $\begin{array}{l}4 \text { res } \\
\text { res }\end{array}$ \\
\hline $\begin{array}{l}2750.325 \\
2750.728 \\
2750.880 \\
2752.242 \\
2752.360\end{array}$ & $\begin{array}{c}10 \\
2 \\
3 \\
5 \\
1 ?\end{array}$ & $\begin{array}{r}20 \\
15 \\
12 \\
30 \\
2\end{array}$ & $\begin{array}{l}36348.60 \\
36343.28 \\
36341.28 \\
36323.28 \\
36321.72\end{array}$ & $\begin{array}{r}0.00 \\
+0.04 \\
-0.04 \\
-0.03 \\
+0.03\end{array}$ & $\begin{array}{c}a^{4} \mathrm{G}_{41 / 2}-52901_{31 / 2}^{\circ} \\
29341_{41 / 2}-65684_{51 / 2}^{\circ} \\
25209_{41 / 2}-61550_{31 / 2}^{\circ} \\
23955_{51 / 2}-60278_{41 / 2}^{\circ} \\
19070_{41 / 2}-55392_{41 / 2}^{\circ}\end{array}$ & res \\
\hline $\begin{array}{l}2753.094 \\
2753.320 \\
2753.826 \\
2754.695 \\
2755.036\end{array}$ & $\begin{array}{l}6 \\
1 \\
1\end{array}$ & $\begin{array}{r}6 \\
25 \\
6 \\
2 \\
3\end{array}$ & $\begin{array}{l}36312.03 \\
36309.05 \\
36302.37 \\
36290.93 \\
36286.44\end{array}$ & $\begin{array}{l}-0.04 \\
-0.03 \\
-0.07 \\
-0.08 \\
-0.14\end{array}$ & $\begin{array}{r}a^{4} \mathrm{G}_{31 / 2}-52901_{31 / 2}^{\circ} \\
20780_{41 / 2}-57089_{41 / 2}^{\circ} \\
29341_{41 / 2}-65644^{\circ} 1^{1 / 2} \\
a^{4} \mathrm{D}_{31 / 2}-51438_{21 / 2}^{\circ} \\
a^{4} \mathrm{D}_{21 / 2}-51254_{11 / 2}^{\circ}\end{array}$ & 7 \\
\hline $\begin{array}{l}2755.661 \\
2756.776 \\
2757.710 \\
2758.182 \\
2758.333\end{array}$ & 18 & $\begin{array}{r}6 \\
20 \\
6 \\
4 \\
20\end{array}$ & $\begin{array}{l}36278.21 \\
36263.54 \\
36251.26 \\
36245.05 \\
36234.07\end{array}$ & $\begin{array}{r}-0.09 \\
+0.10 \\
+0.13 \\
+0.17 \\
-0.04\end{array}$ & $\begin{array}{r}26158_{41 / 2}-62437_{41 / 2}^{\circ} \\
23955_{51 / 2}-60218^{\circ}{ }^{1 / 2} \\
24804_{31 / 2}-61055_{41 / 2}^{\circ} \\
22503_{11 / 2}-587_{4} 7^{\circ}{ }^{1 / 2} \\
a^{6} \mathrm{D}_{41 / 2}-42990_{31 / 2}^{\circ}\end{array}$ & res \\
\hline $\begin{array}{l}2759.338 \\
2760.691 \\
2760.742 \\
2761.587 \\
2761.837\end{array}$ & $\begin{array}{l}1 \\
3 \\
12 A \\
40 \\
2\end{array}$ & $\begin{array}{r}10 \\
15 \\
10 \\
100 \\
6\end{array}$ & $\begin{array}{l}36229.87 \\
36212.11 \\
36211.44 \\
36200.37 \\
36197.09\end{array}$ & $\begin{array}{r}-0.14 \\
-0.03 \\
-0.05 \\
0.00 \\
+0.08\end{array}$ & $\begin{array}{r}23046_{31 / 2}-5927_{31 / 2}^{\circ} \\
22535_{01 / 2}-584_{4} 7_{11 / 2}^{\circ} \\
19276_{21 / 2}-55488_{11 / 2}^{\circ} \\
a^{4} \mathrm{~F}_{11 / 2}-44911_{11 / 2}^{\circ} \\
22139_{21 / 2}-58336_{21 / 2}^{\circ}\end{array}$ & $\begin{array}{l}7 \\
6\end{array}$ \\
\hline $\begin{array}{l}2762.499 \\
2764.263 \\
2764.768 \\
2765.340 \\
2765.986\end{array}$ & $\begin{array}{r}6 \\
200 \\
8 \\
2\end{array}$ & $\begin{array}{r}15 \\
400 \\
50 \\
10 \\
1\end{array}$ & $\begin{array}{l}36188.42 \\
36165.33 \\
36158.72 \\
36151.24 \\
36142.80\end{array}$ & $\begin{array}{l}+0.06 \\
-0.02 \\
+0.16 \\
-0.02 \\
-0.10\end{array}$ & $\begin{array}{c}23803_{31 / 2}-59992_{21 / 2}^{\circ} \\
a^{6} \mathrm{D}_{01 / 2}-z^{6} \mathrm{~F}_{01 / 2}^{\circ} \\
26929_{51 / 2}^{\circ}-6308 \%^{\circ}{ }^{\circ 1 / 2} \\
25209_{411 / 2}-61360_{41 / 2}^{\circ} \\
22194_{31 / 2}-58336_{21 / 2}^{\circ}\end{array}$ & $\begin{array}{l}5 \\
6\end{array}$ \\
\hline $\begin{array}{l}2766.318 \\
2766.981 \\
2767.967 \\
2768.326 \\
2770.496\end{array}$ & $\begin{array}{r}1 \\
4 \\
4 \\
30 \\
2\end{array}$ & $\begin{array}{r}35 \\
35 \\
25 \\
50 \\
5\end{array}$ & $\begin{array}{l}\text { 36138. } 46 \\
36129.80 \\
36116.93 \\
36112.25 \\
36083.96\end{array}$ & $\begin{array}{r}-0.11 \\
-0.02 \\
-0.08 \\
0.00 \\
+0.03\end{array}$ & $\begin{array}{r}28377_{51 / 2}-64516_{41 / 2}^{\circ} \\
23803_{31 / 2}-59933_{31 / 2}^{\circ} \\
25209_{41 / 2}-61326_{31 / 2}^{\circ} \\
a{ }^{4} \mathrm{~F}_{21 / 2}-1 / 4413_{21 / 2}^{\circ} \\
19404_{01 / 2}^{\circ}-55488_{11 / 2}^{\circ}\end{array}$ & $\begin{array}{l}7 \\
6 \\
6\end{array}$ \\
\hline
\end{tabular}


TABLE 3. Classified lines of $\mathrm{W} \mathrm{II}$-Continued

\begin{tabular}{|c|c|c|c|c|c|c|}
\hline \multirow{2}{*}{$\begin{array}{c}\text { Wavelength } \\
\text { (A) }\end{array}$} & \multicolumn{2}{|c|}{ Intensity } & \multicolumn{2}{|c|}{ Wave number $\left(\mathrm{cm}^{-1}\right)$} & \multirow{2}{*}{ Combination } & \multirow{2}{*}{$\begin{array}{c}\text { Zeeman } \\
\text { effect }\end{array}$} \\
\hline & Arc & Spark & Observed & $O-C$ & & \\
\hline \multicolumn{7}{|l|}{ Air } \\
\hline $\begin{array}{l}2771.005 \\
2771.531 \\
2771.927 \\
2772.658 \\
2773.849\end{array}$ & $\begin{array}{l}5 \\
5 \\
2 \\
2 \\
5\end{array}$ & $\begin{array}{r}20 \\
3 \\
6 \\
8 \\
25\end{array}$ & $\begin{array}{l}36077.34 \\
36070.50 \\
36065.34 \\
36055.83 \\
36040.35\end{array}$ & $\begin{array}{l}-0.09 \\
+0.02 \\
+0.04 \\
-0.01 \\
-0.09\end{array}$ & $\begin{array}{r}a^{4} \mathrm{D}_{21 / 2}-51045_{31 / 2}^{\circ} \\
30633_{41 / 2}^{\circ}-66703_{51 / 2}^{\circ} \\
23803_{31 / 2}-59869_{31 / 2}^{\circ} \\
18000_{31 / 2}^{\circ}-54056_{41 / 2}^{\circ} \\
a^{4} \mathrm{G}_{21 / 2}-52275_{31 / 2}^{\circ}\end{array}$ & \\
\hline $\begin{array}{l}2774.092 \\
2774.433 \\
2774.988 \\
2775.878 \\
2776.509\end{array}$ & $\begin{array}{r}? \\
6 \\
1 \\
20\end{array}$ & $\begin{array}{l}30 \\
10 ? \\
20 \\
5 \\
100\end{array}$ & $\begin{array}{l}\text { 36037. } 20 \\
36032.76 \\
36025.56 \\
36014.01 \\
36005.84\end{array}$ & $\begin{array}{r}+0.04 \\
+0.01 \\
+0.02 \\
0.00 \\
+0.01\end{array}$ & $\begin{array}{r}26929_{51 / 2}-62966_{51 /}^{\circ} \\
a^{4} \mathrm{P}_{11 / 2}-46625_{01 / 2}^{\circ} \\
18000_{31 / 2}-54026_{21 / 2}^{\circ} \\
a^{4} \mathrm{G}_{41 / 2}-52567^{\circ 11 / 2} \\
a^{4} \mathrm{~F}_{41 / 2}-50863_{41 / 2}^{\circ}\end{array}$ & 6 \\
\hline $\begin{array}{l}\text { 2777. } 536 \\
2777.870 \\
2778.124 \\
2778.281 \\
2778.429\end{array}$ & $\begin{array}{l}2 \\
8 \\
5\end{array}$ & $\begin{array}{r}10 \\
30 \\
2 \\
3 \\
6\end{array}$ & $\begin{array}{l}35992.51 \\
35988.18 \\
35984.90 \\
35982.86 \\
35980.95\end{array}$ & $\begin{array}{l}+0.07 \\
-0.05 \\
+0.06 \\
-0.01 \\
-0.17\end{array}$ & $\begin{array}{r}27273_{31 / 2}-63266_{31 / 2}^{\circ} \\
20780_{41 / 2}-56768_{31 / 2}^{\circ} \\
29341_{41 / 2}-65326_{51 / 2}^{\circ} \\
24918_{11 / 2}-60900_{21 / 2}^{\circ} \\
a^{4} \mathrm{D}_{01 / 2}-491540_{11 / 2}^{\circ}\end{array}$ & \\
\hline $\begin{array}{l}2778.694 \\
2780.285 \\
2782.142 \\
2783.979 \\
2784.290\end{array}$ & $\begin{array}{l}20 \\
60 A \\
20 \\
2 \\
2\end{array}$ & $\begin{array}{r}80 \\
40 \\
80 \\
6 \\
15\end{array}$ & $\begin{array}{l}35977.51 \\
35956.94 \\
35932.93 \\
35909.22 \\
35905.21\end{array}$ & $\begin{array}{l}+0.03 \\
+0.05 \\
-0.02 \\
-0.19 \\
-0.04\end{array}$ & $\begin{array}{c}a^{4} \mathrm{G}_{31 / 2}-52567^{\circ}{ }^{1 / 12} \\
a^{6} \mathrm{D}_{21 / 2}-z^{6} \mathrm{~F}_{11 / 2}^{\circ} \\
a^{4} \mathrm{G}_{51 / 2}-53969_{41 / 2}^{\circ} \\
24991_{11 / 2}-60900_{21 / 2}^{\circ} \\
20534_{51 / 2}^{\circ}-56439_{61 / 2}^{\circ}\end{array}$ & $\begin{array}{l}\text { res } \\
\text { res }\end{array}$ \\
\hline $\begin{array}{l}2785.126 \\
2785.634 \\
2786.300 \\
2786.340 \\
2788.403\end{array}$ & $\left.\begin{array}{r}1 \\
12 \\
4 \\
3\end{array}\right\}$ & $\begin{array}{r}3 \\
50 \\
20 \\
2\end{array}$ & $\begin{array}{l}35894.43 \\
35887.90 \\
35879.31 \\
35878.80 \\
35852.25\end{array}$ & $\begin{array}{r}-0.11 \\
0.00 \\
+0.02 \\
-0.06 \\
+0.07\end{array}$ & $\begin{array}{r}25672_{21 / 2}-61566_{21 / 2}^{\circ} \\
19070_{41 / 2}-54958_{51 / 2}^{\circ} \\
20534_{51 / 2}-56413_{41 / 2}^{\circ} \\
a^{4} \mathrm{~F}_{21 / 2}-47179_{11 / 2}^{\circ} \\
a^{4} \mathrm{G}_{21 / 2}-52087_{21 / 2}^{\circ}\end{array}$ & 7 \\
\hline $\begin{array}{l}2788.536 \\
2789.197 \\
2790.160 \\
2790.433 \\
2790.998\end{array}$ & $\begin{array}{r}4 \\
10 \\
2 \\
5 \\
3\end{array}$ & $\begin{array}{r}6 \\
20 \\
18 \\
50 \\
3\end{array}$ & $\begin{array}{l}35850.54 \\
35842.04 \\
35829.67 \\
35826.17 \\
35818.92\end{array}$ & $\begin{array}{l}-0.09 \\
-0.06 \\
-0.03 \\
-0.14 \\
-0.08\end{array}$ & $\begin{array}{l}19637_{21 / 2}-55488_{11 / 2}^{\circ} \\
20534_{51 / 2}-56376_{51 / 2}^{\circ} \\
28377_{51 / 2}-64207_{41 / 2}^{\circ} \\
23450_{21 / 2}-5927_{31 / 2}^{\circ} \\
28491_{11 / 2}-64310_{21 / 2}^{\circ}\end{array}$ & $\begin{array}{l}7 \\
\text { res }\end{array}$ \\
\hline $\begin{array}{l}\text { 2791. } 740 \\
2791.850 \\
2793.622 \\
2796.674 \\
2796.866\end{array}$ & $\begin{array}{l}2 \\
\frac{1}{1} \\
3\end{array}$ & $\begin{array}{l}5 \\
1 \\
4 \\
3 \\
6\end{array}$ & $\begin{array}{l}35809.40 \\
35807.98 \\
35785.28 \\
35746.23 \\
35743.77\end{array}$ & $\begin{array}{l}-0.08 \\
-0.02 \\
+0.06 \\
-0.11 \\
-0.15\end{array}$ & $\begin{array}{r}a^{4} \mathrm{D}_{01 / 2}-48982^{\circ}{ }^{11 / 2} \\
a^{4} \mathrm{P}_{21 / 2}-49242^{\circ 11 / 2} \\
26929_{51 / 2}-627146^{1 / 2} \\
19276_{21 / 2}-55022_{31 / 2}^{\circ} \\
a^{4} \mathrm{~F}_{11 / 2}-44455_{01 / 2}^{\circ 1}\end{array}$ & res \\
\hline $\begin{array}{l}\text { 2797. } 298 \\
2797.879 \\
2798.353 \\
2799.042 \\
2799.248\end{array}$ & $\begin{array}{r}20 \\
1\end{array}$ & $\begin{array}{r}2 \\
1 \\
3 \\
100\end{array}$ & $\begin{array}{l}35738.25 \\
35730.83 \\
35724.78 \\
35715.98 \\
35713.36\end{array}$ & $\begin{array}{l}-0.16 \\
-0.27 \\
-0.06 \\
-0.05 \\
-0.29\end{array}$ & $\begin{array}{r}24918_{11 / 2}-60656_{21 / 2}^{\circ} \\
25169_{11 / 2}-60900_{21 / 2}^{\circ} \\
28631_{31 / 2}-64356_{31 / 2}^{\circ} \\
a^{4} \mathrm{D}_{31 / 2}-50863_{41 / 2}^{\circ} \\
18990_{11 / 2}^{\circ}-54704^{\circ}{ }^{11 / 2}\end{array}$ & 5 \\
\hline $\begin{array}{l}\text { 2799. } 312 \\
\text { 2801. } 058 \\
\text { 2801. } 430 \\
\text { 3802. } 705 \\
\text { 2803. } 068\end{array}$ & $\begin{array}{l}1 \\
4\end{array}$ & $\begin{array}{r}30 \\
10 \\
3 \\
4\end{array}$ & $\begin{array}{l}35712.54 \\
35690.28 \\
35685.54 \\
35669.31 \\
35664.70\end{array}$ & $\begin{array}{l}-0.02 \\
-0.14 \\
-0.07 \\
+0.01 \\
-0.25\end{array}$ & $\begin{array}{r}a^{4} \mathrm{~F}_{31 / 2}-49124^{\circ}{ }^{\circ} 1 / 2 \\
a^{4} \mathrm{P}_{21 / 2}-49122^{\circ}{ }^{31 / 2} \\
a^{4} \mathrm{G}_{31 / 2}-52275^{\circ}{ }^{11 / 2} \\
28118_{21 / 2}-63788^{\circ}{ }^{1 / 2} \\
24991_{11 / 2}-60656^{\circ}{ }^{11 / 2}\end{array}$ & 6_res \\
\hline $\begin{array}{l}\text { 2803. } 233 \\
\text { 2803. } 302 \\
\text { 2803. } 604 \\
\text { 2803. } 682 \\
\text { 2803. } 909\end{array}$ & $\begin{array}{l}4 \\
1 \\
8\end{array}$ & $\begin{array}{r}10 \\
3 \\
25 \\
3 \\
5\end{array}$ & $\begin{array}{l}\text { 35662. } 60 \\
35661.71 \\
35657.87 \\
35656.88 \\
35654.00\end{array}$ & $\begin{array}{l}-0.02 \\
+0.07 \\
-0.10 \\
+0.01 \\
-0.13\end{array}$ & $\begin{array}{r}22194_{31 / 2}-57856_{21 / 2}^{\circ} \\
29341_{41 / 2}-65003_{41 / 2}^{\circ} \\
a^{4} \mathrm{D}_{11 / 2}-50292_{21 / 2}^{\circ} \\
23234_{41 / 2}-58891_{51 / 2}^{\circ} \\
25672_{21 / 2}-61326_{31 / 2}^{\circ}\end{array}$ & res \\
\hline $\begin{array}{l}\text { 2804. } 922 \\
2805.177 \\
2805.546 \\
\text { 2805. } 936 \\
2806.392\end{array}$ & $\begin{array}{r}20 \\
3\end{array}$ & $\begin{array}{r}12 \\
15 \\
7 \\
120 \\
4\end{array}$ & $\begin{array}{l}\text { 35641. } 12 \\
35637.88 \\
35633.20 \\
35628.24 \\
35622.45\end{array}$ & $\left\{\begin{array}{l}+0.04 \\
-0.05 \\
-0.06 \\
-0.13 \\
+0.05 \\
-0.07\end{array}\right.$ & $\begin{array}{r}23046_{31 / 2}-58687^{\circ}{ }^{1 / 1} 2 \\
30633_{41 / 2}-66270_{41 / 2}^{\circ} \\
20780_{41 / 2}-56413_{41 / 2}^{\circ} \\
20455_{11 / 2}-56084^{\circ}{ }^{11 / 2} \\
a^{4} \mathrm{G}_{21 / 2}-51863_{31 / 2}^{\circ} \\
a^{4} \mathrm{P}_{01 / 2}-44455_{01 / 2}^{\circ}\end{array}$ & $\begin{array}{l}6 \\
6\end{array}$ \\
\hline
\end{tabular}


TABLE 3. Classified lines of $\mathrm{W}$ II-Continued

\begin{tabular}{|c|c|c|c|c|c|c|}
\hline \multirow{2}{*}{$\begin{array}{l}\text { Wavelength } \\
\text { (囚) }\end{array}$} & \multicolumn{2}{|c|}{ Intensity } & \multicolumn{2}{|c|}{ Wave number $\left(\mathrm{cm}^{-1}\right)$} & \multirow{2}{*}{ Combination } & \multirow{2}{*}{$\begin{array}{c}\text { Zeeman } \\
\text { effect }\end{array}$} \\
\hline & Arc & Spark & Observed & $O-C$ & & \\
\hline \multicolumn{7}{|l|}{ Air } \\
\hline $\begin{array}{l}\text { 2806. } 626 \\
2808.474 \\
2808.956 \\
2810.085 \\
2811.743\end{array}$ & $\begin{array}{l}3 \\
4 \\
3 \\
1\end{array}$ & $\begin{array}{r}10 \\
40 \\
50 \\
3 \\
6\end{array}$ & $\begin{array}{l}35619.48 \\
35596.04 \\
35589.93 \\
35575.64 \\
35554.65\end{array}$ & $\begin{array}{r}+0.01 \\
-0.03 \\
-0.02 \\
0.00 \\
-0.01\end{array}$ & $\begin{array}{l}24804_{31 / 2}-604244_{31 / 2}^{\circ} \\
20780_{41 / 2}-56376_{51 / 2}^{\circ} \\
22139_{21 / 2}-57729^{\circ}{ }^{\circ 1 / 2} \\
28631_{31 / 2}-64207_{41 / 2}^{\circ} \\
29341_{41 / 2}-64896_{31 / 2}^{\circ}\end{array}$ & $\begin{array}{l}6 \\
\text { res } \\
\text { res }\end{array}$ \\
\hline $\begin{array}{l}\text { 2812. } 210 \\
2813.228 \\
2814.106 \\
2814.798 \\
2815.720\end{array}$ & $\begin{array}{l}8 \\
3 \\
4\end{array}$ & $\begin{array}{r}50 \\
15 \\
2 \\
30 \\
2\end{array}$ & $\begin{array}{l}35548.76 \\
35535.90 \\
35524.81 \\
35516.07 \\
35504.44\end{array}$ & $\begin{array}{r}0.00 \\
+0.06 \\
-0.11 \\
+0.03 \\
-0.10\end{array}$ & $\begin{array}{r}a^{4} \mathrm{P}_{21 / 2}-48982_{11 / 2}^{\circ} \\
22194_{31 / 2}-57729^{\circ}{ }^{\circ} 1 / 2 \\
19637_{21 / 2}-55162_{21 / 2}^{\circ} \\
19442_{61 / 2}-54958^{\circ}{ }^{\circ} 1 / 2 \\
22503_{11 / 2}-5807_{11 / 2}^{\circ}\end{array}$ & res \\
\hline $\begin{array}{l}\text { 2816. } 283 \\
2816.478 \\
2817.422 \\
2819.002 \\
2819.897\end{array}$ & $\begin{array}{l}6 \\
1 \\
4 \\
4\end{array}$ & $\begin{array}{r}10 \\
2 \\
3 \\
12 \\
20\end{array}$ & $\begin{array}{l}35497.35 \\
35494.90 \\
35483.00 \\
35463.11 \\
35451.85\end{array}$ & $\begin{array}{r}0.00 \\
+0.26 \\
-0.11 \\
-0.02 \\
+0.07\end{array}$ & $\begin{array}{r}a^{4} \mathrm{G}_{31 / 2}-52087^{\circ 11 / 2} \\
18990_{11 / 2}-54485_{01 / 2}^{\circ} \\
24991_{11 / 2}-6044^{4}{ }^{11 / 2} \\
a^{4} \mathrm{D}_{21 / 2}-50430_{11 / 2}^{\circ} \\
24804_{31 / 2}-60256_{31 / 2}^{\circ}\end{array}$ & $\begin{array}{l}7 \\
\text { res }\end{array}$ \\
\hline $\begin{array}{l}2820.532 \\
2821.800 \\
2822.542 \\
2824.144 \\
2824.306\end{array}$ & $\begin{array}{r}3 \\
5 \\
25 \\
4\end{array}$ & $\begin{array}{r}8 \\
35 \\
125 \\
4 \\
4\end{array}$ & $\begin{array}{l}35443.87 \\
35427.95 \\
35418.64 \\
35398.54 \\
35396.52\end{array}$ & $\begin{array}{l}-0.07 \\
-0.14 \\
-0.10 \\
+0.06 \\
-0.08\end{array}$ & $\begin{array}{r}23955_{51 / 2}-59399_{41 / 2}^{\circ} \\
19276_{21 / 2}-5470_{421 / 2}^{\circ} \\
a^{4} \mathrm{~F}_{31 / 2}-48830^{\circ} 112 \\
28631_{31 / 2}-64030_{21 / 2}^{\circ} \\
a^{4} \mathrm{P}_{21 / 2}-48830^{\circ} 1 \frac{1}{2}\end{array}$ & $\begin{array}{l}\text { res } \\
\text { res }\end{array}$ \\
\hline $\begin{array}{l}\text { 2824. } 680 \\
2824.896 \\
2825.188 \\
2826.482 \\
2827.542\end{array}$ & $\begin{array}{l}2 \\
1 \\
1 \\
3\end{array}$ & $\begin{array}{r}6 \\
7 \\
6 \\
15 \\
8\end{array}$ & $\begin{array}{l}35391.83 \\
35389.12 \\
35385.47 \\
35369.27 \\
35356.00\end{array}$ & $\begin{array}{r}-0.03 \\
+0.02 \\
-0.01 \\
0.00 \\
+0.02\end{array}$ & $\begin{array}{l}26158_{41 / 2}-61550_{31 / 2}^{\circ} \\
28491_{11 / 2}-63880_{21 / 2}^{\circ} \\
19637_{21 / 2}-55022_{31 / 2}^{\circ} \\
18000_{31 / 2}-53869_{41 / 2}^{\circ} \\
19404_{01 / 2}^{\circ}-54760_{01 / 2}^{\circ}\end{array}$ & 7 \\
\hline $\begin{array}{l}\text { 2830. } 064 \\
2831.236 \\
2831.643 \\
2832.215 \\
2832.848\end{array}$ & $\begin{array}{r}12 \\
7 \\
1\end{array}$ & $\begin{array}{r}80 \\
35 \\
2 \\
3 \\
1\end{array}$ & $\begin{array}{l}35324.50 \\
35309.88 \\
35304.80 \\
35297.67 \\
35289.80\end{array}$ & $\begin{array}{r}-0.01 \\
-0.01 \\
0.00 \\
+0.23 \\
-0.38\end{array}$ & $\begin{array}{r}a^{4} \mathrm{D}_{21 / 2}-50292_{21 / 2}^{\circ} \\
a^{4} \mathrm{G}_{41 / 2}-51863_{31 / 2}^{\circ} \\
25169_{11 / 2}^{\circ}-6047_{421 / 2} \\
23450_{21 / 2}-58747_{11 / 2}^{\circ} \\
23046_{31 / 2}-58336_{21 / 2}^{\circ}\end{array}$ & 6 \\
\hline $\begin{array}{l}\text { 2834. } 208 \\
2835.334 \\
2838.422 \\
2839.820 \\
2839.936\end{array}$ & $\begin{array}{l}8 A \\
5 \\
3 \\
1\end{array}$ & $\begin{array}{r}50 \\
5 \\
4 \\
10 \\
10\end{array}$ & $\begin{array}{l}35272.85 \\
35258.85 \\
35220.50 \\
35203.15 \\
35201.71\end{array}$ & $\begin{array}{l}-0.51 \\
-0.21 \\
-0.01 \\
-0.04 \\
-0.09\end{array}$ & $\begin{array}{r}a^{4} \mathrm{G}_{31 / 2}-51863^{\circ}{ }^{11 / 2} \\
23450_{21 / 2}-58709^{\circ}{ }^{\circ}-2 \\
a^{6} \mathrm{D}_{31 / 2}-39936_{21 / 2}^{\circ}{ }^{\circ}-51438_{21 / 2}^{\circ} \\
a^{4} \mathrm{G}_{21 / 2}-51360_{41 / 2}^{\circ} \\
26158_{41 / 2}^{\circ}-613\end{array}$ & 6 \\
\hline $\begin{array}{l}\text { 2841. } 080 \\
\text { 2842. } 460 \\
2842.700 \\
2843.045 \\
2843.449\end{array}$ & $\begin{array}{r}2 \\
2 \\
2 \\
2\end{array}$ & $\begin{array}{r}6 \\
6 \\
15 \\
2 \\
5\end{array}$ & $\begin{array}{l}35187.54 \\
35170.46 \\
35167.50 \\
35163.22 \\
35158.23\end{array}$ & $\begin{array}{l}+0.01 \\
-0.03 \\
-0.05 \\
+0.04 \\
-0.15\end{array}$ & $\begin{array}{l}24804_{31 / 2}-59992_{21 / 2}^{\circ} \\
31100_{51 / 2}-66270_{41 / 2}^{\circ} \\
26158_{41 / 2}-61326_{31 / 2}^{\circ} \\
27273_{31 / 2}-62437_{41 / 2}^{\circ} \\
19070_{41 / 2}-54229_{51 / 2}^{\circ}\end{array}$ & \\
\hline $\begin{array}{l}\text { 2843. } 603 \\
2844.426 \\
2844.500 \\
2845.725 \\
2846.357\end{array}$ & $\begin{array}{l}1 \\
2 \\
3\end{array}$ & $\begin{array}{r}12 \\
1 \\
8 \\
10 \\
1\end{array}$ & $\begin{array}{l}35156.32 \\
35146.15 \\
35145.24 \\
35130.11 \\
35122.31\end{array}$ & $\begin{array}{l}-0.02 \\
-0.09 \\
-0.07 \\
-0.02 \\
-0.25\end{array}$ & $\begin{array}{r}28631_{31 / 2}-63788_{31 / 2}^{\circ} \\
18990_{11 / 2}-54137_{11 / 2}^{\circ} \\
a{ }^{4} \mathrm{D}_{31 / 2}-50292_{21 / 2}^{\circ} \\
a^{4} \mathrm{G}_{51 / 2}-52567^{\circ}{ }_{11 / 2} \\
20039_{31 / 2}-55162_{21 / 2}^{\circ}\end{array}$ & $\begin{array}{c}6 \\
\text { res } \\
\text { res }\end{array}$ \\
\hline $\begin{array}{l}\text { 2847. } 140 \\
2848.234 \\
2849.678 \\
2850.808 \\
2851.068\end{array}$ & $35 \mathrm{~A}$ & $\begin{array}{r}18 \\
5 \\
1 \\
8 \\
4\end{array}$ & $\begin{array}{l}35112.65 \\
35099.16 \\
35081.38 \\
35067.48 \\
35064.28\end{array}$ & $\left\{\begin{array}{l}-0.17 \\
-0.14 \\
-0.13 \\
-0.04 \\
+0.25 \\
-0.19\end{array}\right.$ & $\begin{array}{l}18000_{31 / 2}-53113_{21 / 2}^{\circ} \\
19276_{21 / 2}-54375_{21 / 2}^{\circ} \\
26227_{21 / 2}-61326_{31 / 2}^{\circ} \\
19404_{01 / 2}-54485_{01 / 2}^{\circ} \\
19637_{21 / 2}-5470421 / 2 \\
24804_{31 / 2}-59869_{31 / 2}^{\circ}\end{array}$ & res \\
\hline $\begin{array}{l}2851.554 \\
2852.083 \\
2852.460 \\
2853.440 \\
2853.694\end{array}$ & $\begin{array}{l}3 \\
3\end{array}$ & $\begin{array}{r}3 \\
70 \\
1 \\
20 \\
4\end{array}$ & $\begin{array}{l}35058.30 \\
35051.80 \\
35047.17 \\
35035.13 \\
35032.02\end{array}$ & $\begin{array}{r}+0.38 \\
+0.02 \\
0.00 \\
-0.15 \\
-0.06\end{array}$ & $\begin{array}{l}22194_{31 / 2}-57252_{21 / 2}^{\circ} \\
30633_{41 / 2}-65684^{\circ} 5^{1 / 2} \\
25209_{41 / 2}-60256_{31 / 2}^{\circ} \\
18990_{11 / 2}-54026_{21 / 2}^{\circ} \\
20455_{11 / 2}^{\circ}-55488_{11 / 2}^{\circ}\end{array}$ & $\begin{array}{l}5 \\
\text { res }\end{array}$ \\
\hline
\end{tabular}


TABle 3. Classified lines of W II-Continued

\begin{tabular}{|c|c|c|c|c|c|c|}
\hline \multirow{2}{*}{$\begin{array}{l}\text { Wavelength } \\
(\AA)\end{array}$} & \multicolumn{2}{|c|}{ Intensity } & \multicolumn{2}{|c|}{ Wave number $\left(\mathrm{cm}^{-1}\right)$} & \multirow{2}{*}{ Combination } & \multirow{2}{*}{$\begin{array}{l}\text { Zeeman } \\
\text { effect }\end{array}$} \\
\hline & Arc & Spark & Observed & $O-C$ & & \\
\hline \multicolumn{7}{|l|}{ Air } \\
\hline $\begin{array}{l}2854.719 \\
2855.524 \\
2856.256 \\
2857.470 \\
2857.695\end{array}$ & 3 & $\begin{array}{r}4 \\
25 \\
2 \\
1 \\
6\end{array}$ & $\begin{array}{l}35019.44 \\
35009.56 \\
35000.60 \\
34985.72 \\
34982.97\end{array}$ & $\begin{array}{r}-0.12 \\
0.00 \\
-0.04 \\
-0.14 \\
-0.15\end{array}$ & $\begin{array}{r}a^{4} \mathrm{G}_{21 / 2}-51254_{11 / 2}^{\circ} \\
25209_{41 / 2}-60218_{51 / 2}^{\circ} \\
24991_{11 / 2}-59992_{21 / 2}^{\circ} \\
19070_{41 / 2}-54056_{41 / 2}^{\circ} \\
20039_{31 / 2}-55022_{31 / 2}^{\circ}\end{array}$ & 6 \\
\hline $\begin{array}{l}2859.484 \\
2860.898 \\
2861.058 \\
2861.21 \\
2861.518\end{array}$ & $\begin{array}{l}8 \\
\\
2 \\
2\end{array}$ & $\begin{array}{r}30 \\
12 \\
6 \\
10 \\
2\end{array}$ & $\begin{array}{l}34961.08 \\
34943.80 \\
34941.85 \\
34939.99 \\
34936.23\end{array}$ & $\begin{array}{l}-0.10 \\
-0.01 \\
-0.13 \\
-0.11\end{array}$ & $\begin{array}{c}a^{4} \mathrm{P}_{11 / 2}-45553_{11 / 2}^{\circ} \\
a^{4} \mathrm{G}_{41 / 2}-z^{6} \mathrm{~F}_{51 / 2}^{\circ} \\
23046_{31 / 2}-57986_{41 / 2}^{\circ} \\
23955_{51 / 2}^{\circ}-58891_{51 / 2}^{\circ}\end{array}$ & res \\
\hline $\begin{array}{l}\text { 2864. } 036 \\
2864.395 \\
2864.473 \\
2864.744 \\
2864.858\end{array}$ & $\begin{array}{l}1 \\
2 \\
1 \\
3\end{array}$ & $\begin{array}{r}2 \\
15 \\
8 \\
10\end{array}$ & $\begin{array}{l}34905.52 \\
34901.14 \\
34900.20 \\
34896.90 \\
34895.51\end{array}$ & $\begin{array}{l}-0.20 \\
+0.10 \\
-0.10 \\
-0.16 \\
+0.13\end{array}$ & $\begin{array}{l}23803_{31 / 2}-58709_{31 / 2}^{\circ} \\
18000_{31 / 2}-52901_{31 / 2}^{\circ} \\
28187_{61 / 2}-63087_{61 / 2}^{\circ} \\
26158_{41 / 2}-61055_{41 / 2}^{\circ} \\
22194_{31 / 2}-57089_{41 / 2}^{\circ}\end{array}$ & $\begin{array}{l}6 \\
7\end{array}$ \\
\hline $\begin{array}{l}\text { 2865. } 603 \\
2865.804 \\
2866.322 \\
\text { 2866. } 600 \\
\text { 2866. } 751\end{array}$ & $\begin{array}{l}3 \\
8 \\
4 \\
6\end{array}$ & $\begin{array}{r}6 \\
20 \\
10 \\
8 \\
20\end{array}$ & $\begin{array}{l}34886.43 \\
34884.00 \\
34877.68 \\
34874.30 \\
34872.46\end{array}$ & $\begin{array}{l}-0.05 \\
-0.04 \\
-0.09 \\
-0.04 \\
-0.06\end{array}$ & $\begin{array}{r}23450_{21 / 2}-58396_{21 / 2}^{\circ} \\
23803_{31 / 2}-58687^{\circ}{ }^{11 / 2} \\
a^{6} \mathrm{~S}_{21 / 2}-42298_{11 / 2}^{\circ} \\
a^{4} \mathrm{~F}_{21 / 2}-46175_{31 / 2}^{\circ} \\
a^{4} \mathrm{~F}_{31 / 2}-48284^{\circ}{ }^{1} 1 / 2\end{array}$ & res \\
\hline $\begin{array}{l}2866.910 \\
2867.409\end{array}$ & 6 & $\begin{array}{r}1 \\
15\end{array}$ & $\begin{array}{l}34870.53 \\
34864.46\end{array}$ & $\begin{array}{l}-0.17 \\
-0.04\end{array}$ & $\begin{array}{r}28118_{21 / 2}-62989_{21 / 2}^{\circ} \\
a^{4} \mathrm{P}_{11 / 2}-45457_{01 / 2}\end{array}$ & res \\
\hline 2867.697 & 4 & 3 & 34860. 96 & $\begin{array}{l}-0.23 \\
+0.28\end{array}$ & $\begin{array}{l}19637_{21 / 2}-54498^{11 / 2} \\
19276^{11}-54137^{\circ}\end{array}$ & \\
\hline $\begin{array}{l}2867.934 \\
2868.736\end{array}$ & $\begin{array}{l}4 \\
8\end{array}$ & $\begin{array}{l}20 \\
80\end{array}$ & $\begin{array}{l}34858.08 \\
34848.33\end{array}$ & $\begin{array}{l}+0.06 \\
-0.03\end{array}$ & $\begin{array}{r}20534_{51 / 2}-55392_{41 / 2}^{\circ} \\
a^{4} \mathrm{G}_{31 / 2}-51.438_{21 / 2}^{\circ}\end{array}$ & \\
\hline $\begin{array}{l}2870.906 \\
2871.902 \\
2873.352 \\
2873.836 \\
2874.478\end{array}$ & $\begin{array}{c}18 A \\
2 \\
2 ? \\
2\end{array}$ & $\begin{array}{l}10 \\
12 \\
10 \\
12 \\
12\end{array}$ & $\begin{array}{l}34822.00 \\
34809.92 \\
34792.35 \\
34786.50 \\
34778.72\end{array}$ & $\begin{array}{l}-0.33 \\
+0.02 \\
+0.05 \\
-0.02 \\
-0.18\end{array}$ & $\begin{array}{l}25169_{11 / 2}-59992_{21 / 2}^{\circ} \\
23046_{31 / 2}-57856_{21 / 2}^{\circ} \\
22139_{21 / 2}-56932_{11 / 2}^{\circ} \\
19442_{61 / 2}^{\circ}-54299_{51 / 2}^{\circ} \\
28187_{61 / 2}^{\circ}-62966_{51 / 2}^{\circ}\end{array}$ & res \\
\hline $\begin{array}{l}2875.110 \\
2876.684 \\
2876.934 \\
2877.827 \\
2878.083\end{array}$ & $\begin{array}{r}1- \\
20 A \\
10 A\end{array}$ & $\begin{array}{r}4 \\
2 \\
10 \\
2 \\
8\end{array}$ & $\begin{array}{l}34771.08 \\
34752.06 \\
34749.01 \\
34738.26 \\
34735.16\end{array}$ & $\begin{array}{l}-0.02 \\
+0.08 \\
+0.07 \\
-0.18 \\
+0.14\end{array}$ & $\begin{array}{l}25045_{01 / 2}-59816_{11 / 2}^{\circ} \\
25672_{21 / 2}-604243^{\circ} 1 / 2 \\
22503_{11 / 2}-57252_{21 / 2}^{\circ} \\
19637_{21 / 2}-54375_{21 / 2}^{\circ} \\
22139_{21 / 2}-56874_{21 / 2}^{\circ}\end{array}$ & $\begin{array}{l}6 \\
6\end{array}$ \\
\hline $\begin{array}{l}2878.316 \\
2880.164 \\
2881.538 \\
2882.400 \\
2883.152\end{array}$ & $\begin{array}{l}2 \\
1- \\
3 \\
3\end{array}$ & $\begin{array}{r}40 \\
8 \\
8 \\
20 \\
3\end{array}$ & $\begin{array}{l}34732.35 \\
34710.07 \\
34693.52 \\
34683.14 \\
34674.10\end{array}$ & $\begin{array}{l}-0.13 \\
-0.03 \\
+0.14 \\
+0.02 \\
+0.13\end{array}$ & $\begin{array}{l}23955_{51 / 2}-58687_{41 / 2}^{\circ} \\
28377_{51 / 2}-63087_{61 / 2}^{\circ} \\
30633_{41 / 2}-65326_{51 / 2}^{\circ} \\
23046_{31 / 2}-57729_{31 / 2}^{\circ} \\
26227_{21 / 2}-60900_{21 / 2}^{\circ}\end{array}$ & 6 \\
\hline $\begin{array}{l}\text { 2883. } 247 \\
\text { 2883. } 915 \\
2884.310 \\
2885.463 \\
2886.464\end{array}$ & $\begin{array}{l}6 \\
2 \\
1\end{array}$ & $\begin{array}{r}4 \\
2 \\
20 \\
2 \\
6\end{array}$ & $\begin{array}{l}34672.95 \\
34664.92 \\
34660.18 \\
34646.33 \\
34634.31\end{array}$ & $\begin{array}{l}+0.11 \\
+0.05 \\
+0.06 \\
-0.24 \\
-0.13\end{array}$ & $\begin{array}{c}26929_{51 / 2}-61602^{\circ}{ }^{\circ} / 2 \\
20039_{31 / 2}-54704^{\circ}{ }^{1 / 2} \\
26929_{51 / 2}-61589^{\circ}{ }^{1 / 2} \\
a^{6} \mathrm{D}_{11 / 2}-z^{6} \mathrm{~F}_{01 / 2}^{\circ} \\
28631_{31 / 2}-63266^{\circ}{ }_{31 / 2}\end{array}$ & 6 \\
\hline $\begin{array}{l}\text { 2886. } 896 \\
2886.923 \\
2888.318 \\
2888.699 \\
2889.780\end{array}$ & $\begin{array}{c}5 \\
15 A \\
4\end{array}$ & $\begin{array}{r}35\} \\
8 \\
4 \\
50\end{array}$ & $\begin{array}{l}34629.13 \\
34628.81 \\
34612.08 \\
34607.52 \\
34594.58\end{array}$ & $\begin{array}{l}+0.11 \\
+0.17 \\
+0.09 \\
-0.22 \\
-0.09\end{array}$ & $\begin{array}{c}a^{6} \mathrm{~S}_{21 / 2}-z{ }^{6} \mathrm{~F}_{21 / 2}^{\circ} \\
22139_{21 / 2}-56768_{31 / 2}^{\circ} \\
20780_{41 / 2}-55392_{41 / 2}^{\circ} \\
a^{4} D_{11 / 2}-492_{4} 2_{21 / 2}^{\circ} \\
24804_{31 / 2}-59399_{41 / 2}^{\circ}\end{array}$ & $d$ \\
\hline $\begin{array}{l}\text { 2890. } 634 \\
\text { 2891. } 456 \\
\text { 2892. } 912 \\
\text { 2894. } 924 \\
\text { 2895. } 441\end{array}$ & $\begin{array}{l}2 \\
3 \\
2 \\
2 \\
2\end{array}$ & $\begin{array}{l}25 \\
20 \\
10 \\
10 \\
10\end{array}$ & $\begin{array}{l}34584.35 \\
34574.52 \\
34557.12 \\
34533.10 \\
34526.94\end{array}$ & $\begin{array}{l}+0.01 \\
-0.01 \\
+0.02 \\
-0.04 \\
-0.02\end{array}$ & $\begin{array}{l}31100_{51 / 2}-65684_{51 / 2}^{\circ} \\
22194_{31 / 2}^{\circ}-56768_{31 / 2}^{\circ} \\
23450_{21 / 2}-5807_{11 / 2}^{\circ} \\
23803_{31 / 2}^{\circ}-58336_{21 / 2}^{\circ} \\
28187_{61 / 2}-6274_{41 / 2}^{\circ}\end{array}$ & $\begin{array}{l}6 \\
6 \\
\text { res } \\
6\end{array}$ \\
\hline
\end{tabular}


TABle 3. Classified lines of W $\mathrm{II}$-Continued

\begin{tabular}{|c|c|c|c|c|c|c|}
\hline \multirow{2}{*}{$\begin{array}{c}\text { Wavelength } \\
(\AA)\end{array}$} & \multicolumn{2}{|c|}{ Intensity } & \multicolumn{2}{|c|}{ Wave number $\left(\mathrm{cm}^{-1}\right)$} & \multirow{2}{*}{ Combination } & \multirow{2}{*}{$\begin{array}{c}\text { Zeeman } \\
\text { effect }\end{array}$} \\
\hline & Arc & Spark & Observed & $O-C$ & & \\
\hline Air & & & & & & \\
\hline $\begin{array}{l}\text { 2897. } 726 \\
2898.102 \\
\text { 2898. } 360 \\
\text { 2901. } 174 \\
\text { 2901. } 448\end{array}$ & 2 & $\begin{array}{r}3 \\
2 \\
1 \\
25 \\
3\end{array}$ & $\begin{array}{l}34499.71 \\
34495.24 \\
34492.17 \\
34458.71 \\
34455.46\end{array}$ & $\begin{array}{l}-0.11 \\
+0.19 \\
+0.06 \\
-0.12 \\
-0.12\end{array}$ & $\begin{array}{r}19637_{21 / 2}-54137_{11 / 2}^{\circ} \\
23234_{41 / 2}-57729_{31 / 2}^{\circ} \\
a^{4} \mathrm{G}_{41 / 2}-51045_{31 / 2}^{\circ} \\
20039_{31 / 2}-54498_{31 / 2}^{\circ} \\
a^{4} \mathrm{G}_{31 / 2}-51045_{31 / 2}^{\circ}\end{array}$ & \\
\hline $\begin{array}{l}\text { 2902. } 201 \\
2903.500 \\
2904.081 \\
2904.556 \\
2904.843\end{array}$ & $\begin{array}{l}30 A \\
8 \\
2 \\
-?\end{array}$ & $\begin{array}{r}20 \\
50 \\
80 \\
8 \\
5\end{array}$ & $\begin{array}{l}34446.52 \\
34431.11 \\
34424.22 \\
34418.58 \\
34415.18\end{array}$ & $\begin{array}{l}-0.12 \\
-0.09 \\
-0.01 \\
-0.08 \\
-0.08\end{array}$ & $\begin{array}{r}29341_{41 / 2}-63788_{31 / 2}^{\circ} \\
26929_{51 / 2}-61360_{41 / 2}^{\circ} \\
20534_{51 / 2}-54958_{51 / 2}^{\circ} \\
22194_{31 / 2}-56612_{31 / 2}^{\circ} \\
a^{4} \mathrm{D}_{01 / 2}-47588_{11 / 2}^{\circ}\end{array}$ & $\begin{array}{l}6 \\
6\end{array}$ \\
\hline $\begin{array}{l}\text { 2905. } 602 \\
2906.416 \\
2908.504 \\
2910.332 \\
2911.542\end{array}$ & $\begin{array}{l}8 A \\
2 \\
3 \\
2 \\
2\end{array}$ & $\begin{array}{l}12 \\
12 \\
25 \\
10 \\
20\end{array}$ & $\begin{array}{l}34406.20 \\
34396.57 \\
34371.87 \\
34350.30 \\
34336.01\end{array}$ & $\begin{array}{r}0.00 \\
+0.10 \\
-0.06 \\
-0.02 \\
-0.07 \\
-0.07\end{array}$ & $\begin{array}{l}23450_{21 / 2}-57856_{21 / 2}^{\circ} \\
22535_{01 / 2}-56932_{11 / 2}^{\circ} \\
22503_{11 / 2}-56874_{211 / 2} \\
22194_{31 / 2}-56544_{21 / 2}^{\circ} \\
20039_{31 / 2}-54375_{21 / 2}^{\circ} \\
30633_{41 / 2}-64969_{51 / 2}^{\circ}\end{array}$ & 6 \\
\hline $\begin{array}{l}2912.464 \\
2912.584 \\
2912.986 \\
2913.614 \\
2913.748\end{array}$ & $\begin{array}{r}2 \\
3 \\
1 \\
12\end{array}$ & $\begin{array}{r}25 \\
5 \\
\\
2 \\
30\end{array}$ & $\begin{array}{l}34325.15 \\
34323.73 \\
34319.00 \\
34311.60 \\
34310.02\end{array}$ & $\begin{array}{l}-0.09 \\
-0.11 \\
+0.13 \\
+0.11\end{array}$ & $\begin{array}{r}a^{4} \mathrm{~F}_{41 / 2}-49181_{41 / 2}^{\circ} \\
a^{4} \mathrm{P}_{11 / 2}-44911_{11 / 2}^{\circ} \\
26929_{51 / 2}-61240_{51 / 2}^{\circ} \\
a^{4} \mathrm{G}_{41 / 2}-50863^{\circ}{ }^{\circ 1 / 2}\end{array}$ & 6 \\
\hline $\begin{array}{l}\text { 2914. } 272 \\
2914.655 \\
2916.347 \\
2916.584 \\
2916.768\end{array}$ & $\begin{array}{l}3 \\
3\end{array}$ & $\begin{array}{r}30 \\
15 \\
5 \\
8 \\
10\end{array}$ & $\begin{array}{l}34303.85 \\
34299.34 \\
34279.44 \\
34276.66 \\
34274.50\end{array}$ & $\begin{array}{l}-0.28 \\
+0.05 \\
+0.02 \\
-0.08 \\
+0.22 \\
-0.08\end{array}$ & $\begin{array}{r}20455_{11 / 2}-54760_{01 / 2}^{\circ} \\
19070_{41 / 2}-53369_{41 / 2}^{\circ} \\
23450_{21 / 2}-57729_{31 / 2}^{\circ} \\
27273_{31 / 2}-61550_{31 / 2}^{\circ} \\
a^{4} \mathrm{D}_{21 / 2}-49242_{21 / 2}^{\circ} \\
18000_{31 / 2}-52275_{31 / 2}^{\circ}\end{array}$ & 6 \\
\hline $\begin{array}{l}2916.896 \\
2917.389 \\
2917.540 \\
2917.895 \\
2918.633\end{array}$ & $\begin{array}{r}2 \\
3 \\
1 \\
25\end{array}$ & $\begin{array}{r}12 \\
5 \\
5 \\
3 \\
100\end{array}$ & $\begin{array}{l}34273.00 \\
34267.20 \\
34265.42 \\
34261.25 \\
34252.58\end{array}$ & $\begin{array}{l}-0.38 \\
-0.10 \\
+0.02 \\
-0.25 \\
-0.04\end{array}$ & $\begin{array}{r}a^{4} \mathrm{G}_{31 / 2}-50863_{41 / 2}^{\circ} \\
a^{4} \mathrm{~F}_{41 / 2}-49124_{431 / 2}^{\circ} \\
26158_{41 / 2}-60424_{31 / 2}^{\circ} \\
25672_{21 / 2}^{\circ}-59933_{31 / 2}^{\circ} \\
a^{4} \mathrm{~F}_{21 / 2}-45553_{11 / 2}^{\circ}\end{array}$ & 7 \\
\hline $\begin{array}{l}2918.976 \\
2919.508 \\
2920.903 \\
2921.908 \\
2922.674\end{array}$ & $\begin{array}{l}2 \\
2 \\
-10 A \\
-\end{array}$ & $\begin{array}{r}10 \\
12 \\
6 \\
12 \\
5\end{array}$ & $\begin{array}{l}34248.57 \\
34242.33 \\
34225.98 \\
34214.21 \\
34205.24\end{array}$ & $\begin{array}{l}-0.11 \\
-0.15 \\
+0.04 \\
-0.09 \\
+0.04\end{array}$ & $\begin{array}{l}20455_{11 / 2}-54704_{21 / 2}^{\circ} \\
20780_{41 / 2}-55022_{31 / 2}^{\circ} \\
31100_{51 / 2}-65326_{51 / 2}^{\circ} \\
28118_{21 / 2}-62333_{21 / 2}^{\circ} \\
23046_{31 / 2}-57252_{21 / 2}^{\circ}\end{array}$ & $d$ \\
\hline $\begin{array}{l}\text { 2923. } 382 \\
2923.451 \\
2923.980 \\
2924.996 \\
2925.834\end{array}$ & $\begin{array}{l}5 A \\
1 \\
3 \\
4 \\
5\end{array}$ & $\begin{array}{r}10 \\
10 \\
12 \\
40 \\
125\end{array}$ & $\begin{array}{l}34196.95 \\
34196.15 \\
34189.96 \\
34178.08 \\
34168.30\end{array}$ & $\begin{array}{r}-0.03 \\
+0.04 \\
-0.10 \\
-0.12 \\
-0.04\end{array}$ & $\begin{array}{r}25672_{21 / 2}-59869_{31 / 2}^{\circ} \\
a^{4} \mathrm{G}_{21 / 2}-50430_{11 / 2}^{\circ} \\
25209_{41 / 2}-59399_{41 / 2}^{\circ} \\
20780_{41 / 2}-54958_{51 / 2}^{\circ} \\
33910_{51 / 2}-68078_{61 / 2}^{\circ}\end{array}$ & $\begin{array}{l}6 \\
5 \\
5\end{array}$ \\
\hline $\begin{array}{l}2926.836 \\
2926.990 \\
2927.710 \\
2929.984 \\
2931.530\end{array}$ & $\begin{array}{c}3 \\
40 A \\
3 \\
3 \\
2\end{array}$ & $\begin{array}{l}15 \\
10 \\
20 \\
20 \\
20\end{array}$ & $\begin{array}{l}34156.60 \\
34154.80 \\
34146.40 \\
34119.90 \\
34101.90\end{array}$ & $\begin{array}{r}-0.10 \\
+0.26 \\
-0.06 \\
-0.07 \\
-0.04\end{array}$ & $\begin{array}{r}a^{4} \mathrm{D}_{21 / 2}-49124{ }^{\circ} 1 / 2 \\
a^{4} \mathrm{P}_{21 / 2}-47588_{11 / 2}^{\circ} \\
19276_{21 / 2}-53422^{\circ}{ }^{\circ} 1 / 2 \\
26158_{41 / 2}-60278_{411 / 2}^{\circ} \\
33910_{51 / 2}-68012_{51 / 2}^{\circ}\end{array}$ & $\begin{array}{l}\text { res } \\
7 \\
6 \\
6\end{array}$ \\
\hline $\begin{array}{l}\text { 2931. } 895 \\
2932.864 \\
2933.068 \\
2935.201 \\
2935.358\end{array}$ & $\begin{array}{l}5 \\
3 \\
1 \\
2 \\
8\end{array}$ & $\begin{array}{r}35 \\
20 \\
1 \\
6 \\
25\end{array}$ & $\begin{array}{l}34097.65 \\
34086.39 \\
34083.80 \\
34059.25 \\
34057.43\end{array}$ & $\begin{array}{l}-0.06 \\
+0.07 \\
-0.32 \\
+0.01 \\
-0.06\end{array}$ & $\begin{array}{r}26158_{41 / 2}-60256_{31 / 2}^{\circ} \\
18000_{31 / 2}-52087_{21 / 2}^{\circ} \\
28631_{31 / 2}-62715_{41 / 2}^{\circ} \\
28377_{51 / 2}-62437_{41 / 2}^{\circ} \\
a^{4} \mathrm{G}_{21 / 2}-60292_{21 / 2}^{\circ}\end{array}$ & $\begin{array}{l}\text { res } \\
\text { res }\end{array}$ \\
\hline $\begin{array}{l}\text { 2935. } 726 \\
2935.806 \\
\text { 2936. } 61 \\
2937.200 \\
2937.61\end{array}$ & $\begin{array}{c}\frac{2}{40 A} \\
8 ? \\
10 A\end{array}$ & $\begin{array}{r}10 \\
4 \\
125 \\
15 \\
12\end{array}$ & $\begin{array}{l}34053.16 \\
34052.23 \\
34042.92 \\
34036.07 \\
34031.33\end{array}$ & $\begin{array}{l}-0.03 \\
-0.20 \\
+0.26 \\
-0.02 \\
-0.19\end{array}$ & $\begin{array}{l}19276_{21 / 2}-53329_{11 / 2}^{\circ} \\
27273_{31 / 2}-61326_{31 / 2}^{\circ} \\
23046_{31 / 2}-57089_{41 / 2}^{\circ} \\
19404_{01 / 2}-5340_{01 / 2}^{\circ} \\
23955_{51 / 2}-57986_{41 / 2}^{\circ}\end{array}$ & 6 \\
\hline
\end{tabular}


Table 3. Classified lines of W II-Continued

\begin{tabular}{|c|c|c|c|c|c|c|}
\hline \multirow{2}{*}{$\begin{array}{l}\text { Wavelength } \\
(\AA)\end{array}$} & \multicolumn{2}{|c|}{ Intensity } & \multicolumn{2}{|c|}{ Wave number $\left(\mathrm{cm}^{-1}\right)$} & \multirow{2}{*}{ Combination } & \multirow{2}{*}{$\begin{array}{l}\text { Zeeman } \\
\text { effect }\end{array}$} \\
\hline & Arc & Spark & Observed & $O-C$ & & \\
\hline \multicolumn{7}{|l|}{ Air } \\
\hline $\begin{array}{l}\text { 2937. } 774 \\
2938.685 \\
2938.872 \\
2939.757 \\
2940.204\end{array}$ & $\begin{array}{l}2 \\
? \\
2 \\
6 \\
8\end{array}$ & $\begin{array}{r}4 \\
4 \\
12 \\
35 \\
60\end{array}$ & $\begin{array}{l}34029.42 \\
34018.87 \\
34016.71 \\
34006.47 \\
34001.30\end{array}$ & $\begin{array}{l}-0.03 \\
-0.03 \\
-0.09 \\
-0.09 \\
-0.07\end{array}$ & $\begin{array}{r}26227_{21 / 2}-60256_{31 / 2}^{\circ} \\
19404_{01 / 2}^{\circ}-53422_{11 / 2}^{\circ} \\
20039_{31 / 2}-54056_{41 / 2}^{\circ} \\
a^{4} \mathrm{D}_{01 / 2}-47179_{11 / 2}^{\circ} \\
a^{4} \mathrm{~F}_{31 / 2}-47413_{21 / 2}^{\circ}\end{array}$ & \\
\hline $\begin{array}{l}\text { 2941. } 476 \\
2942.128 \\
2942.263 \\
2942.618 \\
2945.137\end{array}$ & $\begin{array}{c}4 \\
12 A \\
2 \\
3 \\
-\end{array}$ & $\begin{array}{r}5 \\
10 \\
10 \\
10 \\
6\end{array}$ & $\begin{array}{l}33986.60 \\
33979.07 \\
33977.49 \\
33973.41 \\
33944.35\end{array}$ & $\begin{array}{l}+0.10 \\
-0.16 \\
-0.01 \\
-0.07 \\
+0.02\end{array}$ & $\begin{array}{r}20039_{31 / 2}-54026_{21 / 2}^{\circ} \\
a^{4} \mathrm{P}_{21 / 2}-47413_{21 / 2}^{\circ} \\
a^{4} \mathrm{D}_{31 / 2}-491244^{\circ 1 / 2} \\
a^{4} \mathrm{~F}_{41 / 2}-48830_{31 / 2}^{\circ} \\
22139_{21 / 2}-560844_{11 / 2}^{\circ}\end{array}$ & \\
\hline $\begin{array}{l}2946.454 \\
2947.136 \\
2950.453\end{array}$ & $-?$ & $\begin{array}{r}25 \\
2 \\
40\end{array}$ & $\begin{array}{l}33929.18 \\
33921.33 \\
33883.20\end{array}$ & $\begin{array}{l}-0.15 \\
-0.01\end{array}$ & $\begin{array}{l}34091_{41 / 2}-68012_{51 / 2}^{\circ} \\
24804_{31 / 2}-58687_{41 / 2}^{\circ}\end{array}$ & res \\
\hline 2952. 262 & $75 A$ & 100 & 33862.44 & $\begin{array}{l}-0.22 \\
+0.11\end{array}$ & & $d$ \\
\hline 2952. 974 & $4 A$ & 4 & 33854. 27 & -0.32 & $23234_{41 / 2}-5 \% 089_{41 / 2}^{\circ}$ & \\
\hline $\begin{array}{l}2954.070 \\
2954.482 \\
2955.007 \\
2955.256 \\
2956.67\end{array}$ & $\begin{array}{l}4 A \\
4 \\
8 \\
2 \\
20 A\end{array}$ & $\begin{array}{r}3 \\
15 \\
15 \\
10 \\
10\end{array}$ & $\begin{array}{l}33841.71 \\
33836.99 \\
33830.98 \\
33828.13 \\
33811.96\end{array}$ & $\begin{array}{l}-0.49 \\
-0.01 \\
-0.08 \\
-0.06 \\
-0.08\end{array}$ & $\begin{array}{l}28491_{11 / 2}-62333_{21 / 2}^{\circ} \\
19276_{21 / 2}-53113_{21 / 2}^{\circ} \\
19070_{41 / 2}-52901_{31 / 2}^{\circ} \\
23046_{31 / 2}-56874_{21 / 2}^{\circ} \\
18990_{11 / 2}-52803_{11 / 2}^{\circ}\end{array}$ & 6 res \\
\hline $\begin{array}{l}2957.266 \\
2957.590 \\
2958.972 \\
2959.312 \\
2959.910\end{array}$ & $\begin{array}{r}1 \\
1 \\
- \\
-\end{array}$ & $\begin{array}{r}12 \\
4 \\
2 \\
10\end{array}$ & $\begin{array}{l}33805.12 \\
33801.43 \\
33785.65 \\
33781.77 \\
33774.94\end{array}$ & $\begin{array}{l}-0.06 \\
-0.07 \\
+0.05 \\
-0.17 \\
+0.02\end{array}$ & $\begin{array}{l}28631_{31 / 2}-62437_{41 / 2}^{\circ} \\
23450_{21 / 2}-57252_{21 / 2}^{\circ} \\
19637_{21 / 2}-53422_{11 / 2}^{\circ} \\
27273_{31 / 2}^{\circ}-61055_{41 / 2}^{\circ} \\
26158_{41 / 2}^{\circ}-59933_{31 / 2}^{\circ}\end{array}$ & \\
\hline $\begin{array}{l}\text { 2960. } 771 \\
2961.020 \\
2961.558 \\
2964.412 \\
2964.898\end{array}$ & $\frac{\overline{12}}{\overline{2}}$ & $\begin{array}{r}1 \\
50 \\
2 \\
1 \\
20\end{array}$ & $\begin{array}{l}33765.12 \\
33762.28 \\
33756.15 \\
33723.65 \\
33718.12\end{array}$ & $\begin{array}{l}-0.08 \\
-0.02 \\
-0.23 \\
-0.03 \\
-0.07\end{array}$ & $\begin{array}{r}26227_{21 / 2}-59992_{21 / 2}^{\circ} \\
a^{4} \mathrm{P}_{11 / 2}-44354_{21 / 2}^{\circ} \\
24991_{11 / 2}-58747_{11 / 2}^{\circ} \\
30633_{41 / 2}-64356_{31 / 2}^{\circ} \\
20780_{41 / 2}^{\circ}-54498_{31 / 2}^{\circ}\end{array}$ & res \\
\hline $\begin{array}{l}2965.589 \\
2966.417 \\
2967.926 \\
2970.908 \\
2973.092\end{array}$ & $\begin{array}{l}2 \\
4 A \\
4 \\
2 \\
-\end{array}$ & $\begin{array}{r}15 \\
2 \\
20 \\
5 \\
3\end{array}$ & $\begin{array}{l}33710.27 \\
33700.86 \\
33683.73 \\
33649.92 \\
33625.24\end{array}$ & $\begin{array}{l}-0.13 \\
+0.17 \\
+0.05 \\
-0.20 \\
+0.02\end{array}$ & $\begin{array}{r}26158_{41 / 2}-59869_{31 / 2}^{\circ} \\
19637_{21 / 2}^{\circ}-53338_{31 / 2}^{\circ} \\
a^{4} \mathrm{D}_{31 / 2}-48830_{31 / 2}^{\circ} \\
a^{4} \mathrm{D}_{11 / 2}-48284_{21 / 2}^{\circ} \\
19276_{21 / 2}-52901_{31 / 2}^{\circ}\end{array}$ & 6 \\
\hline $\begin{array}{l}2974.377 \\
2975.078 \\
2976.476 \\
2976.985 \\
2977.442\end{array}$ & $\begin{array}{r}20 \\
3 \\
15 \\
-\end{array}$ & $\begin{array}{r}35 \\
10 \\
50 \\
2 \\
3\end{array}$ & $\begin{array}{l}33610.67 \\
33602.75 \\
33586.97 \\
33581.23 \\
33576.08\end{array}$ & $\begin{array}{l}+0.12 \\
-0.01 \\
+0.03 \\
-0.01 \\
-0.02\end{array}$ & $\begin{array}{r}a^{4} \mathrm{~F}_{21 / 2}-44911_{11 / 2}^{\circ} \\
18990_{11 / 2}-52593_{01 / 2}^{\circ} \\
a^{4} \mathrm{~F}_{11 / 2}-42298_{11 / 2}^{\circ} \\
22503_{11 / 2}-56084{ }^{\circ} 1 \frac{1}{2} \\
a^{4} \mathrm{~F}_{21 / 2}-z^{6} \mathrm{~F}_{31 / 2}^{\circ}\end{array}$ & $\begin{array}{l}\text { res } \\
\text { res }\end{array}$ \\
\hline $\begin{array}{l}2977.580 \\
2977.950 \\
2978.332 \\
2981.319 \\
2982.219\end{array}$ & $\begin{array}{l}4 \\
3 \\
1 \\
1 \\
2\end{array}$ & $\begin{array}{r}40 \\
20 \\
6 \\
\\
25\end{array}$ & $\begin{array}{l}33574.52 \\
33570.35 \\
33566.05 \\
33532.41 \\
33522.29\end{array}$ & $\begin{array}{l}+0.04 \\
+0.04 \\
+0.11 \\
+0.10 \\
+0.10\end{array}$ & $\begin{array}{l}30633_{11 / 2}-64207_{41 / 2}^{\circ} \\
20445_{11 / 2}-54026_{21 / 2}^{\circ} \\
23046_{31 / 2}-56612_{31 / 2}^{\circ} \\
24804_{31 / 2}-58396_{21 / 2}^{\circ} \\
20534_{51 / 2}-54056_{41 / 2}^{\circ}\end{array}$ & 6 \\
\hline $\begin{array}{l}\text { 2984. } 426 \\
2985.830 \\
2986.103 \\
\text { 2986. } 382 \\
2987.294\end{array}$ & $\begin{array}{r}5 \\
3 \\
15\end{array}$ & $\begin{array}{r}6 \\
6 \\
25 \\
20 \\
60\end{array}$ & $\begin{array}{l}33497.50 \\
33481.75 \\
33478.69 \\
33475.57 \\
33465.35\end{array}$ & $\begin{array}{l}-0.10 \\
-0.02 \\
+0.09 \\
+0.06 \\
-0.19\end{array}$ & $\begin{array}{r}23046_{31 / 2}-56544^{\circ} 2_{1 / 2} \\
23450_{21 / 2}-56932_{11 / 2}^{\circ} \\
25209_{41 / 2}-58687^{\circ}{ }^{\circ 1 / 2} \\
a^{4} \mathrm{~F}_{41 / 2}-48332^{\circ}{ }^{1 / 2} \\
a^{4} \mathrm{P}_{01 / 2}-42298_{11 / 2}^{\circ}\end{array}$ & $\begin{array}{l}6 \\
\text { res } \\
\text { res }\end{array}$ \\
\hline $\begin{array}{l}\text { 2988. } 502 \\
2988.771 \\
2990.850 \\
2991.470 \\
2991.855\end{array}$ & 4 & $\begin{array}{r}12 \\
12 \\
12 \\
4 \\
3\end{array}$ & $\begin{array}{l}33451.82 \\
33448.81 \\
33425.56 \\
33418.63 \\
33414.33\end{array}$ & $\begin{array}{l}-0.07 \\
+0.13 \\
-0.47 \\
-0.25 \\
-0.25\end{array}$ & $\begin{array}{c}a^{4} \mathrm{D}_{01 / 2}-46625_{01 / 2}^{\circ} \\
20780_{41 / 2}-54229_{51 / 2}^{\circ} \\
a^{4} \mathrm{G}_{51 / 2}-50863_{11 / 2}^{\circ} \\
24918_{11 / 2}-58336_{21 / 2}^{\circ} \\
28187_{61 / 2}-61602_{61 / 2}^{\circ}\end{array}$ & res \\
\hline
\end{tabular}


TABle 3. Classified lines of $\mathrm{W} \mathrm{I}-$-Continued

\begin{tabular}{|c|c|c|c|c|c|c|}
\hline \multirow{2}{*}{$\begin{array}{l}\text { Wavelength } \\
(\AA)\end{array}$} & \multicolumn{2}{|c|}{ Intensity } & \multicolumn{2}{|c|}{ Wave number $\left(\mathrm{cm}^{-1}\right)$} & \multirow{2}{*}{ Combination } & \multirow{2}{*}{$\begin{array}{c}\text { Zeeman } \\
\text { effect }\end{array}$} \\
\hline & Arc & Spark & Observed & $O-C$ & & \\
\hline Air & & & & & & \\
\hline $\begin{array}{l}\text { 2992. } 043 \\
2994.700 \\
2995.129 \\
2997.685 \\
2998.050\end{array}$ & 3 & $\begin{array}{r}18 \\
60 \\
6 \\
8 \\
5\end{array}$ & $\begin{array}{l}33412.23 \\
33382.59 \\
33377.81 \\
33349.35 \\
33345.29\end{array}$ & $\begin{array}{l}-0.06 \\
-0.06 \\
-0.02 \\
-0.13\end{array}$ & $\begin{array}{l}27273_{31 / 2}-60656_{21 / 2}^{\circ} \\
23234_{41 / 2}-56612_{31 / 2}^{\circ} \\
26929_{51 / 2}-60278_{41 / 2}^{\circ} \\
24991_{11 / 2}-58396_{21 / 2}^{\circ}\end{array}$ & \\
\hline $\begin{array}{l}\text { 2998. } 693 \\
3000.499 \\
3000.624 \\
3002.287 \\
3003.070\end{array}$ & $\begin{array}{r}15 \\
5 \\
2 \\
4\end{array}$ & $\begin{array}{r}50 \\
3 \\
50 \\
40 \\
10\end{array}$ & $\begin{array}{l}33338.14 \\
33318.07 \\
33316.69 \\
33298.23 \\
33289.55\end{array}$ & $\begin{array}{l}-0.05 \\
-0.04 \\
+0.03 \\
-0.10 \\
+0.05\end{array}$ & $\begin{array}{c}a^{4} \mathrm{~F}_{11 / 2}-z^{6} \mathrm{~F}_{21 / 2}^{\circ} \\
23450_{21 / 2}-56768_{31 / 2}^{\circ} \\
a^{4} \mathrm{D}_{21 / 2}-48284^{\circ}{ }^{\circ 1 / 2} \\
20039_{31 / 2}-53938_{31 / 2}^{\circ} \\
26929_{51 / 2}-60218_{51 / 2}^{\circ}\end{array}$ & $\begin{array}{l}\text { res } \\
\text { res } \\
6 \text { res } \\
6\end{array}$ \\
\hline $\begin{array}{l}3003.435 \\
3004.284 \\
3007.511 \\
3008.946 \\
3010.131\end{array}$ & 2 & $\begin{array}{c}15 \\
5 \\
4 ? \\
25 \\
3\end{array}$ & $\begin{array}{l}33285.51 \\
33276.10 \\
33240.40 \\
33224.55 \\
33211.47\end{array}$ & $\begin{array}{l}-0.11 \\
-0.06 \\
-0.20 \\
+0.17 \\
-0.19\end{array}$ & $\begin{array}{l}23803_{31 / 2}-57089_{41 / 2}^{\circ} \\
20780_{41 / 2}-54056_{41 / 2}^{\circ} \\
26158_{41 / 2}-59399_{41 / 2}^{\circ} \\
28377_{51 / 2}-61602_{61 / 2}^{\circ} \\
28377_{51 / 2}^{\circ}-61589_{51 / 2}^{\circ}\end{array}$ & 7 \\
\hline $\begin{array}{l}3010.750 \\
3011.102 \\
3011.327 \\
3012.102 \\
3013.093\end{array}$ & 4 & $\begin{array}{r}80 \\
2 \\
4 \\
15 \\
1\end{array}$ & $\begin{array}{l}33204.64 \\
33200.76 \\
33198.27 \\
33189.74 \\
33178.82\end{array}$ & $\begin{array}{l}+0.04 \\
-0.05 \\
-0.02 \\
+0.10 \\
+0.05\end{array}$ & $\begin{array}{l}19070_{41 / 2}-5227_{5}^{\circ}{ }_{31 / 2}^{1} \\
27273_{31 / 2}-6047_{421 / 2}^{\circ} \\
22194_{31 / 2}-55392_{41 / 2}^{\circ} \\
19404_{01 / 2}-52593_{01 / 2}^{\circ} \\
23234_{41 / 2}-56413_{41 / 2}^{\circ}\end{array}$ & res \\
\hline $\begin{array}{l}3014.200 \\
3014.606 \\
3015.694 \\
3017.148 \\
3018.624\end{array}$ & $\begin{array}{l}6 A \\
2 \\
1 \\
2 \\
1\end{array}$ & $\begin{array}{r}3 \\
25 \\
8 \\
25 \\
20\end{array}$ & $\begin{array}{l}33166.63 \\
33162.17 \\
33150.20 \\
33134.23 \\
33118.03\end{array}$ & $\begin{array}{l}-0.48 \\
-0.07 \\
-0.08 \\
+0.17 \\
-0.04\end{array}$ & $\begin{array}{l}25169_{11 / 2}-58336_{21 / 2}^{\circ} \\
23450_{21 / 2}-56612_{31 / 2}^{\circ} \\
27273_{31 / 2}-60424_{31 / 2}^{\circ} \\
23955_{51 / 2}-57089_{41 / 2}^{\circ} \\
26158_{41 / 2}-5926_{31 / 2}^{\circ}\end{array}$ & $\begin{array}{l}\text { res } \\
6 \\
5\end{array}$ \\
\hline $\begin{array}{l}3019.628 \\
3020.630 \\
3021.982 \\
3022.482 \\
3022.672\end{array}$ & $\begin{array}{r}4 \\
20\end{array}$ & $\begin{array}{r}3 \\
20 \\
80 \\
20 \\
30\end{array}$ & $\begin{array}{l}33107.08 \\
33096.04 \\
33081.23 \\
33075.76 \\
33073.68\end{array}$ & $\begin{array}{l}+0.04 \\
-0.02 \\
-0.24 \\
-0.02 \\
+0.06 \\
-0.10\end{array}$ & $\begin{array}{c}31100_{51 / 2}-64207_{41 / 2}^{\circ}{ }_{41 / 2} \\
18990_{11 / 2}-52087^{\circ}{ }^{\circ}{ }_{1 / 2} \\
a^{4} \mathrm{~F}_{31 / 2}-z^{6} \mathrm{~F}_{41 / 2}^{\circ} \\
25672_{21 / 2}-587_{4} 7_{11 / 2}^{\circ} \\
28491_{11 / 2}-61566_{21 / 2}^{\circ} \\
20039_{31 / 2}-53113_{21 / 2}^{\circ}\end{array}$ & \\
\hline $\begin{array}{l}3022.906 \\
3024.502 \\
3025.993 \\
3027.369 \\
3028.750\end{array}$ & $\begin{array}{r}2 \\
25\end{array}$ & $\begin{array}{r}6 \\
250 \\
1 \\
6 \\
10\end{array}$ & $\begin{array}{l}33071.12 \\
33053.67 \\
33037.38 \\
33022.33 \\
33007.31\end{array}$ & $\begin{array}{r}-0.03 \\
-0.07 \\
-0.02 \\
0.00 \\
+0.05\end{array}$ & $\begin{array}{r}23803_{31 / 2}-56874211 / 2 \\
a^{4} \mathrm{~F}_{21 / 2}-4435421 / 2 \\
25672_{21 / 2}-58709^{\circ} 1 \frac{1}{2} \\
22139_{21 / 2}-55162_{21 / 2}^{\circ} \\
a^{4} \mathrm{G}_{21 / 2}-49242_{21 / 2}^{\circ}\end{array}$ & res \\
\hline $\begin{array}{l}3028.975 \\
3029.524 \\
3030.793 \\
3031.000 \\
3032.450\end{array}$ & 3 & $\begin{array}{r}8 \\
4 \\
2 \\
25 \\
15\end{array}$ & $\begin{array}{l}\text { 33004. } 86 \\
32998.88 \\
32985.06 \\
32982.81 \\
32967.04\end{array}$ & $\begin{array}{l}+0.01 \\
+0.12 \\
+0.11 \\
+0.07 \\
-0.01\end{array}$ & $\begin{array}{l}27273_{31 / 2}-60278_{41 / 2}^{\circ} \\
19276_{21 / 2}-52275_{31 / 2}^{\circ} \\
22503_{11 / 2}-55488_{11 / 2}^{\circ} \\
28377_{51 / 2}-61360_{41 / 2}^{\circ} \\
20455_{11 / 2}-53422_{11 / 2}^{\circ}\end{array}$ & res \\
\hline $\begin{array}{l}3032.660 \\
3033.626 \\
3033.790 \\
3033.913 \\
3036.311\end{array}$ & 4 & $\begin{array}{r}8 \\
30 \\
2 \\
20 \\
1\end{array}$ & $\begin{array}{l}\text { 32964. } 76 \\
32954.26 \\
32952.48 \\
32951.14 \\
32925.11\end{array}$ & $\begin{array}{l}-0.01 \\
-0.02 \\
+0.27 \\
+0.11 \\
-0.14\end{array}$ & $\begin{array}{r}23803_{31 / 2}-56768_{31 / 2}^{\circ} \\
a^{4} \mathrm{D}_{11 / 2}-47588_{11 / 2}^{\circ} \\
22535_{01 / 2}-55488_{11 / 2}^{\circ} \\
19404_{01 / 2}-52355_{01 / 2}^{\circ} \\
24804_{31 / 2}-57729_{31 / 2}^{\circ}\end{array}$ & res \\
\hline $\begin{array}{l}3036.670 \\
3039.578 \\
3044.400 \\
3044.564 \\
3045.216\end{array}$ & $\begin{array}{l}5 \\
3\end{array}$ & $\begin{array}{r}50 \\
20 \\
10 \\
1 \\
15\end{array}$ & $\begin{array}{l}32921.23 \\
32889.73 \\
32837.64 \\
32835.88 \\
32828.85\end{array}$ & $\begin{array}{l}-0.07 \\
+0.05 \\
-0.09 \\
+0.26 \\
+0.07\end{array}$ & $\begin{array}{r}a^{4} \mathrm{P}_{21 / 2}-46355_{21 / 2}^{\circ}-49124^{\circ} \\
a^{4} \mathrm{G}_{21 / 2}-4911 / 2 \\
25169_{11 / 2}-58007_{11 / 2}^{\circ} \\
20534_{51 / 2}-53369_{41 / 2}^{\circ} \\
22194_{31 / 2}-55022_{31 / 2}^{\circ}\end{array}$ & $\begin{array}{l}\text { res } \\
6\end{array}$ \\
\hline $\begin{array}{l}3046.911 \\
3047.060 \\
3048.604 \\
3049.563 \\
3049.850\end{array}$ & $\begin{array}{c}1 \\
4 \\
\frac{5}{5} ?\end{array}$ & $\begin{array}{r}5 \\
15 \\
40 \\
3 \\
50\end{array}$ & $\begin{array}{l}32810.57 \\
32808.97 \\
32792.36 \\
32782.03 \\
32778.96\end{array}$ & $\begin{array}{l}+0.07 \\
+0.07 \\
+0.01 \\
-0.04 \\
-0.01\end{array}$ & $\begin{array}{r}19276_{21 / 2}-52087_{21 / 2}^{\circ} \\
23803_{31 / 2}-56612_{31 / 2}^{\circ} \\
19070_{41 / 2}-51863_{31 / 2}^{\circ} \\
28118_{21 / 2}-60900_{21 / 2}^{\circ} \\
a^{4} \mathrm{D}_{11 / 2}-47413_{21 / 2}^{\circ}\end{array}$ & $\begin{array}{l}6 \\
\text { res }\end{array}$ \\
\hline
\end{tabular}


TABLE 3. Classified lines of W II-Continued

\begin{tabular}{|c|c|c|c|c|c|c|}
\hline \multirow{2}{*}{$\begin{array}{l}\text { Wavelength } \\
(\AA)\end{array}$} & \multicolumn{2}{|c|}{ Intensity } & \multicolumn{2}{|c|}{ Wave number $\left(\mathrm{cm}^{-1}\right)$} & \multirow{2}{*}{ Combination } & \multirow{2}{*}{$\begin{array}{l}\text { Zeeman } \\
\text { effect }\end{array}$} \\
\hline & Arc & Spark & Observed & $O-C$ & & \\
\hline \multicolumn{7}{|l|}{ Air } \\
\hline $\begin{array}{l}3051.29 \\
3053.354 \\
3053.420 \\
3054.129 \\
3058.452\end{array}$ & $\begin{array}{r}25 \\
6\end{array}$ & $\left.\begin{array}{c}250 \\
? \\
20 \\
1 \\
20\end{array}\right\}$ & $\begin{array}{l}32763.49 \\
32741.34 \\
32740.64 \\
32733.03 \\
32686.79\end{array}$ & $\begin{array}{l}+0.03 \\
+0.02 \\
+0.08 \\
+0.03 \\
-0.04\end{array}$ & $\begin{array}{r}a^{4} \mathrm{~F}_{31 / 2}-46175_{31 / 2}^{\circ} \\
a^{4} \mathrm{P}_{21 / 2}-46175_{31 / 2}^{\circ} \\
23803_{31 / 2}-56544_{21 / 2}^{\circ} \\
26158_{41 / 2}-58891_{51 / 2}^{112} \\
25169_{11 / 2}-57856_{21 / 2}^{\circ}\end{array}$ & res \\
\hline $\begin{array}{l}3059.268 \\
3061.027 \\
3061.678 \\
3063.422 \\
3063.972\end{array}$ & $\begin{array}{l}6 \\
4\end{array}$ & $\begin{array}{r}3 \\
10 \\
15 \\
8 \\
30\end{array}$ & $\begin{array}{l}32678.06 \\
32659.28 \\
32652.35 \\
32633.74 \\
32627.89\end{array}$ & $\begin{array}{l}+0.06 \\
+0.04 \\
-0.08 \\
-0.06 \\
-0.01\end{array}$ & $\begin{array}{r}28377_{51 / 2}-61055_{41 / 2}^{\circ} \\
22503_{11 / 2}-55162_{21 / 2}^{\circ} \\
a^{4} \mathrm{G}_{31 / 2}-49242_{21 / 2}^{\circ} \\
23450_{21 / 2}-56084_{11 / 2}^{11} \\
a^{4} \mathrm{G}_{41 / 2}-49181_{41 / 2}^{\circ}\end{array}$ & $\begin{array}{l}\text { res } \\
\text { res }\end{array}$ \\
\hline $\begin{array}{l}3064.635 \\
3066.976 \\
3067.410 \\
3067.572 \\
3067.856\end{array}$ & $\begin{array}{l}6 \\
3 \\
5 \\
6\end{array}$ & $\begin{array}{r}3 \\
60 \\
6 \\
50 \\
60\end{array}$ & $\begin{array}{l}32620.83 \\
32595.93 \\
32591.32 \\
32589.61 \\
32586.59\end{array}$ & $\begin{array}{l}+0.01 \\
+0.07 \\
-0.05 \\
+0.02 \\
+0.08\end{array}$ & 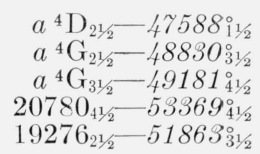 & $\begin{array}{l}7 \\
6 \\
7\end{array}$ \\
\hline $\begin{array}{l}\text { 3069. } 288 \\
3071.234 \\
3071.730 \\
\text { 3072. } 740 \\
\text { 3073. } 403\end{array}$ & $\begin{array}{l}8 \\
1 \\
5 \\
4\end{array}$ & $\begin{array}{r}80 \\
15 \\
50 \\
30 \\
4\end{array}$ & $\begin{array}{l}32571.38 \\
32550.74 \\
32545.48 \\
32534.78 \\
32527.77\end{array}$ & $\begin{array}{r}0.00 \\
-0.08 \\
-0.10 \\
-0.07 \\
+0.36\end{array}$ & $\begin{array}{r}a^{4} \mathrm{G}_{41 / 2}-49124^{\circ}{ }^{1 / 2} \\
26158_{41 / 2}-58709^{\circ}{ }^{1 / 2} \\
a^{4} \mathrm{D}_{11 / 2}-47179_{11 / 2}^{\circ} \\
a^{4} \mathrm{G}_{31 / 2}-49124^{\circ}{ }^{11 / 2} \\
20039_{31 / 2}-5256^{7} 7_{41 / 2}^{\circ}\end{array}$ & $\begin{array}{l}\text { res } \\
\text { res } \\
\text { res } \\
\text { res }\end{array}$ \\
\hline $\begin{array}{l}3075.963 \\
3077.520 \\
3078.868 \\
3079.992 \\
3080.787\end{array}$ & $\begin{array}{r}30 \\
?\end{array}$ & $\begin{array}{r}8 \\
300 \\
15 \\
20 \\
2\end{array}$ & $\begin{array}{l}32500.70 \\
32484.26 \\
32470.03 \\
32458.19 \\
32449.81\end{array}$ & $\begin{array}{l}+0.06 \\
+0.03 \\
-0.05 \\
+0.17\end{array}$ & $\begin{array}{l}23955_{51 / 2}-56439_{61 / 2}^{\circ} \\
26929_{51 / 2}-59399_{41 / 2}^{\circ} \\
23955_{51 / 2}-56413_{41 / 2}^{\circ} \\
19637_{21 / 2}-52087_{21 / 2}^{\circ}\end{array}$ & $\begin{array}{l}5 \\
\text { res } \\
4\end{array}$ \\
\hline $\begin{array}{l}3081.042 \\
3081.215 \\
3083.520 \\
3087.394 \\
3089.742\end{array}$ & $\begin{array}{r}4 \\
1 \\
2 \\
10\end{array}$ & $\begin{array}{r}40 \\
3 \\
20 \\
50 \\
6\end{array}$ & $\begin{array}{l}32447.13 \\
32445.30 \\
32421.05 \\
32380.37 \\
32355.77\end{array}$ & $\begin{array}{r}+0.06 \\
-0.21 \\
0.00 \\
+0.05 \\
0.00\end{array}$ & $\begin{array}{r}18990_{11 / 2}-51438_{21 / 2}^{\circ} \\
a^{4} \mathrm{D}_{21 / 2}-47413_{21 / 2}^{\circ} \\
23955_{51 / 2}^{\circ}-56376_{51 / 2}^{\circ} \\
a^{4} \mathrm{D}_{01 / 2}^{\circ}-45553_{11 / 2}^{\circ} \\
28118_{21 / 2}^{\circ} 60474^{\circ}{ }^{\circ 1 / 2}\end{array}$ & $\begin{array}{l}\text { res } \\
6 \\
\text { res }\end{array}$ \\
\hline $\begin{array}{l}\text { 3090. } 712 \\
\text { 3091. } 870 \\
3094.629 \\
\text { 3095. } 870 \\
\text { 3098. } 031\end{array}$ & 2 & $\begin{array}{r}2 \\
3 \\
4 \\
50 \\
2\end{array}$ & $\begin{array}{l}32345.62 \\
32333.49 \\
32304.67 \\
32291.73 \\
32269.20\end{array}$ & $\begin{array}{l}+0.07 \\
+0.01 \\
+0.18 \\
+0.10 \\
+0.09\end{array}$ & $\begin{array}{l}23046_{31 / 2}-55392_{41 / 2}^{\circ} \\
30633_{41 / 2}-62966_{51 / 2}^{\circ} \\
22194_{31 / 2}-54498_{31 / 2}^{\circ} \\
18000_{31 / 2}-50292_{21 / 2}^{\circ} \\
28631_{31 / 2}-60900_{21 / 2}^{\circ}\end{array}$ & res \\
\hline $\begin{array}{l}3098.300 \\
3098.580 \\
3098.870 \\
3100.074 \\
3100.736\end{array}$ & $\frac{2}{-}$ & $\begin{array}{r}12 \\
12 \\
8 \\
5 \\
50\end{array}$ & $\begin{array}{l}32266.40 \\
32263.48 \\
32260.47 \\
32247.93 \\
32241.05\end{array}$ & $\begin{array}{l}+0.09 \\
+0.04 \\
+0.03 \\
+0.03 \\
+0.02\end{array}$ & $\begin{array}{r}a^{4} \mathrm{D}_{31 / 2}-47413_{21 / 2}^{\circ} \\
18990_{11 / 2}-512544^{\circ}{ }^{11 / 2} \\
24991_{11 / 2}-57252_{21 / 2}^{\circ} \\
29341_{41 / 2}-61589^{\circ}{ }^{1 / 2} \\
a^{4} \mathrm{G}_{31 / 2}-48830_{31 / 2}^{\circ}\end{array}$ & res \\
\hline $\begin{array}{l}3101.224 \\
3102.204 \\
3103.517 \\
3104.534 \\
3106.180\end{array}$ & $\begin{array}{l}5 \\
2\end{array}$ & $\begin{array}{r}4 \\
50 \\
12 \\
3 \\
8\end{array}$ & $\begin{array}{l}32235.98 \\
32225.79 \\
32212.16 \\
32201.61 \\
32184.55\end{array}$ & $\begin{array}{l}+0.13 \\
+0.14 \\
+0.04 \\
+0.06 \\
+0.01\end{array}$ & $\begin{array}{r}22139_{21 / 2}-54375_{21 / 2}^{\circ} \\
19637_{21 / 2}-51863_{31 / 2}^{\circ} \\
a^{4} \mathrm{D}_{21 / 2}-47179_{11 / 2}^{\circ} \\
22503_{11 / 2}^{\circ}-547042^{1 / 2} \\
25672_{21 / 2}-57856_{21 / 2}^{\circ}\end{array}$ & res \\
\hline $\begin{array}{l}3106.632 \\
3108.386 \\
3108.778 \\
3110.690 \\
3111.887\end{array}$ & $\begin{array}{l}6 \\
4 \\
1\end{array}$ & $\begin{array}{r}20 \\
3 \\
60 \\
40 \\
4\end{array}$ & $\begin{array}{l}32179.86 \\
32161.71 \\
32157.65 \\
32137.89 \\
32125.52\end{array}$ & $\begin{array}{l}-0.07 \\
+0.20 \\
+0.15 \\
+0.10 \\
+0.04\end{array}$ & $\begin{array}{l}34091_{41 / 2}-66270_{41 / 2}^{\circ} \\
19404_{21 / 2}-51438_{21 / 2}^{\circ} \\
23234_{41 / 2}-55392_{41 / 2}^{\circ} \\
20455_{11 / 2}-52593_{01 / 2}^{\circ} \\
27273_{31 / 2}-59399_{41 / 2}^{\circ}\end{array}$ & $\begin{array}{l}6 \\
\text { res } \\
\text { res }\end{array}$ \\
\hline $\begin{array}{l}3112.463 \\
3112.860 \\
3113.384 \\
3116.100 \\
3117.263\end{array}$ & $\begin{array}{r}1 \\
20\end{array}$ & $\begin{array}{r}4 \\
50 \\
12 \\
3 \\
1\end{array}$ & $\begin{array}{l}32119.58 \\
32115.48 \\
32110.08 \\
32082.09 \\
32070.12\end{array}$ & $\begin{array}{l}-0.02 \\
-0.02 \\
+0.10 \\
-0.04 \\
-0.20\end{array}$ & $\begin{array}{c}a^{4} \mathrm{P}_{21 / 2}-45553_{11 / 2}^{\circ} \\
23046_{31 / 2}-55162^{\circ 11 / 2} \\
26227_{21 / 2}-58337^{21 / 2} \\
25169_{11 / 2}-57252_{21 / 2}^{\circ} \\
24804_{31 / 2}-5684_{421 / 2}^{\circ}\end{array}$ & $\begin{array}{l}\text { res } \\
6\end{array}$ \\
\hline
\end{tabular}


Table 3. Classified lines of W II-Continued

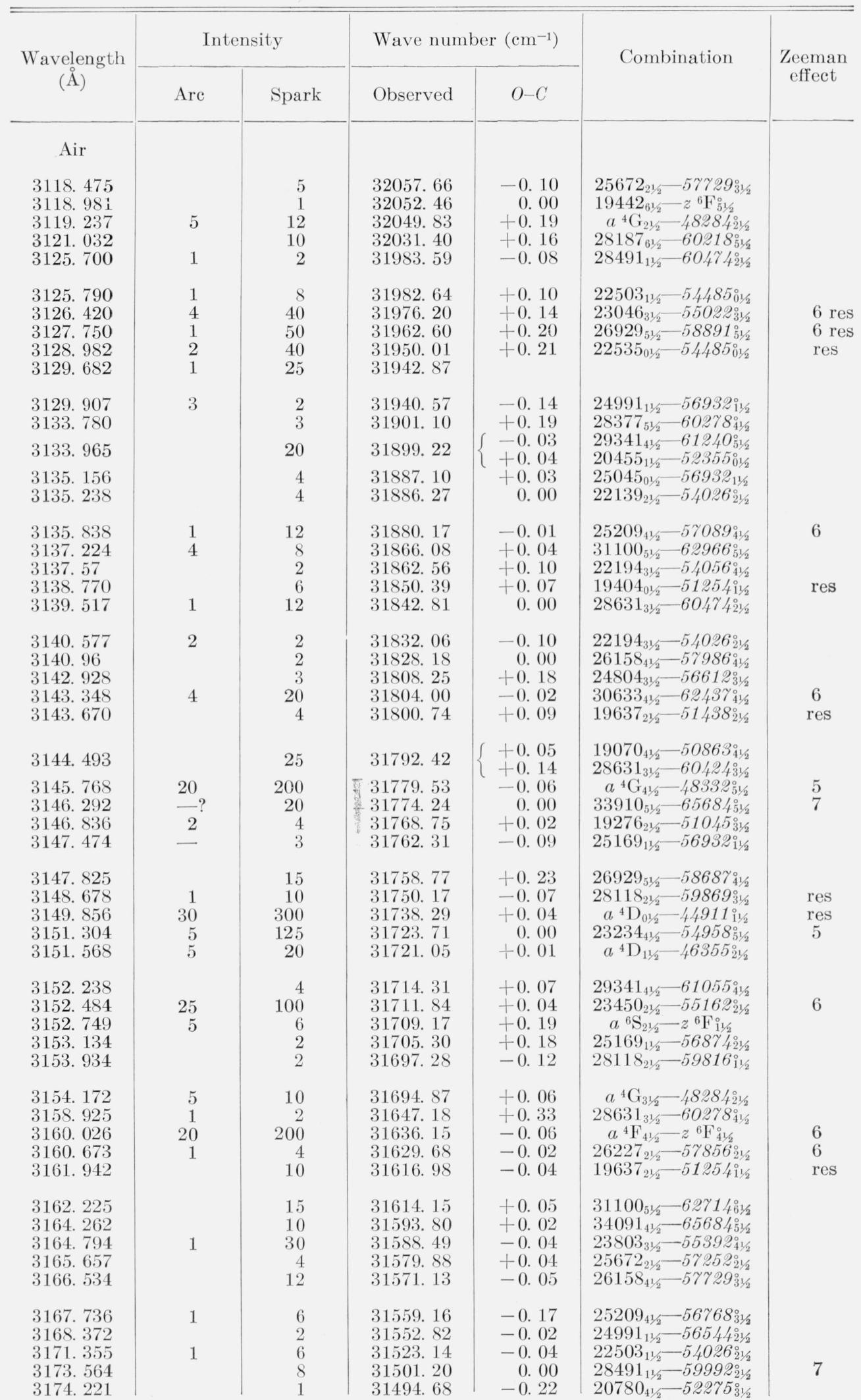


TaBle 3. Classified lines of $\mathrm{W} \mathrm{II}$-Continued

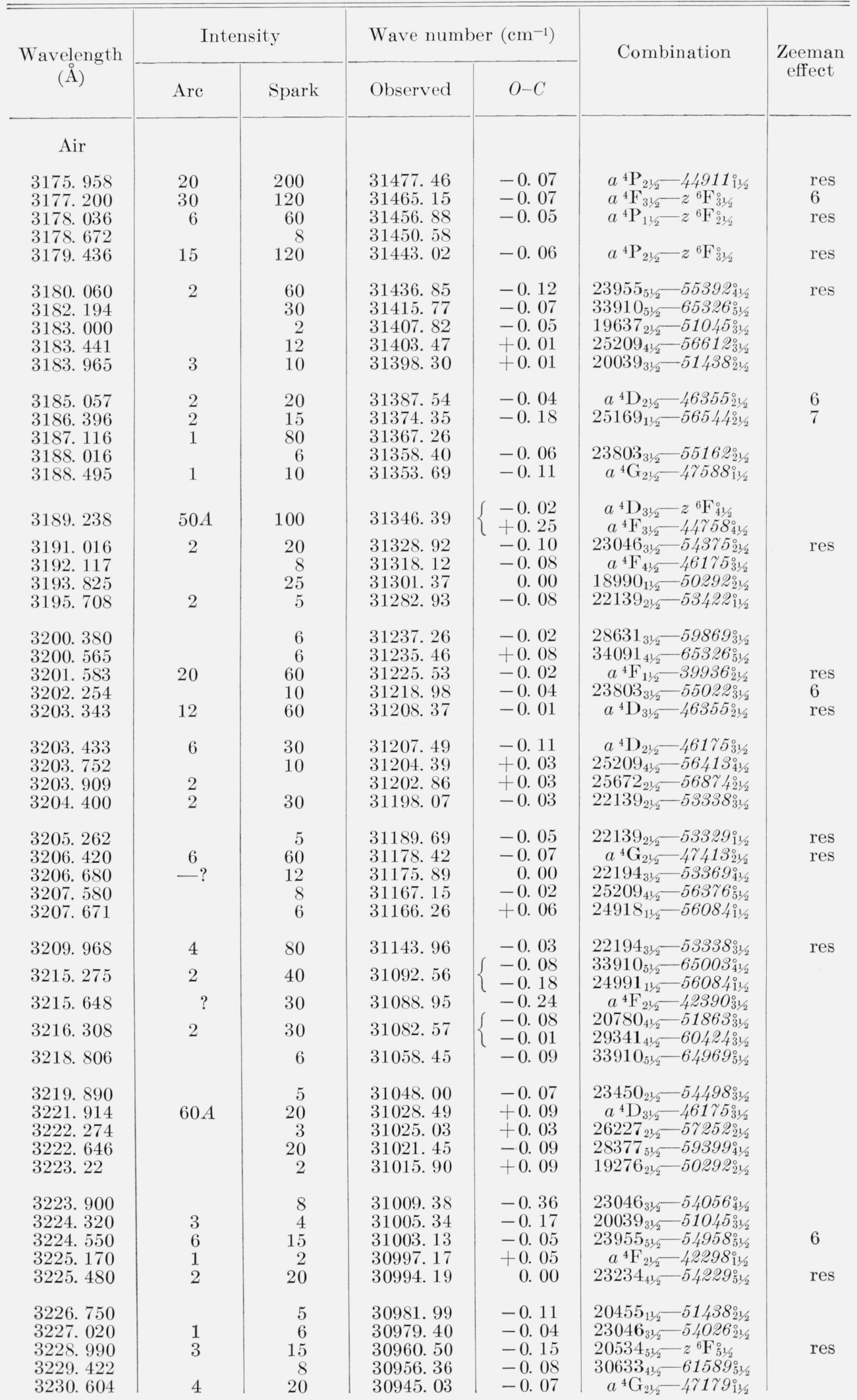


Table 3. Classified lines of $\mathrm{W} \mathrm{II}$-Continued

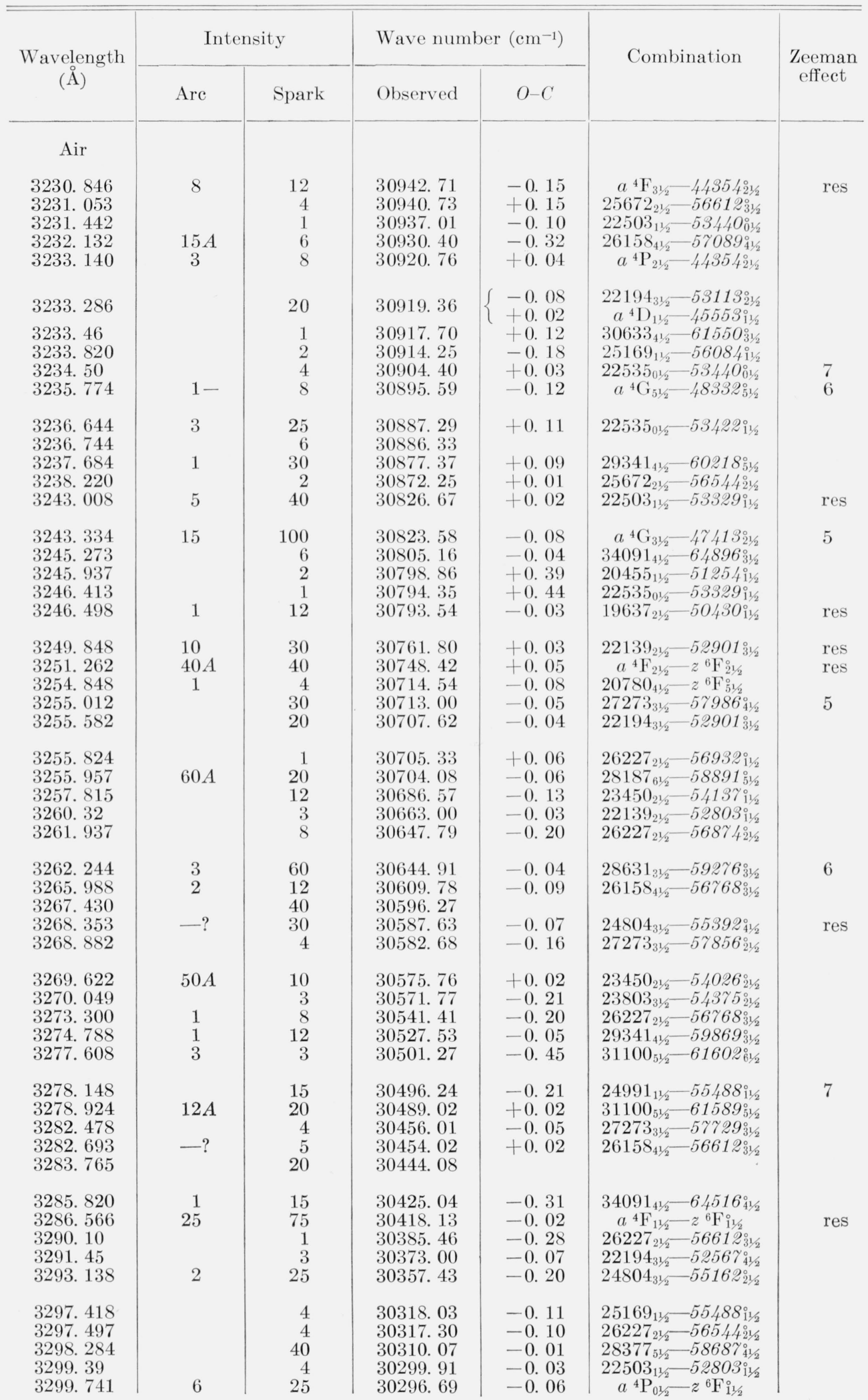


Table 3. Classified lines of $\mathrm{W}$ II-Continued

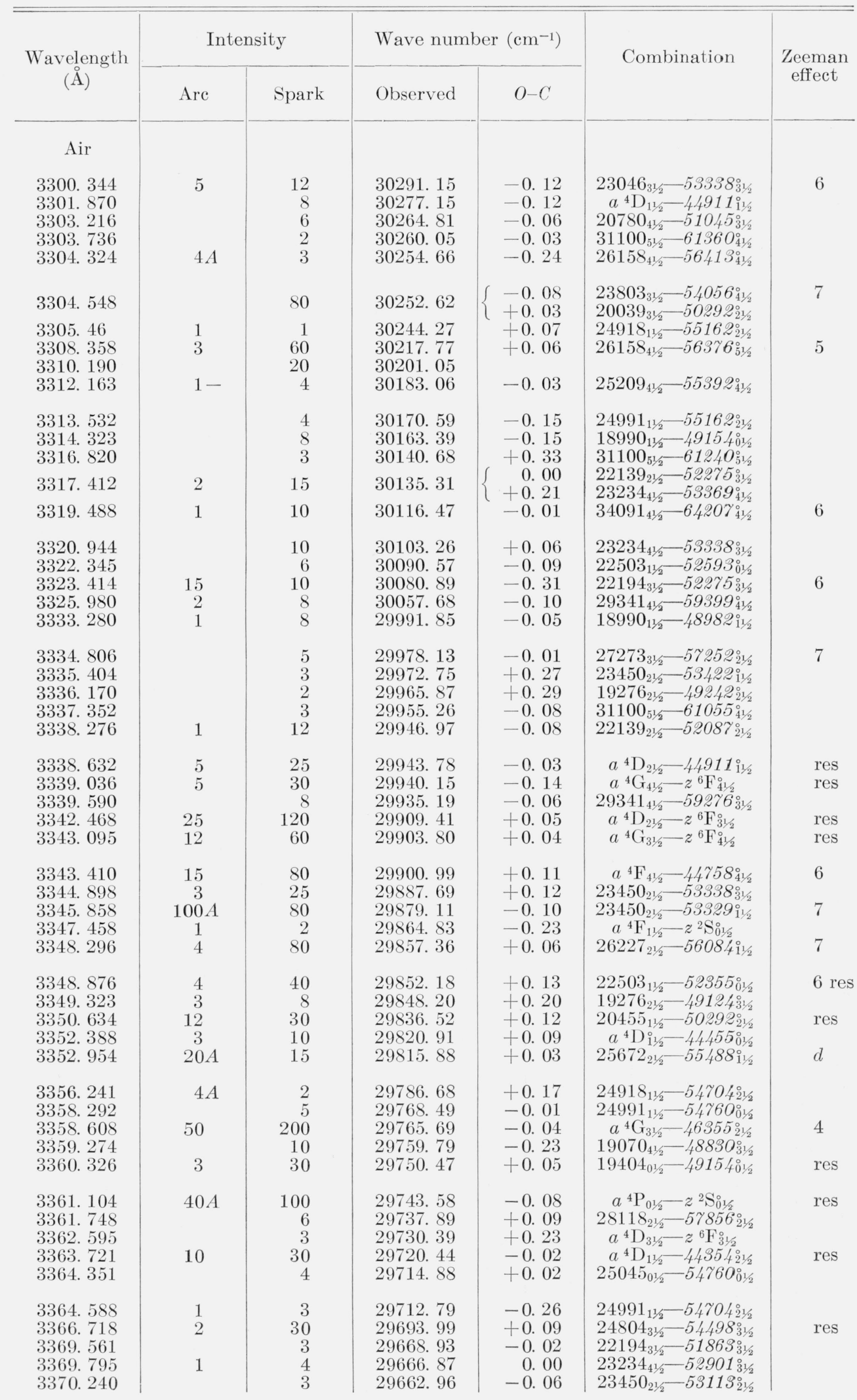


TABLE 3. Classified lines of $\mathrm{W} \mathrm{I}-$ Continued

\begin{tabular}{|c|c|c|c|c|c|c|}
\hline \multirow{2}{*}{$\begin{array}{c}\text { Wavelength } \\
(\AA)\end{array}$} & \multicolumn{2}{|c|}{ Intensity } & \multicolumn{2}{|c|}{ Wave number $\left(\mathrm{cm}^{-1}\right)$} & \multirow{2}{*}{ Combination } & \multirow{2}{*}{$\begin{array}{c}\text { Zeeman } \\
\text { effect }\end{array}$} \\
\hline & Arc & Spark & Observed & $O-C$ & & \\
\hline Air & & & & & & \\
\hline $\begin{array}{l}\text { 3372. } 202 \\
3374.866 \\
3376.146 \\
3379.028 \\
3379.825\end{array}$ & $\begin{array}{r}2 \\
3 \\
30 \\
4\end{array}$ & $\begin{array}{r}15 \\
30 \\
300 \\
40 \\
2\end{array}$ & $\begin{array}{l}29645.70 \\
29622.30 \\
29611.07 \\
29585.81 \\
29578.84\end{array}$ & $\begin{array}{l}+0.01 \\
+0.02 \\
-0.01 \\
+0.06 \\
+0.06\end{array}$ & $\begin{array}{r}30633_{41 / 2}-60278_{41 / 2}^{\circ} \\
a^{4} \mathrm{G}_{41 / 2}-46175_{31 / 2}^{\circ} \\
a^{4} \mathrm{D}_{31 / 2}-44758_{41 / 2}^{\circ} \\
a^{4} \mathrm{G}_{31 / 2}-46175_{31 / 2}^{\circ} \\
19404_{01 / 2}-48982_{11 / 2}^{\circ}\end{array}$ & $\begin{array}{l}6 \\
\text { res } \\
\text { res } \\
\text { res }\end{array}$ \\
\hline $\begin{array}{l}3380.681 \\
3382.598 \\
3383.091 \\
3384.888 \\
3386.502\end{array}$ & $\begin{array}{c}1 \\
50 A \\
2 \\
25 \\
4\end{array}$ & $\begin{array}{r}8 \\
40 \\
50 \\
20 \\
20\end{array}$ & $\begin{array}{l}29571.35 \\
29554.59 \\
29550.28 \\
29534.60 \\
29520.52\end{array}$ & $\begin{array}{l}+0.20 \\
+0.41 \\
+0.10 \\
-0.14 \\
+0.17\end{array}$ & $\begin{array}{l}24804_{31 / 2}-54375_{21 / 2}^{\circ} \\
19276_{21 / 2}-48830_{31 / 2}^{\circ} \\
29341_{41 / 2}-58891_{51 / 2}^{\circ} \\
25169_{11 / 2}-54704_{21 / 2}^{\circ} \\
23046_{31 / 2}-52567_{41 / 2}^{\circ}\end{array}$ & \\
\hline $\begin{array}{l}\text { 3386. } 933 \\
3387.634 \\
3389.544 \\
3389.985 \\
3390.324\end{array}$ & 1 & $\begin{array}{r}3 \\
15 \\
2 \\
1 \\
20\end{array}$ & $\begin{array}{l}29516.76 \\
29510.66 \\
29494.03 \\
29490.19 \\
29487.24\end{array}$ & $\begin{array}{l}+0.16 \\
+0.40 \\
-0.01 \\
+0.05 \\
+0.10\end{array}$ & $\begin{array}{l}28491_{11 / 2}-58007_{11 / 2}^{\circ} \\
26929_{51 / 2}-56439_{61 / 2}^{\circ} \\
24991_{11 / 2}-54485_{01 / 2}^{\circ} \\
25672_{21 / 2}-55162_{21 / 2}^{\circ} \\
19637_{21 / 2}-49124^{\circ}{ }^{1 / 2}\end{array}$ & res \\
\hline $\begin{array}{l}\text { 3394. } 450 \\
3394.907 \\
3395.712 \\
3398.690 \\
3398.922\end{array}$ & $\begin{array}{l}2 \\
1\end{array}$ & $\begin{array}{r}15 \\
3 \\
2 \\
1 \\
120\end{array}$ & $\begin{array}{l}29451.40 \\
29447.44 \\
29440.45 \\
29414.66 \\
29412.65\end{array}$ & $\begin{array}{l}+0.16 \\
+0.33 \\
+0.05 \\
+0.09 \\
+0.02\end{array}$ & $\begin{array}{l}23450_{21 / 2}-52901_{31 / 2}^{\circ} \\
26929_{51 / 2}-56376_{51 / 2}^{\circ} \\
25045_{01 / 2}-54485_{01 / 2}^{\circ} \\
23955_{51 / 2}-53969_{41 / 2}^{\circ} \\
18000_{31 / 2}-47413_{21 / 2}^{\circ}\end{array}$ & 7 \\
\hline $\begin{array}{l}\text { 3401. } 884 \\
3402.194 \\
3404.082 \\
3406.086 \\
3406.588\end{array}$ & $\begin{array}{l}2 \\
1\end{array}$ & $\begin{array}{c}300 \\
40 ? \\
1 \\
12 \\
20\end{array}$ & $\begin{array}{l}29387.04 \\
29384.37 \\
29368.07 \\
29350.79 \\
29346.47\end{array}$ & $\begin{array}{l}+0.04 \\
+0.11 \\
+0.07 \\
+0.09 \\
+0.15\end{array}$ & $\begin{array}{c}a^{4} \mathrm{D}_{21 / 2}-44354^{\circ}{ }^{\circ 1 / 2} \\
24991_{11 / 2}-54375_{21 / 2}^{\circ} \\
29341_{41 / 2}-58709^{\circ}{ }^{\circ} 1 / 2 \\
25672_{21 / 2}-55022_{31 / 2}^{\circ} \\
29341_{41 / 2}-58687_{41 / 2}^{\circ}\end{array}$ & res \\
\hline $\begin{array}{l}\text { 3406. } 824 \\
3407.450 \\
3408.214 \\
3410.170 \\
3412.736\end{array}$ & $\begin{array}{c}30 A \\
2 \\
2 \\
1 \\
3\end{array}$ & $\begin{array}{r}40 \\
10 \\
5 \\
25 \\
30\end{array}$ & $\begin{array}{l}29344.43 \\
29339.04 \\
29332.47 \\
29315.64 \\
29293.60\end{array}$ & $\begin{array}{l}+0.14 \\
+0.16 \\
+0.19 \\
-0.09 \\
+0.08\end{array}$ & $\begin{array}{r}a^{4} \mathrm{P}_{11 / 2}-39936_{21 / 2}^{\circ} \\
27273_{31 / 2}-56612_{31 / 2}^{\circ}-52567^{\circ 1 / 2} \\
23234_{41 / 2}-5245_{01 / 2}^{\circ} \\
25169_{11 / 2}-544894^{\circ} 1 / 2 \\
18990_{11 / 2}-482\end{array}$ & res \\
\hline $\begin{array}{l}3415.406 \\
3416.370 \\
3416.623 \\
3417.620 \\
3419.720\end{array}$ & $\begin{array}{r}4 \\
20\end{array}$ & $\begin{array}{r}6 \\
2 \\
100 \\
3 \\
5\end{array}$ & $\begin{array}{l}29270.70 \\
29262.44 \\
29260.27 \\
29251.74 \\
29233.77\end{array}$ & $\begin{array}{l}+0.16 \\
+0.39 \\
-0.13 \\
+0.14\end{array}$ & $\begin{array}{l}27273_{31 / 2}-56544_{21 / 2}^{\circ} \\
19070_{41 / 2}-48332_{51 / 2}^{\circ} \\
24804_{31 / 2}-54056_{41 / 2}^{\circ} \\
26158_{41 / 2}^{\circ}-55392_{41 / 2}^{\circ}\end{array}$ & \\
\hline $\begin{array}{l}3421.134 \\
3421.430 \\
3422.742 \\
3424.436 \\
3426.20\end{array}$ & $\begin{array}{l}4 \\
1 \\
5\end{array}$ & $\begin{array}{r}40 \\
5 \\
6 \\
50 \\
4\end{array}$ & $\begin{array}{l}29221.69 \\
29219.17 \\
29207.97 \\
29193.52 \\
29178.5\end{array}$ & $\begin{array}{l}+0.12 \\
+0.07 \\
+0.17 \\
+0.20 \\
+0.3\end{array}$ & $\begin{array}{r}24804_{31 / 2}-54026_{21 / 2}^{\circ}-54137^{\circ 1 / 2} \\
24918_{11 / 2}-541354_{21 / 2}^{\circ} \\
a^{4} \mathrm{D}_{31 / 2}-44380_{31 / 2}^{\circ} \\
19637_{21 / 2}-48898_{41 / 2}^{\circ} \\
31100_{51 / 2}-6027\end{array}$ & res \\
\hline $\begin{array}{l}3430.558 \\
3430.739 \\
3435.636 \\
3435.710 \\
3437.233\end{array}$ & $\begin{array}{c}1- \\
30 A \\
3\end{array}$ & $\begin{array}{c}1 \\
8 \\
4 \\
6 \\
20 ?\end{array}$ & $\begin{array}{l}29141.42 \\
29139.89 \\
29098.35 \\
29097.73 \\
29084.83\end{array}$ & $\begin{array}{l}+0.12 \\
+0.11 \\
+0.29 \\
-0.17 \\
+0.05\end{array}$ & $\begin{array}{l}20039_{31 / 2}-49181_{11 / 2}^{\circ} \\
27273_{31 / 2}-56413_{41 / 2}^{\circ} \\
28631_{31 / 2}-57799_{31 / 2}^{\circ} \\
23803_{31 / 2}-52901_{31 / 2}^{\circ} \\
20039_{31 / 2}-49124_{31 / 2}^{\circ}\end{array}$ & $\begin{array}{l}d \\
\text { res }\end{array}$ \\
\hline $\begin{array}{l}3440.583 \\
3440.644 \\
3442.493 \\
3443.167 \\
3444.934\end{array}$ & $\begin{array}{l}4 \\
2\end{array}$ & $\begin{array}{r}20 \\
200 \\
20 \\
2 \\
2\end{array}$ & $\begin{array}{l}29056.51 \\
29056.00 \\
29040.40 \\
29034.71 \\
29019.82\end{array}$ & $\begin{array}{l}+0.10 \\
+0.06 \\
+0.18 \\
+0.03 \\
+0.04\end{array}$ & $\begin{array}{c}a^{4} \mathrm{G}_{51 / 2}-z^{6} \mathrm{~F}_{41 / 2}^{\circ} \\
33910_{51 / 2}-62966_{51 / 2}^{\circ} \\
23046_{31 / 2}-52087^{\circ}{ }_{21 / 2}^{\circ} \\
24991_{11 / 2}-54026_{21 / 2}^{\circ} \\
25209_{41 / 2}-54229_{51 / 2}^{\circ}\end{array}$ & res \\
\hline $\begin{array}{l}3449.862 \\
3451.166 \\
3452.494 \\
3454.962 \\
3455.015\end{array}$ & $\begin{array}{l}10 \\
8 \\
1 d\end{array}$ & $\begin{array}{l}100 \\
10 \\
30 \\
30 d\end{array}$ & $\begin{array}{l}28978.36 \\
28967.41 \\
28956.27 \\
28935.54 \\
28935.14\end{array}$ & $\begin{array}{l}+0.05 \\
+0.08 \\
+0.10 \\
+0.24 \\
+0.17\end{array}$ & 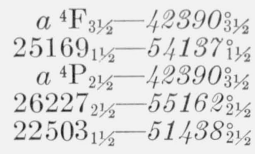 & $\begin{array}{l}6 \\
7 \\
\text { res } \\
5\end{array}$ \\
\hline
\end{tabular}


Table 3. Classified lines of $\mathrm{W} \mathrm{II}$-Continued

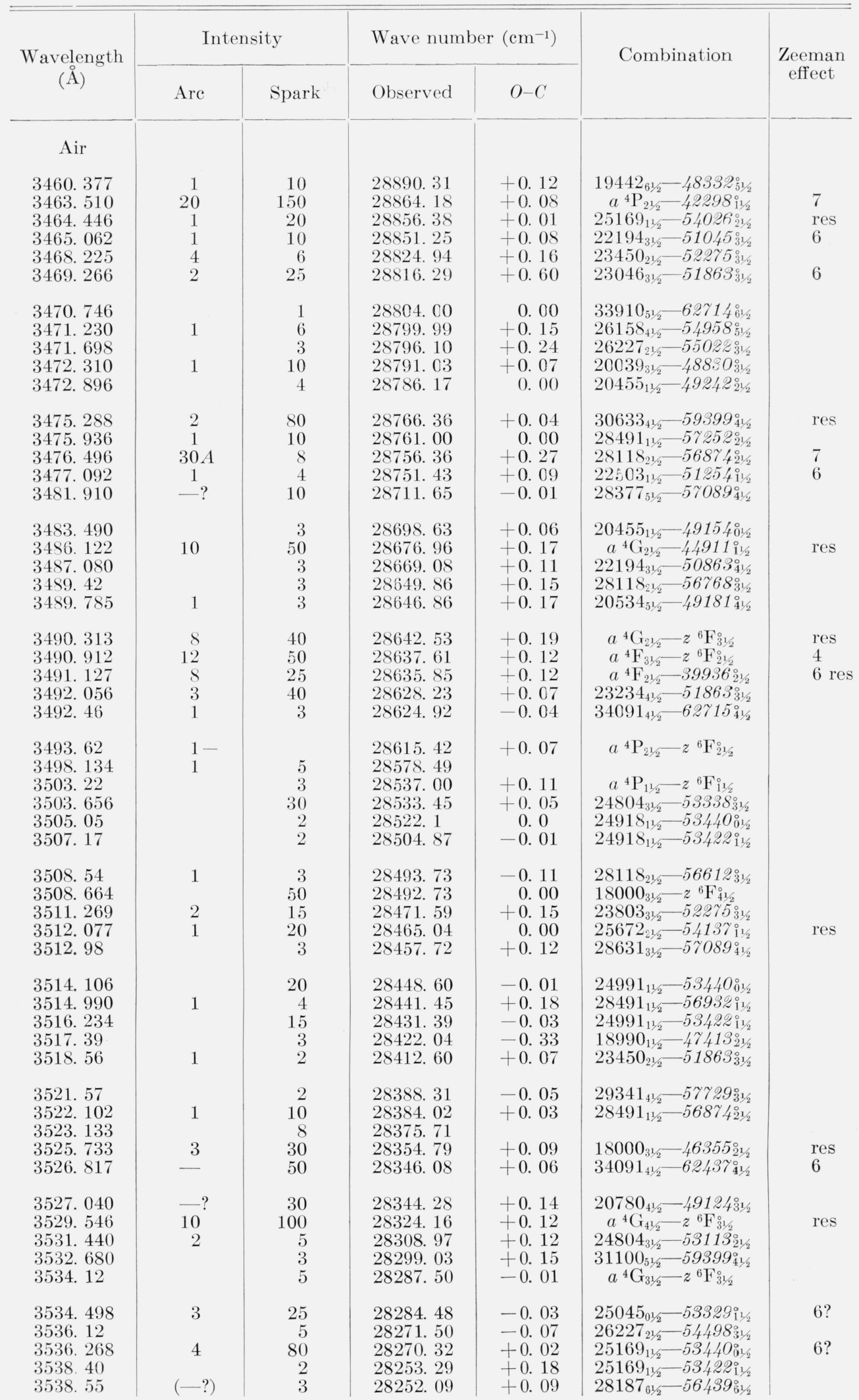


TABLE 3. Classified lines of $\mathrm{W} \mathrm{II}$-Continued

\begin{tabular}{|c|c|c|c|c|c|c|}
\hline \multirow{2}{*}{$\begin{array}{l}\text { Wavelength } \\
(\AA)\end{array}$} & \multicolumn{2}{|c|}{ Intensity } & \multicolumn{2}{|c|}{ Wave number $\left(\mathrm{cm}^{-1}\right)$} & \multirow{2}{*}{ Combination } & \multirow{2}{*}{$\begin{array}{c}\text { Zeeman } \\
\text { effect }\end{array}$} \\
\hline & Are & Spark & Observed & $O-C$ & & \\
\hline \multicolumn{7}{|l|}{ Air } \\
\hline $\begin{array}{l}3539.455 \\
3544.460 \\
3546.474 \\
3547.00 \\
3548.258\end{array}$ & $\begin{array}{l}3 A \\
5 \\
3 A \\
1 \\
15 A\end{array}$ & $\begin{array}{r}4 \\
25 \\
3 \\
\\
12\end{array}$ & $\begin{array}{l}28244.87 \\
28204.98 \\
28188.97 \\
28184.79 \\
28174.79\end{array}$ & $\begin{array}{l}+0.13 \\
+0.02 \\
-0.01 \\
+0.23 \\
+0.07\end{array}$ & $\begin{array}{r}20039_{31 / 2}-48284^{\circ} 1 \frac{1}{2} \\
a^{4} \mathrm{G}_{41 / 2}-44758^{\circ} 1 / 2 \\
18990_{11 / 2}-47179_{11 / 2}^{\circ} \\
19404_{01 / 2}-47588_{11 / 2}^{\circ} \\
18000_{31 / 2}-46175_{31 / 2}^{\circ}\end{array}$ & $\begin{array}{l}\text { res } \\
\text { res } \\
d\end{array}$ \\
\hline $\begin{array}{l}3549.052 \\
3550.14 \\
3551.528 \\
3553.020 \\
3554.068\end{array}$ & 25 & $\begin{array}{r}150 \\
3 \\
10 \\
5 \\
3\end{array}$ & $\begin{array}{l}28168.49 \\
28159.86 \\
28148.85 \\
28137.03 \\
28128.74\end{array}$ & $\begin{array}{l}+0.06 \\
+0.02 \\
+0.03 \\
+0.22 \\
-0.05\end{array}$ & $\begin{array}{c}a^{4} \mathrm{G}_{31 / 2}-44758_{41 / 2}^{\circ}-53329_{11 / 2}^{\circ} \\
25169_{11 / 2}-57^{\circ} \\
26227_{21 / 2}-54375_{21 / 2}^{\circ} \\
19276_{21 / 2}-47413_{21 / 2}^{\circ} \\
25209_{41 / 2}-53338_{31 / 2}^{\circ}\end{array}$ & res \\
\hline $\begin{array}{l}3554.93 \\
3555.166 \\
3557.914 \\
3560.67 \\
3561.456\end{array}$ & $\begin{array}{r}12 \\
5\end{array}$ & $\begin{array}{r}2 \\
120 \\
30 \\
2 \\
5\end{array}$ & $\begin{array}{l}28121.92 \\
28120.05 \\
28098.33 \\
28076.58 \\
28070.39\end{array}$ & $\begin{array}{l}-0.04 \\
+0.07 \\
+0.08 \\
+0.04 \\
+0.07\end{array}$ & $\begin{array}{r}24991_{11 / 2}-53113_{21 / 2}^{\circ} \\
a^{4} \mathrm{G}_{21 / 2}-44354_{21 / 2}^{\circ} \\
22194_{31 / 2}-50292_{21 / 2}^{\circ} \\
30633_{41 / 2}-58709^{\circ}{ }_{1 / 2} \\
26158_{41 / 2}-54299^{\circ}{ }^{1 / 2}\end{array}$ & res \\
\hline $\begin{array}{l}3562.508 \\
3563.59 \\
3563.998 \\
3565.834 \\
3566.73\end{array}$ & $\begin{array}{l}1 \\
1 \\
1\end{array}$ & $\begin{array}{l}5 \\
3 \\
2 \\
3 \\
3\end{array}$ & $\begin{array}{l}28062.10 \\
28053.6 \\
28050.37 \\
28035.92 \\
28028.9\end{array}$ & $\begin{array}{l}+0.30 \\
+0.2 \\
+0.05 \\
+0.08 \\
-0.3\end{array}$ & $\begin{array}{l}28377_{51 / 2}-56439_{61 / 2}^{\circ} \\
28491_{11 / 2}-565442^{11 / 2} \\
20780_{41 / 2}-48830_{31 / 2}^{\circ} \\
28377_{51 / 2}-56413_{41 / 2}^{\circ} \\
26929_{51 / 2}-54958_{51 / 2}^{\circ}\end{array}$ & 4 \\
\hline $\begin{array}{l}3571.972 \\
3572.474 \\
3572.830 \\
3574.82 \\
3577.59\end{array}$ & $\begin{array}{r}3 \\
60 \\
1\end{array}$ & $\begin{array}{r}8 \\
200 \\
5 \\
2 \\
2\end{array}$ & $\begin{array}{l}27987.75 \\
27983.82 \\
27981.03 \\
27965.4 \\
27943.8\end{array}$ & $\begin{array}{l}+0.22 \\
+0.02 \\
+0.15 \\
0.0 \\
+0.2\end{array}$ & $\begin{array}{c}23450_{21 / 2}-51438_{21 / 2}^{\circ} \\
a^{4} \mathrm{P}_{11 / 2}-z^{2} \mathrm{~S}_{01 / 2}^{\circ} \\
28631_{31 / 2}-56612^{\circ}{ }^{11 / 2} \\
28118_{21 / 2}-56084_{11 / 2}^{\circ} \\
25169_{11 / 2}-53113_{21 / 2}^{\circ}\end{array}$ & $\begin{array}{l}\text { res } \\
6\end{array}$ \\
\hline $\begin{array}{l}3579.64 \\
3581.876 \\
3582.75 \\
3583.458 \\
3584.68\end{array}$ & $\begin{array}{l}2 \\
1 \\
1\end{array}$ & $\begin{array}{l}3 \\
6 \\
2 \\
8 \\
3\end{array}$ & $\begin{array}{l}27927.8 \\
27910.36 \\
27903.56 \\
27898.04 \\
27888.53\end{array}$ & $\begin{array}{l}-0.1 \\
+0.16 \\
+0.14 \\
+0.24 \\
+0.09\end{array}$ & $\begin{array}{l}22503_{11 / 2}-50430_{11 / 2}^{\circ} \\
26227_{21 / 2}-54137_{11 / 2}^{\circ} \\
19276_{21 / 2}-47179_{11 / 2}^{\circ} \\
26158_{41 / 2}-54056_{41 / 2}^{\circ} \\
27273_{31 / 2}-55162_{21 / 2}^{\circ}\end{array}$ & $\begin{array}{l}\text { res } \\
6\end{array}$ \\
\hline 3592. 418 & 50 & 150 & 27828. 46 & $\left\{\begin{array}{l}-0.09 \\
+0.13\end{array}\right.$ & $\begin{array}{c}20455_{11 / 2}-48284{ }^{\circ 1 / 2} \\
a^{4} \mathrm{~F}_{21 / 2}-z^{6} \mathrm{~F}_{11 / 2}^{0}\end{array}$ & $\begin{array}{l}5 \text { res } \\
4\end{array}$ \\
\hline $\begin{array}{l}3596.171 \\
3597.48 \\
3598.45 \\
3600.594\end{array}$ & $\frac{2}{2} ?$ & $\begin{array}{r}30 \\
2 \\
20 \\
1\end{array}$ & $\begin{array}{l}27799.42 \\
27789.31 \\
27781.8 \\
27765.27\end{array}$ & $\begin{array}{r}+0.18 \\
+0.04 \\
0.0 \\
+0.12\end{array}$ & $\begin{array}{r}26227_{21 / 2}-54026^{\circ}{ }_{21 / 2} \\
22503_{11 / 2}-50292_{21 / 2}^{\circ} \\
28631_{31 / 2}-56413^{\circ} 11 / 2 \\
a^{4} \mathrm{G}_{31 / 2}-4435421 / 2\end{array}$ & 7 \\
\hline $\begin{array}{l}3600.933 \\
3601.55 \\
3602.455 \\
3602.700 \\
3608.280\end{array}$ & $\begin{array}{l}1 \\
3 \\
-?\end{array}$ & $\begin{array}{r}6 \\
1 \\
8 \\
1 \\
10\end{array}$ & $\begin{array}{l}27762.66 \\
27757.90 \\
27750.93 \\
27749.04 \\
27706.13\end{array}$ & $\begin{array}{l}+0.18 \\
+0.10 \\
+0.11 \\
+0.04\end{array}$ & $\begin{array}{l}24804_{31 / 2}-52567_{41 / 2}^{\circ} \\
25045_{01 / 2}-52803_{11 / 2}^{11} \\
25672_{21 / 2}-53422_{11 / 2}^{\circ} \\
27273_{31 / 2}-55022_{31 / 2}^{\circ}\end{array}$ & \\
\hline $\begin{array}{l}3610.044 \\
3611.826 \\
3613.530 \\
3613.785 \\
3614.606\end{array}$ & $\begin{array}{r}1 \\
1 \\
30 \\
1\end{array}$ & $\begin{array}{r}20 \\
20 \\
4 \\
150 \\
8\end{array}$ & $\begin{array}{l}27692.59 \\
27678.92 \\
27665.88 \\
27663.93 \\
27657.64\end{array}$ & $\begin{array}{r}+0.13 \\
+0.02 \\
-0.03 \\
+0.09 \\
+0.09\end{array}$ & $\begin{array}{r}25209_{41 / 2}-52901_{31 / 2}^{\circ} \\
33910_{51 / 2}-61589^{\circ} 1 / 2 \\
25672_{21 / 2}-53938_{31 / 2}^{\circ} \\
a^{4} \mathrm{D}_{11 / 2}^{\circ}-42298_{11 / 2}^{\circ} \\
25672_{21 / 2}-53329_{11 / 2}^{\circ}\end{array}$ & $\begin{array}{l}\text { res } \\
6 \\
\text { res }\end{array}$ \\
\hline 3617.652 & & 4 & 27634. 36 & $\left\{\begin{array}{l}+0.05 \\
+0.17\end{array}\right.$ & $\begin{array}{l}18990_{11 / 2}-46625_{01 / 2}^{\circ} \\
23803_{31 / 2}-51438_{21 / 2}^{\circ}\end{array}$ & \\
\hline $\begin{array}{l}3618.434 \\
3628.413 \\
3628.924 \\
3630.080\end{array}$ & $\begin{array}{l}1 \\
3 \\
2 \\
2\end{array}$ & $\begin{array}{r}25 \\
30 \\
25 \\
3\end{array}$ & $\begin{array}{l}27628.39 \\
27552.40 \\
27548.52 \\
27539.75\end{array}$ & $\begin{array}{r}+0.21 \\
+0.05 \\
0.00 \\
0.15\end{array}$ & $\begin{array}{l}23234_{41 / 2}-50863_{41 / 2}^{\circ} \\
20780_{41 / 2}-48332^{\circ}{ }^{11 / 2} \\
25045_{01 / 2}-52593_{01 / 2}^{\circ} \\
23955_{51 / 2}-z^{6} \mathrm{~F}_{51 / 2}^{\circ}\end{array}$ & $\begin{array}{l}\text { res } \\
\text { res }\end{array}$ \\
\hline $\begin{array}{l}3630.950 \\
3639.213 \\
3641.403 \\
3645.594 \\
3646.577\end{array}$ & $\begin{array}{l}60 \\
10 \\
10\end{array}$ & $\begin{array}{r}8 \\
1 \\
120 \\
100 \\
50\end{array}$ & $\begin{array}{l}27533.15 \\
27470.64 \\
27454.12 \\
27422.56 \\
27415.16\end{array}$ & $\begin{array}{l}+0.10 \\
+0.03 \\
+0.03 \\
+0.11 \\
+0.07\end{array}$ & $\begin{array}{c}a^{4} \mathrm{~F}_{41 / 2}-42390_{31 / 2}^{\circ} \\
24804_{31 / 2}^{\circ}-52275_{31 / 2}^{\circ} \\
a^{4} \mathrm{~F}_{11 / 2}-z^{6} \mathrm{~F}_{01 / 2}^{\circ} \\
a^{4} \mathrm{D}_{21 / 2}-42390^{\circ}{ }^{\circ 1 / 2} \\
a^{4} \mathrm{D}_{11 / 2}-z^{6} \mathrm{~F}_{21 / 2}^{\circ}\end{array}$ & $\begin{array}{l}7 \\
\text { res }\end{array}$ \\
\hline
\end{tabular}


Table 3. Classified lines of $\mathrm{W} \mathrm{II}$-Continued

\begin{tabular}{|c|c|c|c|c|c|c|}
\hline \multirow{2}{*}{$\begin{array}{c}\text { Wavelength } \\
(\AA)\end{array}$} & \multicolumn{2}{|c|}{ Intensity } & \multicolumn{2}{|c|}{ Wave number $\left(\mathrm{cm}^{-1}\right)$} & \multirow{2}{*}{ Combination } & \multirow{2}{*}{$\begin{array}{c}\text { Zeeman } \\
\text { effect }\end{array}$} \\
\hline & Arc & Spark & Observed & $O-C$ & & \\
\hline \multicolumn{7}{|l|}{ Air } \\
\hline $\begin{array}{l}3652.114 \\
3653.324 \\
3654.74 \\
3657.583 \\
3657.871\end{array}$ & $\begin{array}{r}10 \\
2 \\
\\
40 \\
10\end{array}$ & $\begin{array}{r}20 \\
10 \\
10 \\
120 \\
40\end{array}$ & $\begin{array}{l}27373.60 \\
27364.54 \\
27353.94 \\
27332.67 \\
27330.52\end{array}$ & $\begin{array}{l}+0.01 \\
+0.10 \\
+0.04 \\
+0.02 \\
+0.14\end{array}$ & $\begin{array}{c}20039_{31 / 2}-47413_{21 / 2}^{\circ} \\
18990_{11 / 2}-46355^{\circ 1 / 2} \\
30633^{41 / 2}-57986^{\circ}{ }^{\circ} 1 / 2 \\
a^{4} \mathrm{P}_{01 / 2}-z^{6} \mathrm{~F}_{01 / 2}^{\circ} \\
a^{4} \mathrm{D}_{21 / 2}-42298_{11 / 2}^{\circ}\end{array}$ & res \\
\hline $\begin{array}{l}3660.604 \\
3670.65 \\
3672.547 \\
3677.405 \\
3678.210\end{array}$ & $\begin{array}{l}30 A \\
5 \\
1 \\
1\end{array}$ & $\begin{array}{c}8 \\
1 ? \\
25 \\
8 \\
10\end{array}$ & $\begin{array}{l}27310.12 \\
27235.37 \\
27221.31 \\
27185.35 \\
27179.40\end{array}$ & $\begin{array}{l}+0.21 \\
+0.10 \\
+0.12 \\
+0.11 \\
+0.07\end{array}$ & $\begin{array}{l}25045_{01 / 2}-52355_{01 / 2}^{\circ} \\
34091_{41 / 2}-61326_{31 / 2}^{\circ} \\
19404_{01 / 2}-46625_{01 / 2}^{\circ} \\
25169_{11 / 2}-52355_{01 / 2}^{\circ} \\
26158_{41 / 2}-53338_{31 / 2}^{\circ}\end{array}$ & $\begin{array}{l}6 \\
\text { res }\end{array}$ \\
\hline $\begin{array}{l}3687.510 \\
3689.596 \\
3691.463 \\
3691.846 \\
3692.864\end{array}$ & $\begin{array}{l}1 \\
4\end{array}$ & $\begin{array}{r}2 \\
10 \\
2 \\
40 \\
2\end{array}$ & $\begin{array}{l}27110.85 \\
27095.52 \\
27081.82 \\
27079.01 \\
27071.55\end{array}$ & $\begin{array}{l}-0.22 \\
+0.06 \\
+0.19 \\
+0.13 \\
-0.53\end{array}$ & $\begin{array}{c}26227_{21 / 2}-53338_{31 / 2}^{\circ} \\
24991_{11 / 2}^{\circ}-52087_{21 / 2}^{\circ} \\
a^{4} \mathrm{D}_{21 / 2}-z^{6} \mathrm{~F}_{21 / 2}^{\circ} \\
19276_{21 / 2}^{\circ}-46355_{21 / 2}^{\circ} \\
29341_{41 / 2}^{\circ}-56413_{41 / 2}^{\circ}\end{array}$ & res \\
\hline $\begin{array}{l}3694.525 \\
3705.310 \\
3707.478 \\
3708.494 \\
3712.202\end{array}$ & $\begin{array}{c}40 A \\
1 \\
3 \\
8 \\
3\end{array}$ & $\begin{array}{r}40 \\
4 \\
2 \\
60 \\
12\end{array}$ & $\begin{array}{l}27059.38 \\
26980.62 \\
26964.84 \\
26957.45 \\
26930.53\end{array}$ & $\begin{array}{l}+0.17 \\
+0.17 \\
+0.06 \\
+0.05 \\
+0.09\end{array}$ & $\begin{array}{l}23803_{31 / 2}-50863_{41 / 2}^{\circ} \\
23450_{21 / 2}^{\circ}-50430_{11 / 2}^{\circ} \\
34091_{41 / 2}^{\circ}-61055_{41 / 2}^{\circ} \\
20455_{11 / 2}-47413_{21 / 2}^{\circ} \\
22194_{31 / 2}-49124_{31 / 2}^{\circ}\end{array}$ & 5 \\
\hline $\begin{array}{l}3714.033 \\
3715.832 \\
3716.062 \\
3716.550 \\
3719.66\end{array}$ & $\begin{array}{r}3 \\
10 \\
2\end{array}$ & $\begin{array}{r}2 \\
8 \\
50 \\
2 \\
3\end{array}$ & $\begin{array}{l}26917.25 \\
26904.22 \\
26902.55 \\
26899.02 \\
26876.53\end{array}$ & $\begin{array}{l}+0.10 \\
+0.26 \\
+0.12 \\
+0.12 \\
+0.05\end{array}$ & $\begin{array}{c}25169_{11 / 2}-52087^{\circ 1 / 2} \\
28118_{21 / 2}-55022^{\circ}{ }^{11 / 2} \\
a^{4} \mathrm{D}_{31 / 2}-z^{6} \mathrm{~F}_{21 / 2}^{\circ} \\
19276_{21 / 2}-46175^{\circ}{ }^{\circ 1 / 2} \\
18000_{31 / 2}-z^{6} \mathrm{~F}_{31 / 2}^{\circ}\end{array}$ & res \\
\hline $\begin{array}{l}3721.102 \\
3732.684 \\
3736.213 \\
3736.91 \\
3741.706\end{array}$ & $\begin{array}{l}2 \\
12 \\
30 A\end{array}$ & $\begin{array}{r}10 \\
2 \\
60 \\
2 \\
12\end{array}$ & $\begin{array}{l}26866.12 \\
26782.76 \\
26757.46 \\
26752.5 \\
26718.18\end{array}$ & $\begin{array}{l}+0.08 \\
+0.06 \\
+0.1 \\
+0.16\end{array}$ & $\begin{array}{l}27273_{31 / 2}-54056_{41 / 2}^{\circ} \\
18000_{31 / 2}-44758_{41 / 2}^{\circ} \\
27273_{31 / 2}-54026_{21 / 2}^{\circ} \\
19637_{21 / 2}^{\circ}-46355_{21 / 2}^{\circ}\end{array}$ & $\begin{array}{l}\text { res } \\
6\end{array}$ \\
\hline $\begin{array}{l}3745.530 \\
3750.744 \\
3753.162 \\
3753.620 \\
3760.364\end{array}$ & $\begin{array}{l}4 \\
1\end{array}$ & $\begin{array}{r}40 \\
8 \\
3 \\
10 \\
20\end{array}$ & $\begin{array}{l}26690.90 \\
26653.80 \\
26636.63 \\
26633.38 \\
26585.61\end{array}$ & $\begin{array}{l}+0.17 \\
+0.05 \\
+0.01 \\
+0.02 \\
-0.10\end{array}$ & $\begin{array}{l}22139_{21 / 2}-48830_{31 / 2}^{\circ} \\
25209_{41 / 2}-51863_{31 / 2}^{\circ} \\
22194_{31 / 2}-48830_{31 / 2}^{\circ} \\
24804_{31 / 2}-51438_{21 / 2}^{\circ} \\
28118_{21 / 2}-54704_{21 / 2}^{\circ}\end{array}$ & 7 \\
\hline $\begin{array}{l}3763.616 \\
3768.97 \\
3772.05 \\
3774.14 \\
3777.35\end{array}$ & $\begin{array}{l}2 \\
\frac{8 A}{1}\end{array}$ & $\begin{array}{r}2 \\
4 \\
3 \\
20 \\
10\end{array}$ & $\begin{array}{l}26562.64 \\
26524.91 \\
26503.25 \\
26488.58 \\
26466.07\end{array}$ & $\begin{array}{l}-0.10 \\
+0.06 \\
+0.54 \\
+0.09 \\
+0.01\end{array}$ & $\begin{array}{r}18990_{11 / 2}-45553_{11 / 2}^{\circ} \\
a^{4} \mathrm{~F}_{31 / 2}-39936_{21 / 2}^{\circ} \\
a^{4} \mathrm{P}_{21 / 2}-39936_{21 / 2}^{\circ} \\
23803_{31 / 2}^{\circ}-50290_{21 / 2}^{\circ} \\
18990_{11 / 2}-45457_{01 / 2}^{\circ}\end{array}$ & \\
\hline $\begin{array}{l}3778.686 \\
3785.58 \\
3789.726 \\
3804.481 \\
3814.418\end{array}$ & $30 A$ & $\begin{array}{r}8 \\
20 \\
20 \\
2 \\
6\end{array}$ & $\begin{array}{l}26456.71 \\
26408.5 \\
26379.64 \\
26277.3 \\
26208.88\end{array}$ & $\begin{array}{l}+0.27 \\
+0.1 \\
-0.03 \\
+0.1 \\
-0.32\end{array}$ & $\begin{array}{l}30633_{41 / 2}-57089_{41 / 2}^{\circ} \\
26158_{41 / 2}-52567_{41 / 2}^{\circ} \\
28118_{21 / 2}-54498_{31 / 2}^{\circ} \\
19276_{21 / 2}-45553_{11 / 2}^{\circ} \\
25045_{11 / 2}-51254_{11 / 2}^{\circ}\end{array}$ & \\
\hline $\begin{array}{l}3822.198 \\
3823.062 \\
3827.89 \\
3833.61 \\
3837.238\end{array}$ & $\begin{array}{l}2 \\
3 \\
2 \\
4\end{array}$ & $\begin{array}{r}4 \\
10 \\
4 \\
\\
10\end{array}$ & $\begin{array}{l}26155.53 \\
26149.62 \\
26116.6 \\
26077.7 \\
26053.02\end{array}$ & $\begin{array}{r}+0.10 \\
0.00 \\
+0.1 \\
0.0 \\
+0.08\end{array}$ & $\begin{array}{r}a^{4} \mathrm{G}_{21 / 2}-42390_{31 / 2}^{\circ} \\
19404_{01 / 2}-45553_{11 / 2}^{\circ} \\
26158_{41 / 2}-52275_{31 / 2}^{\circ} \\
23046_{31 / 2}^{\circ}-49124^{\circ}{ }^{31 / 2} \\
19404_{01 / 2}-45457_{01 / 2}^{\circ}\end{array}$ & res \\
\hline $\begin{array}{l}3846.688 \\
3851.131 \\
3851.570 \\
3856.828 \\
3859.986\end{array}$ & $\begin{array}{c}2 \\
\frac{2}{12} \\
3 \\
2\end{array}$ & $\begin{array}{r}2 \\
60 \\
6 \\
6\end{array}$ & $\begin{array}{l}25989.02 \\
25959.03 \\
25956.07 \\
25920.69 \\
25899.48\end{array}$ & $\begin{array}{l}+0.02 \\
-0.05 \\
+0.04 \\
+0.02 \\
+0.01\end{array}$ & $\begin{array}{c}31100_{51 / 2}-57089^{\circ}{ }_{41 / 2} \\
20534_{51 / 2}-z^{6} \mathrm{~F}_{41 / 2}^{\circ} \\
a^{4} \mathrm{D}_{01 / 2}-z^{6} \mathrm{~F}_{11 / 2}^{\circ} \\
18990_{11 / 2}-44911_{11 / 2}^{\circ} \\
20455_{11 / 2}-46355_{21 / 2}^{\circ}\end{array}$ & $\begin{array}{l}\text { res } \\
6\end{array}$ \\
\hline
\end{tabular}


Table 3. Classified lines of W II-Continued

\begin{tabular}{|c|c|c|c|c|c|c|}
\hline \multirow{2}{*}{$\begin{array}{l}\text { Wavelength } \\
(\AA)\end{array}$} & \multicolumn{2}{|c|}{ Intensity } & \multicolumn{2}{|c|}{ Wave number $\left(\mathrm{cm}^{-1}\right)$} & \multirow{2}{*}{ Combination } & \multirow{2}{*}{$\begin{array}{c}\text { Zeeman } \\
\text { effect }\end{array}$} \\
\hline & Arc & Spark & Observed & $O-C$ & & \\
\hline \multicolumn{7}{|l|}{ Air } \\
\hline $\begin{array}{l}3872.656 \\
3877.29 \\
3877.65 \\
3883.336 \\
3887.951\end{array}$ & $\begin{array}{l}4 \\
2 \\
1 \\
2 \\
1\end{array}$ & $\begin{array}{r}10 \\
15 \\
\\
3 \\
3\end{array}$ & $\begin{array}{l}25814.75 \\
25783.9 \\
25781.5 \\
25743.76 \\
25713.20\end{array}$ & $\begin{array}{l}+0.14 \\
0.0 \\
+0.1 \\
-0.20 \\
+0.15\end{array}$ & $\begin{array}{c}a^{4} \mathrm{G}_{21 / 2}-z^{6} \mathrm{~F}_{21 / 2}^{\circ} \\
23046_{31 / 2}-48830_{31 / 2}^{\circ} \\
22503_{11 / 2}-48284^{\circ}{ }^{21 / 2} \\
28631_{31 / 2}-54375_{21 / 2}^{\circ} \\
20780_{41 / 2}-z^{6} \mathrm{~F}_{41 / 2}^{\circ}\end{array}$ & 6 \\
\hline $\begin{array}{l}3890.658 \\
3891.83 \\
3896.930 \\
3899.782 \\
3900.879\end{array}$ & $\begin{array}{l}-? \\
1 \\
3 \\
2 \\
2\end{array}$ & $\begin{array}{r}2 \\
1 \\
20 \\
3 \\
8\end{array}$ & $\begin{array}{l}\text { 25695. } 31 \\
25687.57 \\
25653.95 \\
25635.19 \\
25627.98\end{array}$ & $\begin{array}{r}0.00 \\
+0.15 \\
+0.18 \\
+0.08 \\
+0.10\end{array}$ & $\begin{array}{c}a^{4} \mathrm{P}_{21 / 2}-z{ }^{6} \mathrm{~F}_{11 / 2}^{\circ} \\
19070_{41 / 2}-44758_{41 / 2}^{\circ} \\
25209_{41 / 2}-50863_{41 / 2}^{\circ} \\
19276_{21 / 2}-44911_{11 / 2}^{\circ} \\
27273_{31 / 2}-52901_{31 / 2}^{\circ}\end{array}$ & 6 \\
\hline $\begin{array}{l}\text { 3905. } 79 \\
3909.276 \\
3915.456 \\
3935.43 \\
3936.653\end{array}$ & $\begin{array}{l}1 \\
2 \\
2 \\
4 \\
1\end{array}$ & $\begin{array}{r}3 \\
2 \\
5 \\
10 \\
5\end{array}$ & $\begin{array}{l}\text { 25595. } 8 \\
25572.94 \\
25532.57 \\
25403.0 \\
25395.10\end{array}$ & $\begin{array}{l}0.0 \\
+0.11 \\
+0.21 \\
+0.1 \\
+0.06\end{array}$ & $\begin{array}{c}23234_{41 / 2}-48830_{31 / 2}^{\circ} \\
a^{4} \mathrm{P}_{11 / 2}-z^{6} \mathrm{~F}_{01 / 2}^{\circ} \\
23450_{21 / 2}-48982_{11 / 2}^{\circ} \\
a^{4} \mathrm{D}_{01 / 2}-z^{2} \mathrm{~S}_{01 / 2}^{\circ} \\
20780_{41 / 2}-46175_{31 / 2}^{\circ}\end{array}$ & \\
\hline $\begin{array}{l}\text { 3936. } 782 \\
3938.10 \\
3939.43 \\
3951.067 \\
3955.606\end{array}$ & $\begin{array}{l}1 \\
8 \\
3 \\
3 \\
1\end{array}$ & $\begin{array}{l}1 \\
3 \\
6 \\
1\end{array}$ & $\begin{array}{l}\text { 25394. } 27 \\
25385.8 \\
25377.2 \\
25302.45 \\
25273.42\end{array}$ & $\begin{array}{r}-0.11 \\
0.0 \\
0.0 \\
0.00 \\
+0.06\end{array}$ & $\begin{array}{r}28631_{31 / 2}-54026_{21 / 2}^{\circ} \\
25045_{01 / 2}-50430_{11 / 2}^{\circ} \\
23803_{31 / 2}-49181_{41 / 2}^{\circ} \\
a^{4} \mathrm{D}_{11 / 2}-39936_{21 / 2}^{\circ} \\
22139_{21 / 2}^{\circ}-47413_{21 / 2}^{\circ}\end{array}$ & \\
\hline $\begin{array}{l}\text { 3960. } 87 \\
3691.192 \\
3964.096 \\
3983.292 \\
3986.38\end{array}$ & $\begin{array}{l}2 \\
-? \\
-? \\
50 \mathrm{~A}\end{array}$ & $\begin{array}{l}6 \\
3 \\
6 \\
5 \\
1\end{array}$ & $\begin{array}{l}\text { 25239. } 83 \\
25237.78 \\
25219.29 \\
25097.76 \\
25078.3\end{array}$ & $\begin{array}{l}+0.03 \\
+0.10 \\
+0.04 \\
-0.01 \\
\quad 0.0\end{array}$ & $\begin{array}{l}19637_{21 / 2}-z^{6} \mathrm{~F}_{31 / 2}^{\circ} \\
23046_{31 / 2}-48284^{\circ}{ }^{11 / 2} \\
22194_{31 / 2}-47413_{21 / 2}^{\circ} \\
20455_{11 / 2}-45553_{11 / 2}^{\circ} \\
19276_{21 / 2}-4435421 \frac{1}{2}\end{array}$ & $6, d$ \\
\hline $\begin{array}{l}3990.42 \\
3994.56 \\
4000.10 \\
4001.883 \\
4060.166\end{array}$ & $\begin{array}{l}2 \\
1 \\
1 \\
1\end{array}$ & $\begin{array}{l}2 \\
1 \\
4 \\
2\end{array}$ & $\begin{array}{l}25052.9 \\
25027.0 \\
24992.3 \\
24981.17 \\
24622.58\end{array}$ & $\begin{array}{l}+0.1 \\
+0.1 \\
+0.1 \\
-0.01 \\
+0.06\end{array}$ & $\begin{array}{l}22535_{01 / 3}-47588_{11 / 2}^{\circ} \\
23803_{31 / 2}-48830_{31 / 2}^{\circ} \\
28377_{51 / 2}-53869_{41 / 2}^{\circ} \\
33910_{51 / 2}-58891_{51 / 2}^{\circ} \\
28491_{11 / 2}^{\circ}-5313_{21 / 2}^{\circ}\end{array}$ & 6 \\
\hline $\begin{array}{l}\text { 4069. } 61 \\
4081.294 \\
4114.19 \\
4144.41 \\
4146.62\end{array}$ & $\begin{array}{l}1 \\
4 \\
1 \\
1 \\
-?\end{array}$ & $\begin{array}{r}10 \\
2 \\
1\end{array}$ & $\begin{array}{l}24565.4 \\
24495.12 \\
24299.3 \\
24122.1 \\
24109.2\end{array}$ & $\begin{array}{l}-0.3 \\
+0.07 \\
-0.08 \\
-0.1 \\
-0.1\end{array}$ & $\begin{array}{c}26929_{51 / 2}-z^{6} \mathrm{~F}_{51 / 2}^{\circ} \\
a^{4} \mathrm{D}_{11 / 2}-z^{6} \mathrm{~F}_{11 / 2}^{\circ} \\
22194_{31 / 2}-z^{6} \mathrm{~F}_{41 / 2}^{\circ} \\
22503_{11 / 2}-46625_{01 / 2}^{\circ} \\
25045_{01 / 2}-491540^{11 / 2}\end{array}$ & 6 \\
\hline $\begin{array}{l}4157.030 \\
4165.60 \\
4175.58 \\
4257.82 \\
4262.75\end{array}$ & $\begin{array}{l}2 \\
1 \\
5 \\
1 \\
1\end{array}$ & $\begin{array}{l}5 \\
2 \\
5\end{array}$ & $\begin{array}{l}\text { 24048. } 86 \\
23999.4 \\
23942.0 \\
23479.6 \\
23452.4\end{array}$ & $\begin{array}{l}+0.11 \\
+0.2 \\
0.0 \\
-0.2 \\
0.0\end{array}$ & $\begin{array}{c}18000_{31 / 2}-z^{6} \mathrm{~F}_{21 / 2}^{\circ} \\
20455_{11 / 2}-44455_{01 / 2}^{\circ} \\
a^{4} \mathrm{D}_{11 / 2}-z^{2} \mathrm{~S}_{01 / 2}^{\circ} \\
24804_{31 / 2}-48284^{\circ} 2^{11 / 2} \\
25672_{21 / 2}-49124^{\circ} 3^{1 / 2}\end{array}$ & \\
\hline 4263. 81 & 1 & & 23446.6 & 0. 0 & $23046_{31 / 2}-z^{6} \mathrm{~F}_{41 / 2}^{\circ}$ & \\
\hline 4289. 28 & 2 & 2 & 23307. 4 & $\left\{\begin{array}{r}0.0 \\
+0.2\end{array}\right.$ & $\begin{array}{l}28186_{61 / 2}-z{ }^{0}{ }^{\circ} 51 / 2 \\
18990_{11 / 2}-42298^{\circ 1 / 2}\end{array}$ & \\
\hline $\begin{array}{l}\text { 4298. } 26 \\
4323.392 \\
4335.58\end{array}$ & $\begin{array}{l}1 \\
1 \\
1\end{array}$ & $\begin{array}{l}6 \\
6\end{array}$ & $\begin{array}{l}23258.7 \\
23123.49 \\
23058.5\end{array}$ & $\begin{array}{l}+0.1 \\
+0.04 \\
\quad 0.0\end{array}$ & $\begin{array}{l}23234_{41 / 2}-z^{6} \mathrm{~F}_{41 / 2}^{\circ} \\
25209_{41 / 2}-48332_{51 / 2}^{\circ} \\
18990_{11 / 2}-z^{6} \mathrm{~F}_{21 / 2}^{\circ}\end{array}$ & \\
\hline $\begin{array}{l}\text { 4342. } 40 \\
4343.20 \\
4348.108 \\
4361.534 \\
4393.80\end{array}$ & $\begin{array}{l}2 \\
20 A \\
8 A \\
1\end{array}$ & $\begin{array}{r}2 \\
5 \\
25 \\
3 \\
2\end{array}$ & $\begin{array}{l}\text { 23022. } 3 \\
23018.0 \\
22992.05 \\
22921.28 \\
22753.0\end{array}$ & $\begin{aligned} & 0.0 \\
+ & 0.1 \\
+ & 0.08 \\
+ & 0.06 \\
+ & 0.1\end{aligned}$ & $\begin{array}{c}26158_{41 / 2}-49181_{41 / 2}^{\circ} \\
22535_{01 / 2}-45553^{\circ}{ }_{11 / 2} \\
a^{4} \mathrm{D}_{01 / 2}-z^{6} \mathrm{~F}_{01 / 2}^{\circ} \\
22535_{01 / 2}-45457^{\circ 1 / 2} \\
19637_{21 / 2}-42390_{31 / 2}^{\circ}\end{array}$ & $\begin{array}{l}\text { res } \\
\text { res }\end{array}$ \\
\hline
\end{tabular}


TABLE 3. Classified lines of $\mathrm{W}$ II-Continued

\begin{tabular}{|c|c|c|c|c|c|c|}
\hline \multirow{2}{*}{$\begin{array}{c}\text { Wavelength } \\
(\AA)\end{array}$} & \multicolumn{2}{|c|}{ Intensity } & \multicolumn{2}{|c|}{ Wave number $\left(\mathrm{cm}^{-1}\right)$} & \multirow{2}{*}{ Combination } & \multirow{2}{*}{$\begin{array}{c}\text { Zeeman } \\
\text { effect }\end{array}$} \\
\hline & Arc & Spark & Observed & $O-C$ & & \\
\hline \multicolumn{7}{|l|}{ Air } \\
\hline 4396.88 & 2 & 2 & 22737. 0 & $\left\{\begin{array}{r}-0.2 \\
0.0\end{array}\right.$ & $\begin{array}{l}22139_{21 / 2}-z^{6} \mathrm{~F}_{31 / 2}^{\circ} \\
30633_{41 / 2}-53369_{41 / 2}^{\circ}\end{array}$ & \\
\hline $\begin{array}{l}4421.836 \\
4438.92 \\
4468.72 \\
4508.50\end{array}$ & $15 A$ & $\begin{array}{l}3 \\
2 \\
1 \\
2\end{array}$ & $\begin{array}{l}22608.69 \\
22521.7 \\
22371.5 \\
22174.1\end{array}$ & $\begin{array}{r}+0.03 \\
0.0 \\
-0.1 \\
+0.1\end{array}$ & $\begin{array}{l}24804_{31 / 2}-47413_{21 / 2}^{\circ}-46612_{31 / 2}^{\circ} \\
34091_{41 / 2}-566175_{31 / 2}^{\circ} \\
23803_{31 / 2}-46832_{51 / 2}^{\circ} \\
26158_{41 / 2}-4839\end{array}$ & \\
\hline 4542. 144 & 1 & 2 & 22009. 86 & $\left\{\begin{array}{l}-0.21 \\
+0.15\end{array}\right.$ & $\begin{array}{l}25169_{11 / 2}-4^{7} 179_{11 / 2}^{\circ} \\
20039_{31-{ }^{6}} \mathrm{~F}^{\circ 11}\end{array}$ & \\
\hline $\begin{array}{l}4554.045 \\
4604.576 \\
4658.24 \\
4665.76\end{array}$ & $\begin{array}{l}1 \\
1 \\
2 \\
4 A\end{array}$ & $\begin{array}{l}2 \\
3 \\
2 \\
3\end{array}$ & $\begin{array}{l}\text { 21952. } 35 \\
21711.44 \\
21461.3 \\
21426.74\end{array}$ & $\begin{array}{r}+0.13 \\
+0.23 \\
+0.14 \\
+0.19 \\
+0.06\end{array}$ & $\begin{array}{l}22503_{11 / 2}-44455_{01 / 2}^{\circ} \\
23046_{31 / 2}-44758_{41 / 2}^{\circ} \\
23450_{21 / 2}-44911_{11 / 2}^{\circ} \\
23450_{21 / 2}-z^{6} \mathrm{~F}_{31 / 2}^{\circ}\end{array}$ & \\
\hline $\begin{array}{l}4691.74 \\
4718.91 \\
4768.25 \\
4782.35 \\
4864.565\end{array}$ & $\begin{array}{l}1 \\
1 \\
1 \\
1 \\
2\end{array}$ & $\begin{array}{l}2 \\
3 \\
2 \\
1 \\
3\end{array}$ & $\begin{array}{l}21308.1 \\
21185.4 \\
20966.2 \\
\text { 20904. } 4 \\
\text { 20551. } 08\end{array}$ & $\begin{array}{l}+0.1 \\
-0.1 \\
+0.1 \\
+0.1 \\
+0.10\end{array}$ & $\begin{array}{l}23046_{31 / 2}-44354^{\circ} 11 / 2 \\
25169_{11 / 2}-46355_{21 / 2}^{\circ} \\
25209_{41 / 2}-4615_{3112}^{\circ} \\
23450_{21 / 2}-4435421 / 2 \\
23803_{31 / 2}-4435421 / 2\end{array}$ & \\
\hline $\begin{array}{l}4959.33 \\
5021.324 \\
5104.427 \\
5114.59 \\
5131.83\end{array}$ & $\begin{array}{l}3 \\
5 \\
6 \\
3 \\
2\end{array}$ & $\begin{array}{l}2 \\
2 \\
6 \\
3 \\
2\end{array}$ & $\begin{array}{l}20158.4 \\
19909.51 \\
19585.37 \\
19546.46 \\
19480.8\end{array}$ & $\begin{array}{l}+0.2 \\
+0.03 \\
+0.01 \\
+0.07 \\
-0.1\end{array}$ & $\begin{array}{l}22139_{21 / 2}-42298_{11 / 2}^{\circ} \\
221399_{21 / 2}-z^{6} \mathrm{~F}_{21 / 2}^{\circ} \\
18990_{11 / 2}-z^{2} \mathrm{~S}_{01 / 2}^{\circ} \\
22503_{11 / 2}-z^{6} \mathrm{~F}_{21 / 2}^{\circ} \\
20455_{11 / 2}-39936_{21 / 2}^{\circ}\end{array}$ & \\
\hline $\begin{array}{l}5214.41 \\
5219.04 \\
5260.92 \\
5278.38 \\
5304.17\end{array}$ & $\begin{array}{r}2 \\
3 \\
4 \\
8 \\
10\end{array}$ & $\begin{array}{l}? \\
? \\
2 \\
4\end{array}$ & $\begin{array}{l}19172.3 \\
19155.3 \\
19002.8 \\
18939.9 \\
18847.8\end{array}$ & $\begin{array}{l}+0.1 \\
-0.1 \\
+0.2 \\
+0.1 \\
+0.1\end{array}$ & $\begin{array}{l}19404_{01 / 2}-z^{2} \mathrm{~S}_{01 / 2}^{\circ} \\
23234_{41 / 2}-42390_{31 / 2}^{\circ} \\
23046_{31 / 2}-z^{6} \mathrm{~F}_{21 / 2}^{\circ} \\
23450_{21 / 2}-42390_{31 / 2}^{\circ} \\
23450_{21 / 2}-42298_{11 / 2}^{\circ}\end{array}$ & \\
\hline 5353.677 & 5 & 3 & 18673.54 & +0.06 & $20455_{11 / 2}-z^{6} \mathrm{~F}_{11 / 2}^{\circ}$ & \\
\hline 5375. 103 & 3 & 2 & 18599. 12 & $\left\{\begin{array}{l}-0.24 \\
+0.17\end{array}\right.$ & $\begin{array}{l}26158_{41 / 2}-4+758_{41 / 2}^{\circ} \\
23450_{21 / 2}-z^{6} \mathrm{~F}_{21 / 2}^{\circ}\end{array}$ & \\
\hline $\begin{array}{l}5410.89 \\
5597.056 \\
5698.47\end{array}$ & $\begin{array}{l}2 \\
3 \\
1\end{array}$ & $\begin{array}{l}3 \\
1\end{array}$ & $\begin{array}{l}\text { 18476. } 12 \\
17861.63 \\
17543.7\end{array}$ & $\begin{array}{l}-0.01 \\
+0.06 \\
+0.1\end{array}$ & $\begin{array}{l}34091_{41 / 2}-52567^{\circ 1 / 2} \\
28631_{31 / 2}-z \mathrm{~F}_{41 / 2}^{\circ} \\
28631_{31 / 2}-4615^{\circ}{ }_{31 / 2}^{\circ}\end{array}$ & \\
\hline $\begin{array}{l}5821.00 \\
5884.32 \\
5964.48 \\
6024.64 \\
6219.77\end{array}$ & $\begin{array}{l}4 A \\
2 \\
3 \\
2 \\
2\end{array}$ & $\begin{array}{l}1 \\
2 \\
5 \\
5 \\
8\end{array}$ & $\begin{array}{l}\text { 17174. } 42 \\
16989.6 \\
16761.28 \\
16593.91 \\
16073.32\end{array}$ & $\begin{array}{l}+0.03 \\
+0.2 \\
+0.01 \\
+0.30 \\
+0.06\end{array}$ & $\begin{array}{l}18990_{11 / 2}-z^{6} \mathrm{~F}_{01 / 2}^{\circ} \\
22139_{21 / 2}-z^{6} \mathrm{~F}_{11 / 2}^{\circ} \\
19404_{01 / 2}-z^{6} \mathrm{~F}_{01 / 2}^{\circ} \\
22535_{01 / 2}-z^{6} \mathrm{~F}_{11 / 2}^{\circ} \\
22503_{11 / 2}-z^{2} \mathrm{~S}_{01 / 2}^{\circ}\end{array}$ & \\
\hline
\end{tabular}

I gratefully acknowledge the assistance of Dr. C. C. Kiess, who supplied most of the spectrograms, and of Mrs. Isabel Murray, who carefully prepared press-copy of the tables.

\section{References}

[1] O. Laporte, Regelmässigkeiten im Bogenspektrum des Wolframs, Naturwissenschaften 13, 627-628 (1925).

[2] O. Laporte and J. E. Mack, The spectrum of neutral tungsten, W I. Phys. Rev. 63, 246-297 (1943).

[3] D. D. Laun, Preliminary lists of terms for the are and spark spectra of tungsten, J. Res. NBS 21, 207-224 (1938) RP 1125.

[4] D. D. Laun, Arc spectrum of tungsten: University of Chicago, Dissertation; University of Chicago Libraries, Chicago, Ill. (1934).
[5] C. D. Coleman, W. R. Bozman, and W. F. Meggers, Table of wavenumbers, NBS Mono. 3, U.S. Government Printing Office, Washington: 1960 .

[6] Charlotte E. Moore, Atomic energy levels, NBS Cir. 467, vol. III, 161-163 (1958).

[7] E. Back and A. Landé, Zeemaneffekt und Multiplettstruktur der Spectrallinien, 168-169 (Julius Springer, Berlin, 1925).

[8] W. F. Meggers, C. H. Corliss, and B. F. Scribner, Tables of spectral-line intensities, NBS Mono. 32, pt. 1, 402-415 (U.S. Government Printing Office, Washington, 1961).

[9] C. C. Kiess, C. J. Humphreys, and D. D. Laun, Preliminary description and analysis of the first spectrum of uranium, J. Res. NBS 37, 57-72 (1946) RP 1729.

(Paper 68A2-270) 


\section{Publications of the National Bureau of Standards*}

\section{Selected Äbstracts}

Gaussian wave functions for polyatomic molecules: integral formulas, M. Krauss, J. Res. 68B (Math. and Math. Phys.), No. 1, 35-41 (Jan.-Mar. 1894).

Explicit formulas are given for the molecular coulomb integrals that arise for all Gaussian basis functions. These results should expedite computational efforts with these basis functions.

A Fabry-Perot spectrometer for high-resolution spectroscopy and laser work, K. D. Mielenz, R. B. Stephens, and K. F. Nefflen, J. Res. 68C (Eng. and Instr.), No. 1, 1-6 (Jan.-Mar. 1964)

A Fabry-Perot spectrometer with a piezoelectric spacer was constructed to record with a recorder, or display with an ocilloscope, the fine structure of the Haidinger fringes. The spectrometer is of the fixed spacer design, which provides great stability of adjustment. It was used to record the Zeeman splitting of the green line of $\mathrm{Hg} 198$, as well as to exhibit, on the oscilloscope screen, the multimode output signal of a He-Ne gas laser.

A laser with a multihole diaphragm, T. Morokuma, J. Res. NBS 68C (Eng. and Instr.), No. 1, 25-34 (Jan.-Mar. 1964). The properties of a laser with a multihole diaphgram were both theoretically and experimentally examined. This laser may be called a multibeam laser. Laser action was observed in the optical paths which were defined by the position of the holes and the cavity configuration. Interference fringes were observed on one of the cavity mirrors. A wavelength dependent interaction among the beams was observed. It is believed that the wavelength of a beam can be stabilized by the intensities of the other beams. A possible method will be proposed for the stabilization.

Principles of cryometric impurity determination as applied to samples of small sizes, C. P. Saylor and G. S. Ross, J. Res, NBS 68C (Eng. and Instr.), No. 1, 35-39 (Jan.-Mar. 1964). A consideration of the factors that tend to prevent attainment of thermal and thermodynamic equilibrium during thermometric methods of purity analysis suggests that the problems largely originate from spontaneously introduced inequalities in composition. During either a freezing or melting cycle, the solid phase settles in the liquid. This results in a greater average purity in the bottom than in the upper part of the vessel. The effects would be particularly strong during a melting sequence where the liquid released by melting of the solid would provide the compositional environment for the establishemnt of final temperature. This hypothesis has been tested by the design of a new cell for small samples. The design incorporates use of small gold pans to hold solid and liquid in close contact. The results are comparable in precision to those from conventional time-temperature curves with much larger samples, a condition not ordinarily possible with small samples.

Reference tables for thermocouples of iridium-rhodium alloys versus iridium, G. F. Blackburn and F. R. Caldwell, $J$. Res. NBS 68C (Eng. and Instr.), No. 1, 41-59 (Jan.Mar. 1964).

The program at the National Bureau of Standards to establish reference tables of temperature versus emf for thermocouples of iridium-rhodium alloys versus iridium has been extended to cover all three of the currently used thermocouples of this type. In addition to the values published in 1962 for the $40 \mathrm{Ir}-60 \mathrm{Rh}$ versus Ir thermocouples, tables now are available for thermocouples of $60 \mathrm{Ir}-40 \mathrm{Rh}$ and $50 \mathrm{Ir}-50 \mathrm{Rh}$ versus Ir. These tables give emfs for temperatures in degrees
Celsius from 0 to $2150{ }^{\circ} \mathrm{C}$ and in degrees Fahrenheit from 32 to $3900{ }^{\circ} \mathrm{F}$, and temperatures in these units with emf in millivolts as the argument.

In addition to the reference tables for these thermocouples, temperature-emf relationships are presented for other alloys containing 10, 25, 75, and 90 percent iridium versus iridium. It appears from the information obtained on all of the alloy versus iridium combinations tested that the $50 \mathrm{Ir}-50 \mathrm{Rh}$ alloy versus iridium gives about the maximum thermal emf (12.2 mv at $2150{ }^{\circ} \mathrm{C}$ ), and as a result may provide the optimum thermocouple combination of this type.

A simplified theory of diffraction at an interface separating two dielectrics, J. Kane and S. N. Karp, Radio Sci. J. Res. NBS/USNC-URSI 68D No. 3, 303-310 (Mar. 1964).

Many electromagnetic problems involving more than one dielectric medium are not susceptible of an exact solution, when appropriate boundary conditions are considered. The purpose of the present paper is to formulate a new boundary condition, which is capable of leading to mathematically tractable problems, with limited sacrifices in accuracy.

Propagation of plasma waves in a "spoke-wheel" magnetic field, R. L. Liboff, Radio Sci. J. Res. NBS/USNC-URSI 68D No. 3, 325-331 (Mar. 1964).

A study of the cold plasma cylindrical waves that may propagate in a specific type of two-dimensional magnetic field is initiated in this paper. The plasma is assumed to be of uniform density and collisionless, and a "spoke-wheel" magnetic field is considered which is both anisotropic and inhomogeneous (varying as the inverse radius), as defined in the text. Perturbation series solutions are obtained for the first Fourier component of the electric field for the four extreme cases: large and small magnetic field; large and small plasma densities.

Compilation of the melting points of the metal oxides, S. J Schneider, NBS Mono. 68 (Oct. 10, 1963), 25 cents.

A compilation has been made of the melting points of 70 metal oxides published prior to January 1963. Both the original melting point and the equivalent value based on the International Practical Temperature Scale of 1948 are presented. Included in the survey is information on pertinent experimental details such as the method of temperature measurement, purity, furnace type, and environmental conditions.

Calibration and test services of the National Bureau of Standards, NBS Misc. Publ. 250 (Nov. 22, 1963), 70 cents.

This publication is a listing of the numerous calibration and testing services provided to science and industry by the National Bureau of Standards. The Bureau promotes accuracy and uniformity of measurement through its program of measurement services, including the calibration and testing of standards and standard instruments. An up-to-date listing of the Bureau's calibration and test fee schedules was printed in recent issues of the Federal Register, with a large proportion of the fees being changed at that time. This publication contains all of this material, but in larger, more legible type, and fully indexed. Besides listing all NBS calibration services and their cost, the publication includes a statement of the Bureau's statutory functions, testing policy, and routine for securing the Bureau's calibration and test services-from original request to the reporting and use of test results.

A comparison of two melting-pressure equations constrained to the triple point using data for eleven gases and three metals, R. D. Goodwin and L. A. Weber, NBS Tech. Note 184 (Oct. 9, 1963), 25 cents. 
Parameters have been determined by a least-squares method for the reduced Simon equation and for a new, empirical melting equation using data for $\mathrm{H}_{2}, \mathrm{D}_{2}, \mathrm{~T}_{2}, \mathrm{Ne}, \mathrm{Ar}, \mathrm{Kr}$, $\mathrm{Xe}, \mathrm{N}_{2}, \mathrm{O}_{2}, \mathrm{CO}_{2}, \mathrm{H}_{2} \mathrm{O}, \mathrm{Na}, \mathrm{K}$, and $\mathrm{Hg}$. The new equation $\left(P-P^{3}\right) /\left(T-T^{3}\right)=A \exp (-\alpha / T)+B T$, represents experimental data with essentially the same accuracy as the Simon equation. It provides a sensitive difference method for graphical examination of data.

Carbon resistors for cryogenic liquid level measurements, R. C. Muhlenhaupt and P. Smelser, NBS Tech. Note 200 (Oct. 8, 1963), 25 cents.

Data are shown in graphical form. One set of plots presents resistance ratio $R_{G} / R_{L}$ as a function of "warming up" time at various levels of constant power dissipation. A second set of plots presents resistance ratio $R_{L} / R_{O}$ as a function of nominal resistance at various levels of constant power dissipation.

The use of the data and the design of a practical liquid level indicator are discussed in the appendix.

Absolute calibration of the National Bureau of Standards photoneutron source: III. Absorption in a heavy water solution of manganous sulfate, R. H. Noyce, E. R. Mosburg, Jr., S. B. Garfinkel, and R. S. Caswell, J. Nucl. Eng. 17, No. $7,313-318(1963)$.

The neutron emission rate for the National Bureau of Standards radium-beryllium $(\gamma, n)$ standard source (NBS-I) has been redetermined by a relative comparison to an antimony beryllium source which had been calibrated absolutely in a heavy water manganous sulfate solution through an indirect method involving $4 \pi \beta-\gamma$ coincidence counting of the induced $\mathrm{Mn}^{56}$ activity. Correction was made for absorption of neutrons by other elements present in the bath. The emission rate of NBS-I was found to be $1.25_{2} \times 10^{6}$ neutrons per second, as of June 1961 with an uncertainty of 1 percent. This measurement, when suitably averaged with previous determinations (DeJuren, Padgett, and Curtiss, 1955; and DeJuren and Chin, 1955), yields the best value $1.257 \times 10^{6}$ neutrons per second with an uncertainty of 1 percent.

Heating rate as a test of adiabatic calorimeters and the heat capacity of $\alpha$ alumina, E. D. West, Trans. Faraday Soc. 59, No. 489, pt. 9, 2200-2203 (Sept. 1963).

The constancy of heat capacities measured in an adiabatic calorimeter at different heating rates does not demonstrate the absence of heat leak errors due to departures of the surface temperature from the "isothermal" condition. On the con trary, the total heat exchange between the calorimeter and its shield is virtually independent of the heating rate. The test tends to obscure a real source of error due to the variation in the total heat exchange between experiments with the empty and the full calorimeter.

A new standard of spectral irradiance, R. Stair, W. E Schneider, and J. K. Jackson, Appl. Opt. 2, No. 11, 1151-115. (Nov. 1963)

The National Bureau of Standards has made available a new standard of spectral irradiance in the form of a 200-watt quartz-iodine lamp with a coiled-coil tungsten filament operating at about $3000^{\circ} \mathrm{K}$ and calibrated over the spectral range of 0.25 to 2.6 microns. The calibration of this standard is based upon the radiance of a blackbody as defined by the Planck law of radiation since it was done by comparison. with the NBS standards of spectral radiance, of luminous intensity, and of total irradiance, each of which was established through the use of blackbodies. This standard is used without auxiliary optics. Because of its small physical size and high operating temperature, relatively high spectral irradiances may be obtained through its use.

Intercomparisons of the standard thermal-neutron flux density of the National Bureau of Standards, W. M. Murphey and J. Chin, (Proc. Symp. Neutron Detection, Dosimetry and Standardization, International Atomic Energy Agency, Atomic Energy Research Establishment, Harwell, England, Dec. 10 14, 1062), Book, Neutron Dosimetry II, 513-521 (International Atomic Energy Agency, Vienna, 1963)

The standard thermal neutron flux maintained at the $\mathrm{Na}$ - tional Bureau of Standards since 1951 consists of two radiumberyllium $(\alpha, n)$ sources in a geometry of lead, paraffin and carbon. The flux has been absolutely measured twice by NBS; once in terms of the absorption cross section of boron, and once in terms of the absorption cross section of gold $(1,2)$. A neutron flux with a cadmium ratio of about 7 (for 40 $\mathrm{mg} / \mathrm{cm}^{2}$ gold foils and 0.040 inch cadmium covers) and a conventional thermal neutron flux density of 4231 (Apr. 62) neutrons per $\mathrm{cm}^{2}$ per second was obtained. It has also been absolutely measured twice through the intercomparison program; once by NPL, UK and once by PTB, Federal Republic of Germany.

The agreement between most laboratories at present is about $1.5 \%$. This is in addition to the uncertainty in the absolute measurement which is about $1.5 \%$ or more for each laboratory. Intercomparisons of NBS with CEA, France; Oak Ridge National Laboratory, USA; Electrotechnical Laboratory, Japan; National Physical Laboratory, UK; and PhysikalischTechnische Bundesanstalt, Federal Republic of Germany will be shown.

Interpretation of $p \mathrm{H}$ measurements in alcohol-water solvents, R. G. Bates, M. Paabo, and R. A. Robinson, J. Phys. Chem. 6\%, 1833-1838 (1963).

The operational $p \mathrm{H}$ numbers for solutions in alcohol-water solvents obtained from the e.m.f. of the cell with hydrogen (or glass) electrode, salt bridge, and calomel reference electrode, standardized with aqueous buffer standards, are subject to no simple interpretation. Because of the indeterminate potential at the junction Soln. $\mathrm{X}\left(\right.$ alc. $\left.-\mathrm{H}_{2} \mathrm{O}\right) \mid \mathrm{KCl}$ bridge (aq.), they do not lie on a conventional scale of hydrogen ion activity referred either to the aqueous standard state $\left(p a_{\mathrm{H}}\right)$ or to the standard state in the alcoholic medium $\left(p a_{\mathrm{H}}^{*}\right)$. Values of $\bar{E}_{j}+\log _{m} \gamma_{\mathrm{Cl}}$ (where $\bar{E}_{j}$ is the liquid-junction potential expressed in $p \mathrm{H}$ units and ${ }_{m} \gamma_{\mathrm{Cl}}$ is the primary medium effect of chloride ion) were found to be as constant for different buffer solutions in alcoholic solvents of fixed composition as for the strictly aqueous medium. Inasmuch as $m \gamma_{\mathrm{Cl}}$ at a given temperature is dependent only on solvent composition, the liquid-junction potential must therefore also be nearly constant for a given solvent medium. Correction terms $\bar{E}_{i}-\log \gamma_{\mathrm{H}}=\delta$ have been calculated, and it is shown that $p \mathrm{H}-\delta$ closely approximates $p \alpha_{\mathrm{H}}^{*}$ under optimum conditions of measurement. An operational scale of $p \mathrm{H}^{*}$ (which is related to $p a_{\mathrm{H}}^{*}$ in the same way that $p \mathrm{H}$ is related to $\left.p a_{\mathrm{H}}\right)$ is described. Values of $p a_{\mathrm{H}}^{*}$ were obtained for 12 solutions in methanolwater solvents ( 0 to $68.1 \mathrm{wt} . \% \mathrm{MeOH}$ ) and for 3 solutions in ethanol-water solvents (0 to $100 \%$ EtOH).

Short-duration visible afterglow in helium, A. L. Schmeltekopf, Jr., and H. P. Broida, J. Chem. Phys. 39, 1261-1268 (Sept. 1963).

A bright, reproducible afterglow which consists of helium atomic and molecular emissions has been observed in the products of an electrical discharge in a deLaval nozzle clearly separated from the discharge. This afterglow has been observed at pressures from 0.4 to $20 \mathrm{~mm} \mathrm{Hg}$ and with flows from 10 to $1000 \mathrm{~cm}^{3} / \mathrm{sec}$ NTP. At the low pressure the existence of the afterglow is extremely sensitive to small changes in pressure. Metastable He atom concentration decreases after the discharge and then increases in the region of afterglow emission. The afterglow is very sensitive to impurities greater than a few parts in $10^{5}$ and emission intensity is reduced by the presence of an rf field, possibly because heating the electrons retards recombination.

Infrared transmission of the atmosphere to solar radiation, D. M. Gates and W. J. Harrop, Appl. Opt. 2, 887-898 (Sept. 1963)

Infrared solar spectrum observations taken on 12 January 1955 in Denver, Colorado, with a double pass $\mathrm{NaCl}$ prism spectrometer have been analyzed for transmission coefficients for the "selective" absorption and for the "continuum" extinction. The analysis was performed over the range 1.0 to $12.5 \mu$. The goodness of fit for the average transmission for a random band model and for a regular band model is given for each of 203 data points distributed throughout the spectrum. 
Some topics in quantum statistics. The Wigner function and transport theory, H. Mori, I. Oppenheim, and J. Ross, Book, Studies in Statistical Mechanics, ed. DeBoer and Uhlenbeck, I, Pt. C, 218-298 (North Holland Publ. Co., Amsterdam, the Netherlands, 1962).

This article is devoted to a discussion of Wigner functions and their application in quantal statistical mechanics, as well as some recent work in the theory of non-equilibrium systems. An analysis of these systems also requires the inclusion of some other topics: scattering theory, representative ensembles, and factors influencing the derivation of a transport equation for irreversible phenomena. We begin with the description of a quantum mechanical system. In Section I we first present some definitions and then discuss scattering theory. The description of equilibrium systems is given in Section II with primary emphasis on the construction of ensembles. A basis for the discussion of non-equilibrium systems is given in Section III and the construction of ensembles are treated in some detail. In Section IV, a derivation of a transport equation for non-uniform systems (the Maxwell-Boltzmann equation) is outlined. Section $\mathrm{V}$ is devoted to an alternative approach to transport theory for gases and 'liquids (the correlation function method).

Thermodynamic study of the thorium phosphide with a mass spectrometer, J. Efimenko and K. A. Gingerich, Proc. Symp. Thermodynamics of Nuclear Materials, Vienna, May 21-25, 1963, pp. 47\%-486 (International Atomic Energy Agency, Vienna, A ustria, Sept. 1962).

For the thorium-phosphorous system the ion intensity variation with temperature over the range $1100^{\circ}-2250^{\circ} \mathrm{K}$ and composition range $\mathrm{ThP}_{1.3}-\mathrm{ThP}_{0.6}$ has been obtained for the phosphorus vapor species. No molecules containing both thorium and phosphorus have been observed in the vapor below $2245^{\circ} \mathrm{K}$. With the aid of a silver calibration the ion intensities have been related to the partial pressures of the effusing phosphorus vapor species, $\mathrm{P}, \mathrm{P}_{2}$, and $\mathrm{P}_{4}$. From these data the "apparent" molar free energies of formation have been obtained with reference to gaseous $\mathrm{P}_{2}$ for different compositions of the solid and the dissociation energy for $\mathrm{P}_{2}$ has been computed. The vaporization of the thorium phosphides occurs incongruently by decomposition into gaseous phosphorus and a condensed phase of lower phosphorus content. The rate of phosphorus loss from the $\mathrm{Th}_{3} \mathrm{P}_{4}$ phase appears to be diffusion controlled. There is a marked increase of thermodynamic stability with decreasing phosphorus content within the homogeneity range of the lower phosphide. The phase boundaries of the lower phosphide have been estimated to correspond to $\mathrm{Th}_{0.7}$ and $\mathrm{Th}_{0.9}$. The thermodynamic stability of the thorium phosphides is compared with that of selected lower phosphides of transition metals.

Low energy levels of neutral cerium (Ce I), W. C. Martin, J. Opt. Soc. Am. 53, No. 9, 1047-1050 (Sept. 1963).

The first results of a continuing analysis of the Ce I spectrum show the ground level to be $4 f 5 d 6 s^{2}{ }^{1} \mathrm{G}_{4}^{\circ}$, with $g_{J}=0.945$. Of twelve other low odd levels for which the energies, J- and $g$-values are given, six have been assigned to $4 f 5 d 6 s^{2}$ and three to $4 f 5 d^{2} 6 s$.

A study of stress relaxation with finite strain, B. Bernstein, E. A. Kearsley, and L. J. Zapas, Trans. Soc. Rheology VII, $391-410(1963)$.

Two simple types of constitutive equations appropriate to materials exhibiting elasticity are presented, one of a basic solid nature and one of a basic fluid nature. The predictions of the equations for a stress relaxation experiment are worked out and compared to the data from some experiments on various elastomers. The fluid theory is shown to be most appropriate in a certain sense.

Measurement of weak magnetic fields by optical pumping methods, P. L. Bender, Bull. Ampere 9e, Pt. II, 261-628 (1960)

Three new types of magnetometer, the de alkali vapor magnetometer, the self-oscillating alkali vapor magnetometer, and the helium magnetometer, all based on optical pumping, are compared. Practical limitations and performance in various environmental circumstances are considered.
Three-body bound state in $\mathrm{He}^{4}$, J. M. Blatt, J. N. Lyness, and S. Y. Larsen, Phys. Rev. 131, No. 5, 2131-2132 (Sept. 1, 1963).

The binding energy of three $\mathrm{He}^{4}$ atoms, subject to pair forces, is investigated numerically for a number of proposed intermolecular potentials and for a series of square wells. For some potentials a 3 -body bound state is found, for others not. However, a direct correlation is found between 2-body and 3 -body binding. The need for more accurate experimental data is discussed.

Momentum autocorrelation functions and energy transport in harmonic crystals containing isotopic defects, R. J. Rubin, Phys. Rev. 131, 964 (1963).

In this paper we review and extend the investigation of the effect of isotopic impurities on two statistical dynamical properties of harmonic erystals: the decay of the momentum autocorrelation function and the transport of energy. A spectral representation is obtained for the momentum autocorrelation functions. The spectral density is directly related to the normal mode frequency distribution of the crystal; the recent investigations of the classical momentum autocorrelation function in a perfect one-dimensional crystal and a one-defect crystal are discussed as special cases of this general Wiener-Khinchin formula. The corresponding quantum mechanical autocorrelation function is also treated. A formal relation involving the average momentum autocorrelation function of an isotopically disordered crystal and the frequency spectrum of the crystal is derived. The energy transport property is studied in terms of the timedependent dispersion of the momentum of a lattice particle when the crystal is characterized initially by a spatially nonuniform temperature. The local temperature, which is related to the momentum dispersion, is studied analytically in the case of the perfect one-dimensional crystal and the one-defect one-dimensional crystal. The local temperature is studied numerically with the aid of an IBM 7090 for several isotopically disordered one-dimensional crystals.

Galvanostalametry, a new technique based on the negative pressure of liquids, for investigating electrochemical phenomena at an electrode, J. Sligh and A. Brenner, J. Electrochem. Soc. 110, No. 11, 1136-1142 (Nov. 1963).

A procedure based on the negative pressure of water has been used as a sensitive indicator for the study of electrochemical phenomena. The apparatus consisted of a vertical glass tube, closed at the upper end, which was evacuated and filled with an electrolyte. The latter remained suspended in the column in a metastable state of tension. The column of electrolyte was dropped by producing a minute amount of electrolysis between an indicator electrode sealed in the top of the column and a companion electrode which could also be at the top or in the reservoir at the bottom of the column. The time or current required to drop the column was used for making the following experiments and/or observations: $(A)$ The decomposition potential of water was determined by observing the lowest applied voltage required to drop the column. (B) The time required to drop the column (on passage of a constant current) was proportional to the concentration of iodide ion in the electrolyte. (C) The current required to drop the column was proportional to the area of the indicator electrode, regardless of the irregularity of its shape. (D) The formation of the gas required to drop the column occurred in less than $15 \mu$ sec after closing the circuit.

Electrical resistance-strain characteristics of thin evaporated metal films, R. L. Parker and A. Krinsky, J. Appl. Phys. 34, No. 9, 2700-2708 (Sept. 1963).

The electrical resistance-strain coefficients of thin evaporated films of Aluminum, Gold, Cobalt, Nickel, Palladium, Platinum, Antimony, and Tellurium have been measured. The coefficients (strain-sensitivity coefficient or "gaugefactor" $\gamma$ ) depend on the specific resistance $R_{s}$ of the films. For relatively thick films (small $R_{s}$ ), $\gamma$ tends to approach the bulk value $\gamma_{b}$; for relatively thin films (large $R_{s}$ ), $\gamma$, may be many times greater than $\gamma_{b}$; for films of intermediate thickness $\gamma$ has a minimum value. The resistance change was found to be proportional to strain for all the above films except those of $\mathrm{Ni}$ and $\mathrm{Sb}$. Some possible mechanisms 
for the observed behavior are discussed; it was found that free-path effects are predominant in films of intermediate thickness, and that the tunnelling conduction mechanism plays an important role in producing the high strain-sensitivity of very thin (high $R_{s}$ ) films of separated particles.

\section{Other NBS Publications}

\section{J. Res. NBS 68B (Math. and Math. Phys.), No. 1 (Jan.- Mar. 1964) 75 cents.}

A note on a generalized elliptic integral. G. H. Weiss.

An asymptotic expansion for the multivariate normal distribution and Mills' ratio. H. Ruben.

Calculation of certain multiple generating functions. G. H. Weiss.

Some infinite sums involving zeros of $J_{0}(x)$. L. F. Epstein.

Optimal matchings and degree-constrained subgraphs. A. J. Goldman.

Effects of a distribution on gap acceptance functions on pedestrian queues. G. H. Weiss.

Gaussian wave functions for polyatomic molecules: integral formulas. M. Krauss. (See above abstracts.)

J. Res. NBS 68C (Eng. and Instr.), No. 1 (Jan.-Mar. 1964)

75 cents.

A Fabry-Perot spectrometer for high-resolution spectroscopy and laser work. K. D. Mielenz, R. B. Stephens, and K. F. Nefflen.

Variable impedance power meter, and adjustable reflection coefficient standard. G. F. Engen.

A laser with a multihole diaphragm. T. Morokuma.

Principles of cryometric impurity determination as applied to samples of small sizes. C. P. Saylor and G. S. Ross.

Reference tables for thermocouples of iridium-rhodium alloys versus iridium. G. F. Blackburn and F. R. Caldwell.

Radio Sci. J. Res. NBS/USNC-URSI, Vol. 68D, No. 3 (Mar.

1964). \$1.00.

An interpretation of rapid changes in the phase of horizontally polarized VLF waves recorded at night over a short path in the southwestern United States. E. E. Gossard and M. R. Paulson.

Precise phase and amplitude measurements on VLF signals propagated through the Arctic zone. F. H. Reder, C. J. Abom, and G. M. R. Winkler.

On the long term phase stability of the $19.8 \mathrm{kc} / \mathrm{s}$ signal trans. mitted from Hawaii, and received at Boulder, Colorado. A. H. Brady.

Oblique propagation of groundwaves across a coastline. Part III. J. R. Wait.

Impedance of a monopole antenna with a radial-wire ground system on an imperfectly conducting half space, Part III. S. W. Maley and R. J. King:

A simplified theory of diffraction at an interface separating two dielectries. J. Kane and S. N. Karp. (See above abstract.)

Variational solution for the admittance of long cylindrical antenna. R. A. Hurd.

Admittance of annular slot antennas radiating into a plasma layer. J. Galejs.

Propagation of plasma waves in a "spoke-wheel" magnetic field. R. L. Liboff. (See above abstract.)

An experimental investigation of signal strength in the area around a transmitter's antipode. R. M. Pipp and J. B. Webster.

Relationship between simultaneous geomagnetic and ionospheric oscillations. H. Rishbeth and O.K. Garriott.

Bibliography on ignition and spark-ignition systems, G. F. Blackburn, NBS Misc. Publ. 251 (Nov. 22, 1963), 15 cents. (Supersedes NBS Circular 580.)

Recommended unit prefixes; Defined values and conversion factors; General physical constants, NBS Misc. Publ, 25. (Oct. 1963), 5 cents (wallet-size plastic card)

Lunar occultations of two discrete radio sources in 1963-1964, J. A. Eddy, NBS Tech. Note 184 (Oct. 11, 1963), 20 cents.
Expanded vinyl fabrics for apparel use, CS 258-63 (Sept. 19, 1963), 10 cents.

Steel spirals for reinforced concrete columns, SPR 53-63, (Oct. 1, 1963), 10 cents.

Determination of differential X-ray photon flux and total beam energy, H. W. Koch and J. S. Pruit, Ed. L. C. Yugan and C. Wu, Nuclear Physics, 5, ch. 2.8.2, 508-553 (1963).

Metallurgical microanalysis with the electron probe, J. R. Cuthill, L. L. Wyman, and H. Yakowitz, J. Metals AIME 15, No. 10, 763-768 (Oct. 1963).

Large longitudinal retarded elastic deformation of rubberlike network polymers. II. Application of a general formulation of nonlinear response, H. Leaderman, F. L. McCrackin, and O. Nakada, Trans. Soc. Rheology VII, 111-123 (1963).

The error rate in a multiple-frequency shift system and the output signal/noise ratio in a frequency modulation and a pulse-code-modulation/freauency-shift system, H. Akima, Intern. Conf. Satellite Communication, London, Nov. 22-28, 1963, pp. 305-309 (Programme and Conference Digest, London, England, 1963.)

Synoptic variations and vertical profiles of large-scale ionospheric irregularities, R. G. Merrill, R. S. Lawrence, and N. J. Roper, J. Geophys. Res. 68, No. 19, 5453-5459 (Oct. 1, 1963)

Excitation of the red and green coronal lines, C. Pecker and R. N. Thomas, Ann. Astrophys. 25, No. 2, 100-108 (1962). Normal congruence subgrouns of the $t \times t$ modular group, M. Newman, Bull. Am. Math. Soc. Res. Announcement 69, No. 5, 719-720 (Sept. 1963).

Radiation impedance of a source near reflectors, R. V. Waterhouse, J. Acoust Soc. Am. 35, No. 8, 1144-1151 (Aug. 1963).

The energy environment in which we live, D. M. Gates, Am. Scientist 51, No. 3, 327-348 (Sept. 1963).

Evidence for field-aligned ionization irregularities between 400 to $100 \mathrm{~km}$ above the earth's surface, T. E. VanZandt, W. Calvert, R. W. Knecht and G. B. Goe, Third Intern. Space Sci. Symp. Proc., Wash., D.C., May 2-8, 1962, pp. 271-273 (John Wiley \& Sons Inc., New York, N.Y., 1962).

Studies in the geomagnetic micropulsation frequency range. W. H. Campbell, IEEE Intern. Conv. Record 11, Pt. 9, 25-28 (Mar. 25-28, 1963).

Determination of the electronic energy levels of molecules by low energy electron impact spectroscopy, J. A. Simpson and S. R. Mielczarek, J. Chem. Phys. Letter 39, 1606 (1963).

Experimental evaluation of a thallium beam frequency standard, R. E. Beehler and D. J. Glaze, Proc. 17th Annual Frequency Control Symp. (1963).

Gas-phase radiolysis of $n$-butane, R. P. Borkowski and P. J. Ausloos, J. Chem. Phys. 39, No. 3, 818-824 (Aug. 1, 1963).

Temperature control of the structure and variations of the quiet ionosphere, J. W. Wright, Proc. Intern. Conf. Ionosphere, July 1962, pp. 19-25 (Institute of Physics and the Physical Society, London, England, 1963).

Some factors affecting the creep behavior of an age-hardenable alloy, W. D. Jenkins and W. A. Willard, Trans. Quart. ASM 56, No. 3, 427-441 (Sept. 1963).

A note on contingency tables involving zero frequencies and the 2I test, H. H. Ku, Technometrics 5, No. 3, 398-400 (Aug. 1963)

Wilmer Souder, pioneer in dental research, G. C. Paffenbarger, N. Y. J. Dentistry 33, No. 5, 191-192 (May 1963).

Equatorial electron density profiles to 5000 KM, Using the incoherent scatter technique, K. L. Bowls, Third Intern Space Sci. Symp. Proc. Washington, D.C., May 2-8, 1962, pp. 253-264 (John Wiley \& Sons, Inc., New York, N.Y., $1963)$.

Physical measurements and experiment design, W. J. Youden, Scuola Azione No. 10, 181-200 (Scuola de Studi Sugli Idrocarburi, San Donato Milanese, Italy, 1961-1962).

Strain release in complete dentures, J. B. Woelfel, G. C. Paffenbarger, and W. T. Sweeney, J. Am. Dental Assoc. 6\%, No. 2, 321 (Aug. 1963).

Questions of general background and methodology relating to aerodynamic phenomena in stellar atmosphere, J. C. Pecker and R. N. Thomas, Il Nuovo Cimento Suppl. 22, No. X, Pt. I, 1-43 (1961).

Observation on reference electrodes for fused-salt systems, M. A. Reid and J. A. Lloyd, Rev. Sci. Instr. 34, No. 8 , 940-941 (Aug. 1963). 
Primary processes in the photolysis of ethyl nitrate, R. E. Rebbert, J. Phys. Chem. 67, No. 9, 1923-1925 (Sept. 1963). High-field liquid $\mathrm{H}_{2}$-cooled aluminum-wound magnet, J. R. Purcell and E. G. Payne, Rev. Sci Instr. 34, 893-897 (Aug. 1963).

Complicating factors in the gas phase photolysis of azomethane, R. E. Rebbert and P. J. Ausloos, J. Phys. Chem. 6r, No. 9, 1925-1928 (Sept. 1963).

Use of transistors in Van de Graaff source leak controls, A. C. B. Richardson and L. Costrell, Nuclear Instr. Methods Letter to Editor 24, No. 1, 129-130 (July 1963).

The formulation of F.D.I. specifications, G. C. Paffenbarger, Intern. Dental J. 13, No. 1, 133-134 (Mar. 1963).

The $F$-region seasonal anomaly, J. W. Wright, J. Geophys. Res. 68, No. 14, 4379-4381 (July 15, 1963).

Effect of hydrostatic pressures on the crvstallization kinetics of natural rubber, G. M. Martin and L. Mandelkern, J. Appl. Phys. 34, No. 8, 2312-2317 (Aug. 1963).

The redefinition of the second and the velocity of light, G. E. Hudson and W. Atkinson, Phys. Today 16, No. 5, 30-32, 34, 36 (May 1963).

The shifts and widths of some stark broadened oxygen lines in an are plasma, W. L. Wiese and P. W. Murphy, Phys. Rev. 131, 2108-2115 (Sept. 1, 1963).

The ionization constants of $o$-nitrophenol and 4-nitro-mcresol from 5 to $60^{\circ}$, R. A. Robinson and A. Peiperl, J. Phys. Chem. 67, 1723 (1963).

Recent developments in neutron source standardization, R. S. Caswell, R. H. Noyce, S. B. Garfinkel, and E. R. Mosburg, Jr., (Proc. Symp. Neutron Detection, Dosimetry and Standardization, International Atomic Energy Ageney, Atomic Energy Research Establishment, Harwell England, Dec. 10-14, 1962), Book, Neutron Dosimetry II, 547-555 (International Atomic Energy Agency, Vienna, 1963).

The following papers were published in Proc. Mountain States Navy Research and Development Clinic, Raton, N. M., Sept. 28-29, 1961, (Raton, Communications and Electronics Foundation, 1963).

Recent progress in cryogenic engineering, R. B. Scott, pp. D1-D4.

Remarks presented at panel discussion on modulation, J. L. Auterman, pp. A131-A132.

Predicting the performance of tropospheric communication links singly and in tandem, A. P. Barsis, K. A. Norton, and P. L. Rice, pp. A71-A104.

Required signal-to-noise ratios, carrier power, and bandwidth to achieve a given performance for multichannel radio communication systems, E. R. Florman and J. J. Tary, pp. A $135-\mathrm{A} 140$.

VLF propagation, W. L. Taylor and A. G. Jean, pp. A45-A66. Millimeter wave research at the National Bureau of Standards, R. W. Zimmerer, pp. A109-A112.

Some low temperature properties of materials, R. H. Kropschot, pp. 1)5-D6.

Aspects of radio meteorology, J. D. Horn, pp. C51-C56.

Problems and prospects in electrical properties of materials, J. L. Dalke, pp. D7-D14.

Noise in VLF to HF bands and prediction of communication reliability, W. Q. Crichlow, pp. A67-A70.

Signal-to-noise characteristics of some typical systems and their comparisons, H. Akima, pp. A125-A130.

Cyclotron resonances in slightly ionized gases, F. Fehsenfeld, J. Chem. Phys. 39, No. 7, 1653-1661 (Oct. 1, 1963).

Some physical properties of organic denture base materials, J. B. Woelfel, G. C. Paffenbarger, and W. T. Sweeney, J. Am. Dental Ássoc. 6\%, 489-504 (Oct. 1963).

Dependence of power output of a gas laser on the length and rate of excitation of the discharge, J. A. White, Appl. Phys. Letters 3, No. 7, 107-109 (Oct. 1, 1963).

Shifts and widths of some stark-broadened oxygen lines in an are plasma, W. L. Wiese and P. W. Murphy, Phys. Rev. 131, No. 5, 2108-2115 (Sept. 1963).

The adsorption of methane and nitrogen on silica gel, synthetic zeolite and charcoal, A. J. Kidnay and J. M. Hiza, J Phys. Chem. 67, 1725-1727 (1963).

Studying the lunar tidal variations in the $D$ region of the ionosphere by means of very-low-frequency phase observa- tions, A. H. Brady and D. D. Crombie, J. Geophys. Res. 68, No. 19, 5437-5442 (Oct. 1, 1963).

Natural electromagnetic field fluctuations in the 3.0 to 0.02 cps range, W. H. Campbell, Proc. IEEE 51, 1337-1342 (Oct. 1963).

Current development in an electronically scanned antenna, H. V. Cottony, Proc. Symp. Electromagnetic Theory and Antennas, Copenhagen, June 25-30, 1962, pp. 1289-1294 (Pergamon Press, Oxford, London, 1963).

Airglow research, F. E. Roach, Trans. Am. Geophys. Union 44, No. 2, 431-432 (June 1963).

Velocity-depth relationship in microelectrophoresis cell for asphaltenes in nitromethane, J. R. Wright and R. R. Minesinger, J. Colloid Sci. 18, 802-804 (Oct. 1963).

Some results of a new method for obtaining ionospheric $\mathrm{N}(\mathrm{h})$ profiles and their bearing on the structure of the lower $F$ region, A. K. Paul and J. W. Wright, J. Geophys. Res. 68, No. 19, 5413-5420 (Oct. 1, 1963).

A 100 megapulse per second binary counter with impedance steering, S. B. Geller and P. A. Mantek, IEEE Trans. Electronic Computers EC-12, No. 5, 568 (Oct. 1963).

Mathematics and experimental science, W. J. Youden, Sci. Teacher 30, No. 5, 23-26 (Sept. 1963).

Line shapes, saturation behavior, and temperature studies in the nuclear resonance of nickel, R. L. Streever and L. H. Bennett, Phys. Rev. 131, No. 5, 2000-2009 (Sept. 1, 1963).

Impedance of Leclanche cells and batteries, R. J. Brodd and H. J. DeWane, J. Electrochem. Soc. 110, No. 11, 1091-1097 (Nov. 1963)

Hyperfine structure and isotope shifts in the 2537-A line of mercury by a new interferometric method, W. G. Schweitzer, Jr., J. Opt. Soc. Am. 53, No. 9, 1055-1072 (Sept. 1963).

Absolute scale of oscillator strenghts, C. W. Allen and C. H. Coriiss, Monthly Notices Roy. Astron. Soc. 126, No. 1, $37-40$ (1963).

Stable $6300 \mathrm{~A}$ auroral ares in mid-latitudes, F. E. Roach and J. R. Roach, Planet. Space Sci. 11, 523-545 (1963).

Relation of partial (110) pole figures to thickness and microstructure of electrodeposited copper, F. Ogburn and C. J. Newton, J. Electrochem. Soc. 110, No. 11, 1148-1150 (Nov. 1963).

Regulation of ionization gauge emission current to better than $0.05 \%, K$. W. Yee and R. J. Carpenter, Rev. Sci. Instr 34, No. 10, 1101-1103 (Oct. 1963).

Errors in "drop" calorimetry due to sample container transitions, D. C. Ginnings, J. Phys. Chem. 67, 1917-1918 (1963).

A theory of incoherent scattering of radio waves by a plasma. III Scattering in a partially ionized gas, J. P. Dougherty and D. T. Farley, Jr., J. Geophys. Res. 68, 5473-5486 (Oct. 1, 1963)

Energy dependence of proportional-counter fast-neutron dosimeters, R. S. Caswell, W. B. Beverly and V. Speigel, Jr., (Proc. Symp. Neutron Detection, Dosimetry and Standardization, Atomic Energy, Atomic Energy Research Establishment, Harwell, England, Dec. 10-14, 1962), Book, Neutron Dosimetry II, 227-237 (July 1963).

Projection X-ray microscopy of roofing materials, S. B. Newman and S. H. Greenfeld, Mater. Res. Std. 3, No. 11, 893-898 (Nov. 1963).

Normal congruence subgroups of the $t \times t$ modular group, M. Newman, Bull. Am. Math. Soc. 69, No. 5, 719-720 (Sept. 1963).

Growth and evaporation kinetics and surface diffusion of $\mathrm{K}$ and $\mathrm{Hg}$ crystal whiskers, R. L. Parker, R. L. Anderson, and S. C. Hardy, Applied Phys. Letters 3, No. 6, 93-95 (Sept. 15, 1963).

Very-low-frequency phase observations of solar flare ionization in the $D$ region of the ionosphere, C. J. Chilton, F. K. Steele, and R. B. Norton, J. Geophys. Res. 68, No. 19, 5421-5435 (Oct. 1, 1963).

Generalized variational principles for electromagnetic vibrations: application to the theory of waveguide junctions, D. M. Kerns, Proc. Symp. Electromagnetic Theory and Antennas, Copenhagen, June 25-30, 1962, pp. 253-259 (Pergamon Press, Oxford, London, 1963).

The following papers were published in Advances in Cryogenic Engineering, Volume 8 (1963). 
Some idealized solutions for choking, two-phase flow of hydrogen, nitrogen, and oxygen, R. V. Smith, Paper J-10, pp. 563-573.

$P-\rho-T$ values for neon from $27^{\circ}$ to $300^{\circ} \mathrm{K}$ for pressures to $200 \mathrm{~atm}$ using corresponding states theory, R. D. McCarty, R. B. Stewart, and K. D. Timmerhaus, Paper C-3, pp. $135-145$.

An apparatus to determine the solid-vapor equilibria of binary cryogenic systems, M. J. Hiza and R. N. Herring, Paper C-5, pp. 158-161.

A comparison of the low-temperature adsorption of nitrogen and methane from hydrogen gas on three different adsorbents, M. J. Hiza and A. J. Kidnay, Paper C-7, pp. 174-182.

A refrigeration system incorporating a low-capacity, highspeed, gas-bearing-supported expansion turbine, D. B. Mann, H. Sixsmith, W. A. Wilson, and B. W. Birmingham, Paper D-5, pp. 221-227.

Experimental determination of the bulk density of boiling liquid oxygen, R. W. Arnett, D. R. Millhiser, and W. H. Probert, Paper E-4, pp. 256-262.

Thermal conductivity of solid $\mathrm{H}_{2} \mathrm{O}$ and $\mathrm{D}_{0} \mathrm{O}$ at low temperatures, J. W. Dean and K. D. Timmerhaus, Paper E-5, pp. 263-267.

Linear thermal expansion of elastomers in the range $300^{\circ}$ to $76^{\circ} \mathrm{K}, \mathrm{R}$. F. Robbins, Y. Ohori, and D. H. Weitzel, Paper E-8, pp. 287-299 (1963).
Solid formation in flowing cryogenic fluids, D. B. Chelton, B. W. Birmingham, and J. W. Dean, Paper E-11, pp. 311-314.

Temperature measurements in cryogenic engineering, R. J. Corruccini, Paper F-1, pp. 315-333.

Pressure measurements in cryogenic systems, P. Smelser, Paper F-7, pp. 378-386.

Perlite for cryogenic insulation, R. H. Kropschot and R. W. Burgess, Paper G-7, pp. 425-436.

Force and seal evaluation of elastomeric O-rings, P. R. Ludtke and D. H. Weitzel, Paper H-6, pp. 467-477.

Experimental investigation of liquid-hydrogen cooling by helium gas injection, A. F. Schmidt, Paper J-5, pp. 521-528.

Liquid requirements for the cool-down of cryogenic equipment, R. B. Jacobs, Paper J-6, pp. 529-535.

Cool-down and warm-up of large powder-insulated dewars, F. Kreith, J. W. Dean, and L. Brooks, Paper J-7, pp. $536-543$.

*Publications for which a price is indicated are available by purchase from the Superintendent of Documents, Government Printing Office, Washington, D.C. 20402 (foreign postage, one-fourth additional). Reprints from outside journals and the NBS Journal of Research may often be obtained directly from the authors. 